\title{
Total Synthesis of (-)-Rhodomollanol A
}

Jianhong Gao, ${ }^{\dagger}$ Peirong Rao,${ }^{\dagger}$ Kaixiang Xu, ${ }^{\dagger}$ Shuaifeng Wang,${ }^{\dagger}$ Yufei Wu,${ }^{\dagger}$ Chi He,${ }^{\dagger}$ and Hanfeng Ding*,,+

tDepartment of Chemistry, Zhejiang University, Hangzhou 310058, China

${ }^{t}$ Key Laboratory of Organosilicon Chemistry and Material Technology of Ministry of Education, Hangzhou Normal University, Hangzhou 311121, China

E-mail : hfding@zju.edu.cn

\section{Supporting Information Available}

I) Experimental Procedures and Spectroscopic Data of Compounds

II) Abbreviations

III) References

IV) ${ }^{1} \mathrm{H}$ and ${ }^{13} \mathrm{C}$ NMR Spectra of Compounds

V) HPLC Chromatographs 


\section{I) Experimental Procedures and Spectroscopic Data of Compounds}

General Procedures. All reactions were carried out under an argon atmosphere with dry solvents under anhydrous conditions, unless otherwise noted. Anhydrous methylene chloride $\left(\mathrm{CH}_{2} \mathrm{Cl}_{2}\right)$ and hexamethyl phosphoryl triamide (HMPA) were distilled before use from calcium hydride. Anhydrous tetrahydrofuran (THF) and toluene were distilled before use from sodium-benzophenone ketyl. Acetic acid $(\mathrm{AcOH})$, acetone, 1,2-dichlorobenzene (o-DCB), dimethyl sulfoxide (DMSO), ethanol (EtOH), ethyl acetate (EtOAc), 1,1,1,3,3,3-hexafluoro-2-propanol (HFIP), isopropanol $(i-\mathrm{PrOH})$, methanol $(\mathrm{MeOH})$, triethylamine $\left(\mathrm{Et}_{3} \mathrm{~N}\right)$ and pyridine were purchased at the highest commercial quality and used without further purification. Reactions that required heating were operated on a magnetic stirrer with an oil bath. Solvent degassing was conducted by bubbling with a stream of argon for 15-20 min. Reactions were monitored by thin-layer chromatography (TLC) carried out on $0.25 \mathrm{~mm}$ E. Merck silica gel plates (60F-254) using UV light as visualizing agent and an ethanolic solution of ammonium molybdate, anisaldehyde, and heat as developing agents. E. Merck silica gel (60, particle size $0.040-0.063 \mathrm{~mm})$ was used for flash column chromatography. Yields refer to chromatographically homogeneous materials. NMR spectra were recorded on a Bruker AV-400 or Agilent DD2-600 and calibrated using residual undeuterated solvent as an internal reference. The following abbreviations were used to explain the multiplicities: $\mathrm{s}=$ singlet, $\mathrm{d}=$ doublet, $\mathrm{t}=$ triplet, $\mathrm{q}=$ quartet, $\mathrm{br}=$ broad, $\mathrm{dd}=$ doublet of doublets, $\mathrm{ddd}=$ doublet of doublet of doublets, $\mathrm{td}=$ triplet of doublets, $\mathrm{m}=$ multiplet. Melting points (m.p.) are uncorrected, and recorded on a Buchi B-540 
melting point apparatus. Optical rotation data were obtained on a PerkinElmer Model 341 Polarimeter. High performance liquid chromatography (HPLC) analyses were performed on a Shimadzu LC-20A HPLC system. Detection of eluent was carried out with a photodiode array detector at $210 \mathrm{~nm}$. High-resolution mass spectra (HRMS) were recorded on a Waters MALDI SYNAPT G2-Si High Definition Mass Spectrometry.

\section{Procedures for the Preparation of Aldehyde $15^{1}$}<smiles>CC1=C2CCCC3(OCCO3)C2(C)CCC1=O</smiles>

$16,>95 \%$ ee

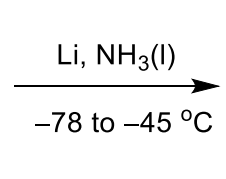

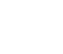$$
\text { (cHO }
$$

15

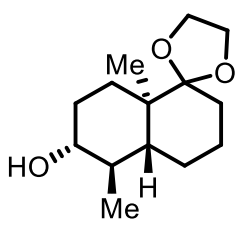

S-1

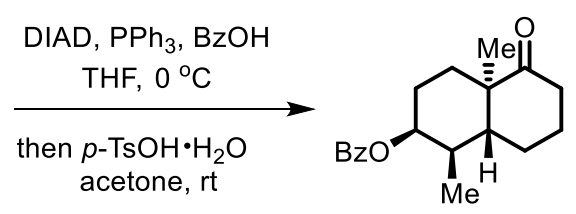

$72 \%$ (2 steps)
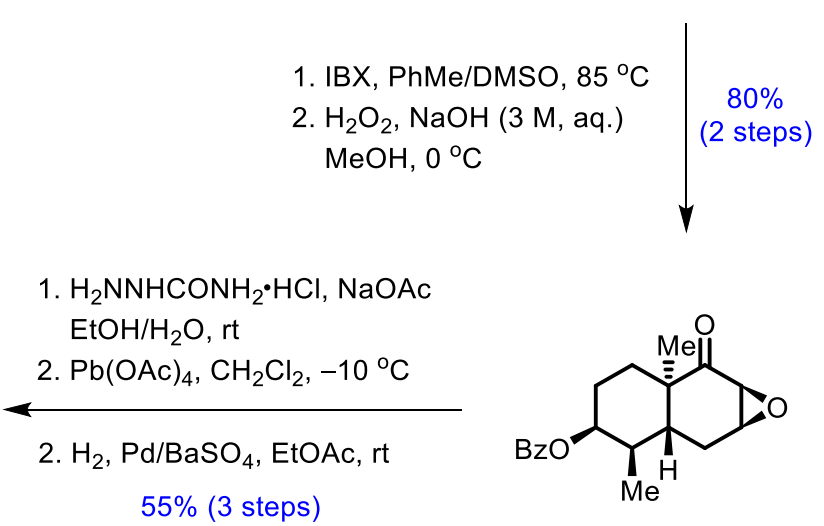

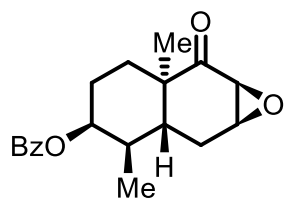

S-3

Scheme S1. Preparation of aldehyde 15

\section{Preparation of bicyclic ketone S-2:}

To a stirred solution of liquid ammonia $(400 \mathrm{~mL})$ at $-78^{\circ} \mathrm{C}$ was added $\mathrm{Li}(9.44 \mathrm{~g}$, 1.34 mol, 4.0 equiv). The resulting mixture was stirred at $-78^{\circ} \mathrm{C}$ for $30 \mathrm{~min}$ before a solution of enone $\mathbf{1 6}^{2}$ (>95\% ee, $80.0 \mathrm{~g}, 339 \mathrm{mmol}, 1.0$ equiv) in THF (200 mL) was added. The resulting mixture was stirred at $-45^{\circ} \mathrm{C}$ for $4 \mathrm{~h}$ before quenched with EtOH $(80 \mathrm{~mL})$. The ammonia was evaporated under a stream of nitrogen at $30{ }^{\circ} \mathrm{C}$, and the residue was partitioned between EtOAc (200 mL) and $\mathrm{NH}_{4} \mathrm{Cl}(200 \mathrm{~mL}$, sat. aq.). The 
layers were separated, and the aqueous layer was extracted with EtOAc $(3 \times 200 \mathrm{~mL})$. The combined organic layers were dried $\left(\mathrm{Na}_{2} \mathrm{SO}_{4}\right)$ and concentrated in vacuo to afford the crude alcohol S-1 as a yellow oil, which was used directly without further purification.

To a stirred solution of alcohol S-1 (crude, obtained above) in THF (250 $\mathrm{mL}$ ) at 0 ${ }^{\circ} \mathrm{C}$ were sequentially added $\mathrm{BzOH}\left(48.8 \mathrm{~g}, 400\right.$ mmol, 1.2 equiv), $\mathrm{PPh}_{3}$ (105 g, 400 mmol, 1.2 equiv) and DIAD (78.7 mL, $398 \mathrm{mmol}, 1.2$ equiv). The resulting mixture was stirred at $0{ }^{\circ} \mathrm{C}$ for $1 \mathrm{~h}$ before a solution of $p$ - $\mathrm{TsOH} \cdot \mathrm{H}_{2} \mathrm{O}(76.0 \mathrm{~g}, 400 \mathrm{mmol}, 1.2$ equiv) in acetone $(300 \mathrm{~mL})$ was added. The resulting mixture was stirred at room temperature for $2 \mathrm{~h}$ before diluted with EtOAc $(200 \mathrm{~mL})$ and quenched with $\mathrm{NaHCO}_{3}$ (500 mL, sat. aq.). The layers were separated, and the aqueous layer was extracted with EtOAc $(3 \times 200 \mathrm{~mL})$. The combined organic layers were dried $\left(\mathrm{Na}_{2} \mathrm{SO}_{4}\right)$ and concentrated in vacuo. Flash column chromatography (silica gel, hexanes:EtOAc 20:1) afforded bicyclic ketone S-2 (91.5 g, 72\% over two steps) as a white solid. S-2: m.p. 92-94 ${ }^{\circ} \mathrm{C}$ (hexanes/EtOAc); $R_{\mathrm{f}}=0.50$ (silica gel, hexanes:EtOAc 4:1); $97 \%$ ee [Daical Chiralpak AD-H $(0.46 \mathrm{~cm} \times 25 \mathrm{~cm}), n$-hexane/2-propanol $=97 / 3, \mathrm{v}=1.0 \mathrm{~mL} \cdot \mathrm{min}^{-1}, \lambda$ $=210 \mathrm{~nm}, \mathrm{t}($ major $)=10.80 \mathrm{~min}, \mathrm{t}($ minor $)=13.88 \mathrm{~min}] ;[\alpha]_{\mathrm{D}}^{20}=+3.2\left(\mathrm{c}=0.5, \mathrm{CHCl}_{3}\right)$; ${ }^{1} \mathrm{H}$ NMR $\left(400 \mathrm{MHz}, \mathrm{CDCl}_{3}\right) \delta=8.05-7.92(\mathrm{~m}, 2 \mathrm{H}), 7.52(\mathrm{t}, J=7.4 \mathrm{~Hz}, 1 \mathrm{H}), 7.41(\mathrm{t}$, $J=7.6 \mathrm{~Hz}, 2 \mathrm{H}), 5.17(\mathrm{~d}, J=2.4 \mathrm{~Hz}, 1 \mathrm{H}), 2.70-2.60(\mathrm{~m}, 1 \mathrm{H}), 2.24(\mathrm{dd}, J=14.4,4.3$ Hz, $1 \mathrm{H}), 2.11-2.04(\mathrm{~m}, 1 \mathrm{H}), 1.97-1.61(\mathrm{~m}, 7 \mathrm{H}), 1.58-1.52$ (m, $1 \mathrm{H}), 1.50-1.40$ (m, 1 $\mathrm{H}), 1.16(\mathrm{~s}, 3 \mathrm{H}), 0.93 \mathrm{ppm}(\mathrm{d}, J=6.6 \mathrm{~Hz}, 3 \mathrm{H}) ;{ }^{13} \mathrm{C} \mathrm{NMR}\left(100 \mathrm{MHz}, \mathrm{CDCl}_{3}\right) \delta=215.7$, 166.0, 132.9, 130.7, $129.5(2 \mathrm{C}), 128.4(2 \mathrm{C}), 74.2,48.5,45.5,37.2,34.4,27.3,26.0(2 \mathrm{C})$ 
23.1, 16.2, 16.1 ppm; HRMS (ESI): calcd for $\mathrm{C}_{19} \mathrm{H}_{24} \mathrm{O}_{3} \mathrm{Na}^{+}[\mathrm{M}+\mathrm{Na}]^{+} 323.1618$, found 323.1623.

\section{Preparation of epoxide S-3:}

To a stirred solution of bicyclic ketone $\mathbf{S - 2}(99.0 \mathrm{~g}, 330 \mathrm{mmol}, 1.0$ equiv) in DMSO/toluene $(3: 1,1 \mathrm{~L})$ at $85{ }^{\circ} \mathrm{C}$ was added IBX (185 g, $661 \mathrm{mmol}, 2.0$ equiv). The resulting mixture was stirred at $85^{\circ} \mathrm{C}$ for $12 \mathrm{~h}$ before another portion of IBX ( $185 \mathrm{~g}$, $661 \mathrm{mmol}, 2.0$ equiv) was added. The reaction was stirred at $85{ }^{\circ} \mathrm{C}$ for an additional 12 h before cooled to $0{ }^{\circ} \mathrm{C}$, diluted with EtOAc $(1000 \mathrm{~mL})$ and quenched with $\mathrm{Na}_{2} \mathrm{~S}_{2} \mathrm{O}_{3}$ (500 mL, sat. aq.). The layers were separated, and the aqueous layer was extracted with EtOAc $(3 \times 200 \mathrm{~mL})$. The combined organic layers were dried $\left(\mathrm{Na}_{2} \mathrm{SO}_{4}\right)$ and concentrated in vacuo to afford the crude enone as a white solid, which was used directly without further purification.

To a stirred solution of enone (crude, obtained above) in $\mathrm{MeOH}(1000 \mathrm{~mL})$ at $0{ }^{\circ} \mathrm{C}$ were sequentially added $\mathrm{H}_{2} \mathrm{O}_{2}(30 \%$ wt/wt aq., $84.8 \mathrm{~mL}, 838 \mathrm{mmol}, 2.5$ equiv) and $\mathrm{NaOH}$ (3.0 M aq., $167 \mathrm{~mL}, 495 \mathrm{mmol}, 1.5$ equiv). The resulting mixture was stirred at $0{ }^{\circ} \mathrm{C}$ for 30 min before diluted with $\mathrm{CH}_{2} \mathrm{Cl}_{2}(500 \mathrm{~mL})$ and quenched with $\mathrm{NH}_{4} \mathrm{Cl}(500$ $\mathrm{mL}$, sat. aq.). The layers were separated, and the aqueous layer was extracted with $\mathrm{CH}_{2} \mathrm{Cl}_{2}(3 \times 200 \mathrm{~mL})$. The combined organic layers were dried $\left(\mathrm{Na}_{2} \mathrm{SO}_{4}\right)$ and concentrated in vacuo. Flash column chromatography (silica gel, hexanes:EtOAc 6:1) afforded epoxide $\mathbf{S - 3}$ (82.9 g, 80\% over two steps) as a white solid. S-3: m.p. 101-103 ${ }^{\circ} \mathrm{C}$ (hexanes/EtOAc); $R_{\mathrm{f}}=0.50$ (silica gel, hexanes:EtOAc $\left.4: 1\right) ;[\alpha]_{\mathrm{D}}{ }^{20}=-3.0(\mathrm{c}=0.5$, $\left.\mathrm{CHCl}_{3}\right) ;{ }^{1} \mathrm{H} \mathrm{NMR}\left(400 \mathrm{MHz}, \mathrm{CDCl}_{3}\right) \delta=8.01(\mathrm{dd}, J=8.0,1.4 \mathrm{~Hz}, 2 \mathrm{H}), 7.56(\mathrm{t}, J=$ 
$7.4 \mathrm{~Hz}, 1 \mathrm{H}), 7.45$ (t, $J=7.6 \mathrm{~Hz}, 2 \mathrm{H}), 5.19(\mathrm{q}, J=2.5 \mathrm{~Hz}, 1 \mathrm{H}), 3.57$ (t, $J=2.4 \mathrm{~Hz}, 1$ H), $3.25(\mathrm{~d}, J=3.4 \mathrm{~Hz}, 1 \mathrm{H}), 2.44(\mathrm{~s}, 1 \mathrm{H}), 2.16(\mathrm{~d}, J=4.2 \mathrm{~Hz}, 1 \mathrm{H}), 2.05-1.96(\mathrm{~m}, 1$ H), 1.87-1.78 (m, $2 \mathrm{H}), 1.72(\mathrm{~d}, J=13.8 \mathrm{~Hz}, 2 \mathrm{H}), 1.65-1.60(\mathrm{~m}, 1 \mathrm{H}), 1.05(\mathrm{~s}, 3 \mathrm{H})$, $0.98 \mathrm{ppm}(\mathrm{d}, J=6.7 \mathrm{~Hz}, 3 \mathrm{H}) ;{ }^{13} \mathrm{C} \mathrm{NMR}\left(100 \mathrm{MHz}, \mathrm{CDCl}_{3}\right) \delta=206.9,166.2,133.1$, $130.5,129.7$ (2C), $128.6(2 \mathrm{C}), 73.7,52.8,51.9,45.6,34.2,32.1,26.7,25.8,24.3,15.8$, 15.5 ppm; HRMS (ESI): calcd for $\mathrm{C}_{19} \mathrm{H}_{22} \mathrm{O}_{4} \mathrm{Na}^{+}[\mathrm{M}+\mathrm{Na}]^{+}$337.1410, found 337.1416.

\section{Preparation of aldehyde 15:}

To a stirred solution of epoxide $\mathbf{S - 3}$ (78.5 g, $250 \mathrm{mmol}$, 1.0 equiv) in $\mathrm{EtOH} / \mathrm{H}_{2} \mathrm{O}$ $(2.5: 1,700 \mathrm{~mL})$ at room temperature were sequentially added $\mathrm{NaOAc}(23.8 \mathrm{~g}, 290$ mmol, 1.2 equiv) and $\mathrm{H}_{2} \mathrm{NNHCONH}_{2} \bullet \mathrm{HCl}(65.7 \mathrm{~g}, 587$ mmol, 2.3 equiv). The resulting mixture was stirred at room temperature for $1 \mathrm{~h}$ before diluted with $\mathrm{CH}_{2} \mathrm{Cl}_{2}(200 \mathrm{~mL})$ and quenched with brine (200 $\mathrm{mL}$, sat. aq.). The layers were separated, and the aqueous layer was extracted with $\mathrm{CH}_{2} \mathrm{Cl}_{2}(3 \times 200 \mathrm{~mL})$. The combined organic layers were dried $\left(\mathrm{Na}_{2} \mathrm{SO}_{4}\right)$ and concentrated in vacuo to afford the crude hydrazone as a yellow oil, which was used directly without further purification.

To a stirred solution of hydrazone (crude, obtained above) in $\mathrm{CH}_{2} \mathrm{Cl}_{2}(400 \mathrm{~mL})$ at $-10{ }^{\circ} \mathrm{C}$ was added $\mathrm{Pb}(\mathrm{OAc})_{4}(129 \mathrm{~g}, 291 \mathrm{mmol}, 1.2$ equiv). The resulting mixture was stirred at $-10{ }^{\circ} \mathrm{C}$ for 30 min before quenched with $\mathrm{H}_{2} \mathrm{O}(200 \mathrm{~mL})$ and filtered through a short pad of celite. The filtrate was extracted with $\mathrm{CH}_{2} \mathrm{Cl}_{2}(3 \times 200 \mathrm{~mL})$, and the combined organic layers were washed with $\mathrm{NaHCO}_{3}\left(200 \mathrm{~mL}\right.$, sat. aq.), dried $\left(\mathrm{Na}_{2} \mathrm{SO}_{4}\right)$ and concentrated in vacuo. Flash column chromatography (silica gel, hexanes:EtOAc 6:1) afforded the corresponding alkyne as a white solid. 
To a stirred solution of alkyne (obtained above) in EtOAc $(120 \mathrm{~mL})$ at room temperature were sequentially added $\mathrm{SiO}_{2}(12.0 \mathrm{~g})$ and $\mathrm{Pd} / \mathrm{BaSO}_{4}(2.2 \mathrm{~g})$. The resulting mixture was stirred at room temperature under hydrogen atmosphere ( $1 \mathrm{~atm})$ for $30 \mathrm{~min}$ before filtered through a short pad of celite. The filtrate was concentrated in vacuo. Flash column chromatography (silica gel, hexanes:EtOAc 6:1) afforded aldehyde 15 (41.3 g, 55\% over three steps) as a white solid. 15: m.p. $117-119{ }^{\circ} \mathrm{C}$ (hexanes/EtOAc); $R_{\mathrm{f}}=0.50$ (silica gel, hexanes:EtOAc 6:1); $[\alpha]_{\mathrm{D}}{ }^{20}=+2.5\left(\mathrm{c}=0.5, \mathrm{CHCl}_{3}\right) ;{ }^{1} \mathrm{H} \mathrm{NMR}(400$ $\left.\mathrm{MHz}, \mathrm{CDCl}_{3}\right) \delta=9.77(\mathrm{~d}, J=2.5 \mathrm{~Hz}, 1 \mathrm{H}), 8.08(\mathrm{~d}, J=7.3 \mathrm{~Hz}, 2 \mathrm{H}), 7.58(\mathrm{t}, J=7.4$ Hz, $1 \mathrm{H}), 7.48(\mathrm{t}, J=7.6 \mathrm{~Hz}, 2 \mathrm{H}), 5.76(\mathrm{dd}, J=17.4,10.9 \mathrm{~Hz}, 1 \mathrm{H}), 5.23-5.18(\mathrm{~m}, 1$ H), 5.08-5.02 (m, $2 \mathrm{H}), 2.45-2.35(\mathrm{~m}, 2 \mathrm{H}), 2.28-2.21(\mathrm{~m}, 1 \mathrm{H}), 2.00-1.92(\mathrm{~m}, 1 \mathrm{H})$, $1.81(\mathrm{dd}, J=14.0,9.2 \mathrm{~Hz}, 3 \mathrm{H}), 1.30-1.22(\mathrm{~m}, 1 \mathrm{H}), 1.02(\mathrm{~s}, 3 \mathrm{H}), 0.88 \mathrm{ppm}(\mathrm{d}, J=6.8$ $\mathrm{Hz}, 3 \mathrm{H}) ;{ }^{13} \mathrm{C} \mathrm{NMR}\left(100 \mathrm{MHz}, \mathrm{CDCl}_{3}\right) \delta=202.5,166.2,148.8,133.1,130.8,129.7$ (2C), 128.6 (2C), 112.9, 74.5, 45.2, 40.6, 39.0, 36.3, 33.8, 26.4, 17.3, 15.5 ppm; HRMS (ESI): calcd for $\mathrm{C}_{19} \mathrm{H}_{24} \mathrm{O}_{3} \mathrm{Na}^{+}[\mathrm{M}+\mathrm{Na}]^{+}$323.1618, found 323.1626.

\section{Preparation of phenyl bromide 17:}
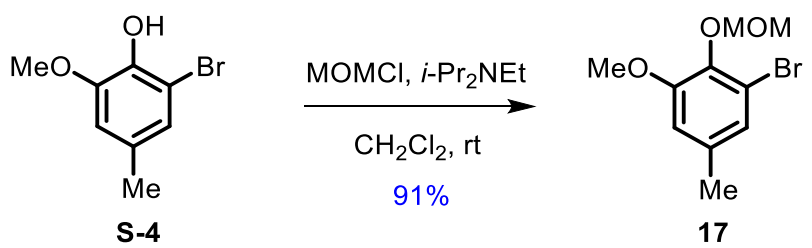

To a stirred solution of bromophenol $\mathbf{S}-4^{3}$ (10.0 g, 46.3 mmol, 1.0 equiv) in $\mathrm{CH}_{2} \mathrm{Cl}_{2}$ $(100 \mathrm{~mL})$ at room temperature were sequentially added $i-\operatorname{Pr}_{2} \mathrm{NEt}(16.1 \mathrm{~mL}, 92.6 \mathrm{mmol}$, 2.0 equiv) and $\mathrm{MOMCl}(4.20 \mathrm{~mL}, 55.6 \mathrm{mmol}, 1.2$ equiv). The resulting mixture was stirred at room temperature for $2 \mathrm{~h}$ before quenched with $\mathrm{NaHCO}_{3}(50 \mathrm{~mL}$, sat. aq.). The layers were separated, and the aqueous layer was extracted with $\mathrm{CH}_{2} \mathrm{Cl}_{2}(3 \times 50$ 
$\mathrm{mL})$. The combined organic layers were dried $\left(\mathrm{Na}_{2} \mathrm{SO}_{4}\right)$ and concentrated in vacuo. Flash column chromatography (silica gel, hexanes:EtOAc 4:1) afforded phenyl bromide 17 (11.0 g, 91\%) as a white solid. 17: m.p. $108-110^{\circ} \mathrm{C}$ (hexanes/EtOAc); $R_{\mathrm{f}}$ $=0.50\left(\right.$ silica gel, hexanes:EtOAc 10:1); ${ }^{1} \mathrm{H}$ NMR $\left(400 \mathrm{MHz}, \mathrm{CDCl}_{3}\right) \delta=6.96(\mathrm{~d}, J=$ $1.0 \mathrm{~Hz}, 1 \mathrm{H}), 6.66(\mathrm{~d}, J=1.2 \mathrm{~Hz}, 1 \mathrm{H}), 5.12(\mathrm{~s}, 2 \mathrm{H}), 3.82(\mathrm{~s}, 3 \mathrm{H}), 3.65(\mathrm{~s}, 3 \mathrm{H}), 2.28$ $\operatorname{ppm}(\mathrm{s}, 3 \mathrm{H}) ;{ }^{13} \mathrm{C} \mathrm{NMR}\left(100 \mathrm{MHz}, \mathrm{CDCl}_{3}\right) \delta=153.1,141.1,135.4,125.3,117.4,112.7$, 98.7, 58.0, 56.1, 21.1 ppm; HRMS (ESI): calcd for $\mathrm{C}_{10} \mathrm{H}_{13} \mathrm{BrO}_{3} \mathrm{Na}^{+}[\mathrm{M}+\mathrm{Na}]^{+}$282.9940, found 282.9946 .

Preparation of alcohols 18 and C7-epi-18:

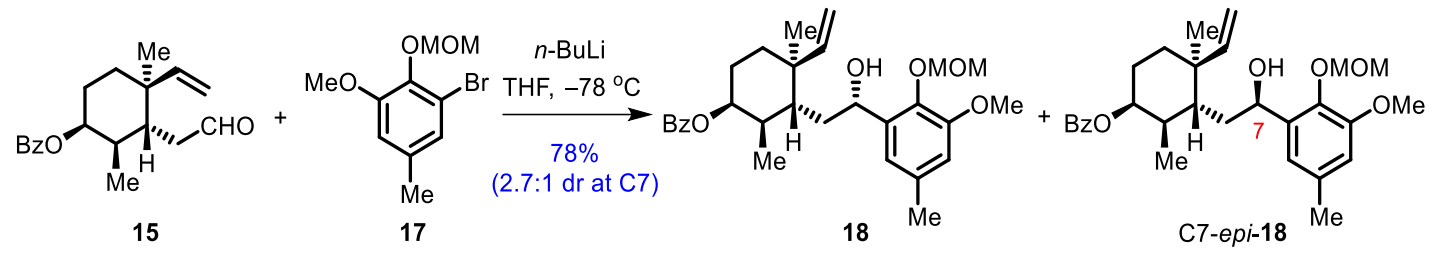

To a stirred solution of phenyl bromide 17 (27.3 g, $105 \mathrm{mmol}, 1.1$ equiv) in THF $(150 \mathrm{~mL})$ at $-78{ }^{\circ} \mathrm{C}$ was added $n$-BuLi $(45.8 \mathrm{~mL}, 2.4 \mathrm{M}$ in hexanes, $110 \mathrm{mmol}, 1.1$ equiv). The resulting mixture was stirred at $-78{ }^{\circ} \mathrm{C}$ for $10 \mathrm{~min}$ before a solution of aldehyde 15 (30.0 g, 100 mmol, 1.0 equiv) in THF (50 mL) was added. The reaction was stirred at $-78{ }^{\circ} \mathrm{C}$ for an additional $1 \mathrm{~h}$ before quenched with $\mathrm{NH}_{4} \mathrm{Cl}(50 \mathrm{~mL}$, sat. aq.). The layers were separated, and the aqueous layer was extracted with EtOAc $(3 \times$ $50 \mathrm{~mL})$. The combined organic layers were dried $\left(\mathrm{Na}_{2} \mathrm{SO}_{4}\right)$ and concentrated in vacuo. Flash column chromatography (silica gel, hexanes:EtOAc 4:1) afforded alcohols $\mathbf{1 8}$ (27.5 g, 57\%) and C7-epi-18 (11.1 g, 21\%) as white solids. 18: m.p. $122-124{ }^{\circ} \mathrm{C}$ (hexanes/EtOAc); $R_{\mathrm{f}}=0.45$ (silica gel, hexanes:EtOAc $\left.2: 1\right) ;[\alpha]_{\mathrm{D}}{ }^{20}=+28.0(\mathrm{c}=0.5$, $\left.\mathrm{CHCl}_{3}\right) ;{ }^{1} \mathrm{H} \mathrm{NMR}\left(400 \mathrm{MHz}, \mathrm{CDCl}_{3}\right) \delta=8.10-8.07(\mathrm{~m}, 2 \mathrm{H}), 7.58(\mathrm{t}, J=7.4 \mathrm{~Hz}, 1 \mathrm{H})$, 
$7.47(\mathrm{t}, J=7.6 \mathrm{~Hz}, 2 \mathrm{H}), 6.72(\mathrm{~s}, 1 \mathrm{H}), 6.64(\mathrm{~s}, 1 \mathrm{H}), 6.01(\mathrm{dd}, J=17.5,10.8 \mathrm{~Hz}, 1 \mathrm{H})$, $5.20(\mathrm{~d}, J=1.4 \mathrm{~Hz}, 1 \mathrm{H}), 5.14-5.04(\mathrm{~m}, 4 \mathrm{H}), 4.93-4.88(\mathrm{~m}, 1 \mathrm{H}), 3.80(\mathrm{~s}, 3 \mathrm{H}), 3.51$ (s, $3 \mathrm{H}), 2.69(\mathrm{~d}, J=6.5 \mathrm{~Hz}, 1 \mathrm{H}), 2.30(\mathrm{~s}, 3 \mathrm{H}), 1.99-1.81(\mathrm{~m}, 6 \mathrm{H}), 1.47-1.41(\mathrm{~m}, 1$ $\mathrm{H}), 1.27-1.20(\mathrm{~m}, 1 \mathrm{H}), 1.07(\mathrm{~d}, J=6.8 \mathrm{~Hz}, 3 \mathrm{H}), 1.02 \mathrm{ppm}(\mathrm{s}, 3 \mathrm{H}) ;{ }^{13} \mathrm{C}$ NMR $(100$ $\left.\mathrm{MHz}, \mathrm{CDCl}_{3}\right) \delta=166.3,151.6,150.2,141.2,138.7,134.5,132.9,131.0,129.7$ (2C), $128.5(2 \mathrm{C}), 119.1,112.2,111.7,99.5,75.3,70.2,57.9,55.8,41.2,41.0,38.4,37.3,34.1$, 26.6, 21.6, 16.9, 15.3 ppm; HRMS (ESI): calcd for $\mathrm{C}_{29} \mathrm{H}_{38} \mathrm{O}_{6} \mathrm{Na}^{+}[\mathrm{M}+\mathrm{Na}]^{+}$505.2561, found 505.2560.

C7-epi-18: m.p. $116-118{ }^{\circ} \mathrm{C}$ (hexanes/EtOAc); $R_{\mathrm{f}}=0.50$ (silica gel, hexanes:EtOAc $2: 1) ;[\alpha]_{\mathrm{D}}^{20}=+0.7\left(\mathrm{c}=0.5, \mathrm{CHCl}_{3}\right) ;{ }^{1} \mathrm{H} \mathrm{NMR}\left(400 \mathrm{MHz}, \mathrm{CDCl}_{3}\right) \delta=8.11-8.08(\mathrm{~m}, 2$ H), $7.57(\mathrm{t}, J=7.4 \mathrm{~Hz}, 1 \mathrm{H}), 7.47(\mathrm{t}, J=7.6 \mathrm{~Hz}, 2 \mathrm{H}), 6.79(\mathrm{~s}, 1 \mathrm{H}), 6.68(\mathrm{~d}, J=1.3 \mathrm{~Hz}$, $1 \mathrm{H}), 5.12-5.04(\mathrm{~m}, 4 \mathrm{H}), 4.98(\mathrm{~d}, J=6.1 \mathrm{~Hz}, 1 \mathrm{H}), 4.88(\mathrm{dd}, J=17.4,1.3 \mathrm{~Hz}, 1 \mathrm{H})$, $4.78(\mathrm{dd}, J=10.6,1.3 \mathrm{~Hz}, 1 \mathrm{H}), 3.88(\mathrm{~s}, 3 \mathrm{H}), 3.47(\mathrm{~s}, 3 \mathrm{H}), 2.66(\mathrm{~d}, J=4.1 \mathrm{~Hz}, 1 \mathrm{H})$, $2.33(\mathrm{~s}, 3 \mathrm{H}), 1.90-1.63(\mathrm{~m}, 5 \mathrm{H}), 1.57-1.45(\mathrm{~m}, 2 \mathrm{H}), 1.19(\mathrm{~d}, J=6.8 \mathrm{~Hz}, 3 \mathrm{H}), 1.05-$ $1.00(\mathrm{~m}, 1 \mathrm{H}), 0.98 \mathrm{ppm}(\mathrm{s}, 3 \mathrm{H}) ;{ }^{13} \mathrm{C} \mathrm{NMR}\left(100 \mathrm{MHz}, \mathrm{CDCl}_{3}\right) \delta=166.3,151.4,149.8$, $141.9,137.2,133.9,132.8,131.0,129.9$ (2C), 128.5 (2C), 120.8, 112.2, 110.8, 99.3, 75.6, 69.0, 57.8, 55.8, 41.0, 39.9, 38.0, 36.9, 33.8, 26.3, 21.6, 17.1, 14.8 ppm; HRMS (ESI): calcd for $\mathrm{C}_{29} \mathrm{H}_{38} \mathrm{O}_{6} \mathrm{Na}^{+}[\mathrm{M}+\mathrm{Na}]^{+}$505.2561, found 505.2566.

\section{Recycle of alcohol C7-epi-18:}

To a stirred solution of alcohol C7-epi-18 (11.0 g, $22.8 \mathrm{mmol}, 1.0$ equiv) and silica gel (5.0 g) in $\mathrm{CH}_{2} \mathrm{Cl}_{2}(50 \mathrm{~mL})$ at room temperature was added PDC (17.2 g, $45.6 \mathrm{mmol}$, 2.0 equiv). The resulting mixture was stirred at room temperature for $2 \mathrm{~h}$ before filtered 
through a short pad of celite. The filtrate was concentrated in vacuo. Flash column chromatography (silica gel, hexanes:EtOAc 8:1) afforded the corresponding ketone as a white solid.

To a stirred solution of ketone (obtained above) in $\mathrm{EtOH}(50 \mathrm{~mL})$ at $0{ }^{\circ} \mathrm{C}$ was added $\mathrm{NaBH}_{4}\left(1.0 \mathrm{~g}, 27 \mathrm{mmol}, 1.0\right.$ equiv). The resulting mixture was stirred at $0{ }^{\circ} \mathrm{C}$ for 30 min before quenched with acetone ( $5 \mathrm{~mL})$ and $\mathrm{NH}_{4} \mathrm{Cl}(20 \mathrm{~mL}$, sat. aq.). The layers were separated, and the aqueous layer was extracted with $\mathrm{CH}_{2} \mathrm{Cl}_{2}(3 \times 20 \mathrm{~mL})$. The combined organic layers were washed with brine $(20 \mathrm{~mL}$, sat. aq. $)$, dried $\left(\mathrm{Na}_{2} \mathrm{SO}_{4}\right)$ and concentrated in vacuo. Flash column chromatography (silica gel, hexanes:EtOAc 6:1) afforded 18 (6.7 g, 61\% over two steps), along with the recovered C7-epi-18 (2.1 g, 19\% over two steps).

\section{Preparation of tetracyclic diketone 13:}
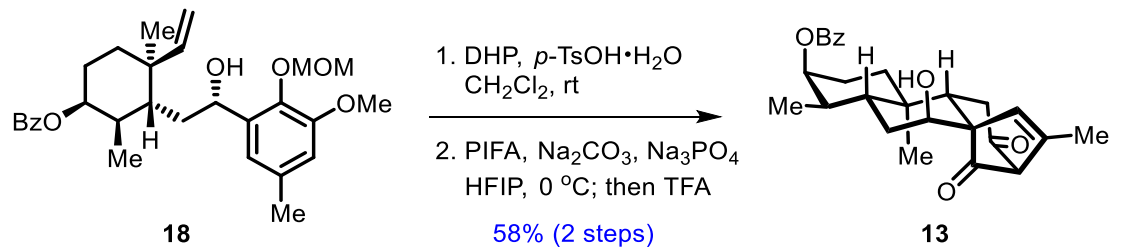

To a stirred solution of alcohol 18 (2.3 g, $5.3 \mathrm{mmol}, 1.0$ equiv) in $\mathrm{CH}_{2} \mathrm{Cl}_{2}(80 \mathrm{~mL})$ at room temperature were sequentially added DHP (1.2 mL, $13 \mathrm{mmol}, 2.5$ equiv) and $p$ - $\mathrm{TsOH} \cdot \mathrm{H}_{2} \mathrm{O}(1.0 \mathrm{~g}, 5.3 \mathrm{mmol}, 1.0$ equiv). The resulting mixture was stirred at room temperature for $3 \mathrm{~h}$ before quenched with $\mathrm{NaHCO}_{3}(20 \mathrm{~mL}$, sat. aq.). The layers were separated, and the aqueous layer was extracted with $\mathrm{CH}_{2} \mathrm{Cl}_{2}(3 \times 10 \mathrm{~mL})$. The combined organic layers were dried $\left(\mathrm{Na}_{2} \mathrm{SO}_{4}\right)$ and concentrated in vacuo to afford the crude vinylphenol as a yellow oil, which was used directly without further purification.

To a stirred solution of vinylphenol (crude, obtained above) in HFIP $(200 \mathrm{~mL})$ at 0 
${ }^{\circ} \mathrm{C}$ were added $\mathrm{Na}_{2} \mathrm{CO}_{3}\left(1.7 \mathrm{~g}, 16 \mathrm{mmol}, 3.0\right.$ equiv), $\mathrm{Na}_{3} \mathrm{PO}_{4}(1.7 \mathrm{~g}, 11 \mathrm{mmol}, 2.0$ equiv) and PIFA (2.8 g, $6.4 \mathrm{mmol}, 1.2$ equiv). The resulting mixture was stirred at $0{ }^{\circ} \mathrm{C}$ for 20 min before TFA (9.8 mL, 0.13 mol, 25 equiv) was added. The reaction was stirred at room temperature for an additional 30 min before quenched with $\mathrm{NaHCO}_{3}(200 \mathrm{~mL}$, sat. aq.) and $\mathrm{Na}_{2} \mathrm{~S}_{2} \mathrm{O}_{3}(120 \mathrm{~mL}$, sat. aq.). The layers were separated, and the aqueous layer was extracted with $\mathrm{Et}_{2} \mathrm{O}(3 \times 100 \mathrm{~mL})$. The combined organic layers were dried $\left(\mathrm{Na}_{2} \mathrm{SO}_{4}\right)$ and concentrated in vacuo. Flash column chromatography (silica gel, hexanes:EtOAc 4:1) afforded tetracyclic diketone $\mathbf{1 3}$ (1.3 g, 58\% over two steps) as a white solid. 13: m.p. $218-220{ }^{\circ} \mathrm{C}$ (hexanes/EtOAc); $R_{\mathrm{f}}=0.50$ (silica gel, hexanes:EtOAc 2:1); $[\alpha]_{\mathrm{D}}{ }^{20}=+6.4\left(\mathrm{c}=0.5, \mathrm{CHCl}_{3}\right) ;{ }^{1} \mathrm{H} \mathrm{NMR}\left(400 \mathrm{MHz}, \mathrm{CDCl}_{3}\right) \delta=$ $8.02(\mathrm{~d}, J=8.0 \mathrm{~Hz}, 2 \mathrm{H}), 7.53(\mathrm{t}, J=7.4 \mathrm{~Hz}, 1 \mathrm{H}), 7.41(\mathrm{t}, J=7.7 \mathrm{~Hz}, 2 \mathrm{H}), 6.36-6.27$ (m, $1 \mathrm{H}), 5.21-5.12(\mathrm{~m}, 1 \mathrm{H}), 4.20(\mathrm{~s}, 1 \mathrm{H}), 3.51(\mathrm{~s}, 1 \mathrm{H}), 2.86(\mathrm{dd}, J=17.7,10.6 \mathrm{~Hz}$ $1 \mathrm{H}), 2.43(\mathrm{~d}, J=17.7 \mathrm{~Hz}, 1 \mathrm{H}), 2.27(\mathrm{~d}, J=10.5 \mathrm{~Hz}, 1 \mathrm{H}), 2.16(\mathrm{~s}, 1 \mathrm{H}), 2.08(\mathrm{td}, J=$ 12.2, 3.7 Hz, $1 \mathrm{H}), 1.88-1.77(\mathrm{~m}, 6 \mathrm{H}), 1.76-1.62(\mathrm{~m}, 2 \mathrm{H}), 1.50(\mathrm{~d}, J=13.0 \mathrm{~Hz}, 1 \mathrm{H})$, $1.30(\mathrm{td}, J=13.2,3.8 \mathrm{~Hz}, 1 \mathrm{H}), 0.88(\mathrm{~d}, J=6.7 \mathrm{~Hz}, 3 \mathrm{H}), 0.63 \mathrm{ppm}(\mathrm{s}, 3 \mathrm{H}) ;{ }^{13} \mathrm{C} \mathrm{NMR}$ $\left(100 \mathrm{MHz}, \mathrm{CDCl}_{3}\right) \delta=205.2,204.5,166.2,140.8,133.0,132.1,130.7,129.6(2 \mathrm{C})$, 128.5 (2C), 75.3, 74.6, 66.0, 58.0, 42.9, 38.4, 37.4, 35.9, 33.7, 33.6, 29.9, 26.0, 17.5, 16.2, 10.8 ppm; HRMS (ESI): calcd for $\mathrm{C}_{26} \mathrm{H}_{30} \mathrm{NaO}_{5}{ }^{+}[\mathrm{M}+\mathrm{Na}]^{+} 445.1985$, found 445.1991. 
Preparation of tricycle 21:

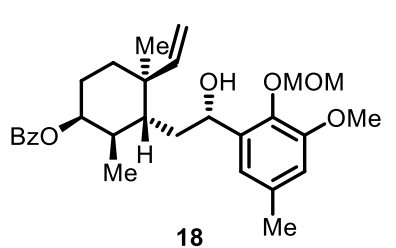

18

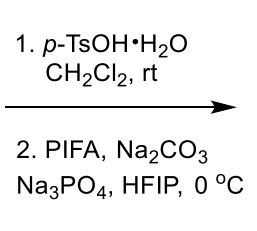

$\mathrm{Na}_{3} \mathrm{PO}_{4}, \mathrm{HFIP}, 0^{\circ} \mathrm{C}$

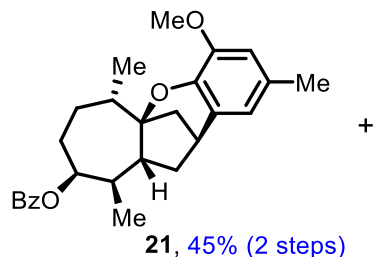

$21,45 \%$ (2 steps)

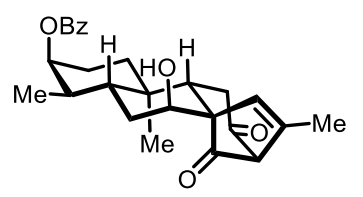

13, $10 \%$ (2 steps)

To a stirred solution of alcohol $18\left(0.11 \mathrm{~g}, 0.23 \mathrm{mmol}, 1.0\right.$ equiv) in $\mathrm{CH}_{2} \mathrm{Cl}_{2}$ (10 $\mathrm{mL})$ at room temperature was $p-\mathrm{TsOH} \cdot \mathrm{H}_{2} \mathrm{O}(23 \mathrm{mg}, 0.12 \mathrm{mmol}, 0.5$ equiv). The resulting mixture was stirred at room temperature for $3 \mathrm{~h}$ before quenched with $\mathrm{NaHCO}_{3}(5 \mathrm{~mL}$, sat. aq.). The layers were separated, and the aqueous layer was extracted with $\mathrm{CH}_{2} \mathrm{Cl}_{2}(3 \times 5 \mathrm{~mL})$. The combined organic layers were dried $\left(\mathrm{Na}_{2} \mathrm{SO}_{4}\right)$ and concentrated in vacuo to afford the crude vinylphenol as a yellow oil, which was used directly without further purification.

To a stirred solution of vinylphenol (crude, obtained above) in HFIP (10 $\mathrm{mL})$ at 0 ${ }^{\circ} \mathrm{C}$ were added $\mathrm{Na}_{2} \mathrm{CO}_{3}$ (73 mg, 0.69 mmol, 3.0 equiv), $\mathrm{Na}_{3} \mathrm{PO}_{4}(75 \mathrm{mg}, 0.46 \mathrm{mmol}, 2.0$ equiv) and PIFA ( $0.15 \mathrm{~g}, 0.34 \mathrm{mmol}, 1.2$ equiv). The resulting mixture was stirred at 0 ${ }^{\circ} \mathrm{C}$ for $20 \mathrm{~min}$ before quenched with $\mathrm{Na}_{2} \mathrm{~S}_{2} \mathrm{O}_{3}(5 \mathrm{~mL}$, sat. aq.). The layers were separated, and the aqueous layer was extracted with $\mathrm{Et}_{2} \mathrm{O}(3 \times 5 \mathrm{~mL})$. The combined organic layers were dried $\left(\mathrm{Na}_{2} \mathrm{SO}_{4}\right)$ and concentrated in vacuo. Flash column chromatography (silica gel, hexanes:EtOAc 4:1) afforded tricycle 21 (43 mg, 45\% over two steps) as a white solid, along with 13 (8.5 mg, 10\% over two steps). 21: m.p. $141-143{ }^{\circ} \mathrm{C}$ $\left(\mathrm{CH}_{2} \mathrm{Cl}_{2} / \mathrm{EtOAc}\right) ; R_{\mathrm{f}}=0.50$ (silica gel, hexanes:EtOAc 5:1); $[\alpha]_{\mathrm{D}}{ }^{20}=+35.0(\mathrm{c}=0.5$, $\left.\mathrm{CHCl}_{3}\right) ;{ }^{1} \mathrm{H} \mathrm{NMR}\left(400 \mathrm{MHz}, \mathrm{CDCl}_{3}\right) \delta=8.08(\mathrm{~d}, J=7.1 \mathrm{~Hz}, 2 \mathrm{H}), 7.53(\mathrm{t}, J=8.0 \mathrm{~Hz}$, $1 \mathrm{H}), 7.42(\mathrm{~d}, J=7.8 \mathrm{~Hz}, 2 \mathrm{H}), 6.60(\mathrm{~d}, J=2.0 \mathrm{~Hz}, 1 \mathrm{H}), 6.47(\mathrm{~d}, J=1.9 \mathrm{~Hz}, 1 \mathrm{H})$, 5.26-5.21 (m, $1 \mathrm{H}), 3.88$ (s, $3 \mathrm{H}), 3.00(\mathrm{t}, J=4.4 \mathrm{~Hz}, 1 \mathrm{H}), 2.70-2.52(\mathrm{~m}, 2 \mathrm{H}), 2.26$ (s, 
$3 \mathrm{H}), 2.26-2.19(\mathrm{~m}, 1 \mathrm{H}) 2.12-1.93(\mathrm{~m}, 3 \mathrm{H}), 1.93-1.81(\mathrm{~m}, 3 \mathrm{H}), 1.81-1.69(\mathrm{~m}, 1 \mathrm{H})$, 1.67-1.57 (m, $1 \mathrm{H}), 1.15(\mathrm{~d}, J=7.1 \mathrm{~Hz}, 3 \mathrm{H}), 1.04 \mathrm{ppm}(\mathrm{d}, J=7.0 \mathrm{~Hz}, 3 \mathrm{H}) ;{ }^{13} \mathrm{C} \mathrm{NMR}$ $\left(100 \mathrm{MHz}, \mathrm{CDCl}_{3}\right) \delta=166.1,148.2,140.8,132.9,130.9,130.1,130.0(2 \mathrm{C}), 128.4(2 \mathrm{C})$, $120.1,112.2,91.0,76.3,56.6,49.5,45.5,39.7,37.6,37.3$ (2C), 34.3, 29.7, 26.4, 21.0, 18.8, 18.5 ppm; HRMS (ESI): calcd for $\mathrm{C}_{27} \mathrm{H}_{32} \mathrm{NaO}_{4}{ }^{+}[\mathrm{M}+\mathrm{Na}]^{+} 443.2193$, found 443.2190.

Investigations on the ODI-(5+2) Cycloaddition/Pinacol-Type 1,2-Acyl Migration Cascade Reactions of C7-epi-18 and C7-Deoxy-14

a)
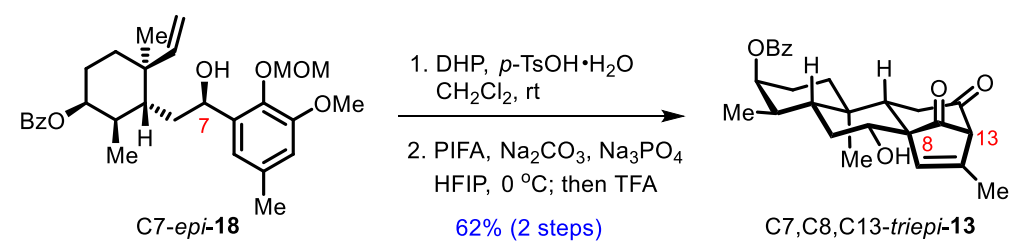

b)

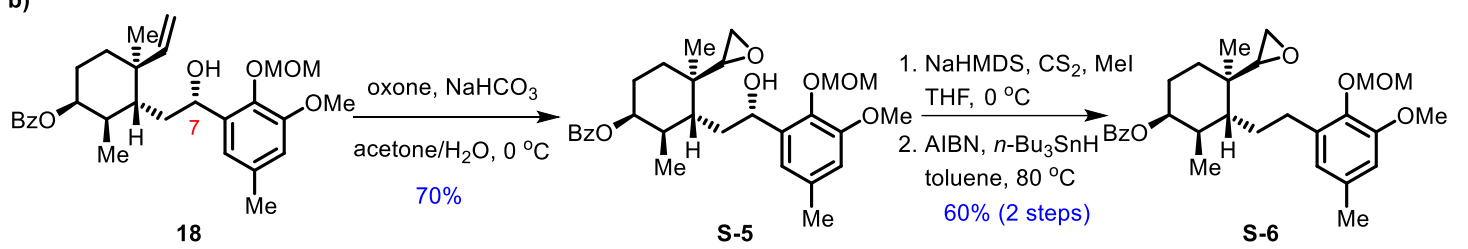
18 S-5

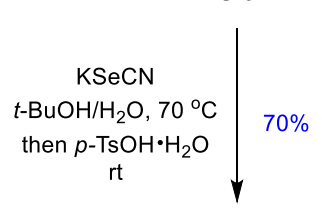

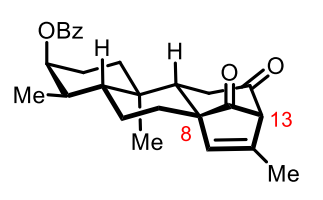

C7-deoxy-C8,C13-diepi-13, 55\%

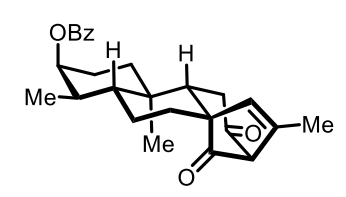

C7-deoxy-13, 8\%

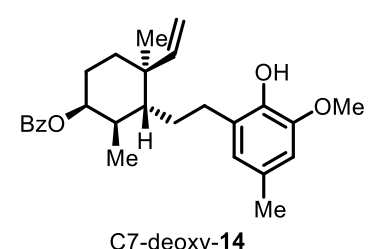

C7-deoxy-14

Scheme S2. Attempts of ODI-(5+2) cascades of a) C7-epi-18, and b) C7-deoxy-14 


\section{Preparation of tetracyclic diketone C7,C8,C13-triepi-13:}

To a stirred solution of alcohol C7-epi-18 (63 mg, $0.13 \mathrm{mmol}, 1.0$ equiv) in $\mathrm{CH}_{2} \mathrm{Cl}_{2}$ $(5 \mathrm{~mL})$ at room temperature were sequentially added DHP ( $30 \mu \mathrm{L}, 0.33 \mathrm{mmol}, 2.5$ equiv) and $p-\mathrm{TsOH} \cdot \mathrm{H}_{2} \mathrm{O}(26 \mathrm{mg}, 0.13 \mathrm{mmol}, 1.0$ equiv). The resulting mixture was stirred at room temperature for $3 \mathrm{~h}$ before quenched with $\mathrm{NaHCO}_{3}(5 \mathrm{~mL}$, sat. aq.). The layers were separated, and the aqueous layer was extracted with $\mathrm{CH}_{2} \mathrm{Cl}_{2}(3 \times 5 \mathrm{~mL})$. The combined organic layers were dried $\left(\mathrm{Na}_{2} \mathrm{SO}_{4}\right)$ and concentrated in vacuo to afford the crude vinylphenol as a white solid, which was used directly without further purification.

To a stirred solution of vinylphenol (crude, obtained above) in HFIP $(6 \mathrm{~mL})$ at $0{ }^{\circ} \mathrm{C}$ were added $\mathrm{Na}_{2} \mathrm{CO}_{3}$ (42 mg, 0.40 mmol, 3.0 equiv), $\mathrm{Na}_{3} \mathrm{PO}_{4}$ (43 mg, 0.26 mmol, 2.0 equiv) and PIFA (68 mg, $0.16 \mathrm{mmol}, 1.2$ equiv). The resulting mixture was stirred at 0 ${ }^{\circ} \mathrm{C}$ for $20 \mathrm{~min}$ before TFA $(0.25 \mathrm{~mL}, 3.3 \mathrm{mmol}, 25$ equiv $)$ was added. The resulting mixture was stirred at room temperature for 30 min before quenched with $\mathrm{NaHCO}_{3}(10$ mL, sat. aq.) and $\mathrm{Na}_{2} \mathrm{~S}_{2} \mathrm{O}_{3}(5 \mathrm{~mL}$, sat. aq.). The layers were separated, and the aqueous layer was extracted with $\mathrm{Et}_{2} \mathrm{O}(3 \times 5 \mathrm{~mL})$. The combined organic layers were dried $\left(\mathrm{Na}_{2} \mathrm{SO}_{4}\right)$ and concentrated in vасио. Flash column chromatography (silica gel, hexanes:EtOAc 4:1) afforded tetracyclic diketone C7,C8,C13-triepi-13 (34 mg, 62\% over two steps) as a white solid. C7,C8,C13-triepi-13: m.p. $150-152{ }^{\circ} \mathrm{C}$ (hexanes/EtOAc); $R_{\mathrm{f}}=0.25$ (silica gel, hexanes:EtOAc 2:1); $[\alpha]_{\mathrm{D}}{ }^{20}=-171.6(\mathrm{c}=0.5$, $\left.\mathrm{CHCl}_{3}\right) ;{ }^{1} \mathrm{H} \mathrm{NMR}\left(400 \mathrm{MHz}, \mathrm{CDCl}_{3}\right) \delta=8.00(\mathrm{~d}, J=7.6 \mathrm{~Hz}, 2 \mathrm{H}), 7.57(\mathrm{t}, J=7.4 \mathrm{~Hz}$, $1 \mathrm{H}), 7.46(\mathrm{t}, J=7.6 \mathrm{~Hz}, 2 \mathrm{H}), 6.20(\mathrm{~s}, 1 \mathrm{H}), 5.25-5.16(\mathrm{~m}, 1 \mathrm{H}), 4.32(\mathrm{~d}, J=11.3 \mathrm{~Hz}$, $1 \mathrm{H}), 3.58(\mathrm{~s}, 1 \mathrm{H}), 2.67(\mathrm{dd}, J=16.9,11.8 \mathrm{~Hz}, 1 \mathrm{H}), 2.38(\mathrm{dd}, J=16.9,6.1 \mathrm{~Hz}, 1 \mathrm{H})$, 
2.10-1.97 (m, $2 \mathrm{H}), 1.92$ (s, $3 \mathrm{H}), 1.89-1.78$ (m, $2 \mathrm{H}), 1.71(\mathrm{dd}, J=21.4,12.1 \mathrm{~Hz}, 1 \mathrm{H})$, 1.63-1.52 (m, $2 \mathrm{H}), 1.44-1.25(\mathrm{~m}, 3 \mathrm{H}), 0.98(\mathrm{~d}, J=6.7 \mathrm{~Hz}, 3 \mathrm{H}), 0.93$ ppm (s, $3 \mathrm{H})$; ${ }^{13} \mathrm{C} \mathrm{NMR}\left(100 \mathrm{MHz}, \mathrm{CDCl}_{3}\right) \delta=205.9,201.2,166.1,138.2,133.2,130.7,129.6(2 \mathrm{C})$, 128.6 (2C), 128.3, 74.2, 74.1, 67.2, 62.5, 45.9, 44.2, 37.5, 36.1, 34.2, 32.6, 29.7, 26.0, 17.4, 16.4, 12.4 ppm; HRMS (ESI): calcd for $\mathrm{C}_{26} \mathrm{H}_{30} \mathrm{NaO}_{5}{ }^{+}[\mathrm{M}+\mathrm{Na}]^{+} 445.1985$, found 445.1987.

\section{Preparation of epoxide S-5:}

To a stirred solution of alcohol 18 (1.2 g, $2.5 \mathrm{mmol}, 1.0$ equiv) and $\mathrm{NaHCO}_{3}(1.0$ $\mathrm{g}, 12 \mathrm{mmol}, 4.8$ equiv) in acetone $/ \mathrm{H}_{2} \mathrm{O}(1: 1,20 \mathrm{~mL})$ at $0{ }^{\circ} \mathrm{C}$ was added oxone $(1.5 \mathrm{~g}$, $2.5 \mathrm{mmol}, 1.0$ equiv). The resulting mixture was stirred at $0{ }^{\circ} \mathrm{C}$ for $12 \mathrm{~h}$ before diluted with EtOAc (10 mL) and quenched with $\mathrm{Na}_{2} \mathrm{~S}_{2} \mathrm{O}_{3}(10 \mathrm{~mL}$, sat. aq.). The layers were separated, and the aqueous layer was extracted with EtOAc $(3 \times 10 \mathrm{~mL})$. The combined organic layers were dried $\left(\mathrm{Na}_{2} \mathrm{SO}_{4}\right)$ and concentrated in vacuo. Flash column chromatography (silica gel, hexanes:EtOAc 4:1) afforded epoxide S-5 (0.87 g, 70\%) as a white solid. S-5: m.p. $120-122{ }^{\circ} \mathrm{C}$ (hexanes/EtOAc); $R_{\mathrm{f}}=0.30$ (silica gel, hexanes:EtOAc 2:1); $[\alpha]_{\mathrm{D}}^{20}=+4.0\left(\mathrm{c}=0.5, \mathrm{CHCl}_{3}\right) ;{ }^{1} \mathrm{H} \mathrm{NMR}\left(400 \mathrm{MHz}, \mathrm{CDCl}_{3}\right) \delta=$ $8.13(\mathrm{~d}, J=7.3 \mathrm{~Hz}, 2 \mathrm{H}), 7.59(\mathrm{t}, J=7.3 \mathrm{~Hz}, 1 \mathrm{H}), 7.48(\mathrm{t}, J=7.6 \mathrm{~Hz}, 2 \mathrm{H}), 6.80(\mathrm{~s}, 1$ H), 6.66 (s, $1 \mathrm{H}), 5.14-5.12(\mathrm{~m}, 2 \mathrm{H}), 5.07(\mathrm{dd}, J=10.0,4.7 \mathrm{~Hz}, 1 \mathrm{H}), 4.94(\mathrm{~d}, J=6.1$ Hz, $1 \mathrm{H}), 3.86$ (s, $3 \mathrm{H}), 3.44$ (s, $3 \mathrm{H}), 2.92$ (s, $1 \mathrm{H}), 2.67$ (dd, J = 4.3, 2.9 Hz, $1 \mathrm{H}), 2.47$ (t, $J=4.2 \mathrm{~Hz}, 1 \mathrm{H}), 2.31(\mathrm{~s}, 3 \mathrm{H}), 1.92-1.80(\mathrm{~m}, 4 \mathrm{H}), 1.75-1.69$ (m, $2 \mathrm{H}), 1.58-1.49$ (m, $2 \mathrm{H}), 1.31(\mathrm{~d}, J=13.3 \mathrm{~Hz}, 1 \mathrm{H}), 1.21(\mathrm{~d}, J=6.8 \mathrm{~Hz}, 3 \mathrm{H}), 0.60 \mathrm{ppm}(\mathrm{s}, 3 \mathrm{H}) ;{ }^{13} \mathrm{C}$ NMR $\left(100 \mathrm{MHz}, \mathrm{CDCl}_{3}\right) \delta=166.2,151.5,141.8,137.5,134.1,132.8,130.9,129.9$ 
(2C), 128.5 (2C), 120.1, 112.5, 99.3, 75.1, 69.9, 60.3, 57.8, 55.8, 45.7, 38.9, 38.6, 37.5, 37.1, 30.5, 25.9, 21.6, 16.8, 10.1 ppm; HRMS (ESI): calcd for $\mathrm{C}_{29} \mathrm{H}_{38} \mathrm{O}_{7} \mathrm{Na}^{+}[\mathrm{M}+\mathrm{Na}]^{+}$ 521.2510, found 521.2515.

\section{Preparation of MOM ether S-6:}

To a stirred solution of epoxide S-5 (0.60 g, $1.2 \mathrm{mmol}, 1.0$ equiv) and $\mathrm{CS}_{2}(0.73$ $\mathrm{mL}, 12 \mathrm{mmol}, 10$ equiv) in THF $(10 \mathrm{~mL})$ at $0{ }^{\circ} \mathrm{C}$ was added NaHMDS (2.0 $\mathrm{M}$ in THF, $1.8 \mathrm{~mL}, 3.6 \mathrm{mmol}, 3.0$ equiv). The resulting mixture was stirred at $0{ }^{\circ} \mathrm{C}$ for $30 \mathrm{~min}$ before MeI ( $0.37 \mathrm{~mL}, 6.0 \mathrm{mmol}, 5.0$ equiv) was added. The reaction was stirred at $0{ }^{\circ} \mathrm{C}$ for an additional $10 \mathrm{~min}$ before quenched with $\mathrm{NH}_{4} \mathrm{Cl}(5 \mathrm{~mL}$, sat. aq.). The layers were separated, and the aqueous layer was extracted with EtOAc $(3 \times 5 \mathrm{~mL})$. The combined organic layers were dried $\left(\mathrm{Na}_{2} \mathrm{SO}_{4}\right)$ and concentrated in vacuo to afford the crude xanthate as a yellow oil, which was used directly without further purification.

To a stirred solution of xanthate (crude, obtained above) in toluene $(10 \mathrm{~mL}$, degassed) at room temperature were sequentially added AIBN (39 mg, 0.24 mmol, 0.2 equiv) and $n-\mathrm{Bu}_{3} \mathrm{SnH}(0.96 \mathrm{~mL}, 3.6 \mathrm{mmol}, 3.0$ equiv). The resulting mixture was warmed to $80^{\circ} \mathrm{C}$ and stirred for $2 \mathrm{~h}$ before cooled to room temperature and concentrated in vacuo. Flash column chromatography (silica gel, hexanes:EtOAc 4:1) afforded MOM ether S-6 (0.35 g, 60\% over two steps) as a white solid. S-6: m.p. $114-116{ }^{\circ} \mathrm{C}$ (hexanes/EtOAc); $R_{\mathrm{f}}=0.55$ (silica gel, hexanes:EtOAc 4:1); $[\alpha]_{\mathrm{D}}^{20}=+3.1(\mathrm{c}=0.5$,

$\left.\mathrm{CHCl}_{3}\right) ;{ }^{1} \mathrm{H} \mathrm{NMR}\left(400 \mathrm{MHz}, \mathrm{CDCl}_{3}\right) \delta=8.12-8.10(\mathrm{~m}, 2 \mathrm{H}), 7.59(\mathrm{t}, J=7.4 \mathrm{~Hz}, 1 \mathrm{H})$, $7.49(\mathrm{t}, J=7.5 \mathrm{~Hz}, 2 \mathrm{H}), 6.58(\mathrm{~d}, J=3.4 \mathrm{~Hz}, 2 \mathrm{H}), 5.20(\mathrm{~d}, J=2.2 \mathrm{~Hz}, 1 \mathrm{H}), 5.04(\mathrm{dd}$, $J=14.3,5.7 \mathrm{~Hz}, 2 \mathrm{H}), 3.82(\mathrm{~s}, 3 \mathrm{H}), 3.52(\mathrm{~s}, 3 \mathrm{H}), 2.82-2.63(\mathrm{~m}, 4 \mathrm{H}), 2.51-2.50(\mathrm{~m}, 1$ 
H), $2.30(\mathrm{~s}, 3 \mathrm{H}), 1.95-1.91(\mathrm{~m}, 1 \mathrm{H}), 1.84-1.61(\mathrm{~m}, 5 \mathrm{H}), 1.47-1.32(\mathrm{~m}, 2 \mathrm{H}), 1.15$ (d, $J=6.7 \mathrm{~Hz}, 3 \mathrm{H}), 0.68 \mathrm{ppm}(\mathrm{s}, 3 \mathrm{H}) ;{ }^{13} \mathrm{C} \mathrm{NMR}\left(100 \mathrm{MHz}, \mathrm{CDCl}_{3}\right) \delta=166.3,151.9$, $141.9,136.3,133.8,133.0,131.0,129.7(2 \mathrm{C}), 128.6(2 \mathrm{C}), 122.7,111.2,99.1,74.9,60.2$, 57.7, 55.7, 45.2, 42.5, 37.5, 37.1, 31.9, 31.0, 30.1, 26.0, 21.5, 16.7, 11.2 ppm; HRMS (ESI): calcd for $\mathrm{C}_{29} \mathrm{H}_{38} \mathrm{O}_{6} \mathrm{Na}^{+}[\mathrm{M}+\mathrm{Na}]^{+}$505.2561, found 505.2566.

\section{Preparation of vinylphenol C7-deoxy-14:}

To a stirred solution of MOM ether $\mathbf{S}-6(0.20 \mathrm{~g}, 0.41 \mathrm{mmol}, 1.0$ equiv) in $t$ $\mathrm{BuOH} / \mathrm{H}_{2} \mathrm{O}(9: 1,5 \mathrm{~mL})$ at room temperature was added $\mathrm{KSeCN}(0.14 \mathrm{~g}, 1.0 \mathrm{mmol}, 2.5$ equiv). The resulting mixture was warmed to $70{ }^{\circ} \mathrm{C}$ and stirred for $36 \mathrm{~h}$ before $p$ $\mathrm{TsOH} \cdot \mathrm{H}_{2} \mathrm{O}(0.19 \mathrm{~g}, 1.0 \mathrm{mmol}, 2.5$ equiv $)$ was added. The resulting mixture was stirred at room temperature for $2 \mathrm{~h}$ before diluted with EtOAc $(5 \mathrm{~mL})$ and quenched with $\mathrm{NaHCO}_{3}$ (15 mL, sat. aq.). The layers were separated, and the aqueous layer was extracted with EtOAc $(3 \times 5 \mathrm{~mL})$. The combined organic layers were dried $\left(\mathrm{Na}_{2} \mathrm{SO}_{4}\right)$ and concentrated in vacuo. Flash column chromatography (silica gel, hexanes:EtOAc 10:1) afforded vinylphenol C7-deoxy-14 (0.12 g, 70\%) as a white solid. C7-deoxy-14: m.p. $107-109{ }^{\circ} \mathrm{C}$ (hexanes/EtOAc); $R_{\mathrm{f}}=0.50$ (silica gel, hexanes:EtOAc 10:1); $[\alpha]_{\mathrm{D}}{ }^{20}$ $=+5.0\left(\mathrm{c}=0.5, \mathrm{CHCl}_{3}\right) ;{ }^{1} \mathrm{H} \mathrm{NMR}\left(400 \mathrm{MHz}, \mathrm{CDCl}_{3}\right) \delta=8.12-8.09(\mathrm{~m}, 2 \mathrm{H}), 7.60(\mathrm{t}$, $J=7.4 \mathrm{~Hz}, 1 \mathrm{H}), 7.50(\mathrm{t}, J=7.6 \mathrm{~Hz}, 2 \mathrm{H}), 6.55(\mathrm{~d}, J=4.1 \mathrm{~Hz}, 2 \mathrm{H}), 5.71(\mathrm{dd}, J=17.5$, $10.7 \mathrm{~Hz}, 1 \mathrm{H}), 5.50(\mathrm{~s}, 1 \mathrm{H}), 5.22(\mathrm{~d}, J=2.2 \mathrm{~Hz}, 1 \mathrm{H}), 5.03-4.96(\mathrm{~m}, 2 \mathrm{H}), 3.86(\mathrm{~s}, 3 \mathrm{H})$, $2.73(\mathrm{ddd}, J=13.1,11.4,4.7 \mathrm{~Hz}, 1 \mathrm{H}), 2.52(\mathrm{ddd}, J=13.2,10.7,6.7 \mathrm{~Hz}, 1 \mathrm{H}), 2.29$ (s, $3 \mathrm{H}), 1.92(\mathrm{dd}, J=13.4,2.9 \mathrm{~Hz}, 1 \mathrm{H}), 1.88-1.73(\mathrm{~m}, 3 \mathrm{H}), 1.70-1.63(\mathrm{~m}, 1 \mathrm{H}), 1.58-$ $1.52(\mathrm{~m}, 1 \mathrm{H}), 1.38$ (ddd, $J=19.1,10.7,5.3 \mathrm{~Hz}, 1 \mathrm{H}), 1.20$ (d, $J=2.9 \mathrm{~Hz}, 1 \mathrm{H}), 1.16$ (d, 
$J=6.8 \mathrm{~Hz}, 3 \mathrm{H}), 1.01 \mathrm{ppm}(\mathrm{s}, 3 \mathrm{H}) ;{ }^{13} \mathrm{C} \mathrm{NMR}\left(100 \mathrm{MHz}, \mathrm{CDCl}_{3}\right) \delta=166.3,149.7$, $146.2,141.2,132.9,131.1,129.7$ (2C), 128.6, 128.5 (2C), 128.3, 122.8, 111.2, 109.3, 75.4, 56.1, 44.4, 41.2, 37.0, 34.1, 31.4, 30.4, 26.5, 21.3, 17.0, 15.5 ppm; HRMS (ESI): calcd for $\mathrm{C}_{27} \mathrm{H}_{34} \mathrm{O}_{4} \mathrm{Na}^{+}[\mathrm{M}+\mathrm{Na}]^{+} 445.2349$, found 445.2355.

\section{Preparation of tetracyclic diketones C7-deoxy-13 and C7-deoxy-C8,C13-diepi-13:}

To a stirred solution of vinylphenol C7-deoxy-14 (0.10 g, 0.24 mmol, 1.0 equiv) in HFIP $(10 \mathrm{~mL})$ at $0{ }^{\circ} \mathrm{C}$ was added PIFA $(0.12 \mathrm{~g}, 0.29 \mathrm{mmol}, 1.2$ equiv). The resulting mixture was stirred at $0{ }^{\circ} \mathrm{C}$ for 20 min before diluted with $\mathrm{Et}_{2} \mathrm{O}(5 \mathrm{~mL})$ and quenched with $\mathrm{Na}_{2} \mathrm{~S}_{2} \mathrm{O}_{3}$ (5 mL, sat. aq.). The layers were separated, and the aqueous layer was extracted with $\mathrm{Et}_{2} \mathrm{O}(3 \times 5 \mathrm{~mL})$. The combined organic layers were dried $\left(\mathrm{Na}_{2} \mathrm{SO}_{4}\right)$ and concentrated in vacuo. Flash column chromatography (silica gel, hexanes:EtOAc 4:1) afforded tetracyclic diketones C7-deoxy-13 (7.8 mg, 8\%) and C7-deoxy-C8,C13-diepi13 (54 mg, 55\%) as white solids. C7-deoxy-13: m.p. $196-198{ }^{\circ} \mathrm{C}$ (hexanes/EtOAc); $R_{\mathrm{f}}$ $=0.45$ (silica gel, hexanes:EtOAc 4:1); $[\alpha]_{\mathrm{D}}{ }^{20}=+5.5\left(\mathrm{c}=0.5, \mathrm{CHCl}_{3}\right) ;{ }^{1} \mathrm{H}$ NMR $(400$ $\left.\mathrm{MHz}, \mathrm{CDCl}_{3}\right) \delta=8.07-8.02(\mathrm{~m}, 2 \mathrm{H}), 7.60-7.55(\mathrm{~m}, 1 \mathrm{H}), 7.47(\mathrm{t}, J=7.6 \mathrm{~Hz}, 2 \mathrm{H})$, $5.84(\mathrm{~d}, J=1.6 \mathrm{~Hz}, 1 \mathrm{H}), 5.17(\mathrm{~d}, J=2.6 \mathrm{~Hz}, 1 \mathrm{H}), 3.52(\mathrm{~s}, 1 \mathrm{H}), 2.92(\mathrm{dd}, J=17.8$, $10.7 \mathrm{~Hz}, 1 \mathrm{H}), 2.48(\mathrm{~d}, J=17.8 \mathrm{~Hz}, 1 \mathrm{H}), 2.20(\mathrm{dd}, J=9.8,2.2 \mathrm{~Hz}, 1 \mathrm{H}), 1.90(\mathrm{~d}, J=$ $10.7 \mathrm{~Hz}, 2 \mathrm{H}), 1.83$ (d, J = 1.5 Hz, $3 \mathrm{H}), 1.80-1.69$ (m, $3 \mathrm{H}), 1.59-1.44$ (m, $4 \mathrm{H}), 1.32-$ $1.23(\mathrm{~m}, 1 \mathrm{H}), 0.94(\mathrm{~d}, J=6.7 \mathrm{~Hz}, 3 \mathrm{H}), 0.70 \mathrm{ppm}(\mathrm{s}, 3 \mathrm{H}) ;{ }^{13} \mathrm{C} \mathrm{NMR}\left(100 \mathrm{MHz}, \mathrm{CDCl}_{3}\right)$ $\delta=206.7,204.3,166.2,140.3,135.6,133.0,131.0,129.6(2 \mathrm{C}), 128.5(2 \mathrm{C}), 75.3,74.8$, 53.9, 47.9, 45.6, 38.5, 36.5, 34.4, 34.0, 29.0, 26.1, 21.4, 17.4, 16.3, 11.2 ppm; HRMS (ESI): calcd for $\mathrm{C}_{26} \mathrm{H}_{30} \mathrm{O}_{4} \mathrm{Na}^{+}[\mathrm{M}+\mathrm{Na}]^{+}$429.2036, found 429.2042. 
C7-deoxy-C8,C13-diepi-13: m.p. $164-166{ }^{\circ} \mathrm{C}$ (hexanes/EtOAc); $R_{\mathrm{f}}=0.40$ (silica gel, hexanes:EtOAc 4:1); $[\alpha]_{\mathrm{D}}{ }^{20}=-150.0\left(\mathrm{c}=0.5, \mathrm{CHCl}_{3}\right) ;{ }^{1} \mathrm{H}$ NMR $(400 \mathrm{MHz}$, acetone$\left.d_{6}\right) \delta=8.11-8.07(\mathrm{~m}, 2 \mathrm{H}), 7.65-7.61(\mathrm{~m}, 1 \mathrm{H}), 7.51(\mathrm{t}, J=7.6 \mathrm{~Hz}, 2 \mathrm{H}), 6.28(\mathrm{~d}, J=$ $1.6 \mathrm{~Hz}, 1 \mathrm{H}), 5.18(\mathrm{q}, J=2.7 \mathrm{~Hz}, 1 \mathrm{H}), 3.39(\mathrm{~s}, 1 \mathrm{H}), 2.73(\mathrm{dd}, J=16.7,12.0 \mathrm{~Hz}, 1 \mathrm{H})$, $2.32(\mathrm{dd}, J=16.7,5.9 \mathrm{~Hz}, 1 \mathrm{H}), 2.00(\mathrm{dd}, J=13.9,4.8 \mathrm{~Hz}, 1 \mathrm{H}), 1.90-1.79(\mathrm{~m}, 7 \mathrm{H})$, 1.66-1.57 (m, $2 \mathrm{H}), 1.54(\mathrm{dd}, J=12.0,6.0 \mathrm{~Hz}, 1 \mathrm{H}), 1.46-1.37(\mathrm{~m}, 3 \mathrm{H}), 1.01(\mathrm{~s}, 3 \mathrm{H})$, $0.96 \mathrm{ppm}(\mathrm{d}, J=6.8 \mathrm{~Hz}, 3 \mathrm{H}) ;{ }^{13} \mathrm{C} \mathrm{NMR}\left(100 \mathrm{MHz}\right.$, acetone- $\left.d_{6}\right) \delta=207.3,201.9,166.3$, 138.0, 133.7, 132.1, 131.8, 130.2 (2C), 129.4 (2C), 75.3, 74.9, 57.0, 47.6, 46.6, 38.3, 36.7, 34.9, 33.3, 27.5, 26.6, 21.3, 16.8, 16.6, 12.5 ppm; HRMS (ESI): calcd for $\mathrm{C}_{26} \mathrm{H}_{30} \mathrm{O}_{4} \mathrm{Na}^{+}[\mathrm{M}+\mathrm{Na}]^{+}$429.2036, found 429.2033.

\section{Preparation of tetracycle 22:}

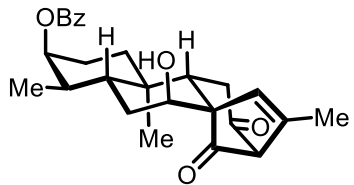

13

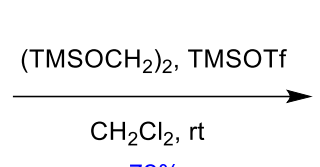

$72 \%$

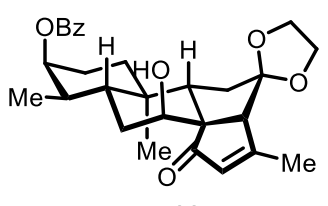

22

To a stirred solution of diketone $\mathbf{1 3}$ (4.8 g, $11 \mathrm{mmol}, 1.0$ equiv) in $\mathrm{CH}_{2} \mathrm{Cl}_{2}$ (110 $\mathrm{mL})$ at room temperature were sequentially added $\left(\mathrm{CH}_{2} \mathrm{OTMS}\right)_{2}(8.3 \mathrm{~mL}, 34 \mathrm{mmol}, 3.1$ equiv) and TMSOTf (2.2 mL, $13 \mathrm{mmol}, 1.2$ equiv). The resulting mixture was stirred at room temperature for $5 \mathrm{~min}$ before quenched with $\mathrm{NaHCO}_{3}(50 \mathrm{~mL}$, sat. aq.). The layers were separated, and the aqueous layer was extracted with $\mathrm{CH}_{2} \mathrm{Cl}_{2}(3 \times 50 \mathrm{~mL})$. The combined organic layers were dried $\left(\mathrm{Na}_{2} \mathrm{SO}_{4}\right)$ and concentrated in vacuo. Flash column chromatography (silica gel, hexanes:EtOAc 3:1) afforded tetracycle 22 (3.8 g, $72 \%$ ) as a white solid. 22: m.p. $185-187{ }^{\circ} \mathrm{C}$ (hexanes/EtOAc); $R_{\mathrm{f}}=0.20$ (silica gel, hexanes:EtOAc 2:1); $[\alpha]_{\mathrm{D}}{ }^{20}=+2.5\left(\mathrm{c}=0.5, \mathrm{CHCl}_{3}\right) ;{ }^{1} \mathrm{H} \mathrm{NMR}\left(400 \mathrm{MHz}, \mathrm{CDCl}_{3}\right) \delta=$ 
$8.04(\mathrm{~d}, J=7.7 \mathrm{~Hz}, 2 \mathrm{H}), 7.55(\mathrm{t}, J=7.4 \mathrm{~Hz}, 1 \mathrm{H}), 7.45(\mathrm{t}, J=7.6 \mathrm{~Hz}, 2 \mathrm{H}), 5.85(\mathrm{~s}, 1$

H), 5.27-5.20 (m, $1 \mathrm{H}), 4.10-4.01(\mathrm{~m}, 1 \mathrm{H}), 3.90(\mathrm{~s}, 3 \mathrm{H}), 3.70(\mathrm{~s}, 1 \mathrm{H}), 3.21(\mathrm{~s}, 1 \mathrm{H})$, $2.20(\mathrm{dd}, J=13.3,3.0 \mathrm{~Hz}, 2 \mathrm{H}), 2.14(\mathrm{~s}, 3 \mathrm{H}), 2.06-1.83(\mathrm{~m}, 5 \mathrm{H}), 1.67$ (dd, $J=11.9$, $4.3 \mathrm{~Hz}, 1 \mathrm{H}), 1.60(\mathrm{dd}, J=14.2,2.9 \mathrm{~Hz}, 1 \mathrm{H}), 1.55-1.41(\mathrm{~m}, 2 \mathrm{H}), 1.38-1.31(\mathrm{~m}, 1 \mathrm{H})$, $1.10(\mathrm{~s}, 3 \mathrm{H}), 0.88 \mathrm{ppm}(\mathrm{d}, J=6.7 \mathrm{~Hz}, 3 \mathrm{H}) ;{ }^{13} \mathrm{C} \mathrm{NMR}\left(100 \mathrm{MHz}, \mathrm{CDCl}_{3}\right) \delta=208.6$, 174.8, 166.3, 132.8, 131.8, 130.8, 129.6 (2C), 128.4 (2C), 116.2, 75.4, 67.9, 65.2, 64.1, 63.6, 60.9, 48.3, 39.2, 35.7, 34.8, 33.9, 33.1, 28.7, 26.2, 19.9, 16.0, 12.3 ppm; HRMS (ESI): calcd for $\mathrm{C}_{28} \mathrm{H}_{34} \mathrm{NaO}_{6}{ }^{+}[\mathrm{M}+\mathrm{Na}]^{+}$489.2248, found 489.2261.

\section{Preparation of enone 23:}

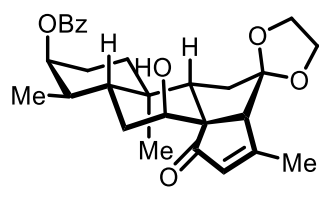

22

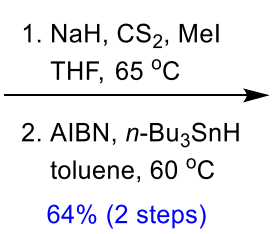

$64 \%$ (2 steps)

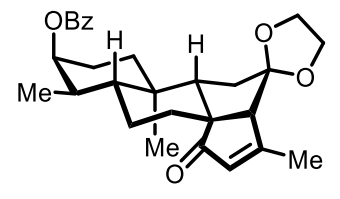

23

To a stirred solution of tetracycle $22\left(6.5 \mathrm{~g}, 14 \mathrm{mmol}, 1.0\right.$ equiv) and $\mathrm{CS}_{2}(8.4 \mathrm{~mL}$, 0.14 mol, 10 equiv) in THF $(100 \mathrm{~mL})$ at $0{ }^{\circ} \mathrm{C}$ was added $\mathrm{NaH}(60 \%$ dispersion in mineral oil, $1.7 \mathrm{~g}, 42 \mathrm{mmol}, 3.0$ equiv). The resulting mixture was warmed to $65^{\circ} \mathrm{C}$ and stirred for $5 \mathrm{~h}$ before cooled to room temperature and MeI ( $4.4 \mathrm{~mL}, 70 \mathrm{mmol}, 5.0$ equiv) was added. The resulting mixture was stirred at room temperature for $10 \mathrm{~min}$ before quenched with $\mathrm{NH}_{4} \mathrm{Cl}$ (50 mL, sat. aq.). The layers were separated, and the aqueous layer was extracted with EtOAc $(3 \times 50 \mathrm{~mL})$. The combined organic layers were dried $\left(\mathrm{Na}_{2} \mathrm{SO}_{4}\right)$ and concentrated in vacuo to afford the crude xanthate as a yellow oil, which was used directly without further purification.

To a stirred solution of xanthate (crude, obtained above) in toluene $(100 \mathrm{~mL}$, degassed) at room temperature were sequentially added AIBN (0.46 g, 2.8 mmol, 0.2 
equiv) and $n$-Bu $\mathrm{Bu}_{3} \mathrm{SnH}$ (11 mL, $42 \mathrm{mmol}, 3.0$ equiv). The resulting mixture was warmed to $60{ }^{\circ} \mathrm{C}$ and stirred for $2 \mathrm{~h}$ before cooled to room temperature and concentrated in vасио. Flash column chromatography (silica gel, hexanes:EtOAc 8:1) afforded enone 23 (4.0 g, 64\% over two steps) as a white solid. 23: m.p. $167-169{ }^{\circ} \mathrm{C}$ (hexanes/EtOAc); $R_{\mathrm{f}}=0.45$ (silica gel, hexanes:EtOAc $\left.3: 1\right) ;[\alpha]_{\mathrm{D}}{ }^{20}=-1.8\left(\mathrm{c}=0.5, \mathrm{CHCl}_{3}\right) ;{ }^{1} \mathrm{H} \mathrm{NMR}(400$ $\left.\mathrm{MHz}, \mathrm{CDCl}_{3}\right) \delta=8.04(\mathrm{~d}, J=8.0 \mathrm{~Hz}, 2 \mathrm{H}), 7.54(\mathrm{t}, J=7.3 \mathrm{~Hz}, 1 \mathrm{H}), 7.45(\mathrm{t}, J=7.1$ Hz, 2 H), 5.77 (s, $1 \mathrm{H}), 5.20(\mathrm{~s}, 1 \mathrm{H}), 4.01(\mathrm{~s}, 1 \mathrm{H}), 3.87(\mathrm{~s}, 3 \mathrm{H}), 2.63(\mathrm{~s}, 1 \mathrm{H}), 2.10$ (s, $3 \mathrm{H}), 1.98-1.76(\mathrm{~m}, 5 \mathrm{H}), 1.69$ (ddd, $J=21.8,15.6,3.9 \mathrm{~Hz}, 2 \mathrm{H}), 1.59-1.43$ (m, $4 \mathrm{H})$, 1.42-1.30 (m, $2 \mathrm{H}), 1.13(\mathrm{~s}, 3 \mathrm{H}), 0.89 \mathrm{ppm}(\mathrm{d}, J=6.6 \mathrm{~Hz}, 3 \mathrm{H}) ;{ }^{13} \mathrm{C} \mathrm{NMR}(100 \mathrm{MHz}$, $\left.\mathrm{CDCl}_{3}\right) \delta=210.0,173.0,166.2,132.8,131.3,131.0,129.6(2 \mathrm{C}), 128.4$ (2C), 116.9, $75.5,66.8,65.2,64.0,58.7,55.4,47.3,36.0,35.7,35.0,34.6,33.6,26.3,20.7,19.9$, 16.1, 12.5 ppm; HRMS (ESI): calcd for $\mathrm{C}_{28} \mathrm{H}_{34} \mathrm{NaO}_{5}{ }^{+}[\mathrm{M}+\mathrm{Na}]^{+} 473.2298$, found 473.2305 .

\section{Preparation of diol 24:}

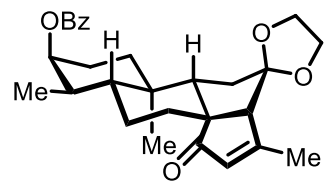

23

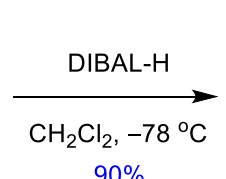

$90 \%$

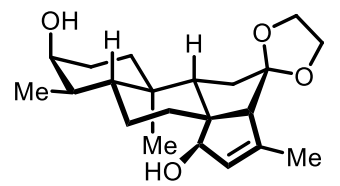

24

To a stirred solution of enone 23 (3.3 g, 7.4 mmol, 1.0 equiv) in $\mathrm{CH}_{2} \mathrm{Cl}_{2}(100 \mathrm{~mL})$ at $-78{ }^{\circ} \mathrm{C}$ was added DIBAL-H (1.5 $\mathrm{M}$ in toluene, $17 \mathrm{~mL}, 26 \mathrm{~mol}, 3.5$ equiv). The resulting mixture was stirred at $-78{ }^{\circ} \mathrm{C}$ for 30 min before diluted with EtOAc $(10 \mathrm{~mL})$ and quenched with Rochelle's salt (50 mL, sat. aq.). The layers were separated, and the aqueous layer was extracted with $\mathrm{Et}_{2} \mathrm{O}(3 \times 50 \mathrm{~mL})$. The combined organic layers were dried $\left(\mathrm{Na}_{2} \mathrm{SO}_{4}\right)$ and concentrated in vacuo. Flash column chromatography (silica gel, 
hexanes:EtOAc 3:1) afforded diol 24 (2.3 g, 90\%) as a white solid. 24: m.p. 171-172 ${ }^{\circ} \mathrm{C}\left(\mathrm{CH}_{2} \mathrm{Cl}_{2}\right) ; R_{\mathrm{f}}=0.50$ (silica gel, hexanes:EtOAc 1:1); $[\alpha]_{\mathrm{D}}{ }^{20}=+1.6\left(\mathrm{c}=0.5, \mathrm{CHCl}_{3}\right)$; ${ }^{1} \mathrm{H}$ NMR $\left(400 \mathrm{MHz}, \mathrm{CDCl}_{3}\right) \delta=5.35(\mathrm{~s}, 1 \mathrm{H}), 4.83(\mathrm{~d}, J=6.5 \mathrm{~Hz}, 1 \mathrm{H}), 3.94(\mathrm{dd}, J=$ 6.4, 3.7 Hz, $1 \mathrm{H}), 3.88-3.78$ (m, $3 \mathrm{H}), 3.77$ (s, $1 \mathrm{H}), 2.34$ (s, $1 \mathrm{H}), 2.01-1.94$ (m, $1 \mathrm{H})$, $1.84(\mathrm{ddd}, J=17.7,8.7,3.7 \mathrm{~Hz}, 1 \mathrm{H}), 1.74(\mathrm{~s}, 3 \mathrm{H}), 1.70-1.63(\mathrm{~m}, 3 \mathrm{H}), 1.59(\mathrm{dd}, J=$ 13.1, $3.5 \mathrm{~Hz}, 1 \mathrm{H}), 1.56-1.45$ (m, $4 \mathrm{H}), 1.44-1.29$ (m, $3 \mathrm{H}), 1.27-1.16$ (m, $2 \mathrm{H}), 0.94$ (s, $3 \mathrm{H}), 0.90 \mathrm{ppm}(\mathrm{d}, J=6.8 \mathrm{~Hz}, 3 \mathrm{H}) ;{ }^{13} \mathrm{C} \mathrm{NMR}\left(100 \mathrm{MHz}, \mathrm{CDCl}_{3}\right) \delta=140.9,130.0$, $118.2,77.9,72.3,71.2,65.2,64.0,57.7,55.2,45.5,35.9,35.3,34.8,34.6,33.1,28.8$, 23.9, 17.3, 16.4, 10.9 ppm; HRMS (ESI): calcd for $\mathrm{C}_{21} \mathrm{H}_{32} \mathrm{NaO}_{4}{ }^{+}[\mathrm{M}+\mathrm{Na}]^{+}$371.2193, found 371.2201 .

\section{Preparation of epoxide 25:}

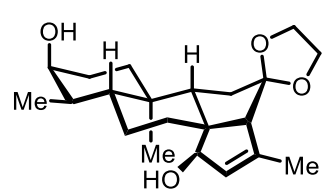

24

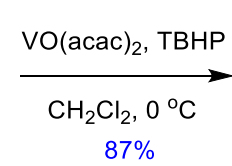

$87 \%$

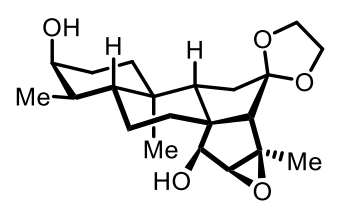

25

To a stirred solution of diol 24 (1.6 g, $4.5 \mathrm{mmol}, 1.0$ equiv) in $\mathrm{CH}_{2} \mathrm{Cl}_{2}(50 \mathrm{~mL})$ at $0{ }^{\circ} \mathrm{C}$ were sequentially added $4 \AA ̊$ molecular sieves $(1.5 \mathrm{~g}), \mathrm{VO}(\mathrm{acac})_{2}(0.29 \mathrm{~g}, 1.1 \mathrm{mmol}$, 0.2 equiv) and TBHP (5.5 $\mathrm{M}$ in decane, $2.5 \mathrm{~mL}, 14 \mathrm{mmol}, 3.0$ equiv). The resulting mixture was stirred at $0{ }^{\circ} \mathrm{C}$ for $6 \mathrm{~h}$ before quenched with $\mathrm{Na}_{2} \mathrm{~S}_{2} \mathrm{O}_{3}(30 \mathrm{~mL}$, sat. aq.). The layers were separated, and the aqueous layer was extracted with $\mathrm{CH}_{2} \mathrm{Cl}_{2}(3 \times 30 \mathrm{~mL})$. The combined organic layers were dried $\left(\mathrm{Na}_{2} \mathrm{SO}_{4}\right)$ and concentrated in vacuo. Flash column chromatography (silica gel, hexanes:EtOAc 4:1) afforded epoxide 25 (1.4 g, 87\%) as a white solid. 25: m.p. $180-182{ }^{\circ} \mathrm{C}$ (hexanes/EtOAc); $R_{\mathrm{f}}=0.45$ (silica gel, hexanes:EtOAc 1:1); $[\alpha]_{\mathrm{D}}{ }^{20}=-2.0\left(\mathrm{c}=0.5, \mathrm{CHCl}_{3}\right) ;{ }^{1} \mathrm{H} \mathrm{NMR}\left(400 \mathrm{MHz}, \mathrm{CDCl}_{3}\right) \delta=$ 
$4.29(\mathrm{~d}, J=10.7 \mathrm{~Hz}, 1 \mathrm{H}), 4.02-3.94(\mathrm{~m}, 1 \mathrm{H}), 3.92-3.85(\mathrm{~m}, 1 \mathrm{H}), 3.84-3.76(\mathrm{~m}, 2 \mathrm{H})$, $3.74(\mathrm{~s}, 1 \mathrm{H}), 3.47(\mathrm{~s}, 1 \mathrm{H}), 2.21(\mathrm{~d}, J=17.3 \mathrm{~Hz}, 2 \mathrm{H}), 2.13(\mathrm{~s}, 1 \mathrm{H}), 1.80(\mathrm{t}, J=14.2$ $\mathrm{Hz}, 1 \mathrm{H}), 1.73(\mathrm{~d}, J=6.9 \mathrm{~Hz}, 1 \mathrm{H}), 1.65(\mathrm{t}, J=13.8 \mathrm{~Hz}, 1 \mathrm{H}), 1.60-1.45(\mathrm{~m}, 4 \mathrm{H}), 1.42$ $(\mathrm{d}, J=8.1 \mathrm{~Hz}, 6 \mathrm{H}), 1.30-1.21(\mathrm{~m}, 2 \mathrm{H}), 1.16(\mathrm{~d}, J=11.6 \mathrm{~Hz}, 1 \mathrm{H}), 0.92(\mathrm{~s}, 3 \mathrm{H}), 0.87$ $\operatorname{ppm}(\mathrm{d}, J=6.8 \mathrm{~Hz}, 3 \mathrm{H}) ;{ }^{13} \mathrm{C} \mathrm{NMR}\left(100 \mathrm{MHz}, \mathrm{CDCl}_{3}\right) \delta=116.3,73.4,72.1,68.5,66.1$, $64.5,63.5,62.3,54.8,50.2,45.2,37.0,35.9,35.6,34.7,33.1,28.8,23.9,17.4,16.2$ 10.2 ppm; HRMS (ESI): calcd for $\mathrm{C}_{21} \mathrm{H}_{32} \mathrm{NaO}_{5}{ }^{+}[\mathrm{M}+\mathrm{Na}]^{+}$387.2142, found 387.2149.

\section{Preparation of cyclohexadienone 26:}

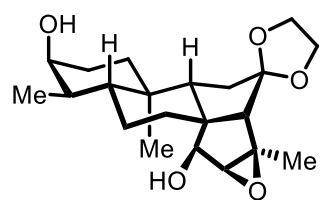

25

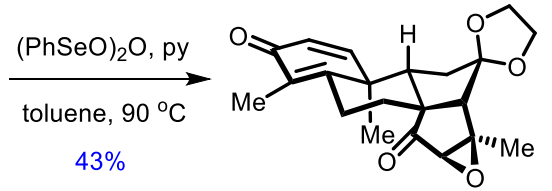

26

To a stirred solution of epoxide 25 ( $1.2 \mathrm{~g}, 3.3 \mathrm{~mol}, 1.0$ equiv) in toluene (50 mL) at room temperature were sequentially added pyridine $(1.3 \mathrm{~mL}, 17 \mathrm{mmol}, 5.0$ equiv) and $(\mathrm{PhSeO})_{2} \mathrm{O}\left(5.9 \mathrm{~g}, 17 \mathrm{~mol}, 5.0\right.$ equiv). The resulting mixture was warmed to $90{ }^{\circ} \mathrm{C}$ and stirred for $12 \mathrm{~h}$ before cooled to room temperature and concentrated in vacuo. Flash column chromatography (silica gel, hexanes:EtOAc 6:1) afforded cyclohexadienone 26 $(0.51 \mathrm{~g}, 43 \%)$ as a white solid. 26: m.p. $187-189^{\circ} \mathrm{C}($ hexanes $/ \mathrm{EtOAc}) ; R_{\mathrm{f}}=0.45($ silica gel, hexanes:EtOAc 2:1); $[\alpha]_{\mathrm{D}}{ }^{20}=-10.8\left(\mathrm{c}=0.5, \mathrm{CHCl}_{3}\right) ;{ }^{1} \mathrm{H} \mathrm{NMR}\left(400 \mathrm{MHz}, \mathrm{CDCl}_{3}\right)$ $\delta=6.82(\mathrm{~d}, J=9.9 \mathrm{~Hz}, 1 \mathrm{H}), 6.15(\mathrm{~d}, J=9.9 \mathrm{~Hz}, 1 \mathrm{H}), 4.04-3.97(\mathrm{~m}, 1 \mathrm{H}), 3.92(\mathrm{td}, J$ = 7.0, $4.6 \mathrm{~Hz}, 1 \mathrm{H}), 3.86(\mathrm{q}, J=7.1 \mathrm{~Hz}, 1 \mathrm{H}), 3.79(\mathrm{q}, J=7.0 \mathrm{~Hz}, 1 \mathrm{H}), 3.32(\mathrm{~s}, 1 \mathrm{H})$, 2.73-2.63 (m, $1 \mathrm{H}), 2.49$ (s, $1 \mathrm{H}), 2.47-2.41(\mathrm{~m}, 1 \mathrm{H}), 2.35$ (dd, $J=14.0,3.1 \mathrm{~Hz}, 1 \mathrm{H})$, $1.88(\mathrm{~s}, 3 \mathrm{H}), 1.83(\mathrm{~d}, J=25.9 \mathrm{~Hz}, 1 \mathrm{H}), 1.58(\mathrm{~d}, J=6.6 \mathrm{~Hz}, 5 \mathrm{H}), 1.44(\mathrm{~s}, 3 \mathrm{H}), 1.44$ $1.38 \mathrm{ppm}(\mathrm{m}, 1 \mathrm{H}) ;{ }^{13} \mathrm{C} \mathrm{NMR}\left(100 \mathrm{MHz}, \mathrm{CDCl}_{3}\right) \delta=211.0,185.8,160.5,154.9,129.8$, 
$125.8,114.9,64.9,63.7,63.4,62.8,61.2,58.2,50.6,42.6,37.5,36.9,25.2,21.7,17.3$, 10.8 ppm; HRMS (ESI): calcd for $\mathrm{C}_{21} \mathrm{H}_{24} \mathrm{NaO}_{5}{ }^{+}[\mathrm{M}+\mathrm{Na}]^{+} 379.1516$, found 379.1526.

\section{Preparation of alcohols 27 and C14-epi-27:}

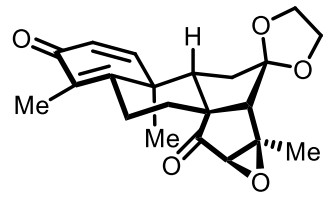

26

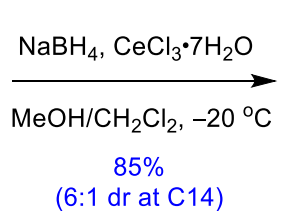

$(6: 1 \mathrm{dr}$ at $\mathrm{C} 14)$

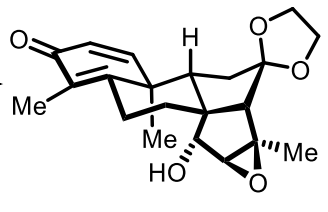

27

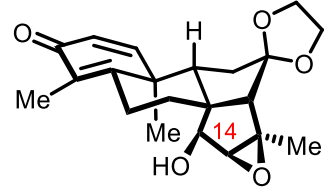

C14-epi-27

To a stirred solution of cyclohexadienone 26 ( $0.61 \mathrm{~g}, 1.7 \mathrm{mmol}, 1.0$ equiv) in $\mathrm{MeOH} / \mathrm{CH}_{2} \mathrm{Cl}_{2}(1: 1,40 \mathrm{~mL})$ at $-20{ }^{\circ} \mathrm{C}$ were sequentially added $\mathrm{CeCl}_{3} \cdot 7 \mathrm{H}_{2} \mathrm{O}(1.3 \mathrm{~g}, 3.4$ mmol, 2.0 equiv) and $\mathrm{NaBH}_{4}(96 \mathrm{mg}, 2.6 \mathrm{mmol}, 1.5$ equiv). The resulting mixture was stirred at $-20{ }^{\circ} \mathrm{C}$ for $15 \mathrm{~min}$ before quenched with acetone $(5 \mathrm{~mL})$ and $\mathrm{H}_{2} \mathrm{O}(20 \mathrm{~mL})$. The layers were separated, and the aqueous layer was extracted with $\mathrm{CH}_{2} \mathrm{Cl}_{2}(3 \times 20$ $\mathrm{mL})$. The combined organic layers were dried $\left(\mathrm{Na}_{2} \mathrm{SO}_{4}\right)$ and concentrated in vacuo. Flash column chromatography (silica gel, hexanes:EtOAc 2:1) afforded alcohol 27 (0.44 g, 73\%) and C14-epi-27 (73 mg, 12\%) as white solids. 27: m.p. $163-165{ }^{\circ} \mathrm{C}$ (hexanes/EtOAc); $R_{\mathrm{f}}=0.30$ (silica gel, hexanes:EtOAc $\left.1: 1\right) ;[\alpha]_{\mathrm{D}}^{20}=-3.4(\mathrm{c}=0.5$, $\left.\mathrm{CHCl}_{3}\right) ;{ }^{1} \mathrm{H} \mathrm{NMR}\left(400 \mathrm{MHz}, \mathrm{CDCl}_{3}\right) \delta=6.72(\mathrm{~d}, J=9.9 \mathrm{~Hz}, 1 \mathrm{H}), 6.26-6.11(\mathrm{~m}, 1 \mathrm{H})$, $4.25(\mathrm{~d}, J=11.6 \mathrm{~Hz}, 1 \mathrm{H}), 4.11-4.03(\mathrm{~m}, 1 \mathrm{H}), 3.98(\mathrm{t}, J=6.4 \mathrm{~Hz}, 2 \mathrm{H}), 3.87$ (q, $J=7.4$ Hz, $1 \mathrm{H}), 3.42(\mathrm{~s}, 1 \mathrm{H}), 3.07(\mathrm{dd}, J=11.6,2.1 \mathrm{~Hz}, 1 \mathrm{H}), 2.72(\mathrm{~d}, J=16.0 \mathrm{~Hz}, 1 \mathrm{H}), 2.43$ $(\mathrm{t}, J=12.7 \mathrm{~Hz}, 1 \mathrm{H}), 2.32-2.23(\mathrm{~m}, 1 \mathrm{H}), 2.18(\mathrm{~d}, J=15.1 \mathrm{~Hz}, 2 \mathrm{H}), 1.96-1.79(\mathrm{~m}, 2$ H), 1.89 (s, $3 \mathrm{H}), 1.45$ (s, $3 \mathrm{H}), 1.29$ (s, $3 \mathrm{H}), 1.18 \mathrm{ppm}(\mathrm{t}, J=13.6 \mathrm{~Hz}, 1 \mathrm{H}) ;{ }^{13} \mathrm{C} \mathrm{NMR}$ $\left(100 \mathrm{MHz}, \mathrm{CDCl}_{3}\right) \delta=186.0,161.3,154.7,128.9,126.6,117.0,76.0,64.9,64.1,64.0$ 63.1, 61.0, 54.2, 52.4, 43.3, 40.5, 38.8, 25.0, 21.6, 16.5, 10.8 ppm; HRMS (ESI): calcd for $\mathrm{C}_{21} \mathrm{H}_{26} \mathrm{NaO}_{5}{ }^{+}[\mathrm{M}+\mathrm{Na}]^{+}$381.1672, found 381.1679. 
C14-epi-27: m.p. $155-157^{\circ} \mathrm{C}$ (hexanes/EtOAc); $R_{\mathrm{f}}=0.25$ (silica gel, hexanes:EtOAc $1: 1) ;[\alpha]_{\mathrm{D}}{ }^{20}=-32.8\left(\mathrm{c}=0.5, \mathrm{CHCl}_{3}\right) ;{ }^{1} \mathrm{H} \mathrm{NMR}\left(400 \mathrm{MHz}, \mathrm{CDCl}_{3}\right) \delta=6.77(\mathrm{~d}, J=9.9$ $\mathrm{Hz}, 1 \mathrm{H}), 6.20(\mathrm{~d}, J=9.9 \mathrm{~Hz}, 1 \mathrm{H}), 4.49(\mathrm{dd}, J=10.1,1.3 \mathrm{~Hz}, 1 \mathrm{H}), 4.03-3.90(\mathrm{~m}, 2$ H), 3.87-3.81 (m, $1 \mathrm{H}), 3.81-3.75(\mathrm{~m}, 1 \mathrm{H}), 3.55$ (s, $1 \mathrm{H}), 2.95-2.82(\mathrm{~m}, 1 \mathrm{H}), 2.70$ (ddd, $J=14.6,4.6,2.5 \mathrm{~Hz}, 1 \mathrm{H}), 2.43(\mathrm{ddd}, J=13.7,5.1,2.5 \mathrm{~Hz}, 1 \mathrm{H}), 2.26(\mathrm{dd}, J=$ 10.3, 2.5 Hz, $1 \mathrm{H}), 2.22(\mathrm{~s}, 1 \mathrm{H}), 1.89(\mathrm{~d}, J=1.1 \mathrm{~Hz}, 3 \mathrm{H}), 1.86(\mathrm{~s}, 1 \mathrm{H}), 1.69-1.56(\mathrm{~m}$, $2 \mathrm{H}), 1.46$ (s, $3 \mathrm{H}), 1.33$ (s, $3 \mathrm{H}), 1.29 \mathrm{ppm}(\mathrm{dd}, J=13.7,4.6 \mathrm{~Hz}, 1 \mathrm{H}) .{ }^{13} \mathrm{C}$ NMR $(100$ $\left.\mathrm{MHz}, \mathrm{CDCl}_{3}\right) \delta=186.0,161.8,154.9,129.1,126.6,115.7,73.4,68.1,64.8(2 \mathrm{C}), 63.7$, 62.3, 50.6, 50.4, 43.0, 37.0, 36.9, 27.1, 18.2, 17.3, 10.9 ppm; HRMS (ESI): calcd for $\mathrm{C}_{21} \mathrm{H}_{26} \mathrm{NaO}_{5}^{+}[\mathrm{M}+\mathrm{Na}]^{+}$381.1672, found 381.1683.

\section{Preparation of spirocycle 30:}

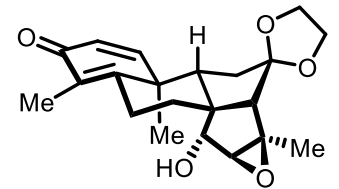

27

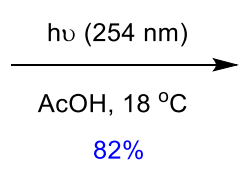

$82 \%$

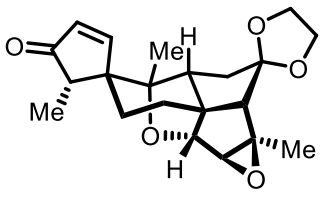

30

A stirred solution of alcohol $27(10 \mathrm{mg}, 0.028 \mathrm{mmol})$ in $\mathrm{AcOH}(3 \mathrm{~mL})$ at $18{ }^{\circ} \mathrm{C}$ was irradiated with a $254 \mathrm{~nm}$ lamp $(300 \mathrm{~W})$ for $20 \mathrm{~min}$ before concentrated in vacuo. The residue was partitioned between $\mathrm{CH}_{2} \mathrm{Cl}_{2}(5 \mathrm{~mL})$ and $\mathrm{NaHCO}_{3}(5 \mathrm{~mL}$, sat. aq.). The layers were separated, and the aqueous layer was extracted with $\mathrm{CH}_{2} \mathrm{Cl}_{2}(3 \times 5 \mathrm{~mL})$. The combined organic layers were dried $\left(\mathrm{Na}_{2} \mathrm{SO}_{4}\right)$ and concentrated in vacuo. Flash column chromatography (silica gel, hexanes:EtOAc 4:1) afforded spirocycle 30 (8.2 mg, $82 \%$ ) as a white solid. 30: m.p. $130-132{ }^{\circ} \mathrm{C}\left(\mathrm{CH}_{2} \mathrm{Cl}_{2} / \mathrm{Et}_{2} \mathrm{O}\right) ; R_{\mathrm{f}}=0.40$ (silica gel, hexanes:EtOAc 2:1); $[\alpha]_{\mathrm{D}}{ }^{20}=-22.9\left(\mathrm{c}=0.5, \mathrm{CHCl}_{3}\right) ;{ }^{1} \mathrm{H} \mathrm{NMR}\left(400 \mathrm{MHz}, \mathrm{CDCl}_{3}\right) \delta=$ $7.50(\mathrm{~d}, J=6.0 \mathrm{~Hz}, 1 \mathrm{H}), 6.17(\mathrm{~d}, J=6.0 \mathrm{~Hz}, 1 \mathrm{H}), 4.40(\mathrm{~s}, 1 \mathrm{H}), 4.01-3.95(\mathrm{~m}, 2 \mathrm{H})$, 
3.94-3.87 (m, $2 \mathrm{H}), 3.23(\mathrm{~s}, 1 \mathrm{H}), 2.26-2.16(\mathrm{~m}, 1 \mathrm{H}), 2.15(\mathrm{~d}, J=1.4 \mathrm{~Hz}, 1 \mathrm{H}), 2.14$ 2.04 (m, 2 H), 2.04-1.94 (m, 1 H), 1.80-1.71 (m, 3 H), 1.57 (ddd, $J=11.9,6.2,2.0 \mathrm{~Hz}$, $1 \mathrm{H}), 1.51(\mathrm{~s}, 3 \mathrm{H}), 1.35$ (d, $J=7.4 \mathrm{~Hz}, 3 \mathrm{H}), 0.91 \mathrm{ppm}(\mathrm{s}, 3 \mathrm{H}) ;{ }^{13} \mathrm{C} \mathrm{NMR}(100 \mathrm{MHz}$, $\left.\mathrm{CDCl}_{3}\right) \delta=209.6,166.3,132.3,116.8,89.0,85.5,71.2,65.6,64.2,64.1,63.2,57.0$ 56.2, 55.7, 54.9, 33.8, 33.4, 33.3, 19.3, 18.0, 9.6 ppm; HRMS (ESI): calcd for $\mathrm{C}_{21} \mathrm{H}_{26} \mathrm{O}_{5}{ }^{+}[\mathrm{M}+\mathrm{H}]^{+}$359.1853, found 359.1859.

\section{Photo-Nazarov Cyclization of Cyclohexadienone 28}

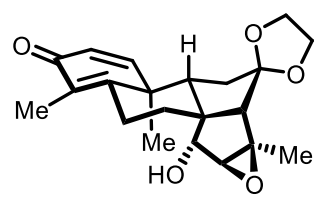

27

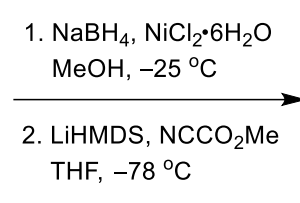

$72 \%$ (2 steps)

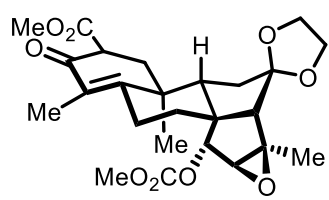

S-7

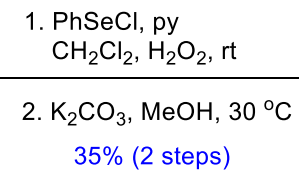

$35 \%$ (2 steps)

(a)

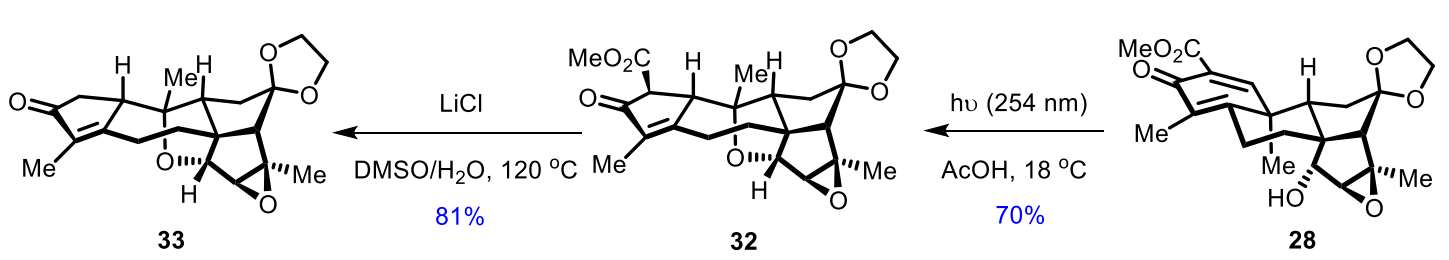

Scheme S3. Photo-Nazarov cyclization of cyclohexadienone 28

\section{Preparation of ester S-7:}

To a stirred solution of dienone 27 (50 mg, $0.14 \mathrm{mmol}, 1.0$ equiv) in $\mathrm{MeOH}$ (5 mL) at $-25{ }^{\circ} \mathrm{C}$ were sequentially added $\mathrm{NiCl}_{2} \cdot 6 \mathrm{H}_{2} \mathrm{O}(50 \mathrm{mg}, 0.21 \mathrm{mmol}, 1.5$ equiv $)$ and $\mathrm{NaBH}_{4}\left(7.9 \mathrm{mg}, 0.21 \mathrm{mmol}, 1.5\right.$ equiv). The resulting mixture was stirred at $-25^{\circ} \mathrm{C}$ for 30 min before diluted with $\mathrm{CH}_{2} \mathrm{Cl}_{2}(5 \mathrm{~mL})$, and quenched with acetone (1 mL) and $\mathrm{H}_{2} \mathrm{O}$ ( $5 \mathrm{~mL}$ ). The layers were separated, and the aqueous layer was extracted with $\mathrm{CH}_{2} \mathrm{Cl}_{2}$ (3 $\times 5 \mathrm{~mL})$. The combined organic layers were dried $\left(\mathrm{Na}_{2} \mathrm{SO}_{4}\right)$ and concentrated in vacuo. 
Flash column chromatography (silica gel, hexanes:EtOAc 2:1) afforded the corresponding enone as a white solid.

To a stirred solution of enone (obtained above) in THF $(5 \mathrm{~mL})$ at $-78{ }^{\circ} \mathrm{C}$ were sequentially added LiHMDS (1.0 $\mathrm{M}$ in THF, $0.38 \mathrm{~mL}, 0.38 \mathrm{mmol}, 2.7$ equiv) and $\mathrm{NCCO}_{2} \mathrm{Me}\left(30 \mu \mathrm{L}, 0.35 \mathrm{mmol}, 2.5\right.$ equiv). The resulting mixture was stirred at $-78{ }^{\circ} \mathrm{C}$ for $1 \mathrm{~h}$ before quenched with $\mathrm{NaHCO}_{3}$ ( $5 \mathrm{~mL}$, sat. aq.). The layers were separated, and the aqueous layer was extracted with EtOAc $(3 \times 5 \mathrm{~mL})$. The combined organic layers were dried $\left(\mathrm{Na}_{2} \mathrm{SO}_{4}\right)$ and concentrated in vacuo. Flash column chromatography (silica gel, hexanes:EtOAc 5:1) afforded ester $\mathbf{S - 7}$ (48 mg, 72\% over two steps) as a white solid. S-7: m.p. $168-170{ }^{\circ} \mathrm{C}$ (hexanes/EtOAc); $R_{\mathrm{f}}=0.40$ (silica gel, hexanes:EtOAc $2: 1) ;[\alpha]_{\mathrm{D}}^{20}=-27.2\left(\mathrm{c}=0.15, \mathrm{CHCl}_{3}\right) ;{ }^{1} \mathrm{H} \mathrm{NMR}\left(400 \mathrm{MHz}, \mathrm{CDCl}_{3}\right) \delta=5.14(\mathrm{~s}, 1 \mathrm{H})$, 4.04-3.95 (m, $2 \mathrm{H}), 3.88$ (q, $J=6.8 \mathrm{~Hz}, 1 \mathrm{H}), 3.81(\mathrm{~s}, 3 \mathrm{H}), 3.81-3.74(\mathrm{~m}, 1 \mathrm{H}), 3.77$ (s, $3 \mathrm{H}), 3.56(\mathrm{~s}, 1 \mathrm{H}), 3.51(\mathrm{dd}, J=14.7,4.7 \mathrm{~Hz}, 1 \mathrm{H}), 2.71-2.62(\mathrm{~m}, 1 \mathrm{H}), 2.36-2.22$ (m, $3 \mathrm{H}), 2.20-2.13(\mathrm{~m}, 1 \mathrm{H}), 2.09(\mathrm{t}, J=14.0 \mathrm{~Hz}, 1 \mathrm{H}), 1.94-1.83(\mathrm{~m}, 2 \mathrm{H}), 1.81-1.72$ $(\mathrm{m}, 4 \mathrm{H}), 1.55(\mathrm{~s}, 3 \mathrm{H}), 1.41(\mathrm{td}, J=13.7,3.5 \mathrm{~Hz}, 1 \mathrm{H}), 1.06 \mathrm{ppm}(\mathrm{s}, 3 \mathrm{H}) ;{ }^{13} \mathrm{C} \mathrm{NMR}$ $\left(100 \mathrm{MHz} \mathrm{CDCl}_{3}\right) \delta=193.2,171.6,162.2,155.3,128.4,116.3,80.8,65.0,64.3,64.2$, 64.1, 63.6, 56.0, 55.3, 52.8, 52.4, 49.8, 39.2, 38.4, 38.3, 38.2, 24.9, 18.1, 16.6, 11.4 ppm; HRMS (ESI): calcd for $\mathrm{C}_{25} \mathrm{H}_{32} \mathrm{NaO}_{9}{ }^{+}[\mathrm{M}+\mathrm{Na}]^{+}$499.1939, found 499.1946.

\section{Preparation of cyclohexadienone 28:}

To a stirred solution of ester $\mathbf{S}-7$ (48 $\mathrm{mg}, 0.10 \mathrm{mmol}, 1.0$ equiv) in $\mathrm{CH}_{2} \mathrm{Cl}_{2}(5 \mathrm{~mL})$ at room temperature were sequentially added pyridine $(11 \mu \mathrm{L}, 0.14 \mathrm{mmol}, 1.4$ equiv) and $\mathrm{PhSeCl}$ (21 mg, $0.11 \mathrm{mmol}, 1.1$ equiv). The resulting mixture was stirred at room 
temperature for $1 \mathrm{~h}$ before cooled to $0{ }^{\circ} \mathrm{C}$ and added $\mathrm{H}_{2} \mathrm{O}_{2}(30 \%$ wt/wt aq., $96 \mu \mathrm{L}, 0.95$ mmol, 9.5 equiv). The resulting mixture was stirred for $20 \mathrm{~min}$ before quenched with $\mathrm{Na}_{2} \mathrm{~S}_{2} \mathrm{O}_{3}$ (5 mL, sat. aq.). The layers were separated, and the aqueous layer was extracted with $\mathrm{CH}_{2} \mathrm{Cl}_{2}(3 \times 5 \mathrm{~mL})$. The combined organic layers were dried $\left(\mathrm{Na}_{2} \mathrm{SO}_{4}\right)$ and concentrated in vacuo to afford the crude dienone as a yellow oil, which was used directly without further purification.

To a stirred solution of dienone (crude, obtained above) in $\mathrm{MeOH}(5 \mathrm{~mL})$ at $30{ }^{\circ} \mathrm{C}$ was added $\mathrm{K}_{2} \mathrm{CO}_{3}$ (19 mg, $0.14 \mathrm{mmol}, 1.4$ equiv). The resulting mixture was stirred at $30{ }^{\circ} \mathrm{C}$ for $12 \mathrm{~h}$ before diluted with $\mathrm{CH}_{2} \mathrm{Cl}_{2}(5 \mathrm{~mL})$ and quenched with $\mathrm{NH}_{4} \mathrm{Cl}$ (5 mL, sat. aq.). The layers were separated, and the aqueous layer was extracted with $\mathrm{CH}_{2} \mathrm{Cl}_{2}(3 \times$ $5 \mathrm{~mL})$. The combined organic layers were dried $\left(\mathrm{Na}_{2} \mathrm{SO}_{4}\right)$ and concentrated in vacuo. Flash column chromatography (silica gel, hexanes:EtOAc 2:1) afforded cyclohexadienone $\mathbf{2 8}$ (15 mg, 35\% over two steps) as a white solid. 28: m.p. 181-183 ${ }^{\circ} \mathrm{C}$ (hexanes/EtOAc); $R_{\mathrm{f}}=0.30$ (silica gel, hexanes:EtOAc 1:2); $[\alpha]_{\mathrm{D}}{ }^{20}=-1.9(\mathrm{c}=0.5$, $\left.\mathrm{CHCl}_{3}\right) ;{ }^{1} \mathrm{H} \mathrm{NMR}\left(400 \mathrm{MHz}, \mathrm{CDCl}_{3}\right) \delta=7.36(\mathrm{~s}, 1 \mathrm{H}), 4.27(\mathrm{~d}, J=11.7 \mathrm{~Hz}, 1 \mathrm{H}), 4.13-$ $4.06(\mathrm{~m}, 1 \mathrm{H}), 4.04-3.97$ (m, $2 \mathrm{H}), 3.90(\mathrm{q}, J=7.4 \mathrm{~Hz}, 1 \mathrm{H}), 3.84(\mathrm{~s}, 3 \mathrm{H}), 3.44(\mathrm{~s}, 1 \mathrm{H})$, $3.04(\mathrm{~d}, J=11.8 \mathrm{~Hz}, 1 \mathrm{H}), 2.79-2.67(\mathrm{~m}, 1 \mathrm{H}), 2.45(\mathrm{td}, J=14.3,4.0 \mathrm{~Hz}, 1 \mathrm{H}), 2.33-$ $2.25(\mathrm{~m}, 1 \mathrm{H}), 2.21(\mathrm{~d}, J=16.2 \mathrm{~Hz}, 2 \mathrm{H}), 1.99(\mathrm{dd}, J=12.4,8.9 \mathrm{~Hz}, 1 \mathrm{H}), 1.92(\mathrm{~s}, 4 \mathrm{H})$, 1.47 (s, $3 \mathrm{H}), 1.36$ (s, $3 \mathrm{H}), 1.21 \mathrm{ppm}(\mathrm{td}, J=13.7,3.7 \mathrm{~Hz}, 1 \mathrm{H}) ;{ }^{13} \mathrm{C} \mathrm{NMR}(100 \mathrm{MHz}$, $\left.\mathrm{CDCl}_{3}\right) \delta=181.5,166.4,159.8,158.7,129.8,129.7,116.9,76.1,64.8,64.2,64.1,63.1$, 61.0, 54.4, 52.5, 51.9, 43.1, 40.4, 38.7, 25.0, 21.3, 16.5, 11.1 ppm; HRMS (ESI): calcd for $\mathrm{C}_{23} \mathrm{H}_{28} \mathrm{NaO}_{7}{ }^{+}[\mathrm{M}+\mathrm{Na}]^{+}$439.1727, found 439.1736. 


\section{Preparation of pentacyclic ester 32:}

A stirred solution of cyclohexadienone $28(12 \mathrm{mg}, 29 \mu \mathrm{mol})$ in $\mathrm{AcOH}(3 \mathrm{~mL})$ at $18{ }^{\circ} \mathrm{C}$ was irradiated with a $254 \mathrm{~nm}$ mercury lamp (300 W) for $20 \mathrm{~min}$ before concentrated in vacuo. The residue was partitioned between $\mathrm{CH}_{2} \mathrm{Cl}_{2}(5 \mathrm{~mL})$ and $\mathrm{NaHCO}_{3}(5 \mathrm{~mL}$, sat. aq.). The layers were separated, and the aqueous layer was extracted with $\mathrm{CH}_{2} \mathrm{Cl}_{2}(3 \times 5 \mathrm{~mL})$. The combined organic layers were dried $\left(\mathrm{Na}_{2} \mathrm{SO}_{4}\right)$ and concentrated in vacuo. Flash column chromatography (silica gel, hexanes:EtOAc 4:1) afforded pentacyclic ester 32 (8.4 mg, 70\%) as a white solid. 32: m.p. $203-205^{\circ} \mathrm{C}$ (hexanes/EtOAc); $R_{\mathrm{f}}=0.60$ (silica gel, hexanes:EtOAc 1:2); $[\alpha]_{\mathrm{D}}{ }^{20}=-27.9(\mathrm{c}=0.5$, $\left.\mathrm{CHCl}_{3}\right) ;{ }^{1} \mathrm{H} \mathrm{NMR}\left(400 \mathrm{MHz}, \mathrm{CDCl}_{3}\right) \delta=4.01-3.89(\mathrm{~m}, 4 \mathrm{H}), 3.75(\mathrm{~s}, 3 \mathrm{H}), 3.65(\mathrm{~s}, 2$ H), $3.19(\mathrm{~s}, 1 \mathrm{H}), 3.06(\mathrm{~s}, 1 \mathrm{H}), 2.86(\mathrm{dd}, J=13.0,6.8 \mathrm{~Hz}, 1 \mathrm{H}), 2.68(\mathrm{dd}, J=11.0,7.9$ $\mathrm{Hz}, 1 \mathrm{H}), 2.49(\mathrm{q}, J=12.1 \mathrm{~Hz}, 1 \mathrm{H}), 2.08-1.99(\mathrm{~m}, 2 \mathrm{H}), 1.82(\mathrm{ddd}, J=25.2,11.8,6.0$ $\mathrm{Hz}, 3 \mathrm{H}), 1.70$ (s, $3 \mathrm{H}), 1.46$ (s, $3 \mathrm{H}), 1.20 \mathrm{ppm}(\mathrm{s}, 3 \mathrm{H}) ;{ }^{13} \mathrm{C} \mathrm{NMR}\left(100 \mathrm{MHz}, \mathrm{CDCl}_{3}\right)$ $\delta=200.8,172.7,170.4,136.7,115.5,90.0,86.8,68.4,67.3,65.6,65.4,63.3,61.4,57.5$, 53.7, 52.9, 49.5, 37.0, 35.1, 27.2, 22.3, 17.1, 8.5 ppm; HRMS (ESI): calcd for $\mathrm{C}_{23} \mathrm{H}_{28} \mathrm{NaO}_{7}^{+}[\mathrm{M}+\mathrm{Na}]^{+}$439.1727, found 439.1732.

\section{Preparation of pentacycle 33:}

To a stirred solution of pentacyclic ester $\mathbf{3 2}(8.3 \mathrm{mg}, 19 \mu \mathrm{mol}, 1.0$ equiv) in DMSO (2 $\mathrm{mL})$ at room temperature was added $\mathrm{LiCl}(8.1 \mathrm{mg}, 0.19 \mathrm{mmol}, 10$ equiv). The resulting mixture was warmed to $120{ }^{\circ} \mathrm{C}$ and stirred for $16 \mathrm{~h}$ before cooled to room temperature, diluted with $\mathrm{Et}_{2} \mathrm{O}(5 \mathrm{~mL})$ and quenched with $\mathrm{H}_{2} \mathrm{O}(5 \mathrm{~mL})$. The layers were separated, and the aqueous layer was extracted with $\mathrm{Et}_{2} \mathrm{O}(3 \times 5 \mathrm{~mL})$. The combined 
organic layers were dried $\left(\mathrm{Na}_{2} \mathrm{SO}_{4}\right)$ and concentrated in vacuo. Flash column chromatography (silica gel, hexanes:EtOAc 2:1) afforded pentacycle 33 (5.8 $\mathrm{mg}, 81 \%$ ) as a white solid. 33: m.p. $212-214{ }^{\circ} \mathrm{C}$ (hexanes/EtOAc); $R_{\mathrm{f}}=0.50$ (silica gel, hexanes:EtOAc 1:1); $[\alpha]_{\mathrm{D}}{ }^{20}=-17.5\left(\mathrm{c}=0.1, \mathrm{CHCl}_{3}\right) ;{ }^{1} \mathrm{H} \mathrm{NMR}\left(400 \mathrm{MHz}, \mathrm{CDCl}_{3}\right) \delta=$ 4.02-3.90 (m, $4 \mathrm{H}), 3.67(\mathrm{~s}, 1 \mathrm{H}), 3.20(\mathrm{~d}, J=1.0 \mathrm{~Hz}, 1 \mathrm{H}), 2.84(\mathrm{dd}, J=13.0,6.8 \mathrm{~Hz}$, $1 \mathrm{H}), 2.67(\mathrm{dd}, J=11.5,7.4 \mathrm{~Hz}, 1 \mathrm{H}), 2.67-2.63(\mathrm{~m}, 1 \mathrm{H}), 2.59(\mathrm{dd}, J=18.5,2.7 \mathrm{~Hz}, 1$ H), 2.41 (dd, $J=18.4,6.5 \mathrm{~Hz}, 1 \mathrm{H}), 2.48-2.37$ (m, $1 \mathrm{H}), 2.06$ (s, $1 \mathrm{H}), 2.05-1.98$ (m, 1 H), $1.89(\mathrm{dd}, J=23,68,12.1 \mathrm{~Hz}, 2 \mathrm{H}), 1.83-1.75(\mathrm{~m}, 1 \mathrm{H}), 1.68(\mathrm{~d}, J=1.9 \mathrm{~Hz}, 3 \mathrm{H})$, 1.46 (s, $3 \mathrm{H}), 1.22 \mathrm{ppm}(\mathrm{s}, 3 \mathrm{H}) ;{ }^{13} \mathrm{C} \mathrm{NMR}\left(100 \mathrm{MHz}, \mathrm{CDCl}_{3}\right) \delta=208.0,172.1,138.4$, $115.7,89.9,86.9,68.4,67.5,65.6,65.5,63.3,61.4,53.2,49.8,37.0,36.8,35.3,27.2$, 22.5, 17.1, 8.2 ppm; HRMS (ESI): calcd for $\mathrm{C}_{21} \mathrm{H}_{27} \mathrm{O}_{5}{ }^{+}[\mathrm{M}+\mathrm{H}]^{+}$359.1853, found 359.1858.

\section{Preparation of cyclohexadienone 27':}
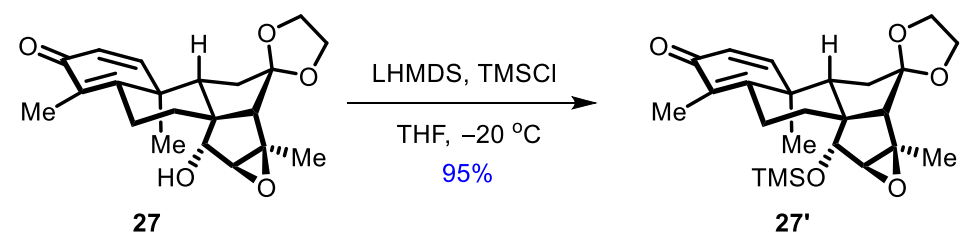

To a stirred solution of the alcohol 27 ( $0.50 \mathrm{~g}, 1.4 \mathrm{mmol}, 1.0$ equiv) in THF (20 $\mathrm{mL})$ at $-20{ }^{\circ} \mathrm{C}$ were sequentially added LiHMDS (1.0 M in THF, $2.1 \mathrm{~mL}, 2.1 \mathrm{mmol}$, 1.5 equiv) and TMSCl (0.27 mL, $2.1 \mathrm{mmol}, 1.5$ equiv). The resulting mixture was stirred at $-20{ }^{\circ} \mathrm{C}$ for 30 min before quenched with $\mathrm{NaHCO}_{3}(10 \mathrm{~mL}$, sat. aq.). The layers were separated, and the aqueous layer was extracted with EtOAc $(3 \times 10 \mathrm{~mL})$. The combined organic layers were dried $\left(\mathrm{Na}_{2} \mathrm{SO}_{4}\right)$ and concentrated in vacuo. Flash column chromatography (silica gel, hexanes:EtOAc 10:1) afforded cyclohexadienone 27' (0.57 
g, 95\%) as a white solid. 27': m.p. $159-161^{\circ} \mathrm{C}$ (hexanes/EtOAc); $R_{\mathrm{f}}=0.55$ (silica gel, hexanes:EtOAc 3:1); $[\alpha]_{\mathrm{D}}{ }^{20}=-19.4\left(\mathrm{c}=0.5, \mathrm{CHCl}_{3}\right) ;{ }^{1} \mathrm{H} \mathrm{NMR}\left(400 \mathrm{MHz}, \mathrm{CDCl}_{3}\right) \delta=$ $6.79(\mathrm{~d}, J=9.9 \mathrm{~Hz}, 1 \mathrm{H}), 6.17(\mathrm{~d}, J=9.9 \mathrm{~Hz}, 1 \mathrm{H}), 4.37(\mathrm{~s}, 1 \mathrm{H}), 3.97-3.87(\mathrm{~m}, 2 \mathrm{H})$, 3.86-3.79 (m, $1 \mathrm{H}), 3.73(\mathrm{q}, J=6.9 \mathrm{~Hz}, 1 \mathrm{H}), 3.39$ (s, $1 \mathrm{H}), 2.73-2.67(\mathrm{~m}, 1 \mathrm{H}), 2.60$ $2.50(\mathrm{~m}, 1 \mathrm{H}), 2.45-2.34(\mathrm{~m}, 1 \mathrm{H}), 2.11(\mathrm{~s}, 1 \mathrm{H}), 2.10-2.05(\mathrm{~m}, 1 \mathrm{H}), 1.88(\mathrm{~d}, J=0.9$ $\mathrm{Hz}, 3 \mathrm{H}), 1.73-1.64$ (m, $2 \mathrm{H}), 1.50$ (s, $3 \mathrm{H}), 1.19$ (s, $3 \mathrm{H}), 1.22-1.13$ (m, $1 \mathrm{H}), 0.19$ ppm $(\mathrm{s}, 9 \mathrm{H}) ;{ }^{13} \mathrm{C} \mathrm{NMR}\left(100 \mathrm{MHz}, \mathrm{CDCl}_{3}\right) \delta=186.0,161.6,155.2,128.8,126.5,116.5$, $77.4,66.2,64.7,64.2,63.5,63.4,54.7,52.5,43.4,39.9,39.4,25.2,22.7,16.8,10.7,1.1$ ppm (3C); HRMS (ESI): calcd for $\mathrm{C}_{24} \mathrm{H}_{34} \mathrm{NaO}_{5} \mathrm{Si}^{+}[\mathrm{M}+\mathrm{Na}]^{+} 453.2068$, found 453.2073.

\section{Photo-Nazarov cyclization of cyclohexadienone 27':}

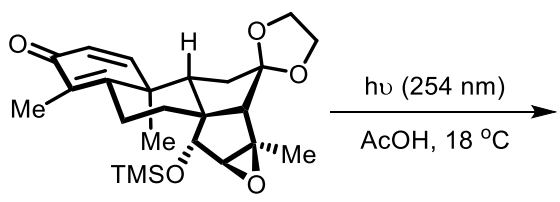

$27^{\prime}$

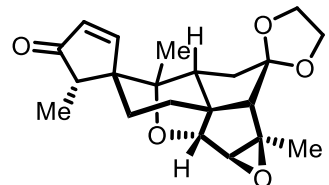

$30,10 \%$

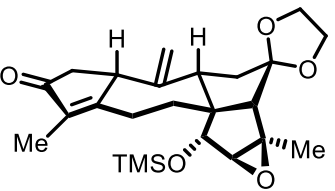

$34,5 \%$

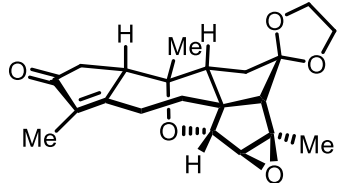

33, $56 \%$

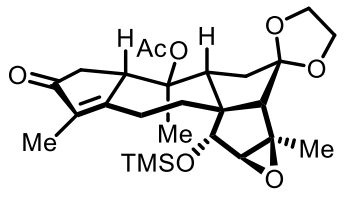

$35,12 \%$

A stirred solution of dienone $27^{\prime}(0.55 \mathrm{~g}, 1.3 \mathrm{mmol})$ in $\mathrm{AcOH}(64 \mathrm{~mL})$ at $18{ }^{\circ} \mathrm{C}$ was irradiated with a $254 \mathrm{~nm}$ lamp $(300 \mathrm{~W})$ for $40 \mathrm{~min}$ before concentrated in vacuo. The residue was partitioned between $\mathrm{CH}_{2} \mathrm{Cl}_{2}(20 \mathrm{~mL})$ and $\mathrm{NaHCO}_{3}(20 \mathrm{~mL}$, sat. aq.). The layers were separated, and the aqueous layer was extracted with $\mathrm{CH}_{2} \mathrm{Cl}_{2}(3 \times 20$ $\mathrm{mL})$. The combined organic layers were dried $\left(\mathrm{Na}_{2} \mathrm{SO}_{4}\right)$ and concentrated in vacuo. Flash column chromatography (silica gel, hexanes:EtOAc 8:1 to 4:1) afforded 
pentacycle $33(0.26 \mathrm{~g}, 56 \%)$ and spirocycle $\mathbf{3 0}$ (46 mg, 10\%), along with alkene $\mathbf{3 4}$ (28 mg, 5\%) and acetate 35 (75 mg, 12\%) as white solids. 34: m.p. $146-148{ }^{\circ} \mathrm{C}$ (hexanes/EtOAc); $R_{\mathrm{f}}=0.50$ (silica gel, hexanes:EtOAc 3:1); $[\alpha]_{\mathrm{D}}{ }^{20}=-25.3(\mathrm{c}=0.5$, $\left.\mathrm{CHCl}_{3}\right) ;{ }^{1} \mathrm{H} \mathrm{NMR}\left(400 \mathrm{MHz}, \mathrm{CDCl}_{3}\right) \delta=4.95(\mathrm{~s}, 1 \mathrm{H}), 4.85(\mathrm{~s}, 1 \mathrm{H}), 4.11(\mathrm{~s}, 1 \mathrm{H})$, 4.04-3.93 (m, 2 H), 3.92-3.82 (m, 2 H), 3.49 (d, J = 6.1 Hz, $1 \mathrm{H}), 3.22(\mathrm{~s}, 1 \mathrm{H}), 2.91$ (ddd, $J=18.3,12.2,4.8 \mathrm{~Hz}, 1 \mathrm{H}), 2.84-2.68(\mathrm{~m}, 2 \mathrm{H}), 2.68-2.58(\mathrm{~m}, 1 \mathrm{H}), 2.58-2.48$ (m, 2 H), $2.31(\mathrm{~s}, 1 \mathrm{H}), 2.22(\mathrm{~m}, 1 \mathrm{H}), 1.89(\mathrm{ddd}, J=14.6,12.5,4.8 \mathrm{~Hz}, 1 \mathrm{H}), 1.82-1.74$ $(\mathrm{m}, 1 \mathrm{H}), 1.64(\mathrm{~d}, J=1.7 \mathrm{~Hz}, 3 \mathrm{H}), 1.61(\mathrm{~s}, 3 \mathrm{H}), 0.03 \mathrm{ppm}(\mathrm{s}, 9 \mathrm{H}) ;{ }^{13} \mathrm{C}$ NMR $(100$ $\left.\mathrm{MHz}, \mathrm{CDCl}_{3}\right) \delta=208.4,174.3,144.6,134.8,115.0,110.1,74.6,67.8,66.3,65.1(2 \mathrm{C})$, 63.5, 57.3, 55.3, 49.1, 43.0, 40.0, 37.8, 27.3, 17.6, 8.2, 0.1 ppm (3C); HRMS (ESI): calcd for $\mathrm{C}_{24} \mathrm{H}_{35} \mathrm{O}_{5} \mathrm{Si}^{+}[\mathrm{M}+\mathrm{H}]^{+} 431.2248$, found 431.2250 .

35: m.p. $154-156^{\circ} \mathrm{C}$ (hexanes/EtOAc); $R_{\mathrm{f}}=0.50$ (silica gel, hexanes:EtOAc $\left.2: 1\right) ;[\alpha]_{\mathrm{D}}{ }^{20}$ $=+0.9\left(\mathrm{c}=0.5, \mathrm{CHCl}_{3}\right) ;{ }^{1} \mathrm{H} \mathrm{NMR}\left(400 \mathrm{MHz}, \mathrm{CDCl}_{3}\right) \delta=4.38-4.31(\mathrm{~m}, 1 \mathrm{H}), 4.25(\mathrm{~s}$, $1 \mathrm{H}), 4.01-3.95(\mathrm{~m}, 1 \mathrm{H}), 3.92-3.87(\mathrm{~m}, 1 \mathrm{H}), 3.87-3.81(\mathrm{~m}, 1 \mathrm{H}), 3.81-3.75(\mathrm{~m}, 1 \mathrm{H})$, $3.37(\mathrm{~s}, 1 \mathrm{H}), 3.33(\mathrm{dd}, J=14.9,6.6 \mathrm{~Hz}, 1 \mathrm{H}), 2.80-2.57(\mathrm{~m}, 2 \mathrm{H}), 2.53(\mathrm{dd}, J=14.88$, $12.04 \mathrm{~Hz}, 1 \mathrm{H}), 2.49-2.41(\mathrm{~m}, 1 \mathrm{H}), 2.35(\mathrm{~s}, 1 \mathrm{H}), 2.34(\mathrm{~s}, 1 \mathrm{H}), 2.33$ (s, $1 \mathrm{H}), 1.97$ (s, $3 \mathrm{H}), 1.79-1.70(\mathrm{~m}, 1 \mathrm{H}), 1.67(\mathrm{~d}, J=1.76,3 \mathrm{H}), 1.55(\mathrm{~s}, 3 \mathrm{H}), 1.53-1.46(\mathrm{~m}, 1 \mathrm{H})$, 0.95 (s, $3 \mathrm{H}), 0.15 \mathrm{ppm}(\mathrm{s}, 9 \mathrm{H}) ;{ }^{13} \mathrm{C} \mathrm{NMR}\left(100 \mathrm{MHz}, \mathrm{CDCl}_{3}\right) \delta=207.9,173.1,170.2$, 138.0, 115.1, 87.7, 74.5, 66.7, 64.6, 64.5, 64.0, 63.6, 56.9, 55.2, 49.7, 41.4, 37.3, 36.0, 26.7, 22.3, 20.0, 17.2, 8.3, 1.1 (3C) ppm; HRMS (ESI): calcd for $\mathrm{C}_{26} \mathrm{H}_{38} \mathrm{NaO}_{7} \mathrm{Si}^{+}[\mathrm{M}+$ $\mathrm{Na}]^{+}$513.2279, found 513.2284 


\section{Preparation of 2-methyl cyclopentenone 36:}

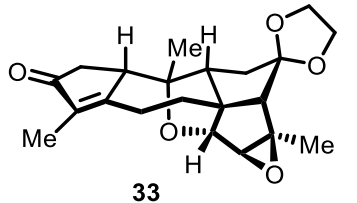

33

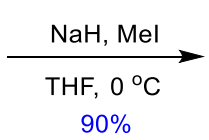

$90 \%$

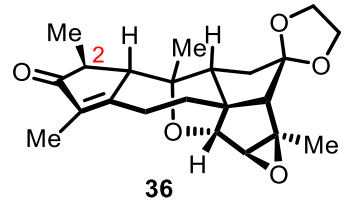

36

To a stirred solution of pentacycle 33 ( $11 \mathrm{mg}, 30 \mu \mathrm{mol}, 1.0$ equiv) in THF ( $2 \mathrm{~mL}$ ) at $0{ }^{\circ} \mathrm{C}$ were sequentially added $\mathrm{NaH}(60 \%$ dispersion in mineral oil, $2.4 \mathrm{mg}, 60 \mu \mathrm{mol}$, 2.0 equiv) and MeI (3.7 $\mu \mathrm{L}, 60 \mu \mathrm{mol}, 2.0$ equiv). The resulting mixture was stirred at 0 ${ }^{\circ} \mathrm{C}$ for 20 min before quenched with $\mathrm{NH}_{4} \mathrm{Cl}$ ( $2 \mathrm{~mL}$, sat. aq.). The layers were separated, and the aqueous layer was extracted with $\mathrm{Et}_{2} \mathrm{O}(3 \times 2 \mathrm{~mL})$. The combined organic layers were washed dried $\left(\mathrm{Na}_{2} \mathrm{SO}_{4}\right)$ and concentrated in vacuo. Flash column chromatography (silica gel, hexanes:EtOAc 8:1) afforded 2-methyl cyclopentenone $\mathbf{3 6}$ (10 mg, 90\%) as a white solid. 36: m.p. $198-200{ }^{\circ} \mathrm{C}$ (hexanes/EtOAc); $R_{\mathrm{f}}=0.50$ (silica gel, hexanes:EtOAc 3:1); $[\alpha]_{\mathrm{D}}{ }^{20}=-7.5\left(\mathrm{c}=0.1, \mathrm{CHCl}_{3}\right) ;{ }^{1} \mathrm{H} \mathrm{NMR}\left(400 \mathrm{MHz}, \mathrm{CDCl}_{3}\right) \delta=$ 4.02-3.91 (m, $4 \mathrm{H}), 3.67$ (s, $1 \mathrm{H}), 3.19$ (s, $1 \mathrm{H}), 2.86(\mathrm{dd}, J=12.9,6.8 \mathrm{~Hz}, 1 \mathrm{H}), 2.65$ $(\mathrm{dd}, J=11.7,7.3 \mathrm{~Hz}, 1 \mathrm{H}), 2.55-2.47(\mathrm{~m}, 1 \mathrm{H}), 2.44-2.33(\mathrm{~m}, 1 \mathrm{H}), 2.18(\mathrm{t}, J=2.1 \mathrm{~Hz}$, $1 \mathrm{H}), 2.06(\mathrm{t}, J=1.6 \mathrm{~Hz}, 1 \mathrm{H}), 2.04-1.98(\mathrm{~m}, 1 \mathrm{H}), 1.91(\mathrm{dd}, J=23.9,12.2 \mathrm{~Hz}, 1 \mathrm{H})$, 1.87-1.83 (m, $1 \mathrm{H}), 1.83-1.75(\mathrm{~m}, 1 \mathrm{H}), 1.69$ (d, $J=1.6 \mathrm{~Hz}, 3 \mathrm{H}), 1.46(\mathrm{~s}, 3 \mathrm{H}), 1.28$ $(\mathrm{s}, 3 \mathrm{H}), 1.19 \mathrm{ppm}(\mathrm{d}, J=7.6 \mathrm{~Hz}, 3 \mathrm{H}) ;{ }^{13} \mathrm{C} \mathrm{NMR}\left(100 \mathrm{MHz}, \mathrm{CDCl}_{3}\right) \delta=210.8,170.6$, 137.0, 115.7, 90.0, 87.3, 68.4, 67.5, 65.6 (2C), 63.3, 62.4, 61.4, 49.9, 41.8, 37.0, 35.2, 27.2, 22.3, 18.0, 17.2, 8.3 ppm; HRMS (ESI): calcd for $\mathrm{C}_{22} \mathrm{H}_{29} \mathrm{O}_{5}{ }^{+}[\mathrm{M}+\mathrm{H}]^{+}$373.2010, found 373.2019. 
Preparation of 4,4-dimethyl cyclopentanone 37:

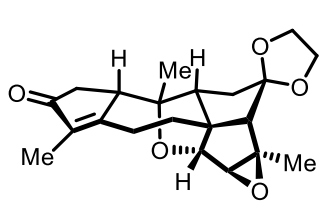

33

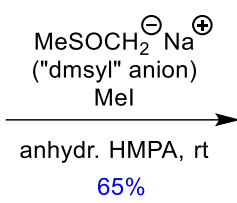

$65 \%$

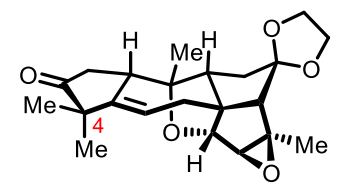

37

To a stirred solution of pentacycle $\mathbf{3 3}$ (20 mg, $56 \mu \mathrm{mol}, 1.0$ equiv) in freshly distilled HMPA ( $2 \mathrm{~mL})$ at room temperature were sequentially added "dmsyl" anion ${ }^{4}$ (0.5 M in DMSO, $0.16 \mathrm{~mL}, 84 \mu \mathrm{mol}, 1.5$ equiv) and $\mathrm{MeI}(6.8 \mu \mathrm{L}, 0.11 \mathrm{mmol}, 2.0$ equiv). The resulting mixture was stirred at room temperature for $5 \mathrm{~min}$ before quenched with $\mathrm{H}_{2} \mathrm{O}(2 \mathrm{~mL})$ and diluted with $\mathrm{Et}_{2} \mathrm{O}(2 \mathrm{~mL})$. The layers were separated, and the aqueous layer was extracted with $\mathrm{Et}_{2} \mathrm{O}(3 \times 2 \mathrm{~mL})$. The combined organic layers were washed with brine $(5 \mathrm{~mL})$, dried $\left(\mathrm{Na}_{2} \mathrm{SO}_{4}\right)$ and concentrated in vacuo. Flash column chromatography (silica gel, hexanes:EtOAc 8:1) afforded 4,4-dimethyl cyclopentanone $37(14 \mathrm{mg}, 65 \%)$ as a white solid. 37: m.p. $221-223{ }^{\circ} \mathrm{C}\left(\right.$ hexanes/EtOAc); $R_{\mathrm{f}}=0.40$ ( silica gel, hexanes:EtOAc 2:1); $[\alpha]_{\mathrm{D}}{ }^{20}=+55.1\left(\mathrm{c}=0.5, \mathrm{CHCl}_{3}\right) ;{ }^{1} \mathrm{H} \mathrm{NMR}(400 \mathrm{MHz}$, $\left.\mathrm{CDCl}_{3}\right) \delta=5.49-5.40(\mathrm{~m}, 1 \mathrm{H}), 4.07(\mathrm{~s}, 1 \mathrm{H}), 4.03-3.96(\mathrm{~m}, 2 \mathrm{H}), 3.96-3.91(\mathrm{~m}, 2 \mathrm{H})$, 3.26 (s, $1 \mathrm{H}), 2.82-2.71(\mathrm{~m}, 1 \mathrm{H}), 2.64-2.49$ (m, $3 \mathrm{H}), 2.39-2.25$ (m, $2 \mathrm{H}), 2.09-2.05$ (m, $1 \mathrm{H}), 1.97(\mathrm{t}, J=12.2 \mathrm{~Hz}, 1 \mathrm{H}), 1.86(\mathrm{ddd}, J=12.2,7.0,2.4 \mathrm{~Hz}, 1 \mathrm{H}), 1.51(\mathrm{~s}, 3 \mathrm{H})$, $1.21(\mathrm{~s}, 3 \mathrm{H}), 1.12(\mathrm{~s}, 3 \mathrm{H}), 1.06 \mathrm{ppm}(\mathrm{s}, 3 \mathrm{H}) ;{ }^{13} \mathrm{C} \mathrm{NMR}\left(100 \mathrm{MHz}, \mathrm{CDCl}_{3}\right) \delta=220.0$ $146.7,119.7,115.8,85.9,85.0,69.3,67.0,65.7,64.9,63.3,60.0,54.4,52.5,49.7,40.5$, 40.0, 37.2, 27.0, 23.6, 22.8, 17.4 ppm; HRMS (ESI): calcd for $\mathrm{C}_{22} \mathrm{H}_{29} \mathrm{O}_{5}{ }^{+}[\mathrm{M}+\mathrm{H}]^{+}$ 373.2010, found 373.2021. 


\section{Preparation of acetonide 38:}

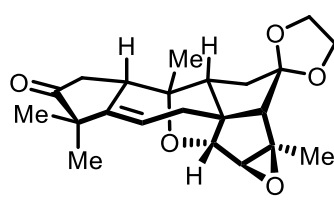

37

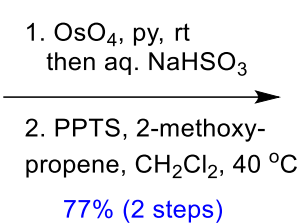

$77 \%$ (2 steps)

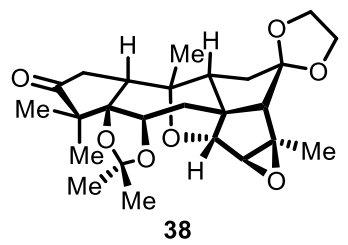

38

To a stirred solution of 4,4-dimethyl cyclopentanone $37(0.14 \mathrm{~g}, 0.38 \mathrm{mmol}, 1.0$ equiv) in pyridine $(5 \mathrm{~mL})$ at room temperature was added $\mathrm{OsO}_{4}(0.11 \mathrm{~g}, 0.41 \mathrm{mmol}, 1.1$ equiv). The resulting mixture was stirred at room temperature for $8 \mathrm{~h}$ before diluted with $\mathrm{Et}_{2} \mathrm{O}(5 \mathrm{~mL})$ and quenched with $\mathrm{NaHSO}_{3}(5 \mathrm{~mL}$, sat. aq.). The layers were separated, and the aqueous layer was extracted with $\mathrm{Et}_{2} \mathrm{O}(3 \times 5 \mathrm{~mL})$. The combined organic layers were dried $\left(\mathrm{Na}_{2} \mathrm{SO}_{4}\right)$ and concentrated in vacuo. Flash column chromatography (silica gel, hexanes:EtOAc 2:1) afforded the corresponding diol as a white solid.

To a stirred solution of diol (obtained above) in $\mathrm{CH}_{2} \mathrm{Cl}_{2}(5 \mathrm{~mL})$ at room temperature were sequentially added 2,2-methoxypropene $(0.11 \mathrm{~mL}, 1.1 \mathrm{mmol}, 3.0$ equiv) and PPTS (19 mg, $0.075 \mathrm{mmol}, 0.2$ equiv). The resulting mixture was warmed to $40{ }^{\circ} \mathrm{C}$ and stirred for $12 \mathrm{~h}$ before cooled to room temperature and quenched with $\mathrm{NaHCO}_{3}$ (5 mL, sat. aq.). The layers were separated, and the aqueous layer was extracted with $\mathrm{CH}_{2} \mathrm{Cl}_{2}(3 \times 5 \mathrm{~mL})$. The combined organic layers were dried $\left(\mathrm{Na}_{2} \mathrm{SO}_{4}\right)$ and concentrated in vacuo. Flash column chromatography (silica gel, hexanes:EtOAc 6:1) afforded acetonide 38 ( $0.13 \mathrm{~g}, 77 \%$ over two steps) as a white solid. 38: m.p. 233$235{ }^{\circ} \mathrm{C}$ (hexanes/EtOAc); $R_{\mathrm{f}}=0.45$ (silica gel, hexanes:EtOAc $\left.3: 1\right) ;[\alpha]_{\mathrm{D}}{ }^{20}=-10.9(\mathrm{c}$ $\left.=0.5, \mathrm{CHCl}_{3}\right) ;{ }^{1} \mathrm{H} \mathrm{NMR}\left(400 \mathrm{MHz}, \mathrm{CDCl}_{3}\right) \delta=4.18(\mathrm{dd}, J=6.1,1.3 \mathrm{~Hz}, 1 \mathrm{H}), 4.14(\mathrm{~s}$, $1 \mathrm{H}), 4.01-3.93(\mathrm{~m}, 4 \mathrm{H}), 3.41(\mathrm{dd}, J=12.2,7.3 \mathrm{~Hz}, 1 \mathrm{H}), 3.26(\mathrm{~s}, 1 \mathrm{H}), 2.83(\mathrm{dd}, J=$ 
17.9, 7.9 Hz, $1 \mathrm{H}), 2.64-2.55(\mathrm{~m}, 2 \mathrm{H}), 2.45(\mathrm{dd}, J=17.9,10.4 \mathrm{~Hz}, 1 \mathrm{H}), 2.11(\mathrm{~s}, 1 \mathrm{H})$, $2.09(\mathrm{dd}, J=15.9,6.1 \mathrm{~Hz}, 1 \mathrm{H}), 1.87(\mathrm{dd}, J=24.2,12.1 \mathrm{~Hz}, 1 \mathrm{H}), 1.86-1.78(\mathrm{~m}, 1 \mathrm{H})$, 1.50 (s, $3 \mathrm{H}), 1.44$ (s, $3 \mathrm{H}), 1.26$ (s, $3 \mathrm{H}), 1.18$ (s, $3 \mathrm{H}), 1.13$ (s, $3 \mathrm{H}), 1.08$ ppm (s, $3 \mathrm{H})$;

${ }^{13} \mathrm{C} \mathrm{NMR}\left(100 \mathrm{MHz}, \mathrm{CDCl}_{3}\right) \delta=217.9,115.8,105.7,92.5,89.5,87.7,76.1,69.7,67.7$, $65.5,64.0,63.6,61.9,57.0,52.2,49.1,40.2,36.6,35.8,28.3,26.2,24.7,22.8,18.5$, 17.5 ppm; HRMS (ESI): calcd for $\mathrm{C}_{25} \mathrm{H}_{34} \mathrm{NaO}_{7}^{+}[\mathrm{M}+\mathrm{Na}]^{+}$469.2197, found 469.2202.

\section{Preparation of enone 39:}
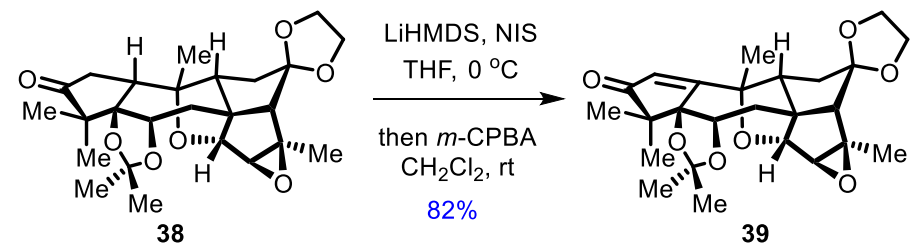

To a stirred solution of acetonide $\mathbf{3 8}(95 \mathrm{mg}, 0.21 \mathrm{mmol}, 1.0$ equiv) in THF ( $4 \mathrm{~mL}$ ) at $0{ }^{\circ} \mathrm{C}$ were sequentially added LiHMDS (1.0 M in THF, $0.25 \mathrm{~mL}, 0.25 \mathrm{mmol}, 1.2$ equiv) and NIS (72 mg, $0.32 \mathrm{mmol}, 1.5$ equiv). The resulting mixture was stirred at 0 ${ }^{\circ} \mathrm{C}$ for $1 \mathrm{~h}$ before $\mathrm{CH}_{2} \mathrm{Cl}_{2}(8 \mathrm{~mL}), \mathrm{NaHCO}_{3}(0.18 \mathrm{~g}, 2.1 \mathrm{mmol}, 10$ equiv) and $m$-CPBA ( $85 \%$ wt/wt, $0.21 \mathrm{~g}, 1.1 \mathrm{mmol}, 5.0$ equiv) were sequentially added. The resulting mixture was stirred at room temperature for $6 \mathrm{~h}$ before quenched with $\mathrm{Na}_{2} \mathrm{~S}_{2} \mathrm{O}_{3}(10 \mathrm{~mL}$, sat. aq.). The layers were separated, and the aqueous layer was extracted with $\mathrm{CH}_{2} \mathrm{Cl}_{2}$ $(3 \times 5 \mathrm{~mL})$. The combined organic layers were dried $\left(\mathrm{Na}_{2} \mathrm{SO}_{4}\right)$ and concentrated in vасио. Flash column chromatography (silica gel, hexanes:EtOAc 5:1) afforded enone 39 (103 mg, 82\%) as a white solid. 39: m.p. $216-218{ }^{\circ} \mathrm{C}$ (hexanes/EtOAc); $R_{\mathrm{f}}=0.45$ (silica gel, hexanes:EtOAc 3:1); $[\alpha] \mathrm{D}^{20}=-12.5\left(\mathrm{c}=0.1, \mathrm{CHCl}_{3}\right) ;{ }^{1} \mathrm{H} \mathrm{NMR}(400 \mathrm{MHz}$, $\left.\mathrm{CDCl}_{3}\right) \delta=6.40(\mathrm{~s}, 1 \mathrm{H}), 4.50(\mathrm{dd}, J=4.2,2.2 \mathrm{~Hz}, 1 \mathrm{H}), 4.09(\mathrm{dd}, J=11.5,7.1 \mathrm{~Hz}, 1$ H), 4.00-3.91 (m, $4 \mathrm{H}), 3.85$ (s, $1 \mathrm{H}), 3.30(\mathrm{~s}, 1 \mathrm{H}), 2.44(\mathrm{dd}, J=16.4,2.3 \mathrm{~Hz}, 1 \mathrm{H})$, 
$2.14(\mathrm{~s}, 1 \mathrm{H}), 1.96(\mathrm{dd}, J=16.3,4.1 \mathrm{~Hz}, 1 \mathrm{H}), 1.83(\mathrm{dd}, J=23.6,11.9 \mathrm{~Hz}, 1 \mathrm{H}), 1.82$ $1.75(\mathrm{~m}, 1 \mathrm{H}), 1.52(\mathrm{~s}, 3 \mathrm{H}), 1.51(\mathrm{~s}, 3 \mathrm{H}), 1.46(\mathrm{~s}, 3 \mathrm{H}), 1.35(\mathrm{~s}, 3 \mathrm{H}), 1.14 \mathrm{ppm}(\mathrm{s}, 6$ $\mathrm{H}) ;{ }^{13} \mathrm{C} \mathrm{NMR}\left(100 \mathrm{MHz}, \mathrm{CDCl}_{3}\right) \delta=210.1,179.2,132.1,116.1,107.8,92.5,90.8,85.4$, $77.4,69.1,66.1,65.6,64.2,63.6,63.0,52.2,44.9,39.3,35.7,27.7,26.5,24.7,23.3$, 19.4, 17.1 ppm; HRMS (ESI): calcd for $\mathrm{C}_{25} \mathrm{H}_{32} \mathrm{NaO}_{7}^{+}[\mathrm{M}+\mathrm{Na}]^{+} 467.2040$, found 467.2057.

Failed Attempts on the Inversion of the Stereochemistry at C-1 of Enone 39

Table S1. Attempted hydrogenation and 1,4-reduction of enone $\mathbf{3 9}^{a}$
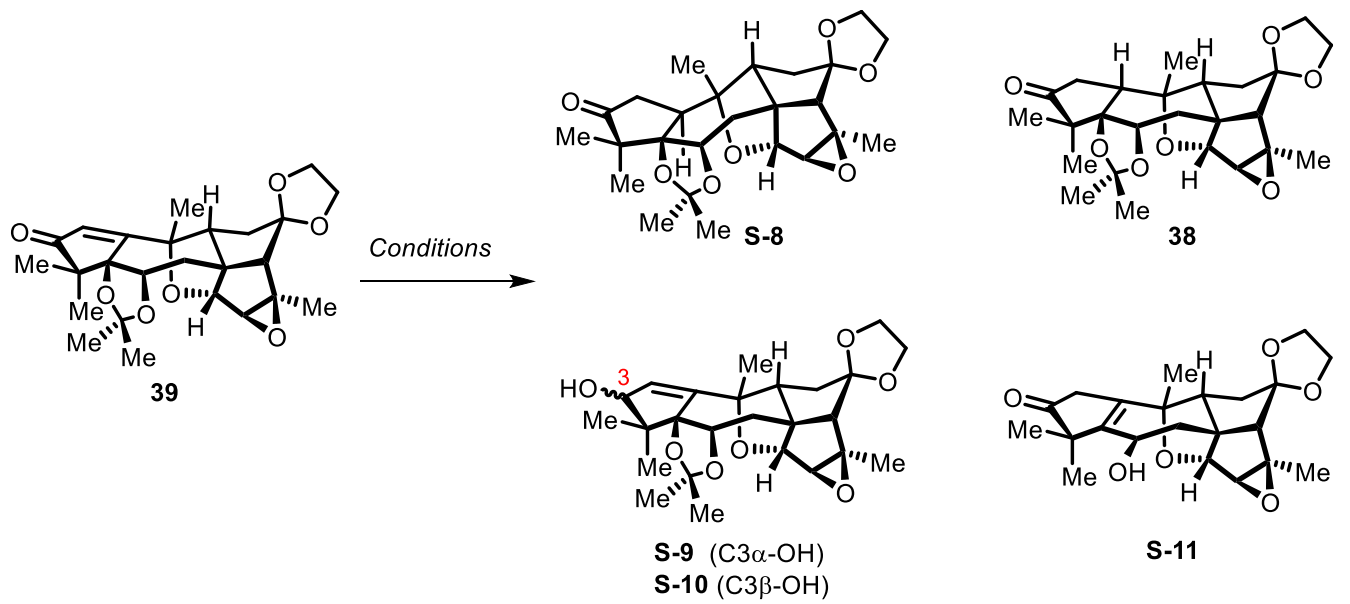

\begin{tabular}{|c|c|c|c|c|}
\hline Entry & Reagents & Solvent & Temp. $\left({ }^{\circ} \mathrm{C}\right)$ & Products (Yield) ${ }^{b}$ \\
\hline 1 & $\mathrm{Pd} / \mathrm{C}, \mathrm{H}_{2}(1 \mathrm{~atm})$ & $\mathrm{MeOH}$ & 25 & $-c$ \\
\hline 2 & $\mathrm{Pd} / \mathrm{C}, \mathrm{H}_{2}(50 \mathrm{~atm})$ & $\mathrm{MeOH}$ & 25 & $-^{c}$ \\
\hline 3 & Crabtree's cat., $\mathrm{H}_{2}(50 \mathrm{~atm})$ & $\mathrm{CH}_{2} \mathrm{Cl}_{2}$ & 25 & $-^{c}$ \\
\hline 4 & $\mathrm{PtO}_{2}, \mathrm{H}_{2}(50 \mathrm{~atm})$ & EtOAc & 25 & S-10 $(96 \%)$ \\
\hline 5 & $\mathrm{NaBH}_{4}$ & $\mathrm{MeOH}$ & 25 & S-9 (30\%), S-10 (45\%) \\
\hline 6 & $\mathrm{NaBH}_{4}, \mathrm{NiCl}_{2} \bullet 6 \mathrm{H}_{2} \mathrm{O}$ & $\mathrm{MeOH}$ & -20 & S-9 $(24 \%), \mathbf{S - 1 0}(72 \%)$ \\
\hline 7 & L-Selectride & THF & -40 & S-9 (62\%), S-10 (31\%) \\
\hline 8 & $\mathrm{LiAlH}_{4}, \mathrm{CuI}, \mathrm{HMPA}$ & THF & 0 & $-^{c}$ \\
\hline 9 & $\mathrm{HSiCl}_{3}, \mathrm{HMPA}$ & $\mathrm{CH}_{2} \mathrm{Cl}_{2}$ & 40 & $-^{c}$ \\
\hline 10 & {$\left[\left(\mathrm{Ph}_{3} \mathrm{P}\right) \mathrm{CuH}\right]_{6}$} & toluene & 60 & $--^{c}$ \\
\hline
\end{tabular}




\begin{tabular}{|c|c|c|c|c|}
\hline 11 & $\left(\mathrm{Ph}_{3} \mathrm{P}\right)_{3} \mathrm{RhCl}, \mathrm{Et}_{3} \mathrm{SiH}$ & toluene & 60 & $-^{c}$ \\
\hline 12 & $\mathrm{Mn}(\mathrm{dpm})_{3}, \mathrm{TBHP}, \mathrm{PhSiH}_{3}$ & $i$-PrOH & 25 & $38(80 \%)$ \\
\hline 13 & $\begin{array}{c}\mathrm{PhSiH}_{3}, n-\mathrm{Bu}_{3} \mathrm{SnH} \\
(\mathrm{O}-t-\mathrm{Bu})_{2}\end{array}$ & toluene & 120 & $-d$ \\
\hline 14 & $\mathrm{SmI}_{2}$ & $\mathrm{MeOH} / \mathrm{THF}$ & $\mathrm{rt}$ & S-11 $(10 \%)$ \\
\hline 15 & $\mathrm{Li}$, naphthalene & THF & -78 & S-11 $(53 \%)$ \\
\hline 16 & $\mathrm{Li}, \mathrm{NH}_{3}(\mathrm{l})$ & THF & -78 & $-d$ \\
\hline
\end{tabular}

$\overline{{ }^{a} \text { Reactions were performed on } 0.02 \mathrm{mmol} \text { scale. }{ }^{b} \text { Isolated yields. }{ }^{c} \text { Recovery of 39. }{ }^{d} \text { Decomposition. }}$

\section{Preparation of allylic alcohols S-9 and S-10 (entry 7):}

To a stirred solution of enone 39 ( $8.9 \mathrm{mg}, 20 \mu \mathrm{mol}, 1.0$ equiv) in THF (5 mL) at $40{ }^{\circ} \mathrm{C}$ was added L-Selectride (1.0 M in THF, $0.10 \mathrm{~mL}, 0.10 \mathrm{mmol}, 5.0$ equiv). The resulting mixture was stirred at $-40{ }^{\circ} \mathrm{C}$ for 30 min before quenched with $\mathrm{NH}_{4} \mathrm{Cl}(5 \mathrm{~mL}$, sat. aq.). The layers were separated, and the aqueous layer was extracted with EtOAc $(3 \times 5 \mathrm{~mL})$. The combined organic layers were dried $\left(\mathrm{Na}_{2} \mathrm{SO}_{4}\right)$ and concentrated in vacuo. Flash column chromatography (silica gel, hexanes:EtOAc 2:1) afforded allylic alcohols S-9 (5.5 mg, 62\%) and S-10 (2.8 mg, 31\%) as white solids. S-9: m.p. 222-224 ${ }^{\circ} \mathrm{C}\left(\right.$ hexanes/EtOAc); $R_{\mathrm{f}}=0.40$ (silica gel, hexanes:EtOAc $\left.1: 1\right) ;[\alpha]_{\mathrm{D}}{ }^{20}=-8.6(\mathrm{c}=0.1$, $\left.\mathrm{CHCl}_{3}\right) ;{ }^{1} \mathrm{H} \mathrm{NMR}\left(400 \mathrm{MHz}, \mathrm{CDCl}_{3}\right) \delta=6.18(\mathrm{~d}, J=1.5 \mathrm{~Hz}, 1 \mathrm{H}), 4.57(\mathrm{dd}, J=8.4$, $1.5 \mathrm{~Hz}, 1 \mathrm{H}), 4.19(\mathrm{dd}, J=4.2,2.0 \mathrm{~Hz}, 1 \mathrm{H}), 4.00$ (s, $1 \mathrm{H}), 3.99-3.91$ (m, $4 \mathrm{H}), 3.87$ (dd, $J=11.7,7.0 \mathrm{~Hz}, 1 \mathrm{H}), 3.32(\mathrm{~s}, 1 \mathrm{H}), 2.39(\mathrm{dd}, J=16.3,2.0 \mathrm{~Hz}, 1 \mathrm{H}), 2.12(\mathrm{~s}, 1 \mathrm{H}), 2.01$ $(\mathrm{dd}, J=16.1,4.2 \mathrm{~Hz}, 1 \mathrm{H}), 1.83(\mathrm{t}, J=11.9 \mathrm{~Hz}, 1 \mathrm{H}), 1.75(\mathrm{ddd}, J=12.2,7.0,2.0 \mathrm{~Hz}$, $1 \mathrm{H}), 1.51(\mathrm{~s}, 3 \mathrm{H}), 1.47$ (s, $3 \mathrm{H}), 1.42$ (s, $3 \mathrm{H}), 1.33$ (s, $3 \mathrm{H}), 1.15$ (s, $3 \mathrm{H}), 0.94$ (s, 3 $\mathrm{H}) ;{ }^{13} \mathrm{C} \mathrm{NMR}\left(100 \mathrm{MHz}, \mathrm{CDCl}_{3}\right) \delta=151.9,137.8,116.2,106.7,96.1,89.7,84.3,81.3$, 77.3, 68.9, 66.7, 65.5, 64.3, 63.6, 63.5, 51.3, 45.9, 39.4, 36.1, 28.6, 26.8, 24.4, 20.4, 19.3, 17.2 ppm; HRMS (ESI): calcd for $\mathrm{C}_{25} \mathrm{H}_{34} \mathrm{NaO}_{7}{ }^{+}[\mathrm{M}+\mathrm{Na}]^{+} 469.2197$, found 
469.2205 .

S-10: m.p. $229-230{ }^{\circ} \mathrm{C}$ (hexanes/EtOAc); $R_{\mathrm{f}}=0.45$ (silica gel, hexanes:EtOAc 1:1); $[\alpha]_{\mathrm{D}}^{20}=+8.5\left(\mathrm{c}=0.1, \mathrm{CHCl}_{3}\right) ;{ }^{1} \mathrm{H} \mathrm{NMR}\left(400 \mathrm{MHz}, \mathrm{CDCl}_{3}\right) \delta=6.45(\mathrm{~d}, J=3.2 \mathrm{~Hz}, 1$ H), $4.30(\mathrm{dd}, J=4.3,1.9 \mathrm{~Hz}, 1 \mathrm{H}), 4.00-3.93(\mathrm{~m}, 4 \mathrm{H}), 3.92-3.86(\mathrm{~m}, 1 \mathrm{H}), 3.89(\mathrm{~s}, 1$ H), $3.83(\mathrm{dd}, J=11.5,3.2 \mathrm{~Hz}, 1 \mathrm{H}), 3.30(\mathrm{~s}, 1 \mathrm{H}), 2.40(\mathrm{dd}, J=16.3,2.0 \mathrm{~Hz}, 1 \mathrm{H}), 2.12$ (s, $1 \mathrm{H}), 1.95(\mathrm{dd}, J=16.2,4.3 \mathrm{~Hz}, 1 \mathrm{H}), 1.82(\mathrm{t}, J=11.7 \mathrm{~Hz}, 1 \mathrm{H}), 1.76(\mathrm{ddd}, J=12.1$, 7.1, $2.2 \mathrm{~Hz}, 1 \mathrm{H}), 1.51$ (s, $3 \mathrm{H}), 1.47$ (s, $3 \mathrm{H}), 1.45$ (s, $3 \mathrm{H}), 1.34$ (s, $3 \mathrm{H}), 1.13(\mathrm{~s}, 3 \mathrm{H})$, $0.99 \mathrm{ppm}(\mathrm{s}, 3 \mathrm{H}) ;{ }^{13} \mathrm{C} \mathrm{NMR}\left(100 \mathrm{MHz}, \mathrm{CDCl}_{3}\right) \delta=155.4,136.8,116.2,107.0,95.9$, $89.8,84.3,81.7,76.3,68.9,66.7,65.5,64.2,63.6,63.4,48.2,45.6,39.6,36.0,28.5$, 27.0, 25.5, 24.2, 19.1, 17.2 ppm; HRMS (ESI): calcd for $\mathrm{C}_{25} \mathrm{H}_{34} \mathrm{NaO}_{7}^{+}[\mathrm{M}+\mathrm{Na}]^{+}$ 469.2197, found 469.2205.

\section{Conversion of enone 39 to acetonide 38 (entry 12):}

To a stirred solution of enone $39(8.9 \mathrm{mg}, 20 \mu \mathrm{mol}, 1.0$ equiv) in $i$-PrOH (4 mL, degassed) at room temperature were sequentially added $\mathrm{Mn}(\mathrm{dpm})_{3}(7.3 \mathrm{mg}, 10 \mu \mathrm{mol}$, 0.5 equiv) and TBHP (5.5 $\mathrm{M}$ in decane, $22 \mu \mathrm{L}, 0.12 \mathrm{mmol}, 6.0$ equiv). The resulting mixture was stirred at room temperature for $10 \mathrm{~h}$ before concentrated in vacuo. Flash column chromatography ( silica gel, hexanes:EtOAc 8:1) afforded acetonide $\mathbf{3 8}$ (7.1 mg, $80 \%)$

\section{Preparation of alcohol S-11 (entry 15):}

Preparation of the stock solution of $\mathrm{Li} /$ naphthalene (1.0 M in THF): To a stirred solution of naphthalene $(0.28 \mathrm{~g}, 2.2 \mathrm{mmol}, 1.1$ equiv) in THF (2 mL) at room 
temperature was added $\mathrm{Li}(14 \mathrm{mg}, 2.0 \mathrm{mmol}, 1.0$ equiv). The resulting mixture was stirred at room temperature for $1 \mathrm{~h}$ before use.

To a solution of enone $39\left(8.9 \mathrm{mg}, 20 \mu \mathrm{mol}, 1.0\right.$ equiv) in $\mathrm{THF}(5 \mathrm{~mL})$ at $-78{ }^{\circ} \mathrm{C}$ was added a solution of Li/naphthalene (1.0 M in THF, $0.20 \mathrm{~mL}, 0.20 \mathrm{mmol}, 10$ equiv). The resulting mixture was stirred at $-78{ }^{\circ} \mathrm{C}$ for 10 min before quenched with $\mathrm{NH}_{4} \mathrm{Cl}(5$ $\mathrm{mL}$, sat. aq.). The layers were separated, and the aqueous layer was extracted with EtOAc $(3 \times 5 \mathrm{~mL})$. The combined organic layers were dried $\left(\mathrm{Na}_{2} \mathrm{SO}_{4}\right)$ and concentrated in vacuo. Flash column chromatography (silica gel, hexanes:EtOAc 3:1) afforded alcohol S-11 (4.1 mg, 53\%) as a white solid. S-11: m.p. 217-219 ${ }^{\circ} \mathrm{C}$ (hexanes/EtOAc); $R_{\mathrm{f}}=0.30$ (silica gel, hexanes:EtOAc 1:1); $[\alpha]_{\mathrm{D}}{ }^{20}=-22.0\left(\mathrm{c}=0.1, \mathrm{CHCl}_{3}\right) ;{ }^{1} \mathrm{H} \mathrm{NMR}$ $\left(400 \mathrm{MHz}, \mathrm{CDCl}_{3}\right) \delta=4.38(\mathrm{~s}, 1 \mathrm{H}), 4.05-3.93(\mathrm{~m}, 4 \mathrm{H}), 3.80(\mathrm{~s}, 1 \mathrm{H}), 3.56(\mathrm{dd}, J=$ 11.6, 7.2 Hz, $1 \mathrm{H}), 3.35(\mathrm{~s}, 1 \mathrm{H}), 3.23(\mathrm{~d}, J=23.1 \mathrm{~Hz}, 1 \mathrm{H}), 3.01(\mathrm{~d}, J=23.0 \mathrm{~Hz}, 1 \mathrm{H})$, $2.27(\mathrm{~d}, J=15.3 \mathrm{~Hz}, 1 \mathrm{H}), 2.18-2.10(\mathrm{~m}, 2 \mathrm{H}), 1.97-1.82(\mathrm{~m}, 2 \mathrm{H}), 1.53(\mathrm{~s}, 3 \mathrm{H}), 1.34$ (s, $3 \mathrm{H}), 1.18$ (s, $3 \mathrm{H}), 1.08 \mathrm{ppm}(\mathrm{s}, 3 \mathrm{H}) ;{ }^{13} \mathrm{C} \mathrm{NMR}\left(100 \mathrm{MHz}, \mathrm{CDCl}_{3}\right) \delta=219.5,144.5$, $135.9,116.1,88.5,84.7,68.7,66.8,65.6,64.6,64.3,63.5,61.7,54.0,48.9,47.7,42.0$, 37.0, 23.7, 22.3, 20.3, 17.2 ppm; HRMS (ESI): calcd for $\mathrm{C}_{22} \mathrm{H}_{28} \mathrm{NaO}_{6}{ }^{+}[\mathrm{M}+\mathrm{Na}]^{+}$ 411.1778, found 411.1786.

\section{Preparation of alkene 41:}
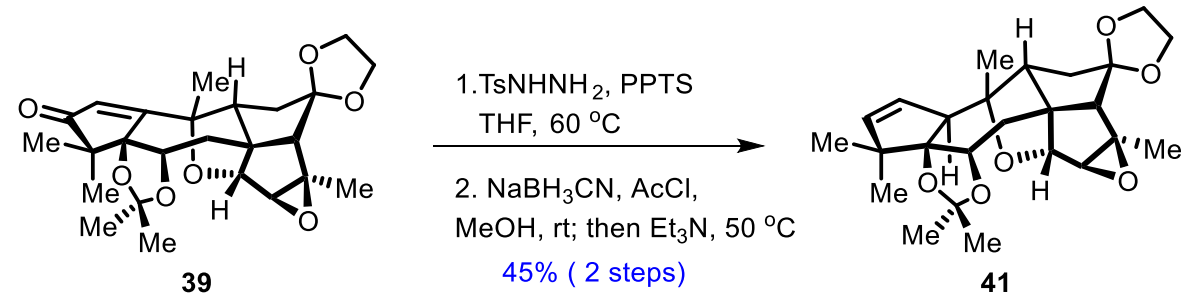

To a stirred solution of enone 39 (62 $\mathrm{mg}, 0.14 \mathrm{mmol}, 1.0$ equiv) in THF (5 mL) at 
room temperature were sequentially added $\mathrm{TsNHNH}_{2}(50 \mathrm{mg}, 0.27 \mathrm{mmol}, 2.0$ equiv) and PPTS (7.5 mg, $30 \mu$ mol, 0.2 equiv). The resulting mixture was warmed to $60{ }^{\circ} \mathrm{C}$ and stirred for $6 \mathrm{~h}$ before cooled to room temperature and quenched with $\mathrm{NaHCO}_{3}(5$ $\mathrm{mL}$, sat. aq.). The layers were separated, and the aqueous layer was extracted with EtOAc $(3 \times 5 \mathrm{~mL})$. The combined organic layers were dried $\left(\mathrm{Na}_{2} \mathrm{SO}_{4}\right)$ and concentrated in vacuo to afford the crude hydrazone as a white solid, which was used directly without further purification.

To a stirred solution of hydrazone (crude, obtained above) in $\mathrm{MeOH}(5 \mathrm{~mL})$ at room temperature were sequentially added $\mathrm{NaBH}_{3} \mathrm{CN}$ (26 mg, $0.41 \mathrm{mmol}, 1.5$ equiv) and $\mathrm{AcCl}(10 \mu \mathrm{L}, 0.14 \mathrm{mmol}, 1.0$ equiv). The resulting mixture was stirred at room temperature for $1 \mathrm{~h}$ before $\mathrm{Et}_{3} \mathrm{~N}(0.11 \mathrm{~mL}, 0.82 \mathrm{mmol}, 6.0$ equiv) was added. The resulting mixture was degassed and warmed to $50{ }^{\circ} \mathrm{C}$ and stirred for $1 \mathrm{~h}$ before cooled to room temperature and concentrated in vacuo. Flash column chromatography (silica gel, hexanes:EtOAc 10:1) afforded alkene 41 (27 mg, 45\% over two steps) as a white solid. 41: m.p. $243-245{ }^{\circ} \mathrm{C}$ (hexanes/EtOAc); $R_{\mathrm{f}}=0.40$ (silica gel, hexanes:EtOAc 4:1); $[\alpha]_{\mathrm{D}}^{20}=-3.3\left(\mathrm{c}=0.5, \mathrm{CHCl}_{3}\right) ;{ }^{1} \mathrm{H} \mathrm{NMR}\left(400 \mathrm{MHz}\right.$, acetone- $\left.d_{6}\right) \delta=5.88(\mathrm{dd}, J=6.2$, $1.8 \mathrm{~Hz}, 1 \mathrm{H}), 5.52(\mathrm{dd}, J=6.2,3.6 \mathrm{~Hz}, 1 \mathrm{H}), 4.38(\mathrm{t}, J=3.0 \mathrm{~Hz}, 1 \mathrm{H}), 4.07(\mathrm{~s}, 1 \mathrm{H})$, 3.96-3.91 (m, $4 \mathrm{H}), 3.85(\mathrm{dd}, J=11.1,7.8 \mathrm{~Hz}, 1 \mathrm{H}), 3.44-3.42(\mathrm{~m}, 1 \mathrm{H}), 3.19(\mathrm{~d}, J=$ $1.1 \mathrm{~Hz}, 1 \mathrm{H}), 2.36(\mathrm{dd}, J=16.1,2.7 \mathrm{~Hz}, 1 \mathrm{H}), 2.09$ (d, $J=1.6 \mathrm{~Hz}, 1 \mathrm{H}), 1.88(\mathrm{dd}, J=$ 16.1, 3.3 Hz, $1 \mathrm{H}), 1.72-1.67$ (m, $2 \mathrm{H}), 1.46$ (s, $3 \mathrm{H}), 1.46$ (d, J = 0.8 Hz, $3 \mathrm{H}), 1.30$ $1.29(\mathrm{~m}, 3 \mathrm{H}), 1.21(\mathrm{~s}, 3 \mathrm{H}), 1.13(\mathrm{~s}, 3 \mathrm{H}), 1.03 \mathrm{ppm}(\mathrm{s}, 3 \mathrm{H}) ;{ }^{13} \mathrm{C}$ NMR (100 MHz, acetone- $\left.d_{6}\right) \delta=141.0,127.7,117.0,108.5,99.6,94.1,87.2,78.6,70.2,66.0(2 \mathrm{C}), 65.5$, 
64.1, 63.8, 55.2, 51.2, 46.1, 43.8, 35.9, 29.2, 28.2, 24.2, 22.4, 20.0, 18.0 ppm; HRMS

(ESI): calcd for $\mathrm{C}_{25} \mathrm{H}_{34} \mathrm{NaO}_{6}{ }^{+}[\mathrm{M}+\mathrm{Na}]^{+}$453.2248, found 453.2257.

Of note, without the solvent degassing manipulations, the reaction on the same scale as described above afforded a mixture of alkene 41 (15 mg, 25\%) and alcohol S$10(14 \mathrm{mg}, 23 \%)$.

\section{Preparation of diene 42 and triene 43:}

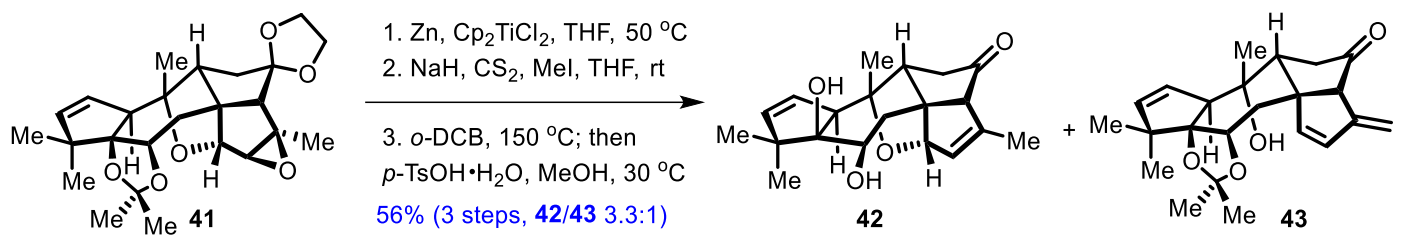

To a stirred solution of alkene 41 (22 $\mathrm{mg}, 50 \mu \mathrm{mol}, 1.0$ equiv) in THF ( $5 \mathrm{~mL}$, degassed) at room temperature were sequentially added $\mathrm{Cp}_{2} \mathrm{TiCl}_{2}(19 \mathrm{mg}, 75 \mu \mathrm{mol}, 1.5$ equiv) and $\mathrm{Zn}$ dust (12 mg, $0.19 \mathrm{mmol}, 3.8$ equiv). The resulting mixture was warmed to $50{ }^{\circ} \mathrm{C}$ and stirred for $6 \mathrm{~h}$ before filtered through a short pad of celite. The filtrate was washed with $\mathrm{NH}_{4} \mathrm{Cl}$ (5 mL, sat. aq.) and concentrated in vacuo. Flash column chromatography ( silica gel, hexanes:EtOAc 3:1) afforded the corresponding alcohol as a white solid.

To a stirred solution of the alcohol (obtained above), $\mathrm{CS}_{2}(12 \mu \mathrm{L}, 0.20 \mathrm{mmol}, 4.0$ equiv) in $\mathrm{THF}(5 \mathrm{~mL})$ at $0{ }^{\circ} \mathrm{C}$ was added $\mathrm{NaH}(60 \%$ dispersion in mineral oil, $8.0 \mathrm{mg}$, $0.20 \mathrm{mmol}, 4.0$ equiv). The resulting mixture was stirred at room temperature for $5 \mathrm{~h}$ before MeI (12 $\mu \mathrm{L}, 0.20 \mathrm{mmol}, 4.0$ equiv) was added. The reaction was stirred for an additional $10 \mathrm{~min}$ before quenched with $\mathrm{NH}_{4} \mathrm{Cl}$ (5 mL, sat. aq.). The layers were separated, and the aqueous layer was extracted with EtOAc $(3 \times 5 \mathrm{~mL})$. The combined organic layers were dried $\left(\mathrm{Na}_{2} \mathrm{SO}_{4}\right)$ and concentrated in vacuo to afford the crude 
xanthate as a white solid, which was used directly without further purification.

A stirred solution of xanthate (crude, obtained above) in $o$-DCB $(5 \mathrm{~mL})$ was warmed to $150{ }^{\circ} \mathrm{C}$ and stirred for $20 \mathrm{~h}$ before cooled to room temperature and $\mathrm{MeOH}$ (5 mL) and $p-\mathrm{TsOH} \cdot \mathrm{H}_{2} \mathrm{O}(19 \mathrm{mg}, 0.10 \mathrm{mmol}, 2.0$ equiv) were added. The resulting mixture was warmed to $30{ }^{\circ} \mathrm{C}$ and stirred for $36 \mathrm{~h}$ before diluted with EtOAc $(5 \mathrm{~mL})$ and quenched with $\mathrm{NaHCO}_{3}$ (5 mL, sat. aq.). The layers were separated, and the aqueous layer was extracted with EtOAc $(3 \times 5 \mathrm{~mL})$. The combined organic layers were dried $\left(\mathrm{Na}_{2} \mathrm{SO}_{4}\right)$ and concentrated in vacuo. Flash column chromatography (silica gel, hexanes:EtOAc 8:1) afforded diene $\mathbf{4 2}$ (7.1 mg, $43 \%$ over three steps) and triene $\mathbf{4 3}$ (2.4 mg, $13 \%$ over three steps) as white solids. 42: m.p. $230-232{ }^{\circ} \mathrm{C}$ (hexanes/EtOAc); $R_{\mathrm{f}}=0.40$ (silica gel, hexanes:EtOAc $\left.1: 1\right) ;[\alpha]_{\mathrm{D}}{ }^{20}=+145.0\left(\mathrm{c}=0.1, \mathrm{CHCl}_{3}\right) ;{ }^{1} \mathrm{H} \mathrm{NMR}$ $\left(400 \mathrm{MHz}, \mathrm{CDCl}_{3}\right) \delta=5.80(\mathrm{dd}, J=6.0,1.8 \mathrm{~Hz}, 1 \mathrm{H}), 5.56(\mathrm{q}, J=1.7 \mathrm{~Hz}, 1 \mathrm{H}), 5.53$ $(\mathrm{dd}, J=6.0,3.6 \mathrm{~Hz}, 1 \mathrm{H}), 4.82(\mathrm{~s}, 1 \mathrm{H}), 4.29(\mathrm{q}, J=8.3 \mathrm{~Hz}, 1 \mathrm{H}), 3.76(\mathrm{dd}, J=11.6$, $7.9 \mathrm{~Hz}, 1 \mathrm{H}), 3.21(\mathrm{dd}, J=3.6,1.8 \mathrm{~Hz}, 1 \mathrm{H}), 2.67(\mathrm{dd}, J=16.4,11.6 \mathrm{~Hz}, 1 \mathrm{H}), 2.60(\mathrm{~s}$, $1 \mathrm{H}), 2.17(\mathrm{~d}, J=1.5 \mathrm{~Hz}, 1 \mathrm{H}), 2.15-2.13(\mathrm{~m}, 1 \mathrm{H}), 2.13-2.06(\mathrm{~m}, 1 \mathrm{H}), 1.93(\mathrm{~d}, J=1.3$ $\mathrm{Hz}, 1 \mathrm{H}), 1.75(\mathrm{q}, J=1.3 \mathrm{~Hz}, 3 \mathrm{H}), 1.51(\mathrm{~d}, J=9.1 \mathrm{~Hz}, 1 \mathrm{H}), 1.32(\mathrm{~s}, 3 \mathrm{H}), 1.26(\mathrm{~s}, J=$ $2.2 \mathrm{~Hz}, 3 \mathrm{H}), 1.15 \mathrm{ppm}(\mathrm{s}, 3 \mathrm{H}) ;{ }^{13} \mathrm{C}$ NMR $\left(100 \mathrm{MHz}, \mathrm{CDCl}_{3}\right) \delta=214.8,144.3,141.0$, $127.8,127.7,88.8,87.9,87.8,72.7,69.7,58.9,58.7,54.0,46.1,41.9,39.2,23.1,22.6$, 21.2, 15.2 ppm; HRMS (ESI): calcd for $\mathrm{C}_{20} \mathrm{H}_{26} \mathrm{NaO}_{4}{ }^{+}[\mathrm{M}+\mathrm{Na}]^{+} 353.1723$, found 353.1728.

43: m.p. $174-176^{\circ} \mathrm{C}$ (hexanes/EtOAc); $R_{\mathrm{f}}=0.40$ ( silica gel, hexanes:EtOAc $\left.2: 1\right) ;[\alpha]_{\mathrm{D}}{ }^{20}$ $=+20.0\left(\mathrm{c}=0.1, \mathrm{CHCl}_{3}\right) ;{ }^{1} \mathrm{H} \mathrm{NMR}\left(600 \mathrm{MHz}\right.$, acetone- $\left.d_{6}\right) \delta=6.58(\mathrm{~d}, J=5.5 \mathrm{~Hz}, 1 \mathrm{H})$, 
$6.07(\mathrm{dd}, J=5.5,0.8 \mathrm{~Hz}, 1 \mathrm{H}), 5.96(\mathrm{dd}, J=6.2,1.7 \mathrm{~Hz}, 1 \mathrm{H}), 5.57(\mathrm{dd}, J=6.1,3.3 \mathrm{~Hz}$, $1 \mathrm{H}), 4.94(\mathrm{q}, J=1.1 \mathrm{~Hz}, 1 \mathrm{H}), 4.90(\mathrm{~d}, J=1.2 \mathrm{~Hz}, 1 \mathrm{H}), 4.45(\mathrm{dd}, J=4.5,1.8 \mathrm{~Hz}, 1$ H), 3.38-3.36 (m, $1 \mathrm{H}), 3.59(\mathrm{dd}, J=14.5,6.3 \mathrm{~Hz}, 1 \mathrm{H}), 3.37(\mathrm{~s}, 1 \mathrm{H}), 2.88(\mathrm{~d}, J=1.4$ Hz, $1 \mathrm{H}), 2.47$ (ddd, $J=16.5,14.6,0.8 \mathrm{~Hz}, 1 \mathrm{H}), 2.32(\mathrm{dd}, J=16.4,4.5 \mathrm{~Hz}, 1 \mathrm{H}), 2.17$ $(\mathrm{ddd}, J=16.4,6.3,1.6 \mathrm{~Hz}, 1 \mathrm{H}), 1.75(\mathrm{dd}, J=16.3,1.7 \mathrm{~Hz}, 1 \mathrm{H}), 1.46(\mathrm{~d}, J=0.8 \mathrm{~Hz}$, $3 \mathrm{H}), 1.35(\mathrm{~s}, 3 \mathrm{H}), 1.33(\mathrm{~d}, J=0.8 \mathrm{~Hz}, 3 \mathrm{H}), 1.15$ (s, $3 \mathrm{H}), 1.02 \mathrm{ppm}(\mathrm{s}, 3 \mathrm{H}) ;{ }^{13} \mathrm{C} \mathrm{NMR}$ $\left(150 \mathrm{MHz}\right.$, acetone- $\left.d_{6}\right) \delta=214.0,150.7,146.8,141.0,132.1,128.4,108.6,106.5,97.7$, 77.0, 73.9, 68.9, 59.8, 58.4, 51.3, 45.1, 38.3, 38.1, 28.1, 27.5, 25.0, 23.6, 23.0; HRMS (ESI): calcd for $\mathrm{C}_{23} \mathrm{H}_{30} \mathrm{NaO}_{4}{ }^{+}[\mathrm{M}+\mathrm{Na}]^{+}$393.2036, found 393.2048.

\section{Conversion of triene 43 to diene 42:}
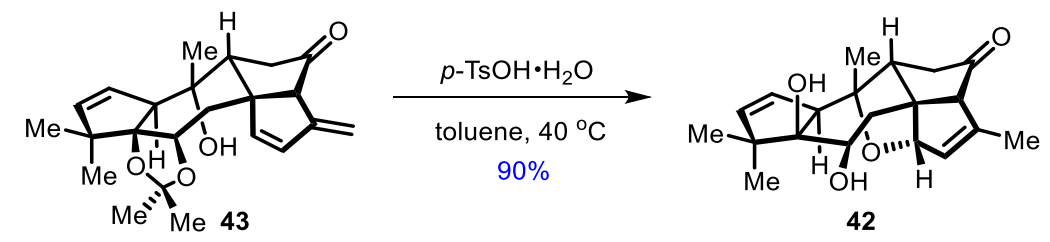

To a stirred solution of triene $\mathbf{4 3}(4.0 \mathrm{mg}, 11 \mu \mathrm{mol}, 1.0$ equiv) in toluene ( $2 \mathrm{~mL}$ ) at room temperature was added $p-\mathrm{TsOH} \cdot \mathrm{H}_{2} \mathrm{O}(2.1 \mathrm{mg}, 11 \mu \mathrm{mol}, 1.0$ equiv). The resulting mixture was warmed to $40{ }^{\circ} \mathrm{C}$ and stirred for $10 \mathrm{~h}$ before cooled to room temperature and quenched with $\mathrm{NaHCO}_{3}(2 \mathrm{~mL}$, sat. aq.). The layers were separated, and the aqueous layer was extracted with EtOAc $(3 \times 2 \mathrm{~mL})$. The combined organic layers were dried $\left(\mathrm{Na}_{2} \mathrm{SO}_{4}\right)$ and concentrated in vacuo. Flash column chromatography (silica gel, hexanes:EtOAc 8:1) afforded diene $\mathbf{4 2}$ (3.3 mg, 90\%). 


\section{Preparation of diene 46:}
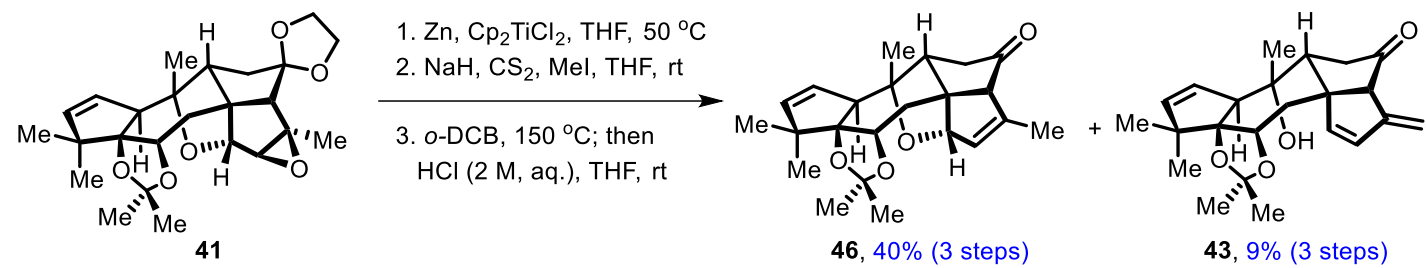

To a stirred solution of alkene 41 (5.2 $\mathrm{mg}, 12 \mu \mathrm{mol}, 1.0$ equiv) in THF ( $2 \mathrm{~mL}$, degassed) at room temperature were sequentially added $\mathrm{Cp}_{2} \mathrm{TiCl}_{2}(3.5 \mathrm{mg}, 14 \mu \mathrm{mol}, 1.2$ equiv) and $\mathrm{Zn}$ dust ( $3.8 \mathrm{mg}, 58 \mu \mathrm{mol}, 4.8$ equiv). The resulting mixture was warmed to $50{ }^{\circ} \mathrm{C}$ and stirred for $6 \mathrm{~h}$ before cooled to room temperature and filtered through a short pad of celite. The filtrate was washed with $\mathrm{NH}_{4} \mathrm{Cl}(5 \mathrm{~mL}$, sat. aq.) and concentrated in vacuo. Flash column chromatography (silica gel, hexanes:EtOAc 3:1) afforded the alcohol as a white solid.

To a stirred solution of alcohol (obtained above), $\mathrm{CS}_{2}(3.5 \mu \mathrm{L}, 58 \mu \mathrm{mol}, 4.8$ equiv) in $\mathrm{THF}(2 \mathrm{~mL})$ at $0{ }^{\circ} \mathrm{C}$ was added $\mathrm{NaH}(60 \%$ dispersion in mineral oil, $1.9 \mathrm{mg}, 47 \mu \mathrm{mol}$, 3.9 equiv). The resulting mixture was stirred at room temperature for $5 \mathrm{~h}$ before MeI (3.6 $\mu \mathrm{L}, 58 \mu \mathrm{mol}, 4.8$ equiv) was added. The reaction was stirred for an additional 10 min before quenched with $\mathrm{NH}_{4} \mathrm{Cl}(2 \mathrm{~mL}$, sat. aq. $)$. The layers were separated, and the aqueous layer was extracted with EtOAc $(3 \times 2 \mathrm{~mL})$. The combined organic layers were dried $\left(\mathrm{Na}_{2} \mathrm{SO}_{4}\right)$ and concentrated in vacuo to afford the crude xanthate as a white solid, which was used directly without further purification.

A stirred solution of xanthate (crude, obtained above) in $o$-DCB $(2 \mathrm{~mL})$ was warmed to $150{ }^{\circ} \mathrm{C}$ and stirred for $20 \mathrm{~h}$ before cooled to room temperature and THF (2 $\mathrm{mL})$ followed by $\mathrm{HCl}(2.0 \mathrm{M}$ aq., $0.20 \mathrm{~mL}, 0.40 \mathrm{mmol}, 33$ equiv) were added. The resulting mixture was stirred at room temperature for $24 \mathrm{~h}$ before diluted with EtOAc 
( $2 \mathrm{~mL})$ and quenched with $\mathrm{NaHCO}_{3}(2 \mathrm{~mL}$, sat. aq.). The layers were separated and the aqueous layer was extracted with EtOAc $(3 \times 2 \mathrm{~mL})$. The combined organic layers were dried $\left(\mathrm{Na}_{2} \mathrm{SO}_{4}\right)$ and concentrated in vacuo. Flash column chromatography (silica gel, hexanes:EtOAc 8:1) afforded diene $\mathbf{4 6}$ (1.8 $\mathrm{mg}, 40 \%$ over three steps) as a white solid, along with triene 43 (0.4 mg, 9\% over three steps). 46: m.p. $195-197{ }^{\circ} \mathrm{C}$ (hexanes/EtOAc); $R_{\mathrm{f}}=0.40$ ( silica gel, hexanes:EtOAc 2:1); $[\alpha]_{\mathrm{D}}{ }^{20}=-272.0(\mathrm{c}=0.1$, $\left.\mathrm{CHCl}_{3}\right) ;{ }^{1} \mathrm{H} \mathrm{NMR}\left(400 \mathrm{MHz}, \mathrm{CDCl}_{3}\right) \delta=5.99(\mathrm{dd}, J=6.2,1.7 \mathrm{~Hz}, 1 \mathrm{H}), 5.65(\mathrm{dd}, J=$ 6.1, $3.5 \mathrm{~Hz}, 1 \mathrm{H}), 5.51(\mathrm{~s}, 1 \mathrm{H}), 4.84(\mathrm{~s}, 1 \mathrm{H}), 4.48(\mathrm{t}, J=3.0 \mathrm{~Hz}, 1 \mathrm{H}), 4.05(\mathrm{dd}, J=$ 11.5, 7.9 Hz, $1 \mathrm{H}), 3.62(\mathrm{~s}, 1 \mathrm{H}), 2.75-2.68(\mathrm{~m}, 2 \mathrm{H}), 2.55(\mathrm{dd}, J=16.3,2.9 \mathrm{~Hz}, 1 \mathrm{H})$, $2.14(\mathrm{dd}, J=16.1,3.1 \mathrm{~Hz}, 1 \mathrm{H}), 2.07-2.00(\mathrm{~m}, 1 \mathrm{H}), 1.75-1.72(\mathrm{~m}, 3 \mathrm{H}), 1.50(\mathrm{~s}, 3 \mathrm{H})$, 1.37 (s, $3 \mathrm{H}), 1.32$ (s, $3 \mathrm{H}), 1.19$ (s, $3 \mathrm{H}), 1.06 \mathrm{ppm}(\mathrm{s}, 3 \mathrm{H}) ;{ }^{13} \mathrm{C}$ NMR (100 MHz, $\left.\mathrm{CDCl}_{3}\right) \delta=214.1,144.5,140.4,127.2,126.9,108.4,99.6,98.6,88.2,72.2,58.4,54.7$ 50.5 (2C), 42.5, 41.4, 36.3, 28.4, 27.9, 23.9, 22.1, 19.4, 15.1 ppm; HRMS (ESI): calcd for $\mathrm{C}_{23} \mathrm{H}_{30} \mathrm{NaO}_{4}{ }^{+}[\mathrm{M}+\mathrm{Na}]^{+}$393.2036, found 393.2030.

\section{Conversion of diene 46 to diene 42:}

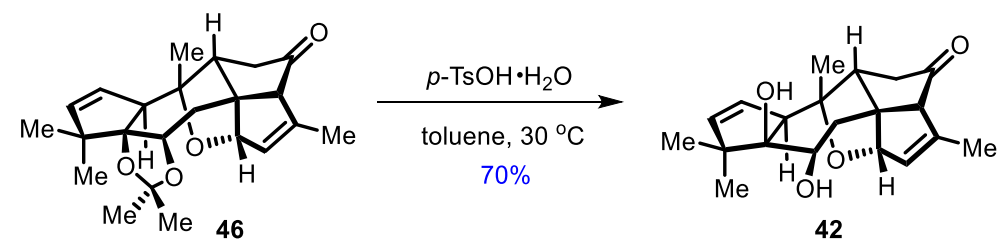

To a stirred solution of the diene $\mathbf{4 6}(3.7 \mathrm{mg}, 10 \mu \mathrm{mol}, 1.0$ equiv) in toluene ( $2 \mathrm{~mL}$ ) at room temperature was added $p-\mathrm{TsOH} \cdot \mathrm{H}_{2} \mathrm{O}(0.8 \mathrm{mg}, 4.0 \mu \mathrm{mol}, 0.4$ equiv). The resulting mixture was warmed to $30{ }^{\circ} \mathrm{C}$ and stirred for $10 \mathrm{~h}$ before cooled to room temperature and quenched with $\mathrm{NaHCO}_{3}(2 \mathrm{~mL}$, sat. aq.). The layers were separated, and the aqueous layer was extracted with EtOAc $(3 \times 2 \mathrm{~mL})$. The combined organic 
layers were dried $\left(\mathrm{Na}_{2} \mathrm{SO}_{4}\right)$ and concentrated in vacuo. Flash column chromatography (silica gel, hexanes:EtOAc 8:1) afforded diene $\mathbf{4 2}$ (2.3 mg, 70\%).

\section{Preparation of (-)-rhodomollanol A (7):}

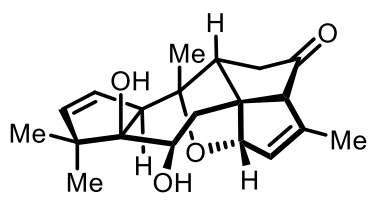

42

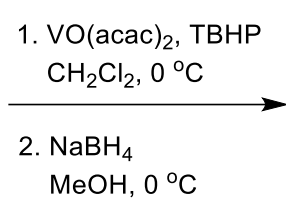

$74 \%$ (2 steps)

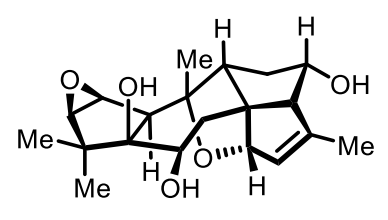

(-)-rhodomollanol A (7)

To a stirred solution of diene $\mathbf{4 2}$ (10 mg, $30 \mu \mathrm{mol}, 1.0$ eqiuv) in $\mathrm{CH}_{2} \mathrm{Cl}_{2}$ (4 mL) at $0{ }^{\circ} \mathrm{C}$ were sequentially added $4 \AA$ molecular sieve $(10 \mathrm{mg}), \mathrm{VO}(\mathrm{acac})_{2}(4.0 \mathrm{mg}, 15 \mu \mathrm{mol}$, 0.5 equiv) and TBHP (5.5 $\mathrm{M}$ in decane, $27 \mu \mathrm{L}, 0.15 \mathrm{mmol}, 5.0$ equiv). The resulting mixture was stirred at $0{ }^{\circ} \mathrm{C}$ for $2 \mathrm{~h}$ before quenched with $\mathrm{Na}_{2} \mathrm{~S}_{2} \mathrm{O}_{3}(4 \mathrm{~mL}$, sat. aq.). The layers were separated, and the aqueous layer was extracted with $\mathrm{CH}_{2} \mathrm{Cl}_{2}(3 \times 4 \mathrm{~mL})$. The combined organic layers were dried $\left(\mathrm{Na}_{2} \mathrm{SO}_{4}\right)$ and concentrated in vacuo. Flash column chromatography (silica gel, hexanes:EtOAc 3:1) afforded the corresponding epoxide as a white solid.

To a stirred solution of epoxide (obtained above) in $\mathrm{MeOH}(4 \mathrm{~mL})$ at $0{ }^{\circ} \mathrm{C}$ was added $\mathrm{NaBH}_{4}\left(4.6 \mathrm{mg}, 0.12 \mathrm{mmol}, 4.0\right.$ equiv). The resulting mixture was stirred at $0{ }^{\circ} \mathrm{C}$ for $15 \mathrm{~min}$ before diluted with $\mathrm{CH}_{2} \mathrm{Cl}_{2}(4 \mathrm{~mL})$, quenched with acetone $(1 \mathrm{~mL})$ and $\mathrm{H}_{2} \mathrm{O}$ (4 mL). The layers were separated, and the aqueous layer was extracted with $\mathrm{CH}_{2} \mathrm{Cl}_{2}$ (3 $\times 4 \mathrm{~mL})$. The combined organic layers were dried $\left(\mathrm{Na}_{2} \mathrm{SO}_{4}\right)$ and concentrated in vacuo. Reversed-phase HPLC purification [Ultimate XB-C18 (5 $\mu \mathrm{M}, 10$ × $250 \mathrm{~mm}$ column), $\left.\left.\mathrm{MeCN} / \mathrm{H}_{2} \mathrm{O}=30: 70, \mathrm{v}=1.5 \mathrm{~mL} \cdot \min ^{-1}, \lambda=210 \mathrm{~nm}, \mathrm{t}=12.5-15.0 \mathrm{~min}\right)\right]$ afforded $(-)-$ rhodomollanol A (7, $7.8 \mathrm{mg}, 74 \%$ over two steps) as a white solid. (-)-7: m.p. 185-186 ${ }^{\circ} \mathrm{C}\left(\mathrm{H}_{2} \mathrm{O} / \mathrm{MeOH}\right)$ [Lit. m.p. $\left.187-188^{\circ} \mathrm{C}(\mathrm{MeOH})\right],{ }^{5} R_{\mathrm{f}}=0.25$ (silica gel, hexanes:EtOAc 
$1: 2) ;[\alpha]_{\mathrm{D}}{ }^{20}=-64.0(\mathrm{c}=0.1, \mathrm{MeOH})\left[\right.$ Lit. $\left.[\alpha]_{\mathrm{D}}{ }^{20}=-44.9(\mathrm{c}=0.1, \mathrm{MeOH})\right] ;{ }^{5} \mathrm{H} \mathrm{NMR}$ $\left(400 \mathrm{MHz}, \mathrm{CD}_{3} \mathrm{OD}\right) \delta=5.49(\mathrm{~s}, 1 \mathrm{H}), 4.53(\mathrm{dd}, J=2.5,1.3 \mathrm{~Hz}, 1 \mathrm{H}), 4.20(\mathrm{ddd}, J=$ 11.7, 7.7, $5.4 \mathrm{~Hz}, 1 \mathrm{H}), 3.86(\mathrm{dd}, J=10.5,5.8 \mathrm{~Hz}, 1 \mathrm{H}), 3.83(\mathrm{dd}, J=3.3,1.0 \mathrm{~Hz}, 1 \mathrm{H})$, $3.46(\mathrm{dd}, J=11.8,6.4 \mathrm{~Hz}, 1 \mathrm{H}), 3.17(\mathrm{~d}, J=3.2 \mathrm{~Hz}, 1 \mathrm{H}), 2.46(\mathrm{~d}, J=7.7 \mathrm{~Hz}, 1 \mathrm{H})$, $2.29(\mathrm{~d}, J=1.0 \mathrm{~Hz}, 1 \mathrm{H}), 2.06(\mathrm{dd}, J=13.2,10.5 \mathrm{~Hz}, 1 \mathrm{H}), 1.88(\mathrm{~s}, 3 \mathrm{H}), 1.87(\mathrm{dd}, J=$ 13.2, $5.8 \mathrm{~Hz}, 1 \mathrm{H}), 1.73(\mathrm{ddd}, J=11.8,6.4,5.4 \mathrm{~Hz}, 1 \mathrm{H}), 1.62(\mathrm{q}, J=11.5 \mathrm{~Hz}, 1 \mathrm{H})$, 1.44 (s, $3 \mathrm{H}), 1.29$ (s, $3 \mathrm{H}), 1.10 \mathrm{ppm}(\mathrm{s}, 3 \mathrm{H}) ;{ }^{13} \mathrm{C} \mathrm{NMR}\left(100 \mathrm{MHz}, \mathrm{CD}_{3} \mathrm{OD}\right) \delta=148.3$, $127.9,88.4,88.2,82.6,74.8,73.9,64.6,64.4,61.7,59.5,55.9,48.8,48.4,43.1,35.4$, 22.4, 20.7, 20.6, 18.1 ppm; HRMS (ESI): calcd for $\mathrm{C}_{20} \mathrm{H}_{28} \mathrm{NaO}_{5}^{+}[\mathrm{M}+\mathrm{Na}]^{+}$371.1829, found 371.1834 [Lit. found 371.1825$].^{5}$ 
Table S2. ${ }^{1} \mathrm{H}$ NMR Spectroscopic $\left(\mathrm{CD}_{3} \mathrm{OD}, 25{ }^{\circ} \mathrm{C}\right)$ Comparison of Natural ${ }^{5}$ and Synthetic (-)-Rhodomollanol A (7).

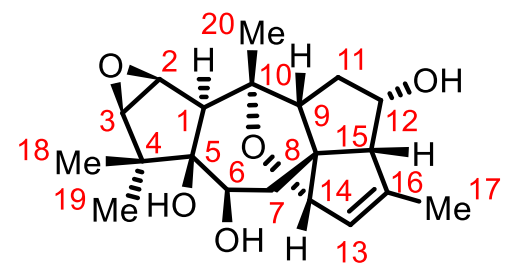

(-)-rhodomollanol A (7)

\begin{tabular}{ccc}
\hline No. & Natural & Synthetic \\
& $\delta{ }^{1} \mathrm{H}[\mathrm{ppm}$, mult, $J(\mathrm{~Hz})]$ & $\delta{ }^{1} \mathrm{H}[\mathrm{ppm}$, mult,$J(\mathrm{~Hz})]$ \\
\hline 1 & $2.29, \mathrm{~d}(J=0.6 \mathrm{~Hz})$ & $2.29, \mathrm{~d}(J=1.0 \mathrm{~Hz})$ \\
2 & $3.82, \mathrm{dd}(J=3.2,0.6 \mathrm{~Hz})$ & $3.83, \mathrm{dd}(J=3.3,1.0 \mathrm{~Hz})$ \\
3 & $3.17, \mathrm{~d}(J=3.2 \mathrm{~Hz})$ & $3.17, \mathrm{~d}(J=3.2 \mathrm{~Hz})$ \\
4 & & \\
5 & & $3.86, \mathrm{dd}(J=10.5,5.8 \mathrm{~Hz})$ \\
6 & $3.86, \mathrm{dd}(J=10.6,5.8 \mathrm{~Hz})$ & $1.87, \mathrm{dd}(J=13.2,5.8 \mathrm{~Hz})$ \\
7 & $1.87, \mathrm{dd}(J=13.2,5.8 \mathrm{~Hz})$ & $2.06, \mathrm{dd}(J=13.2,10.5 \mathrm{~Hz})$ \\
8 & $2.05, \mathrm{dd}(J=13.2,10.6 \mathrm{~Hz})$ & $3.46, \mathrm{dd}(J=11.8,6.4 \mathrm{~Hz})$ \\
9 & $3.46, \mathrm{dd}(J=12.0,6.4 \mathrm{~Hz})$ & $1.73, \mathrm{ddd}(J=11.8,6.4,5.4 \mathrm{~Hz})$ \\
10 & & $1.62, \mathrm{q}(J=11.5 \mathrm{~Hz})$ \\
11 & $1.72, \mathrm{ddd}(J=12.0,6.4,5.6 \mathrm{~Hz})$ & $5.49, \mathrm{~s}$ \\
& $1.63, \mathrm{q}(J=12.0 \mathrm{~Hz})$ & $\mathrm{ddd}(J=11.7,7.7,5.4 \mathrm{~Hz})$ \\
12 & $4.20, \mathrm{ddd}(J=12.0,7.8,5.6 \mathrm{~Hz})$ & $5.53, \mathrm{dd}(J=2.5,1.3 \mathrm{~Hz})$ \\
13 & $5.49, \mathrm{~s}$ & $2.46, \mathrm{~d}(J=7.7 \mathrm{~Hz})$ \\
14 & $4.53, \mathrm{~s}$ & $1.88, \mathrm{~s}$ \\
15 & $2.46, \mathrm{~d}(J=7.8 \mathrm{~Hz})$ & $1.29, \mathrm{~s}$ \\
16 & & $1.10, \mathrm{~s}$ \\
17 & $1.88, \mathrm{~s}$ & $1.44, \mathrm{~s}$ \\
18 & $1.29, \mathrm{~s}$ & \\
19 & $1.10, \mathrm{~s}$ & \\
20 & $1.44, \mathrm{~s}$ & \\
\hline
\end{tabular}


Table S3. ${ }^{13} \mathrm{C}$ NMR $\left(\mathrm{CD}_{3} \mathrm{OD}, 25{ }^{\circ} \mathrm{C}\right)$ Spectroscopic Comparison of Natural ${ }^{5}$ and Synthetic (-)-Rhodomollanol A (7).

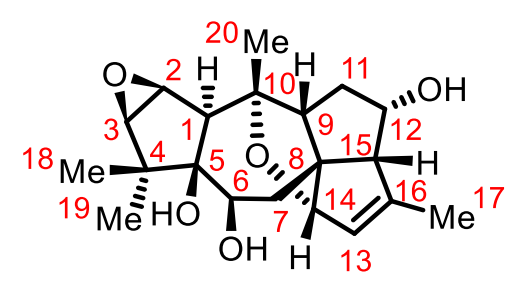

(-)-rhodomollanol A (7)

\begin{tabular}{ccc}
\hline No. & Natural & Synthetic \\
& ${ }^{13} \mathrm{C}(\mathrm{ppm})$ & ${ }^{13} \mathrm{C}(\mathrm{ppm})$ \\
\hline 1 & 55.9 & 55.9 \\
2 & 59.5 & 59.5 \\
3 & 64.7 & 64.6 \\
4 & 48.4 & 48.4 \\
5 & 82.6 & 82.6 \\
6 & 73.9 & 73.9 \\
7 & 43.1 & 43.1 \\
8 & 61.7 & 61.7 \\
9 & 49.0 & 48.8 \\
10 & 88.2 & 88.2 \\
11 & 35.4 & 35.4 \\
12 & 74.8 & 74.8 \\
13 & 128.0 & 128.0 \\
14 & 88.4 & 88.4 \\
15 & 64.4 & 64.4 \\
16 & 148.3 & 148.3 \\
17 & 18.1 & 18.1 \\
18 & 20.7 & 20.7 \\
19 & 20.6 & 20.6 \\
20 & 22.4 & 22.4 \\
\hline
\end{tabular}




\section{III) Abbreviations}

\begin{tabular}{|c|c|}
\hline acac & acetylacetonate \\
\hline AIBN & azodiisobutyronitrile \\
\hline $\mathrm{Bz}$ & benzoyl \\
\hline $\mathrm{Cp}$ & cyclopentadienyl \\
\hline DHP & 3,4-dihydro- $2 H$-pyran \\
\hline DIAD & diisopropyl azodicarboxylate \\
\hline DIBAL-H & diisobutyl aluminium hydride \\
\hline DMSO & dimethyl sulfoxide \\
\hline dpm & $\operatorname{tris}(2,2,6,6$-tetramethyl-3,5-heptanedionato) \\
\hline HFIP & 1,1,1,3,3,3-hexafluoro-2-propanol \\
\hline HMPA & hexamethylphosphoric triamide \\
\hline IBX & 2-iodoxybenzoic acid \\
\hline LiHMDS & lithium bis(trimethylsilyl)amide \\
\hline$m$-CPBA & 3-chloroperbenzoic acid \\
\hline $\mathrm{MOMCl}$ & chloromethyl methyl ether \\
\hline NaHMDS & sodium bis(trimethylsilyl)amide \\
\hline NIS & $N$-iodosuccinimide \\
\hline$o-\mathrm{DCB}$ & 1,2-dichlorobenzene \\
\hline oxone & potassium peroxymonosulfate \\
\hline PDC & pyridinium dichromate \\
\hline PIFA & [bis(trifluoroacetoxy)iodo]benzene \\
\hline
\end{tabular}




$\begin{array}{ll}\text { PPTS } & \text { pyridinium 4-toluenesulfonate } \\ p \text {-TsOH } & \text { 4-toluenesulfonic acid } \\ \text { py } & \text { pyridine } \\ \text { TBHP } & \text { tert-butyl hydroperoxide } \\ \text { TESCl } & \text { triethyl chlorosilane } \\ \text { TFA } & \text { trifluoroacetic acid } \\ \text { THF } & \text { tetrahydrofuran } \\ \text { TMSCl } & \text { trimethyl chlorosilane } \\ \text { TMSOTf } & \text { trimethylsilyl trifluoromethanesulfonate }\end{array}$

\section{IV) References}

(1) He, C.; Hu, J.; Wu. Y.; Ding, H. J. Am. Chem. Soc. 2017, 139, 6098-6101.

(2) $\mathbf{1 6}$ is commercially available, and could also be prepared according to the procedures reported by Shenvi and coworkers: Wan, K. K.; Iwasaki, K.; Umotoy, J. C.; Wolan, D. W.; Shenvi, R. A. Angew. Chem., Int. Ed. 2015, 54, 2410-2415.

(3) For the preparation of the known bromophenol S-4, see: Börger, C.; Schmidt, A. H.; Knölker, H. -J. Synlett 2014, 25, 1381-1384.

(4) For the preparation of "dmsyl” anion, see: Stork, G.; Niu, D.; Fujimoto, A.; Koft, E.

R.; Balkovec, J. M.; Tata, J. R.; Dake, G. R. J. Am. Chem. Soc. 2001, 123, 3239-3242.

(5) Zhou, J.; Zhan G.; Zhang, H.; Zhang, Q.; Li, Y.; Xue, Y.; Yao, G. Org. Lett. 2017, 19, 3935-3938. 


\section{IV) ${ }^{1} \mathrm{H}$ and ${ }^{13} \mathrm{C}$ NMR Spectra of Compounds}

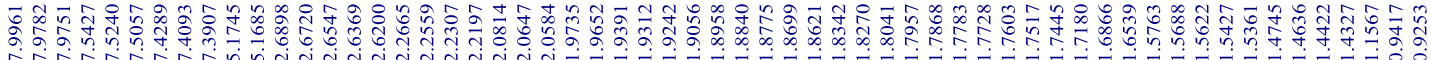

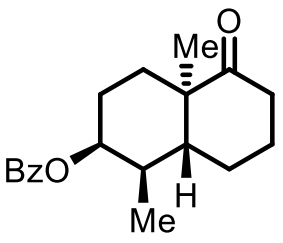

S-2

${ }^{1} \mathrm{H}$ NMR spectrum $\left(400 \mathrm{MHz}, \mathrm{CDCl}_{3}\right)$

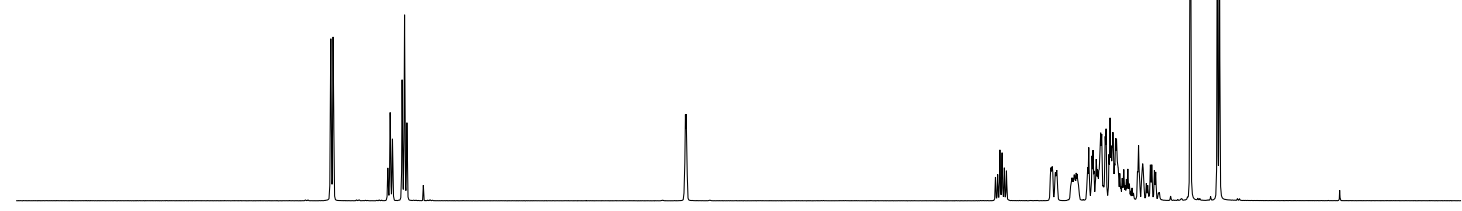

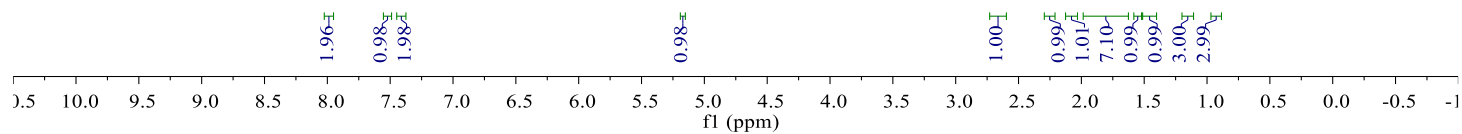

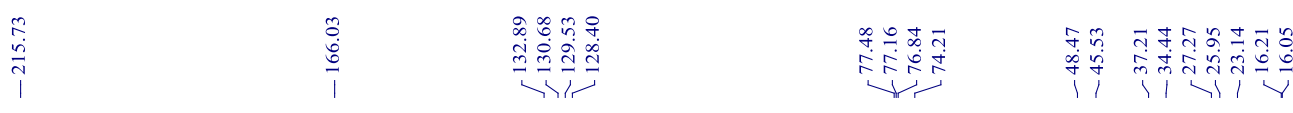

${ }^{13} \mathrm{C}$ NMR spectrum $\left(100 \mathrm{MHz}, \mathrm{CDCl}_{3}\right)$

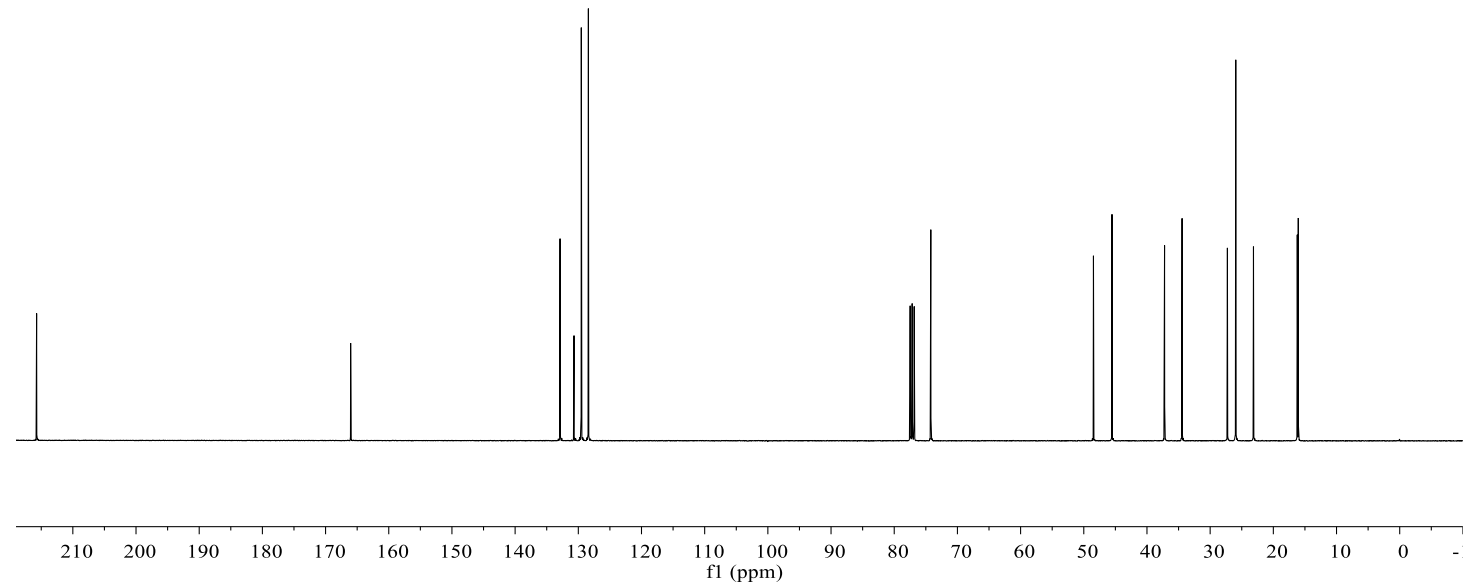




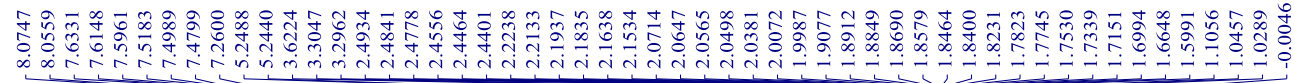

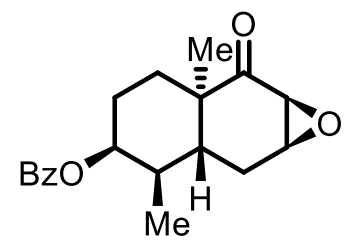

S-3

${ }^{1} \mathrm{H}$ NMR spectrum $\left(400 \mathrm{MHz}, \mathrm{CDCl}_{3}\right)$

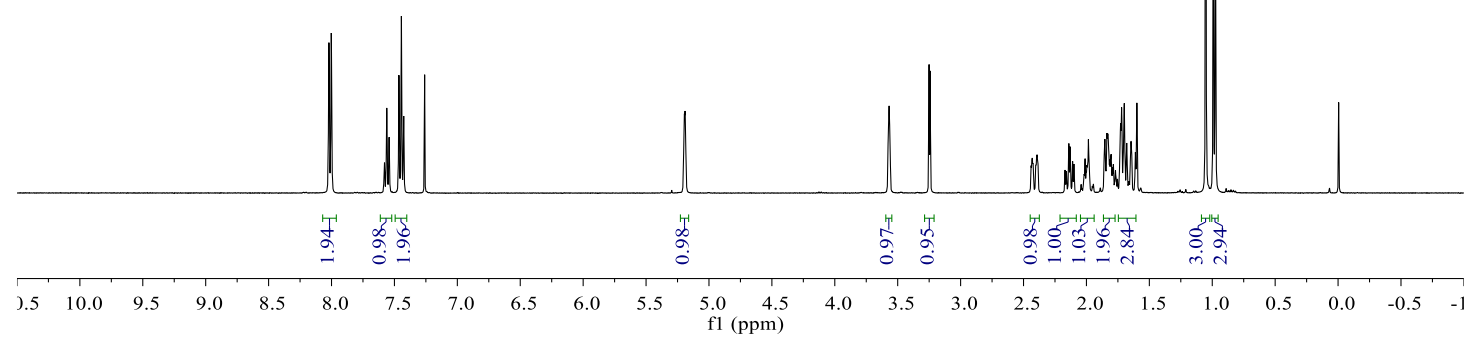

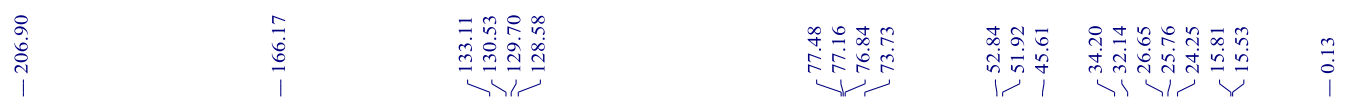

${ }^{13} \mathrm{C}$ NMR spectrum $\left(100 \mathrm{MHz}, \mathrm{CDCl}_{3}\right)$

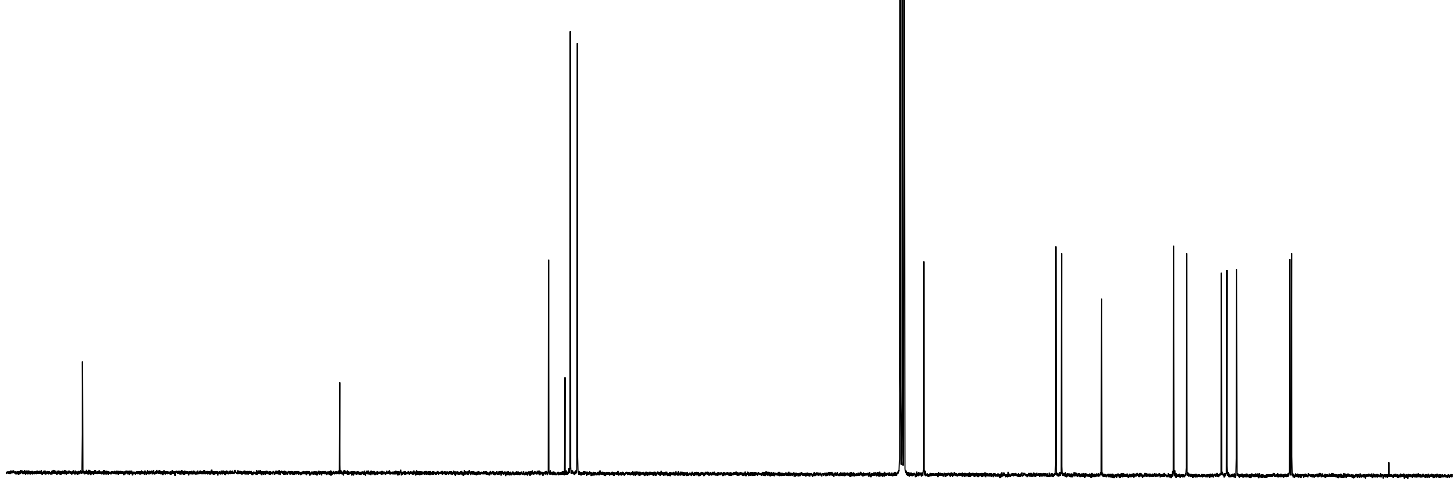

$\begin{array}{lllllllllllllllllllllll}210 & 200 & 190 & 180 & 170 & 160 & 150 & 140 & 130 & 120 & 110 & 100 & 90 & 80 & 70 & 60 & 50 & 40 & 30 & 20 & 10 & 0 & \end{array}$ 


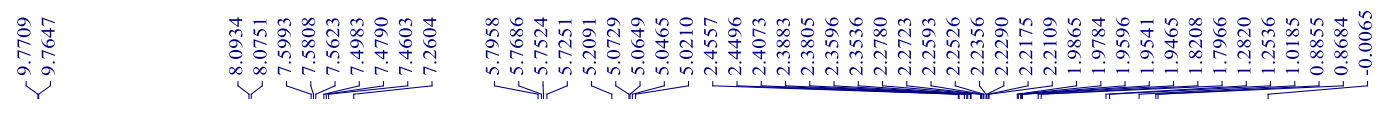<smiles>C=C[C@]1(C)CC[C@@H](OC(=O)c2ccccc2)C(C(C)C)[C@]1(C)CC=O</smiles>

15

${ }^{1} \mathrm{H}$ NMR spectrum $\left(400 \mathrm{MHz}, \mathrm{CDCl}_{3}\right)$

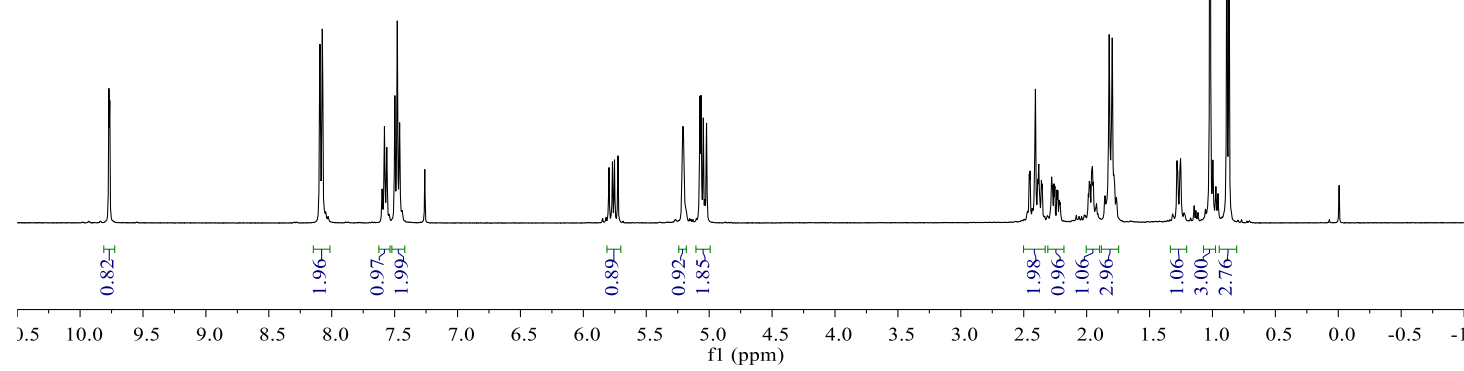

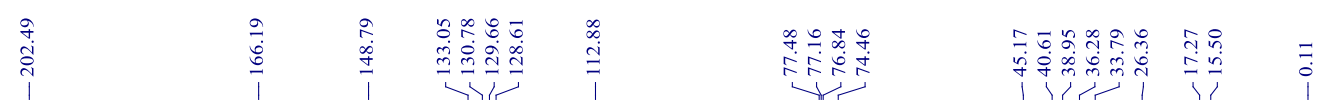

${ }^{13} \mathrm{C}$ NMR spectrum $\left(100 \mathrm{MHz}, \mathrm{CDCl}_{3}\right)$

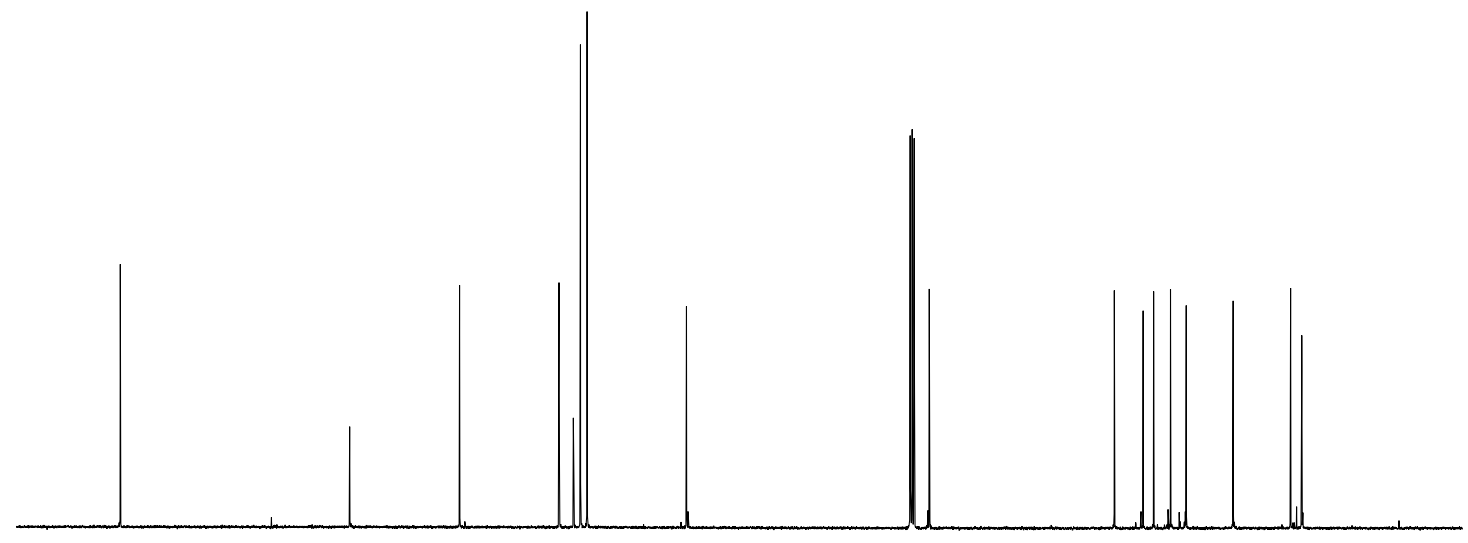

$\begin{array}{lllllllllllllllllllllll}210 & 200 & 190 & 180 & 170 & 160 & 150 & 140 & 130 & 120 & \begin{array}{c}110 \\ \mathrm{f} 1(\mathrm{ppm})\end{array} & 90 & 80 & 70 & 60 & 50 & 40 & 30 & 20 & 10 & 0 & -\end{array}$ 


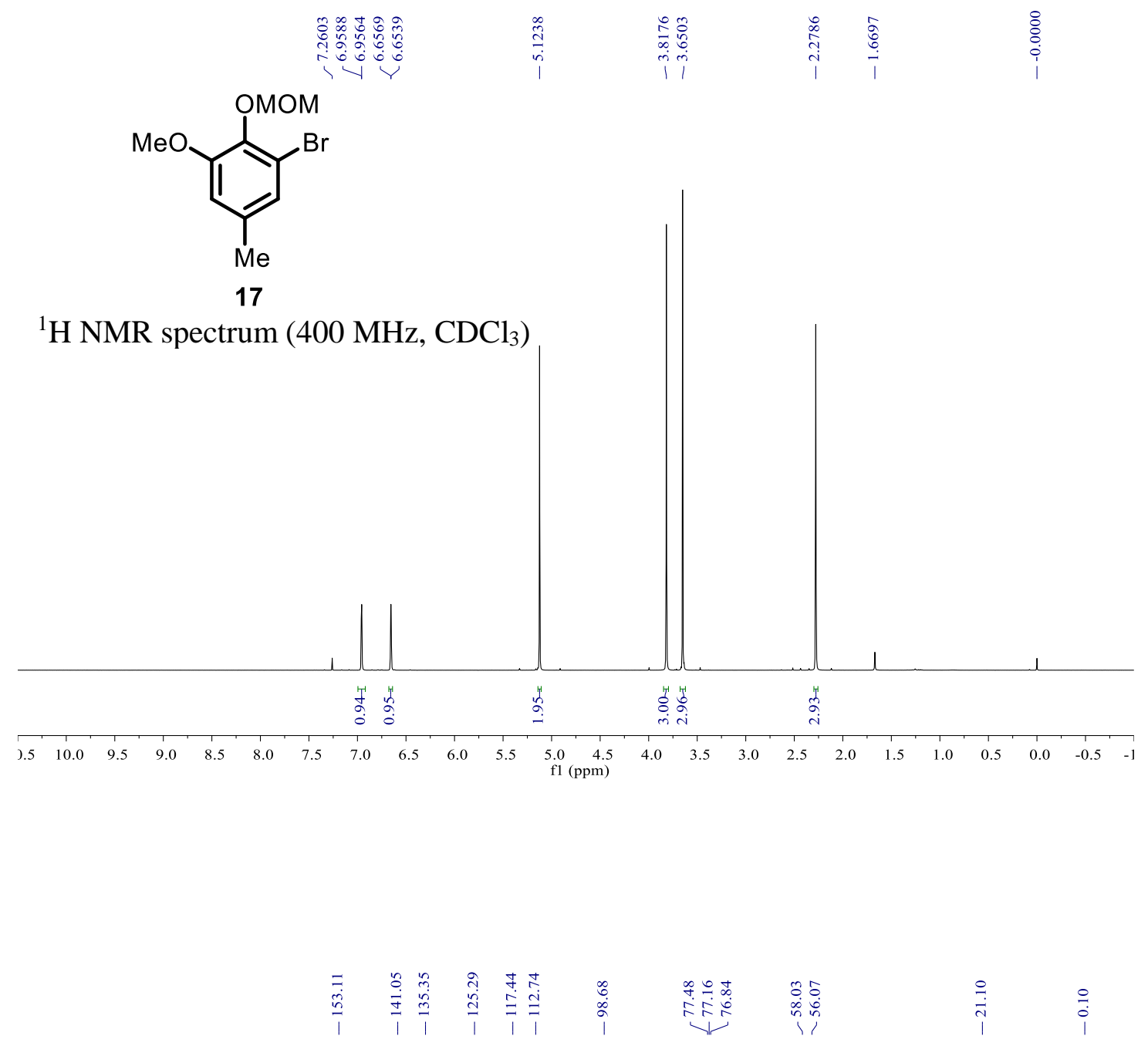

${ }^{13} \mathrm{C}$ NMR spectrum $\left(100 \mathrm{MHz}, \mathrm{CDCl}_{3}\right)$

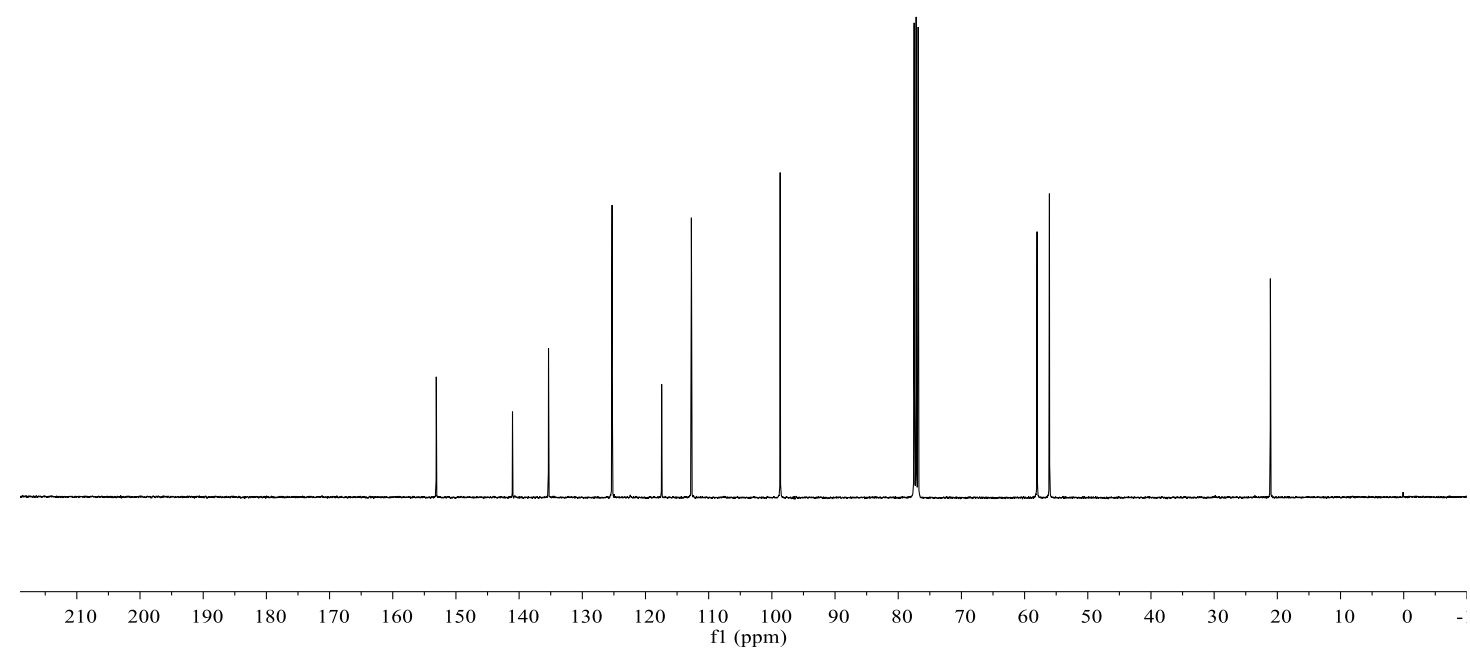




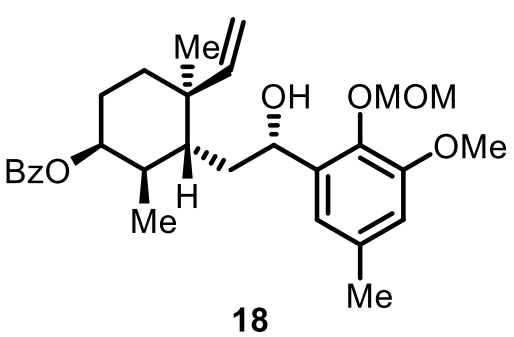

${ }^{1} \mathrm{H}$ NMR spectrum $\left(400 \mathrm{MHz}, \mathrm{CDCl}_{3}\right)$

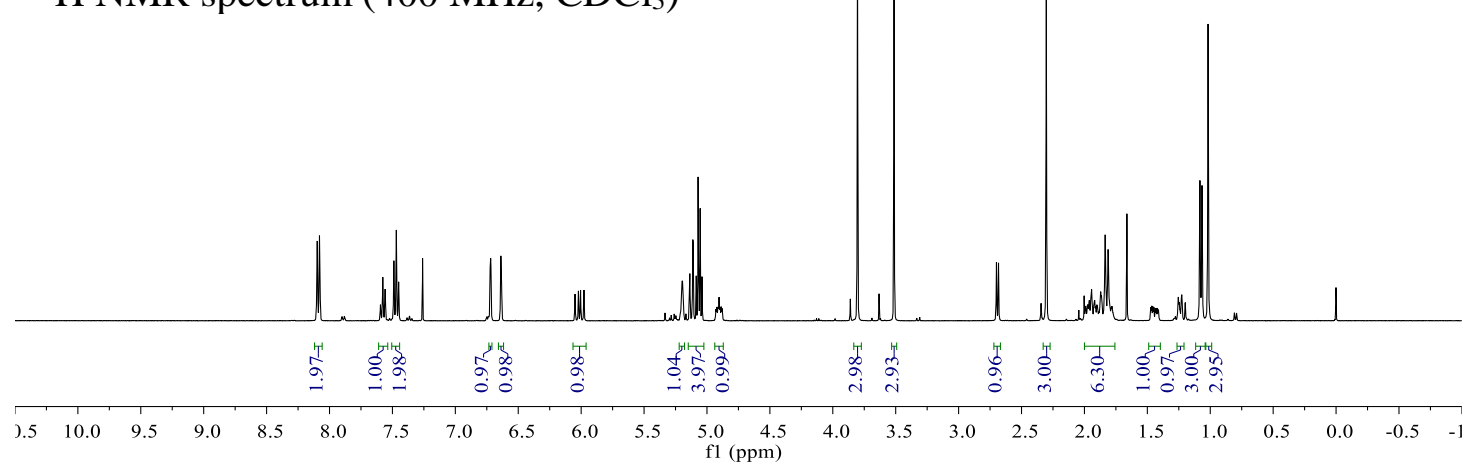

${ }^{13} \mathrm{C}$ NMR spectrum $\left(100 \mathrm{MHz}, \mathrm{CDCl}_{3}\right)$

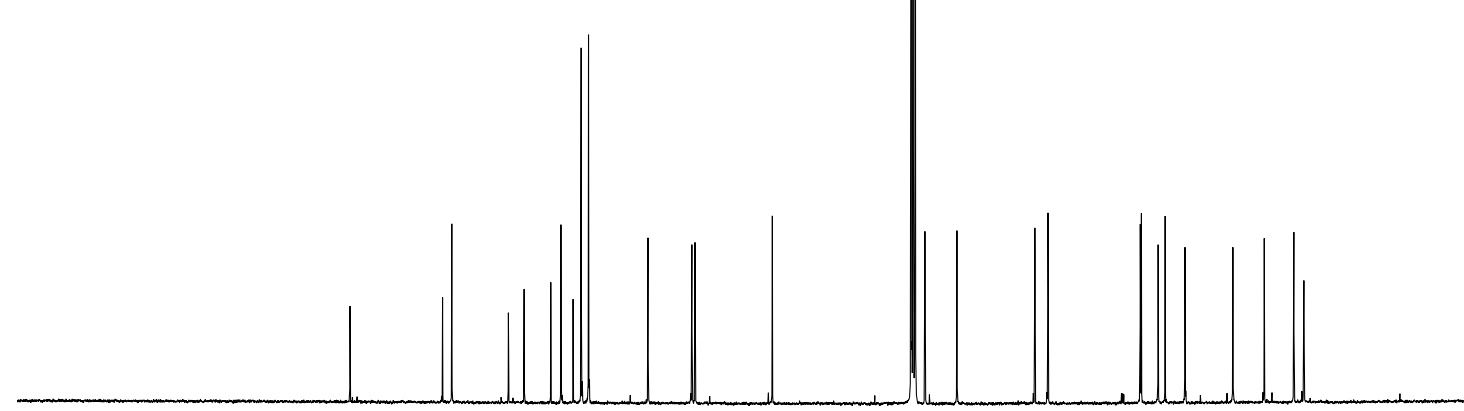

$\begin{array}{llllllllllllllllllllll}1 & 200 & 190 & 180 & 170 & 160 & 150 & 140 & 130 & 120 & \begin{array}{r}110 \\ \mathrm{f} 1(\mathrm{ppm})\end{array} & 90 & 80 & 70 & 60 & 50 & 40 & 30 & 20 & 10 & 0 & -\end{array}$ 


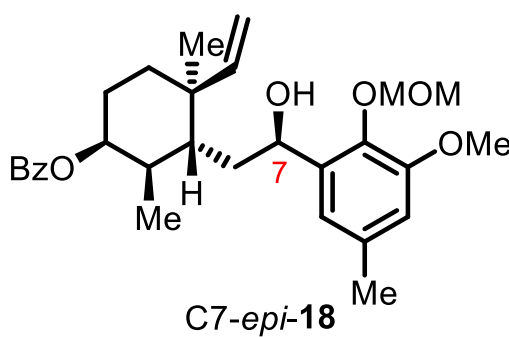

${ }^{1} \mathrm{H}$ NMR spectrum $\left(400 \mathrm{MHz}, \mathrm{CDCl}_{3}\right)$

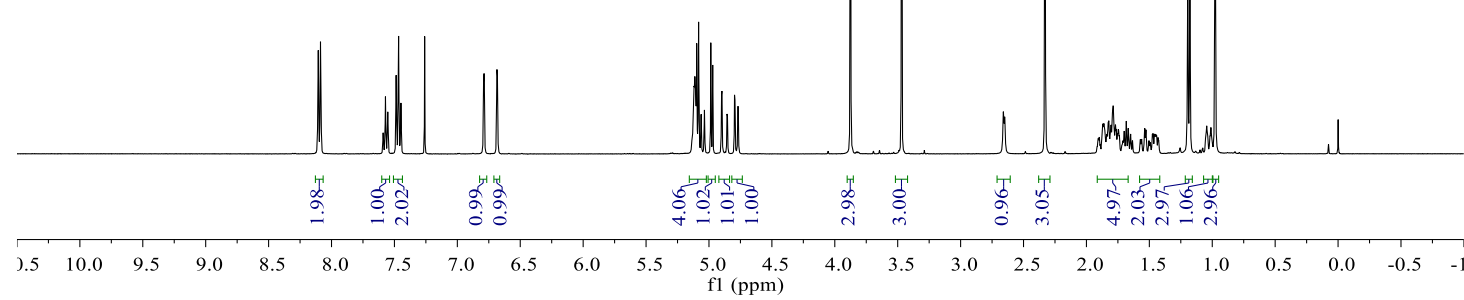

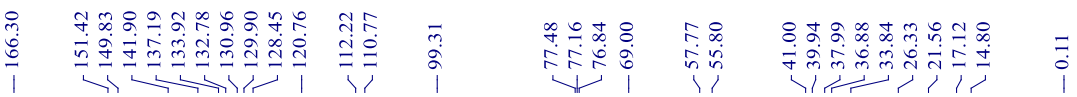

${ }^{13} \mathrm{C}$ NMR spectrum $\left(100 \mathrm{MHz}, \mathrm{CDCl}_{3}\right)$

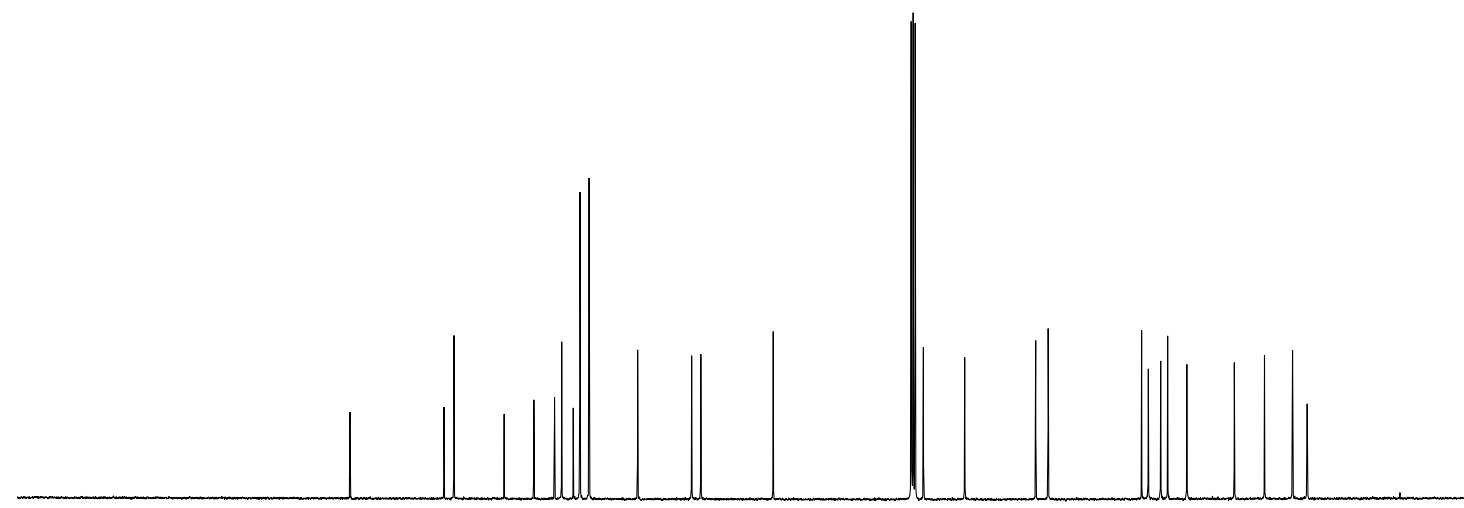

$\begin{array}{lllllllllllllllllllllll}210 & 200 & 190 & 180 & 170 & 160 & 150 & 140 & 130 & 120 & 110 & 100 & 90 & 80 & 70 & 60 & 50 & 40 & 30 & 20 & 10 & 0 & -\end{array}$ 


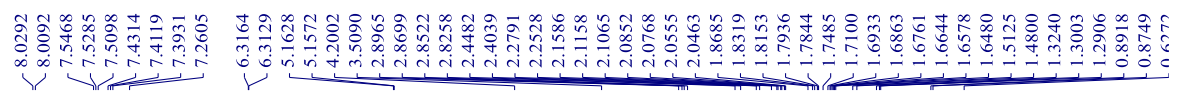

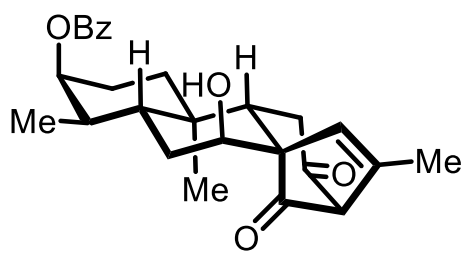

13

${ }^{1} \mathrm{H}$ NMR spectrum $\left(400 \mathrm{MHz}, \mathrm{CDCl}_{3}\right)$

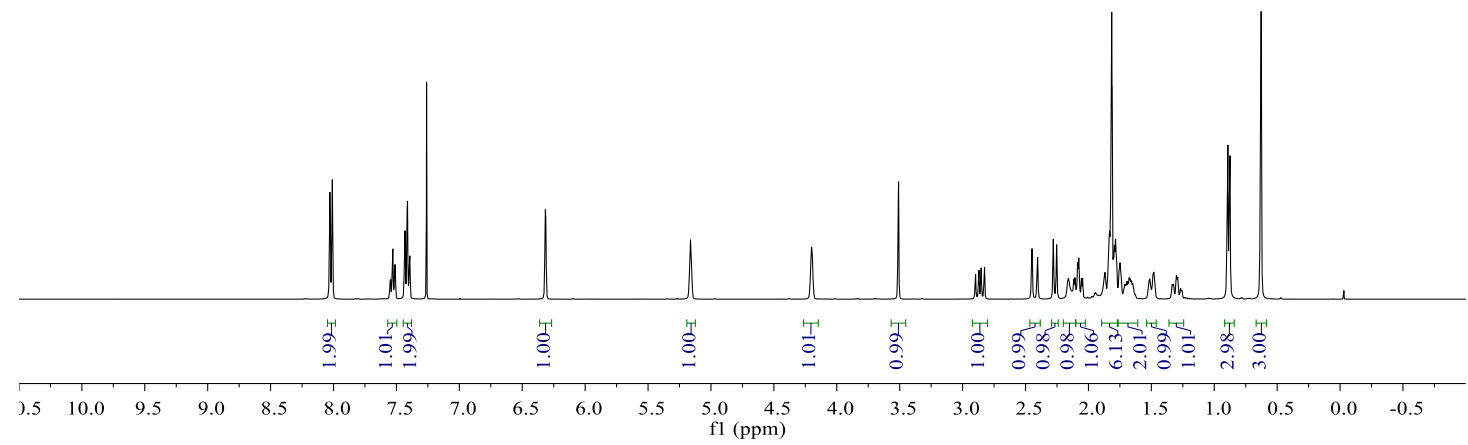

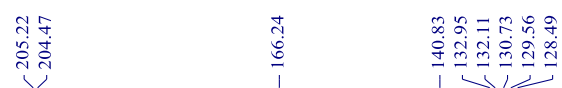

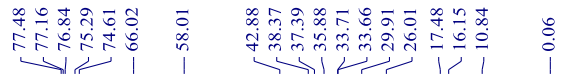

${ }^{13} \mathrm{C}$ NMR spectrum $\left(100 \mathrm{MHz}, \mathrm{CDCl}_{3}\right)$

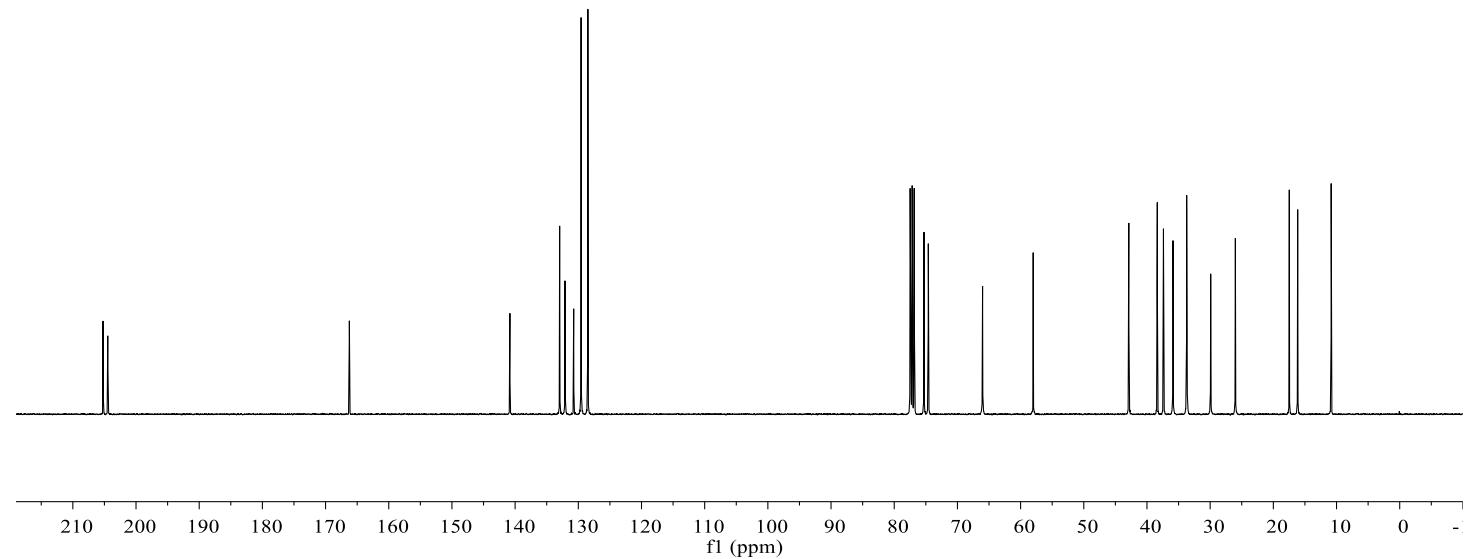




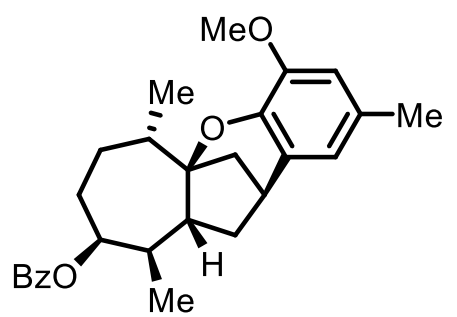

21

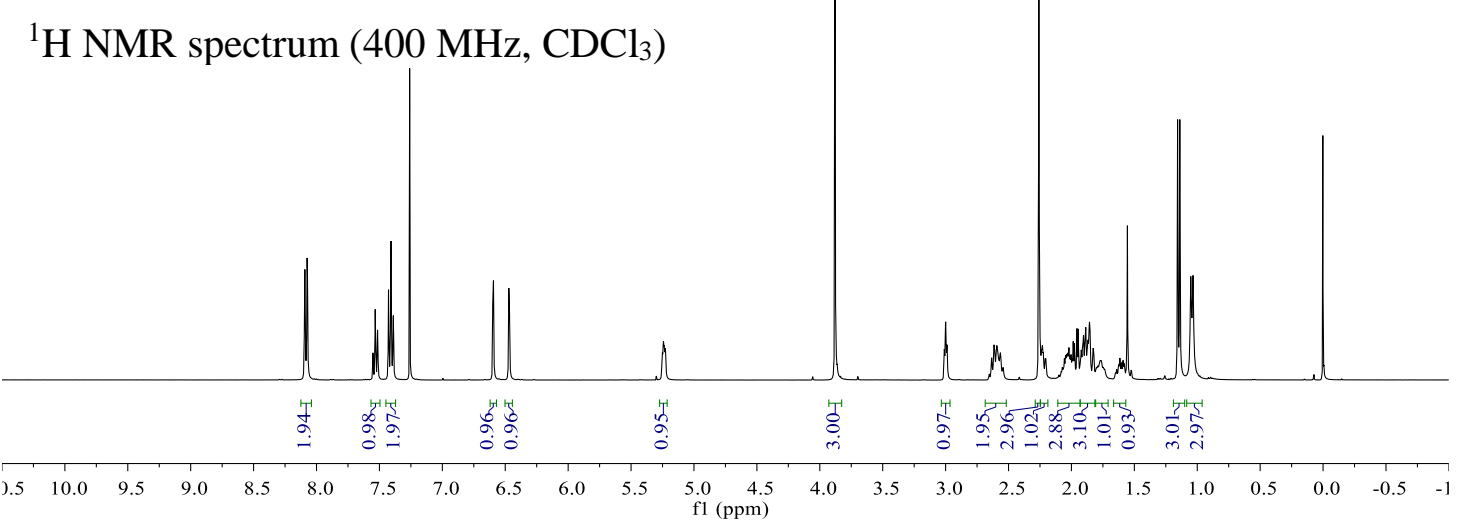

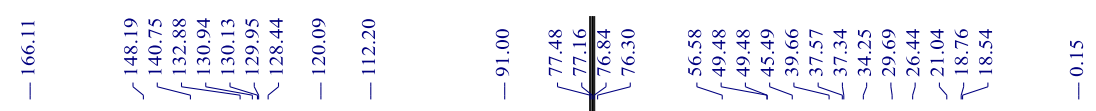

${ }^{13} \mathrm{C}$ NMR spectrum $\left(100 \mathrm{MHz}, \mathrm{CDCl}_{3}\right)$

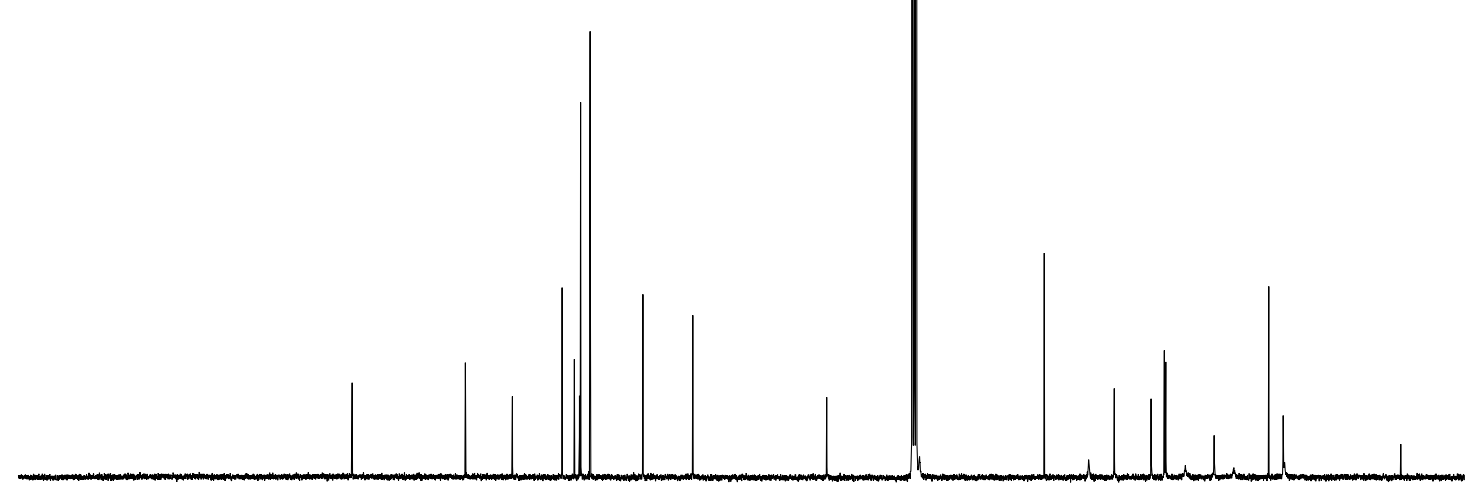

$\begin{array}{lllllllllllllllllllllll}210 & 200 & 190 & 180 & 170 & 160 & 150 & 140 & 130 & 120 & \begin{array}{c}110 \\ \mathrm{f}(\mathrm{ppm})\end{array} & 90 & 80 & 70 & 60 & 50 & 40 & 30 & 20 & 10 & 0 & -\end{array}$ 

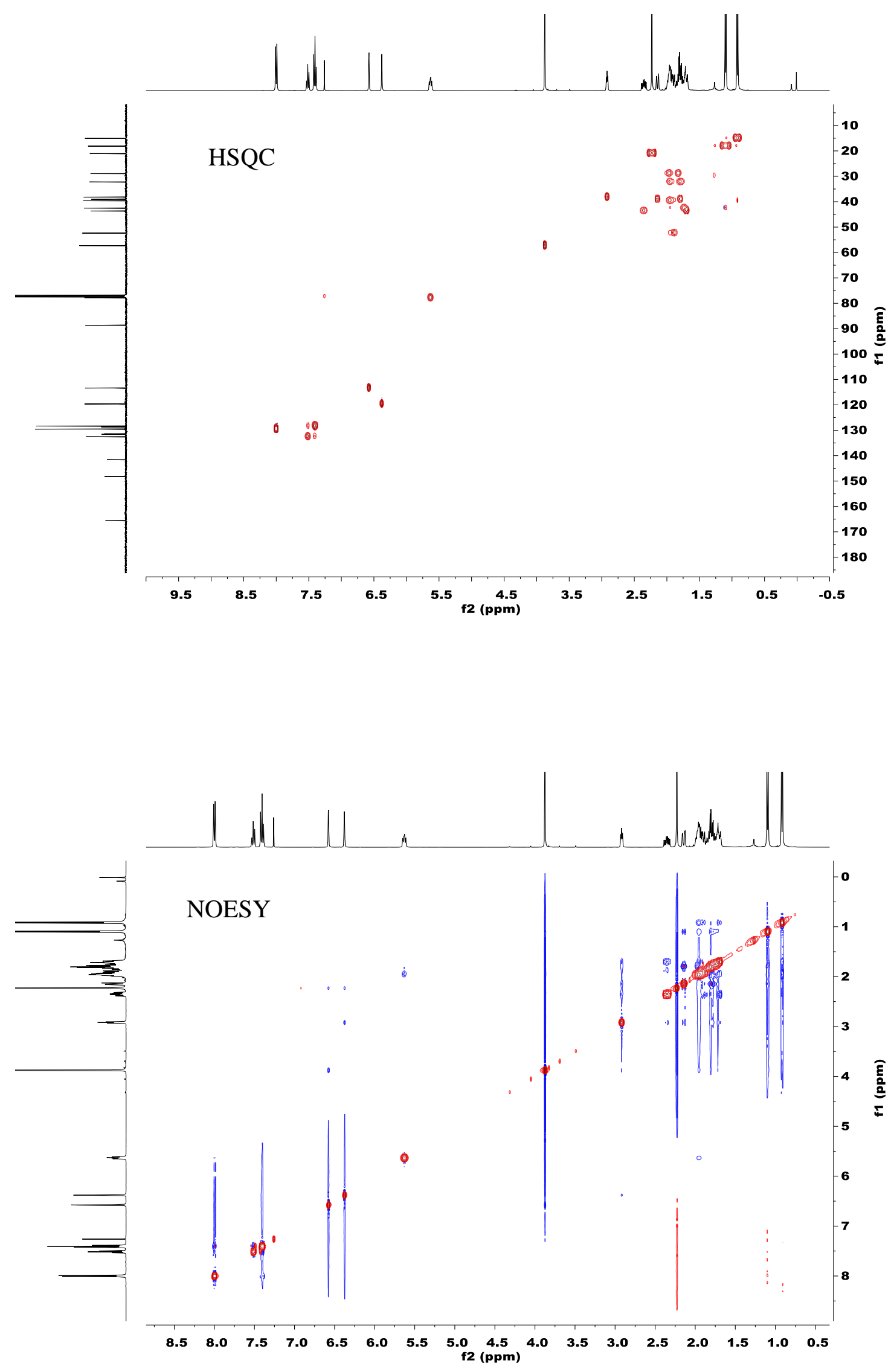


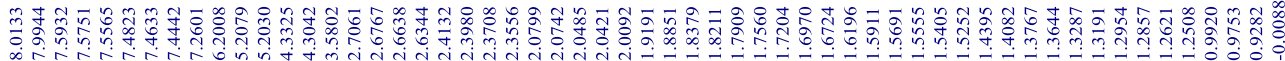

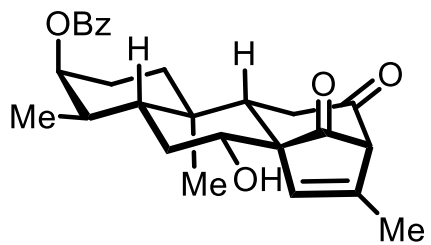

C7,C8,C13-triepi-13

${ }^{1} \mathrm{H}$ NMR spectrum $\left(400 \mathrm{MHz}, \mathrm{CDCl}_{3}\right)$

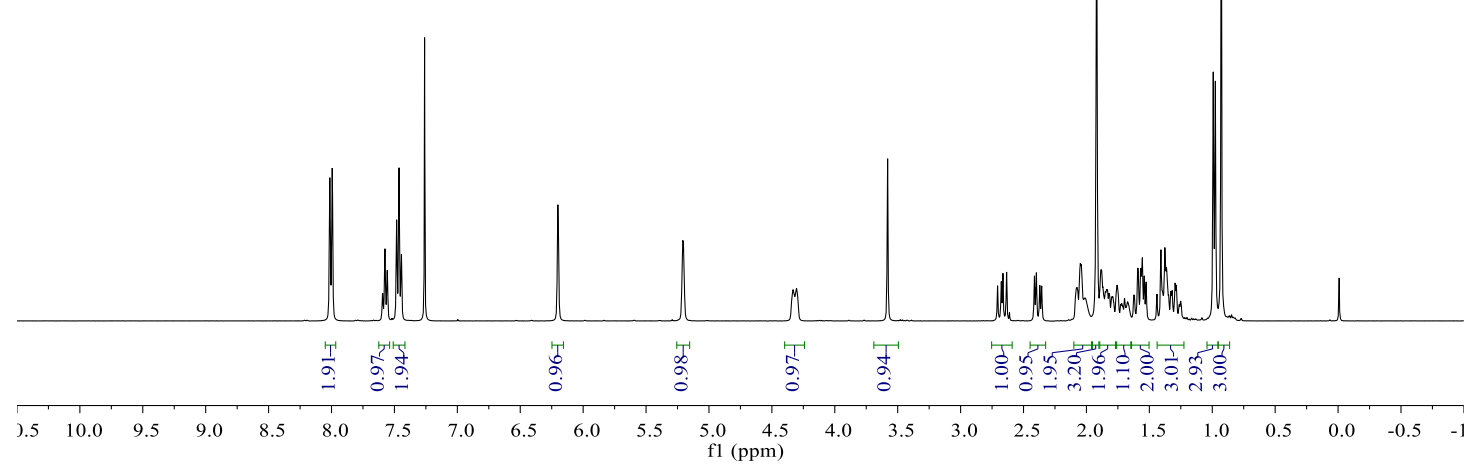

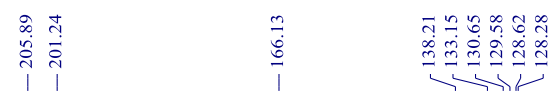

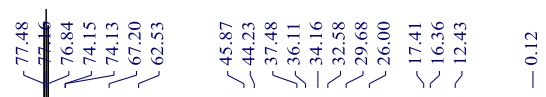

${ }^{13} \mathrm{C}$ NMR spectrum $\left(100 \mathrm{MHz}, \mathrm{CDCl}_{3}\right)$

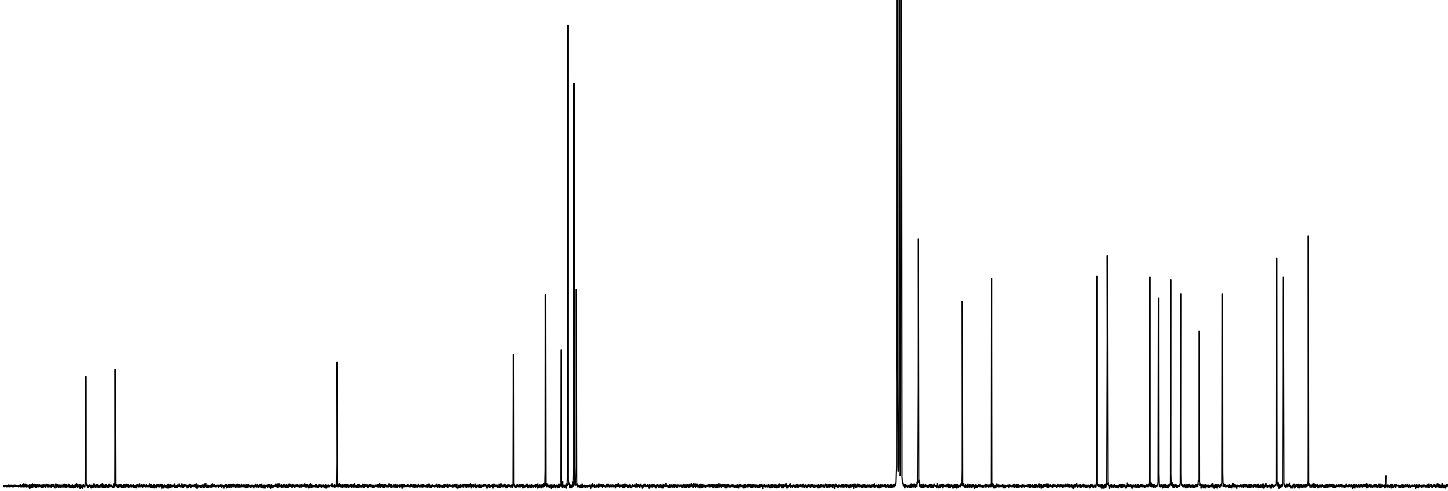

$\begin{array}{lllllllllllllllllllllll}210 & 200 & 190 & 180 & 170 & 160 & 150 & 140 & 130 & 120 & 110 & 100 & 90 & 80 & 70 & 60 & 50 & 40 & 30 & 20 & 10 & 0 & -.\end{array}$ 


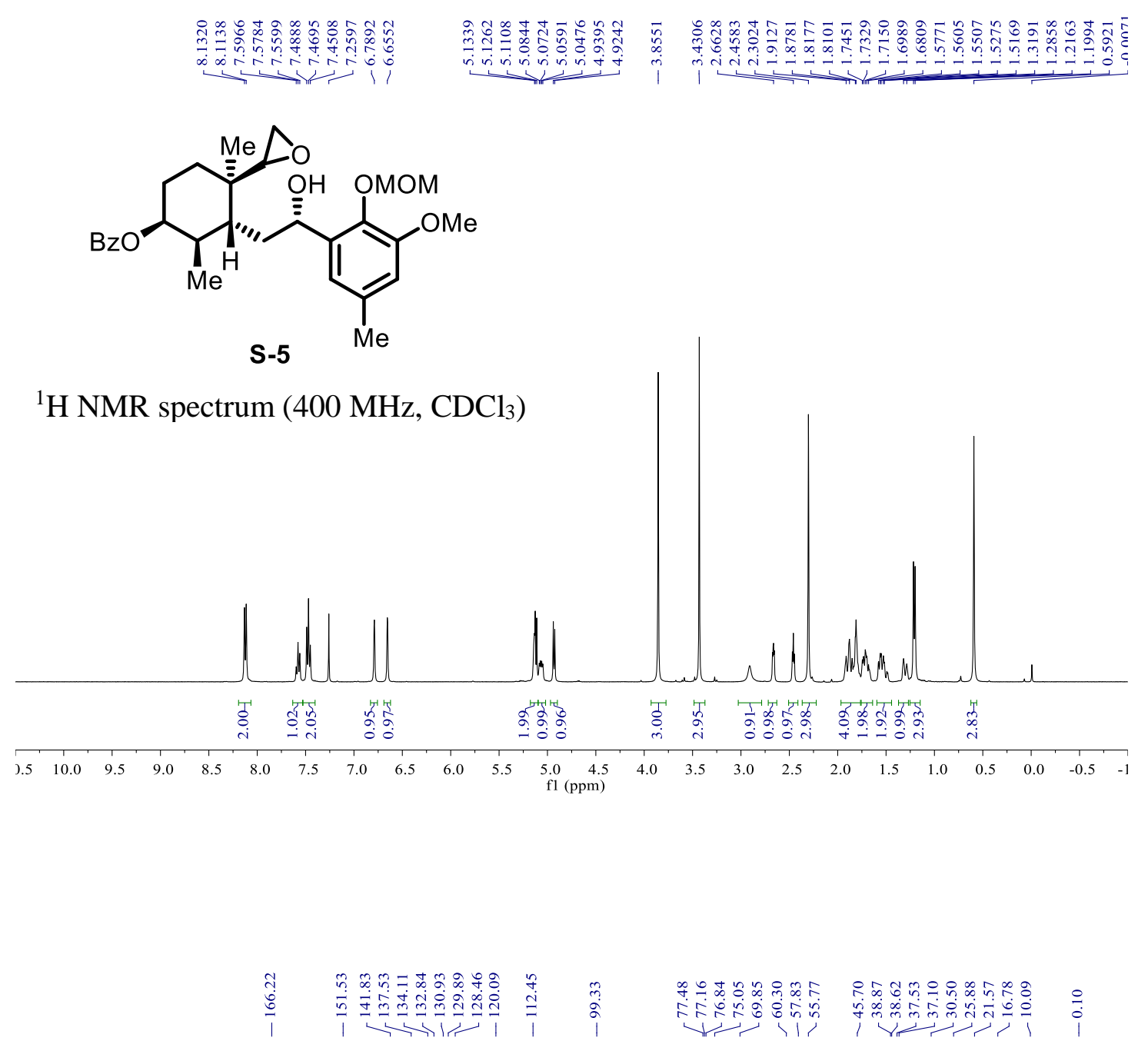

${ }^{13} \mathrm{C}$ NMR spectrum $\left(100 \mathrm{MHz}, \mathrm{CDCl}_{3}\right)$

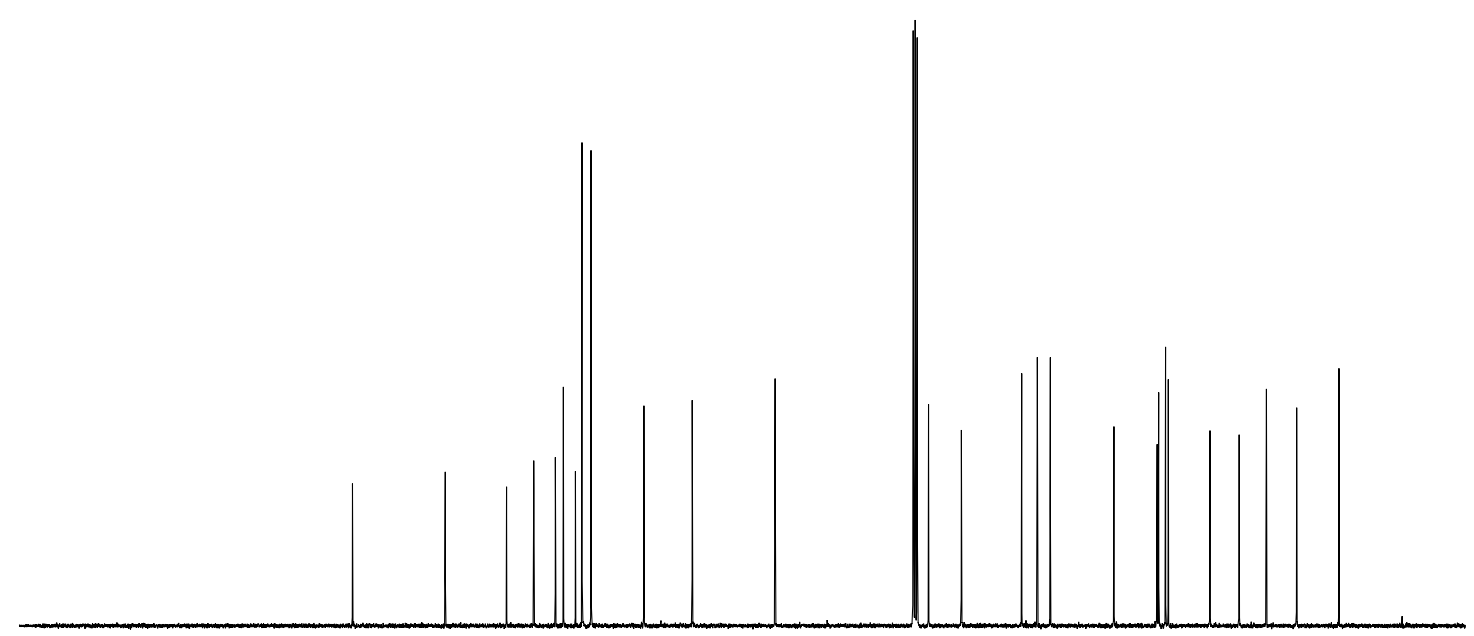

$\begin{array}{llllllllllllllllllllll}210 & 200 & 190 & 180 & 170 & 160 & 150 & 140 & 130 & 120 & \begin{array}{c}110 \\ \mathrm{f} 1(\mathrm{ppm})\end{array} & 90 & 80 & 70 & 60 & 50 & 40 & 30 & 20 & 10 & 0 & -\end{array}$ 


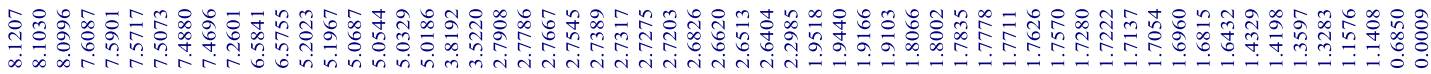

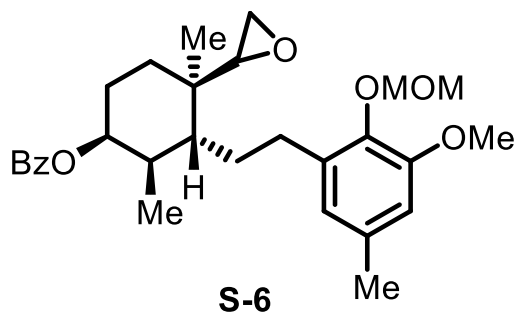

${ }^{1} \mathrm{H}$ NMR spectrum $\left(400 \mathrm{MHz}, \mathrm{CDCl}_{3}\right)$

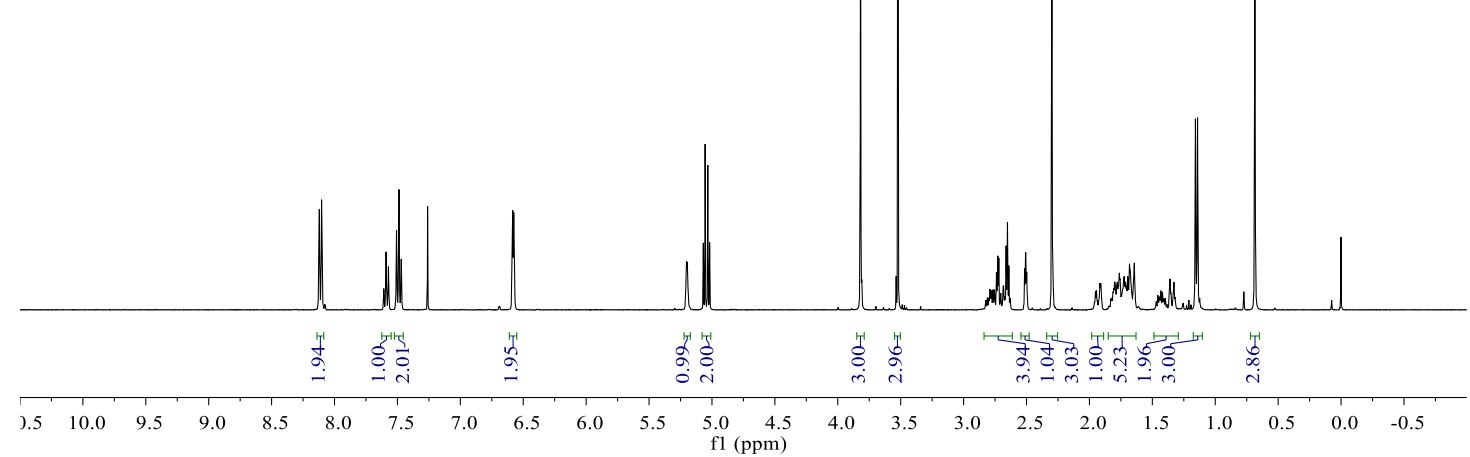

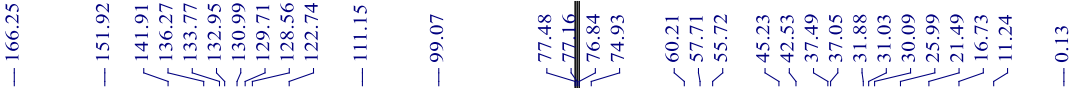

${ }^{13} \mathrm{C}$ NMR spectrum $\left(100 \mathrm{MHz}, \mathrm{CDCl}_{3}\right)$

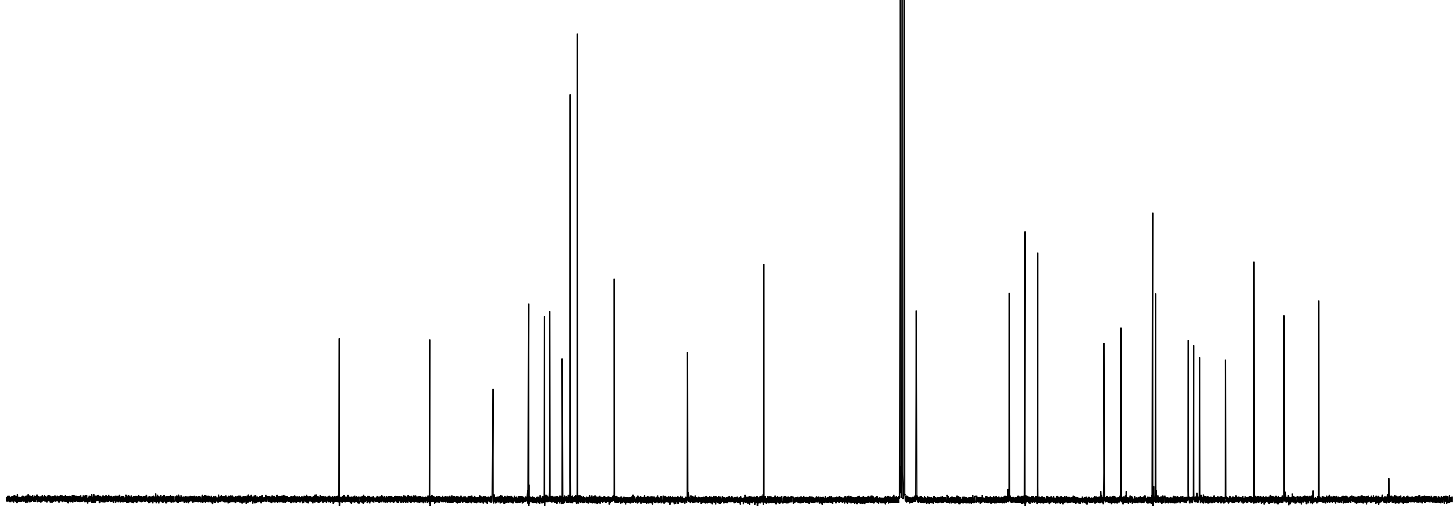

$\begin{array}{rlllllllllllllllllllll}210 & 200 & 190 & 180 & 170 & 160 & 150 & 140 & 130 & 120 & \begin{array}{r}110 \\ \mathrm{f} 1(\mathrm{ppm})\end{array} & 90 & 80 & 70 & 60 & 50 & 40 & 30 & 20 & 10 & 0 & -\end{array}$ 


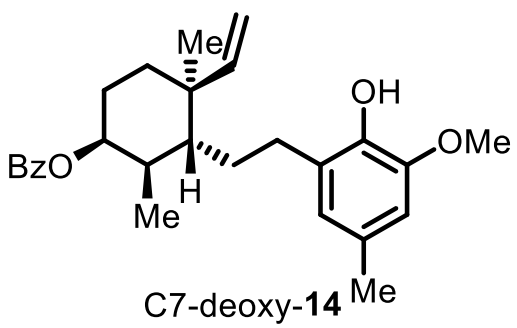

${ }^{1} \mathrm{H}$ NMR spectrum $\left(400 \mathrm{MHz}, \mathrm{CDCl}_{3}\right)$

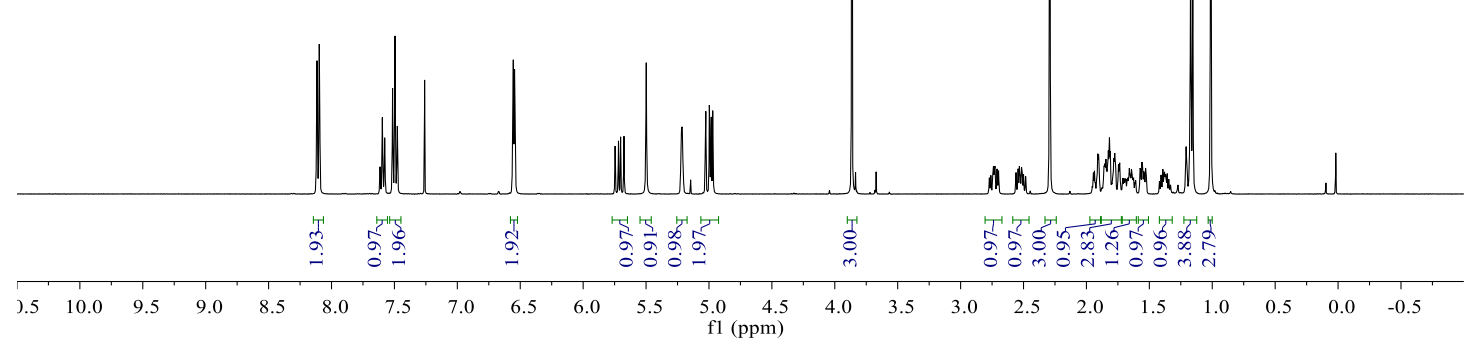

${ }^{13} \mathrm{C}$ NMR spectrum $\left(100 \mathrm{MHz}, \mathrm{CDCl}_{3}\right)$

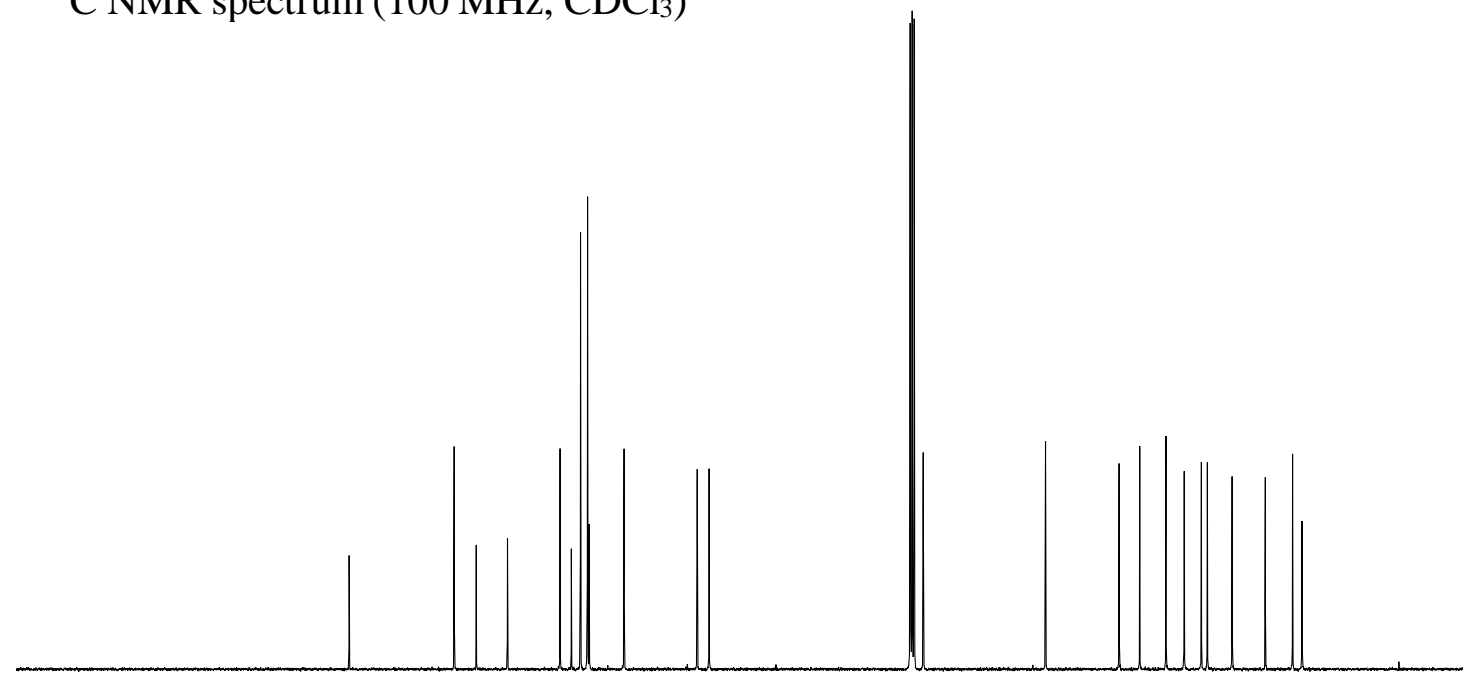

$\begin{array}{llllllllllllllllllllll}210 & 200 & 190 & 180 & 170 & 160 & 150 & 140 & 130 & 120 & \begin{array}{c}110 \\ \mathrm{f} 1(\mathrm{ppm})\end{array} & 100 & 80 & 70 & 60 & 50 & 40 & 30 & 20 & 10 & 0 & -\end{array}$ 


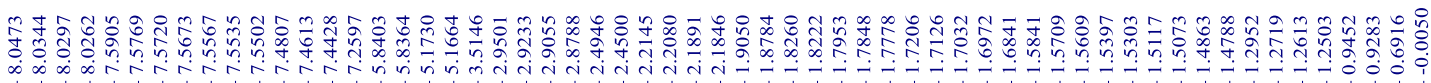

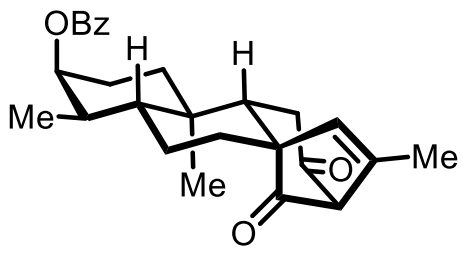

C7-deoxy-13

${ }^{1} \mathrm{H}$ NMR spectrum $\left(400 \mathrm{MHz}, \mathrm{CDCl}_{3}\right)$

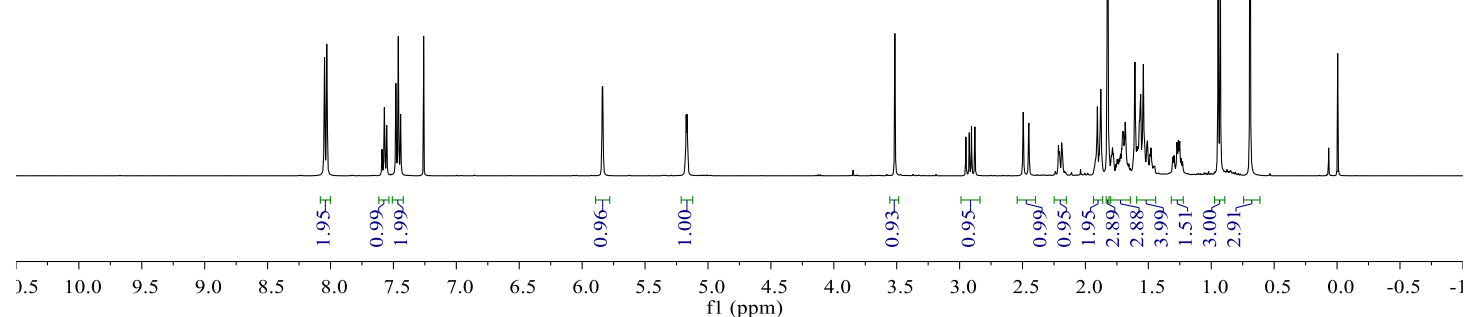

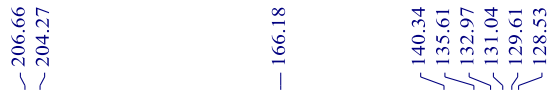

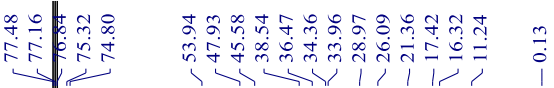

${ }^{13} \mathrm{C}$ NMR spectrum $\left(100 \mathrm{MHz}, \mathrm{CDCl}_{3}\right)$

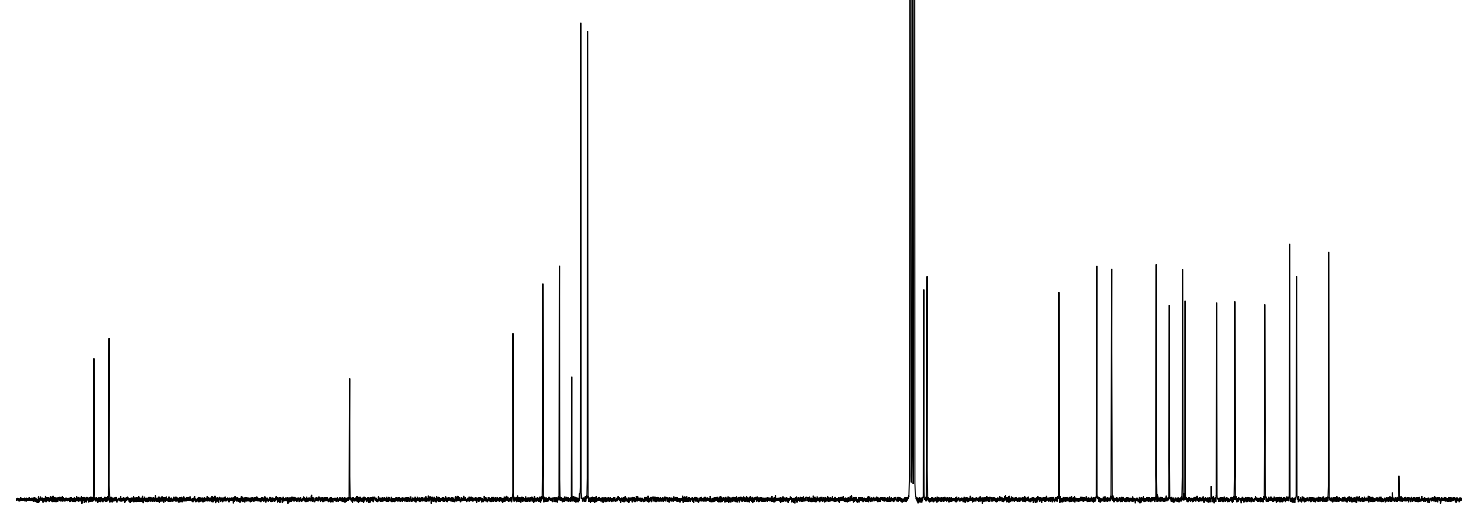

$\begin{array}{llllllllllllllllllllll}210 & 200 & 190 & 180 & 170 & 160 & 150 & 140 & 130 & 120 & \begin{array}{c}110 \\ \mathrm{f} 1(\mathrm{ppm})\end{array} & 100 & 80 & 70 & 60 & 50 & 40 & 30 & 20 & 10 & 0 & -\end{array}$ 


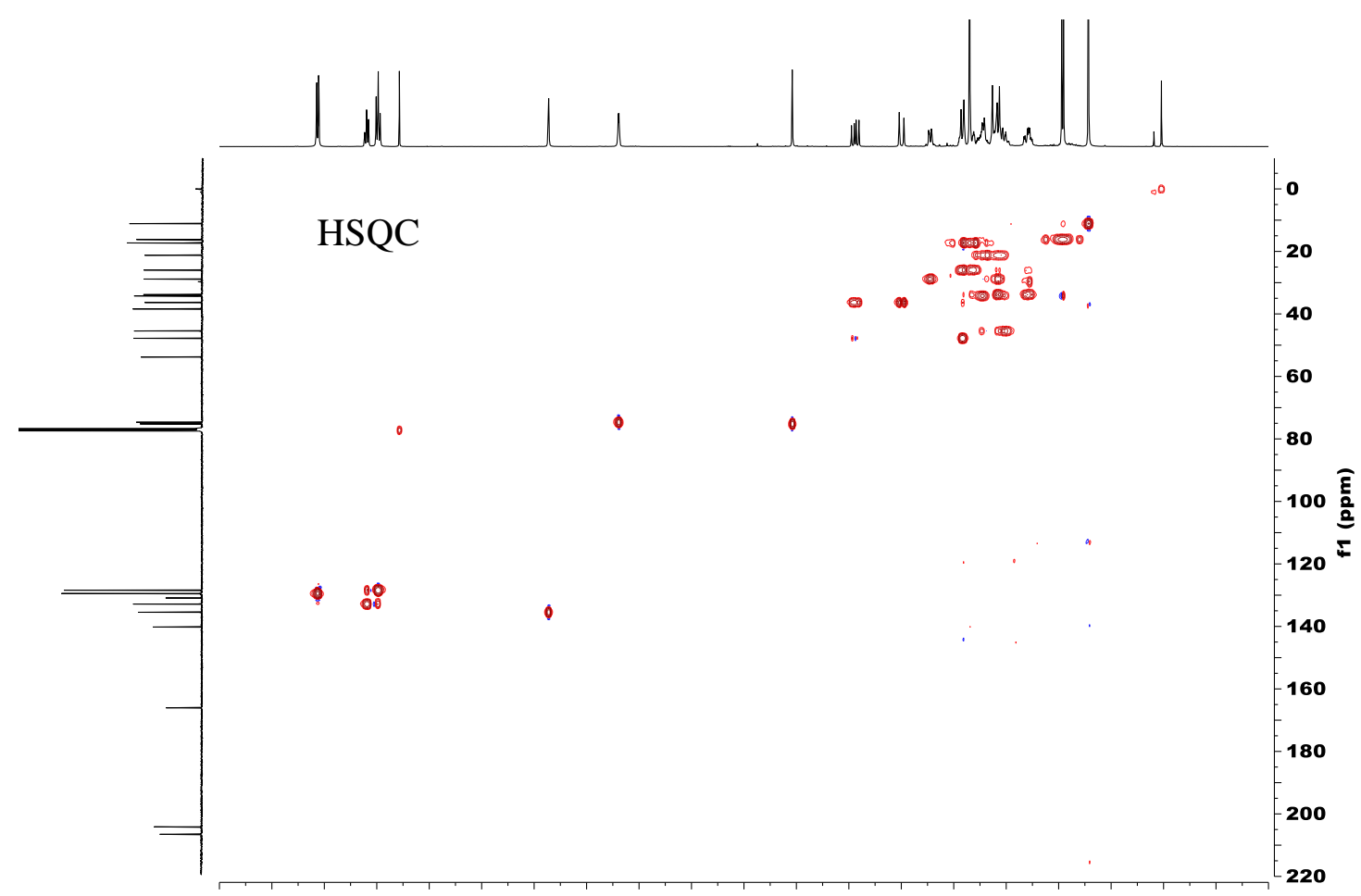

$\begin{array}{lllllllllllllllllllllllllllll}9.0 & 8.5 & 8.0 & 7.5 & 7.0 & 6.5 & 6.0 & 5.5 & 5.0 & 4.5 & 4.0 & 3.5 & 3.0 & 2.5 & 2.0 & 1.5 & 1.0 & 0.5 & 0.0 & -0.5 & -1.0\end{array}$

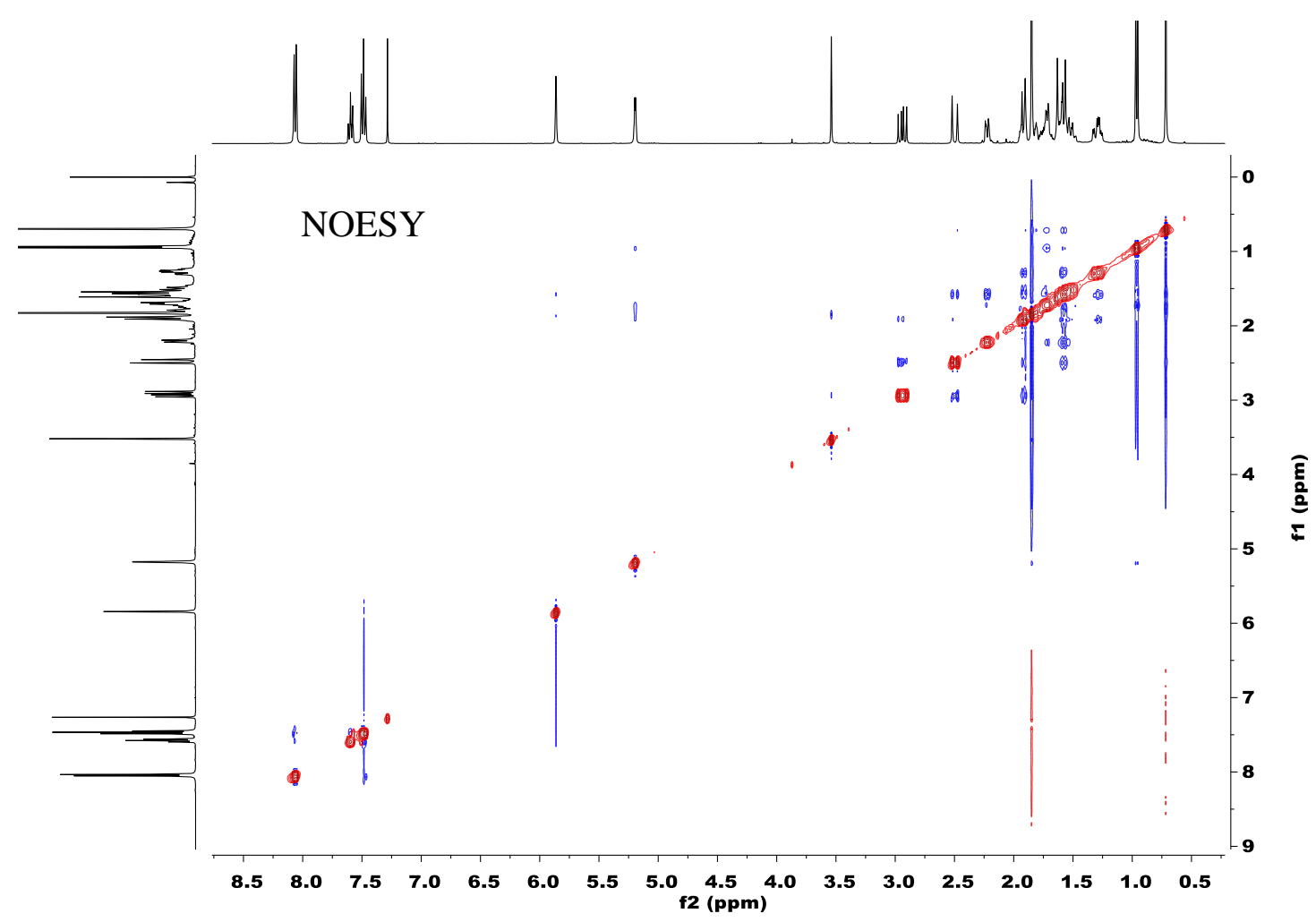



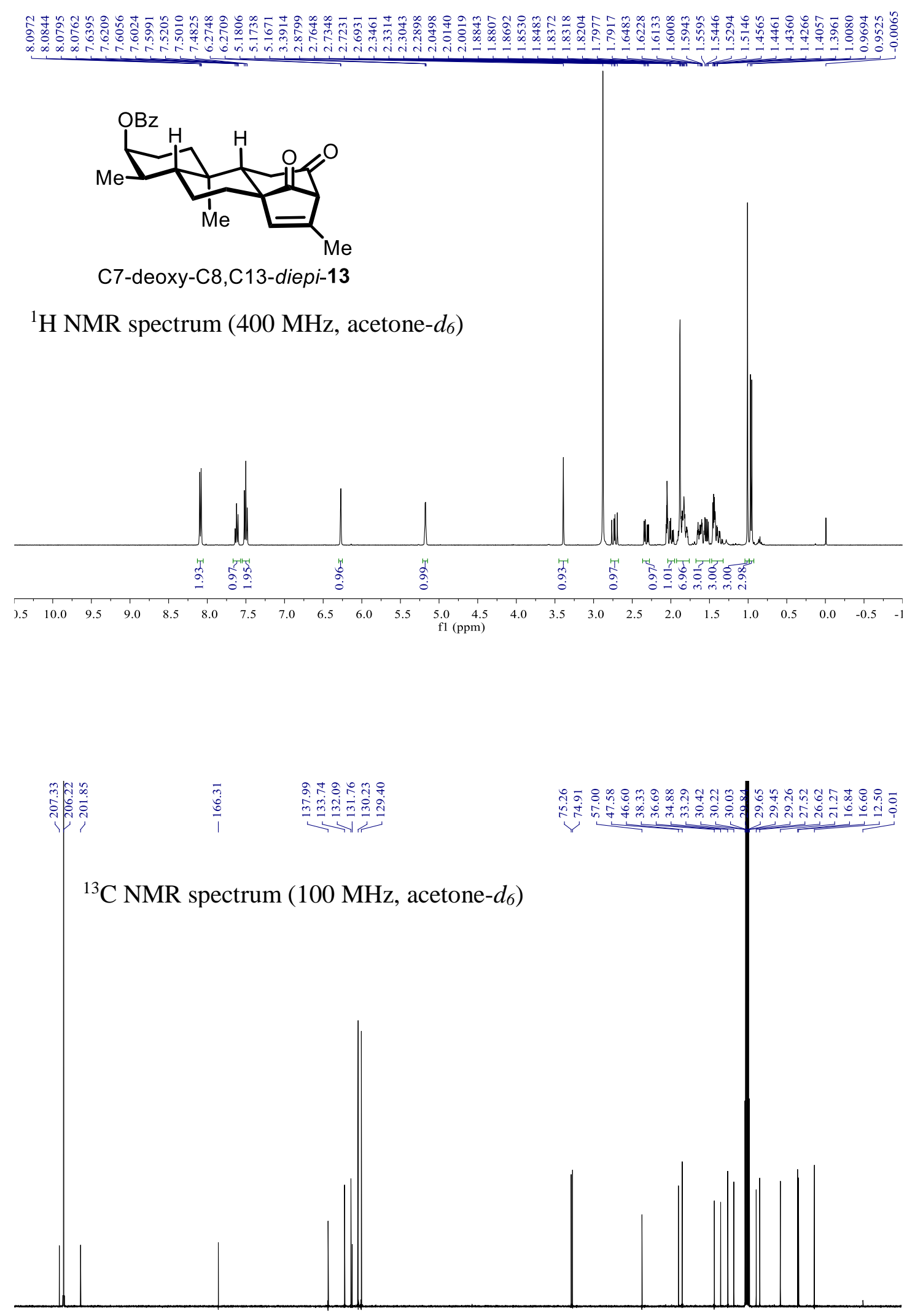

$\begin{array}{lllllllllllllllllllllllll}210 & 200 & 190 & 180 & 170 & 160 & 150 & 140 & 130 & 120 & 110 & 100 & 90 & 80 & 70 & 60 & 50 & 40 & 30 & 20 & 10 & 0 & -\end{array}$ 

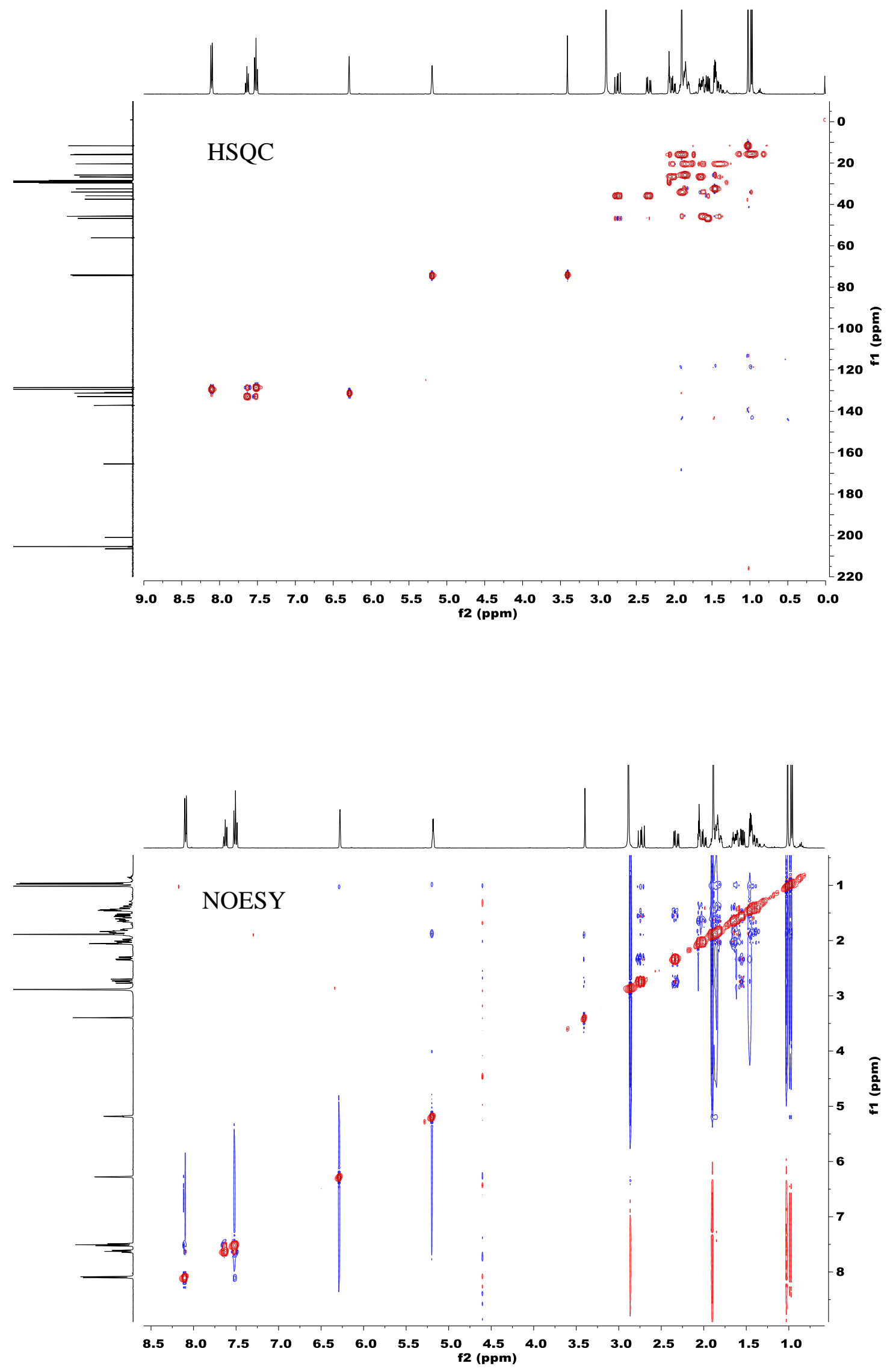


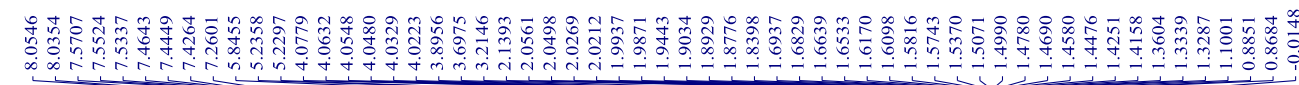

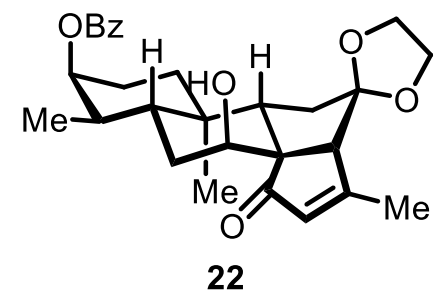

${ }^{1} \mathrm{H}$ NMR spectrum $\left(400 \mathrm{MHz}, \mathrm{CDCl}_{3}\right)$
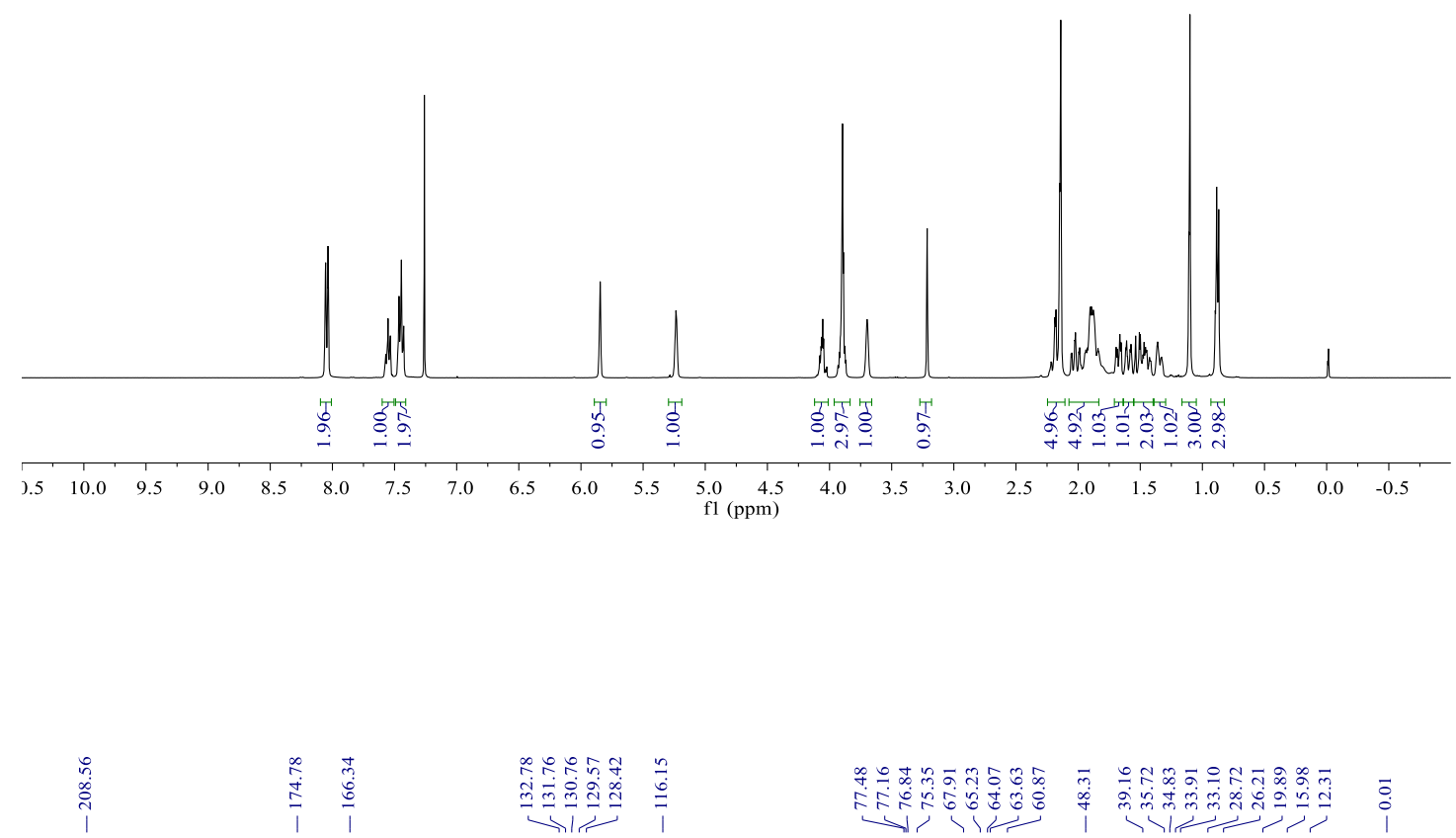

${ }^{13} \mathrm{C}$ NMR spectrum $\left(100 \mathrm{MHz}, \mathrm{CDCl}_{3}\right)$

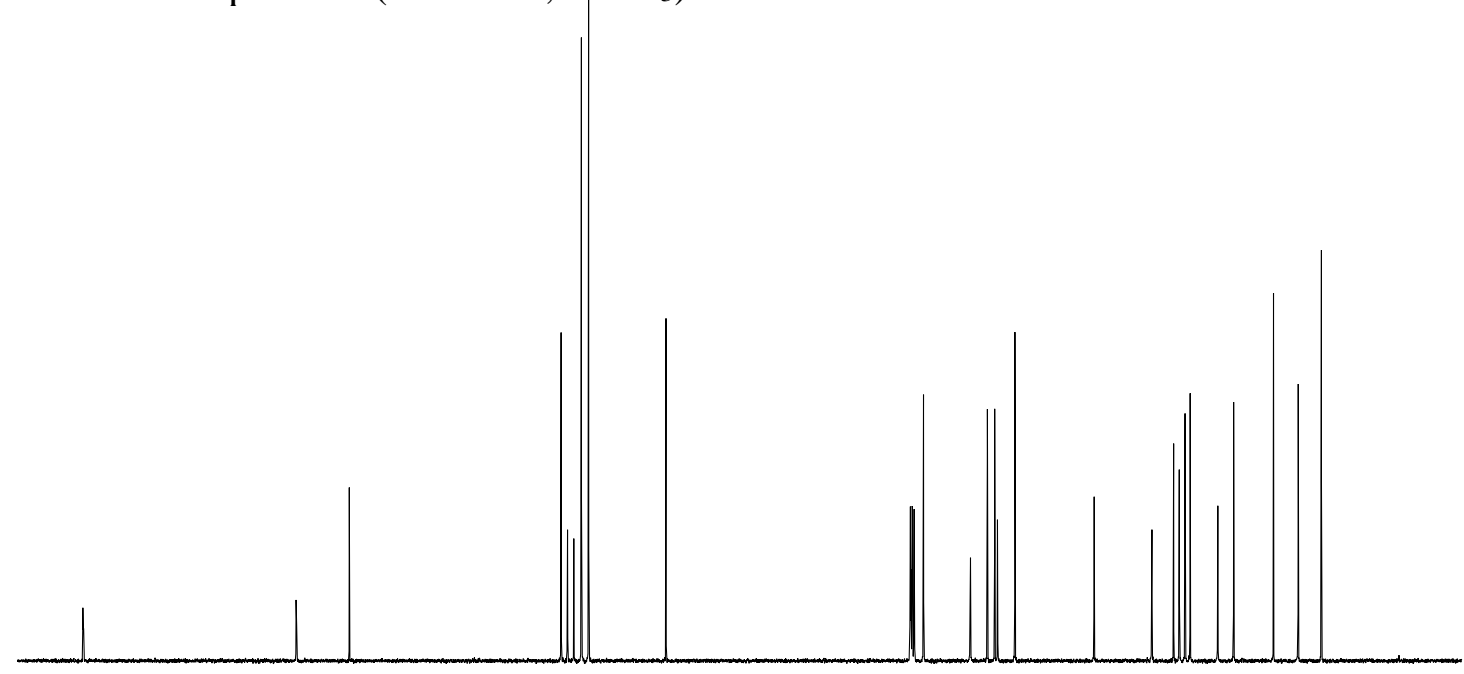

$\begin{array}{rlllllllllll}210 & 200 & 190 & 180 & 170 & 160 & 150 & 140 & 130 & 120 & 110 & 100 \\ \text { f1 (ppm) }\end{array}$ 


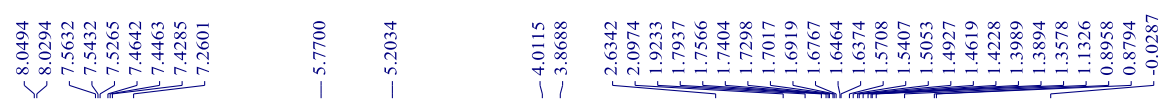

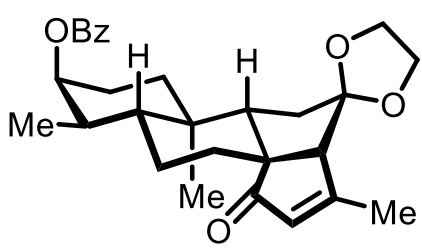

23

${ }^{1} \mathrm{H}$ NMR spectrum $\left(400 \mathrm{MHz}, \mathrm{CDCl}_{3}\right)$

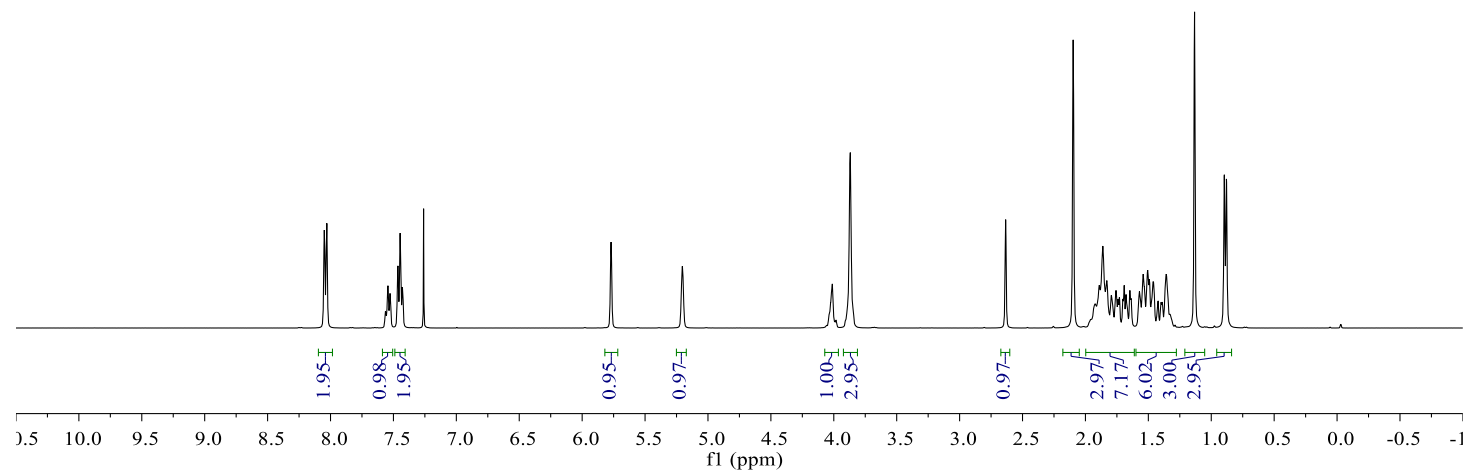

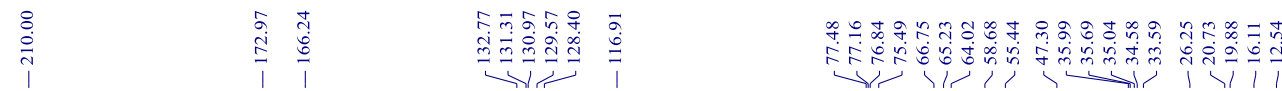

${ }^{13} \mathrm{C}$ NMR spectrum $\left(100 \mathrm{MHz}, \mathrm{CDCl}_{3}\right)$

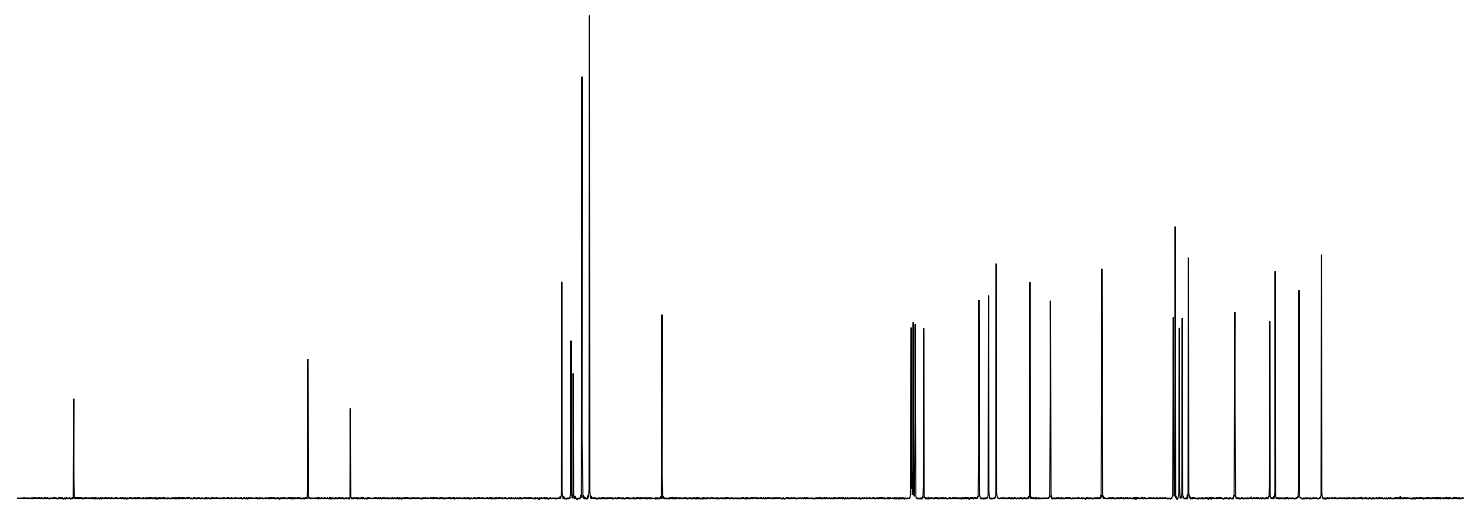

$\begin{array}{llllllllllllllllllllll}210 & 200 & 190 & 180 & 170 & 160 & 150 & 140 & 130 & 120 & \begin{array}{c}110 \\ \mathrm{f}(\mathrm{ppm})\end{array} & 90 & 80 & 70 & 60 & 50 & 40 & 30 & 20 & 10 & 0 & -\end{array}$ 


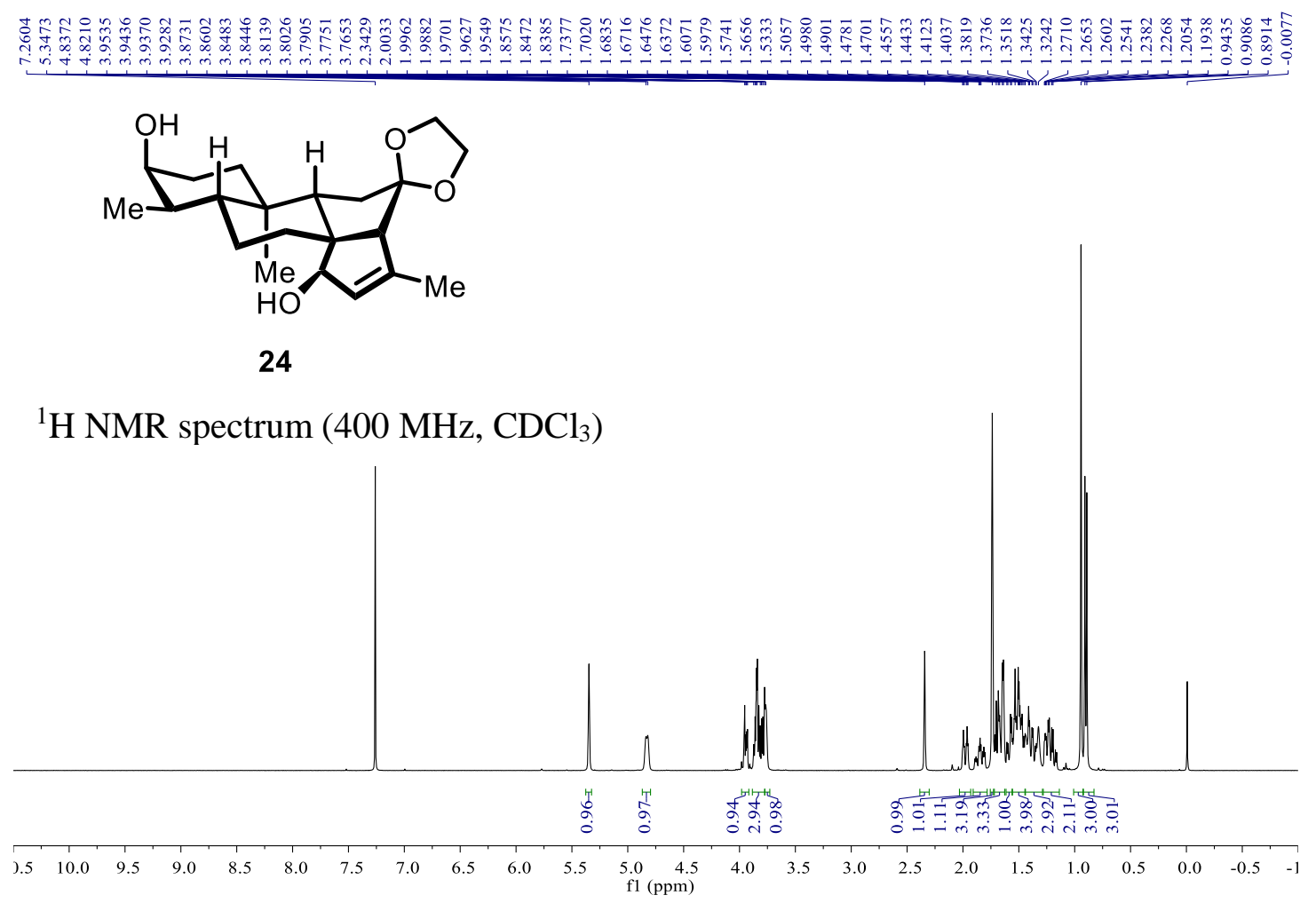

${ }^{13} \mathrm{C}$ NMR spectrum $\left(100 \mathrm{MHz}, \mathrm{CDCl}_{3}\right)$
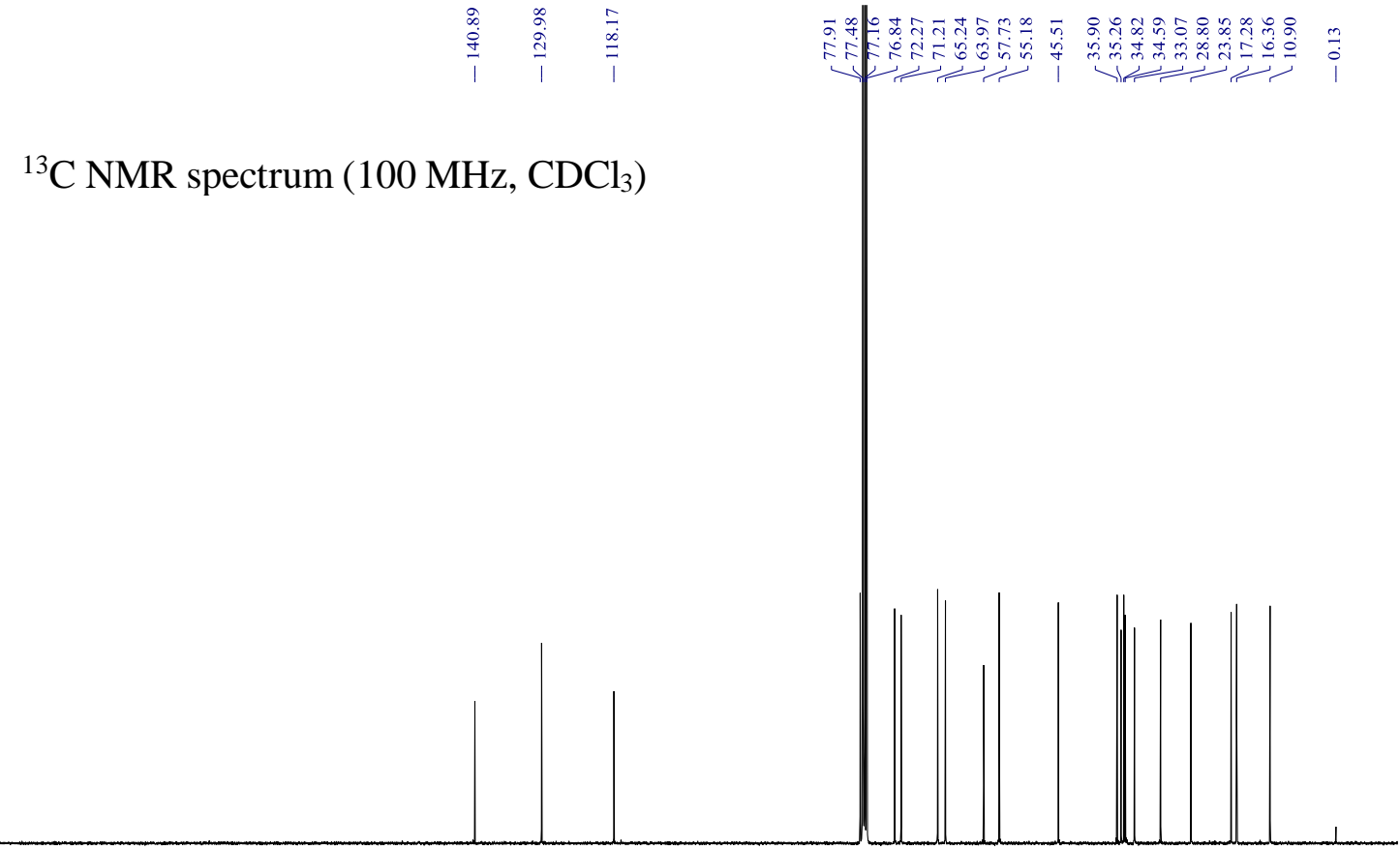

$\begin{array}{llllllllllllllllllllllll}1 & 210 & 200 & 190 & 180 & 170 & 160 & 150 & 140 & 130 & 120 & \begin{array}{c}110 \\ \mathrm{f}(\mathrm{ppm})\end{array} & 90 & 80 & 70 & 60 & 50 & 40 & 30 & 20 & 10 & 0 & -\end{array}$ 


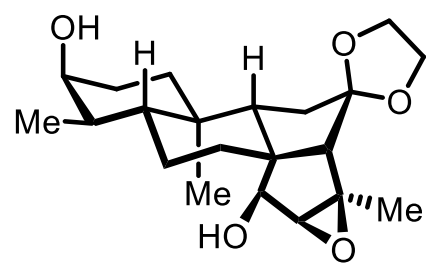

25

${ }^{1} \mathrm{H}$ NMR spectrum $\left(400 \mathrm{MHz}, \mathrm{CDCl}_{3}\right)$

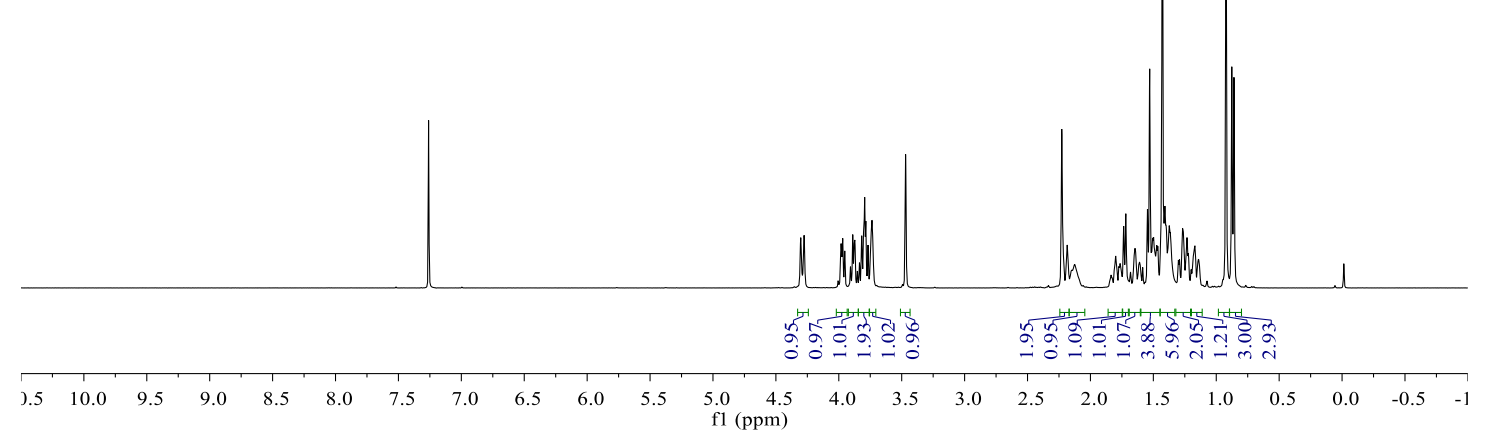

${ }^{13} \mathrm{C}$ NMR spectrum $\left(100 \mathrm{MHz}, \mathrm{CDCl}_{3}\right)$
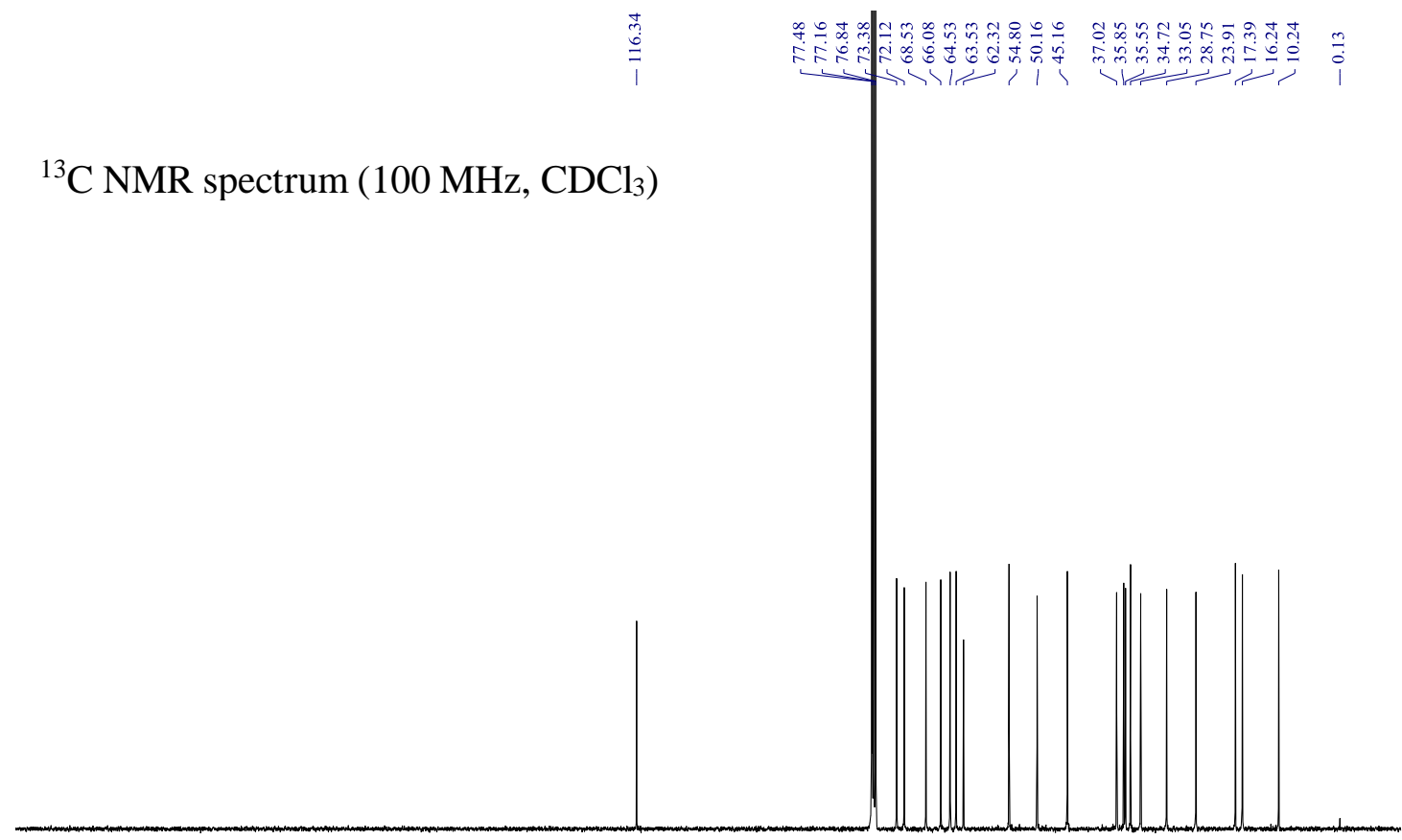

$\begin{array}{lllllllllllllllllllllll}210 & 200 & 190 & 180 & 170 & 160 & 150 & 140 & 130 & 120 & 110 & 100 & 90 & 80 & 70 & 60 & 50 & 40 & 30 & 20 & 10 & 0 & -\end{array}$ 


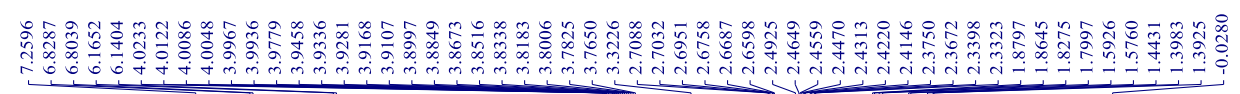

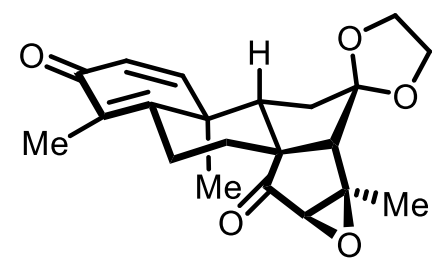

26

${ }^{1} \mathrm{H}$ NMR spectrum $\left(400 \mathrm{MHz}, \mathrm{CDCl}_{3}\right)$

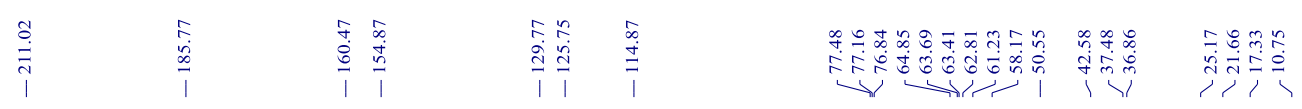

${ }^{13} \mathrm{C}$ NMR spectrum $\left(100 \mathrm{MHz}, \mathrm{CDCl}_{3}\right)$

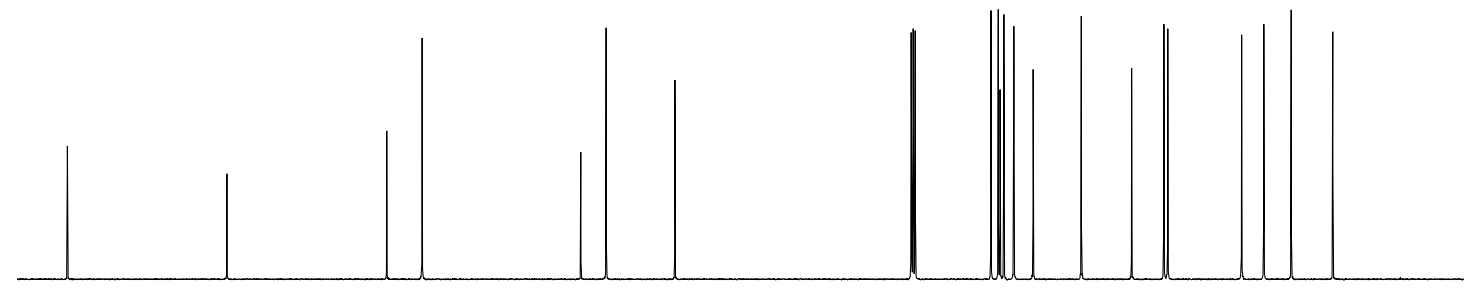

$\begin{array}{rlllllllllllllllllllll}210 & 200 & 190 & 180 & 170 & 160 & 150 & 140 & 130 & 120 & \begin{array}{r}110 \\ \mathrm{f} 1(\mathrm{ppm})\end{array} & 100 & 80 & 70 & 60 & 50 & 40 & 30 & 20 & 10 & 0 & -\end{array}$ 


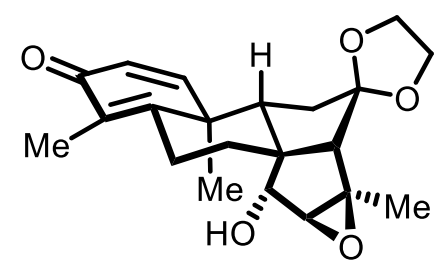

27

${ }^{1} \mathrm{H}$ NMR spectrum (400 MHz, $\mathrm{CDCl}_{3}$ )

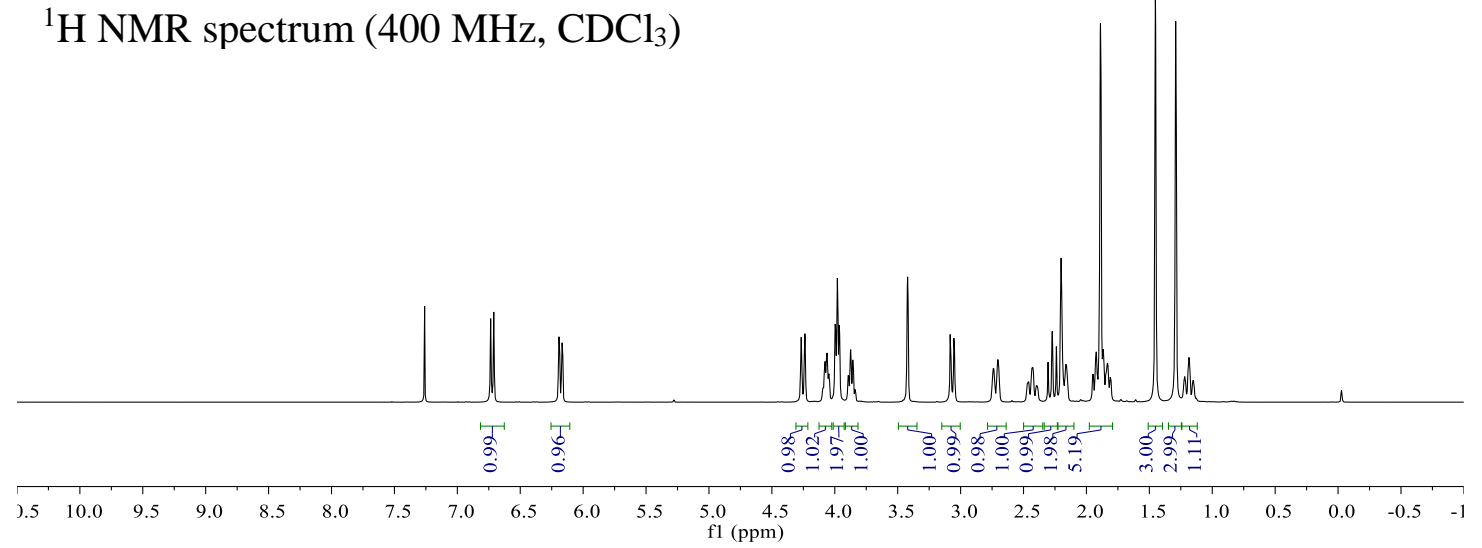

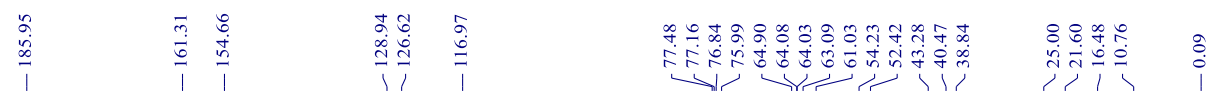

${ }^{13} \mathrm{C}$ NMR spectrum $\left(100 \mathrm{MHz}, \mathrm{CDCl}_{3}\right)$

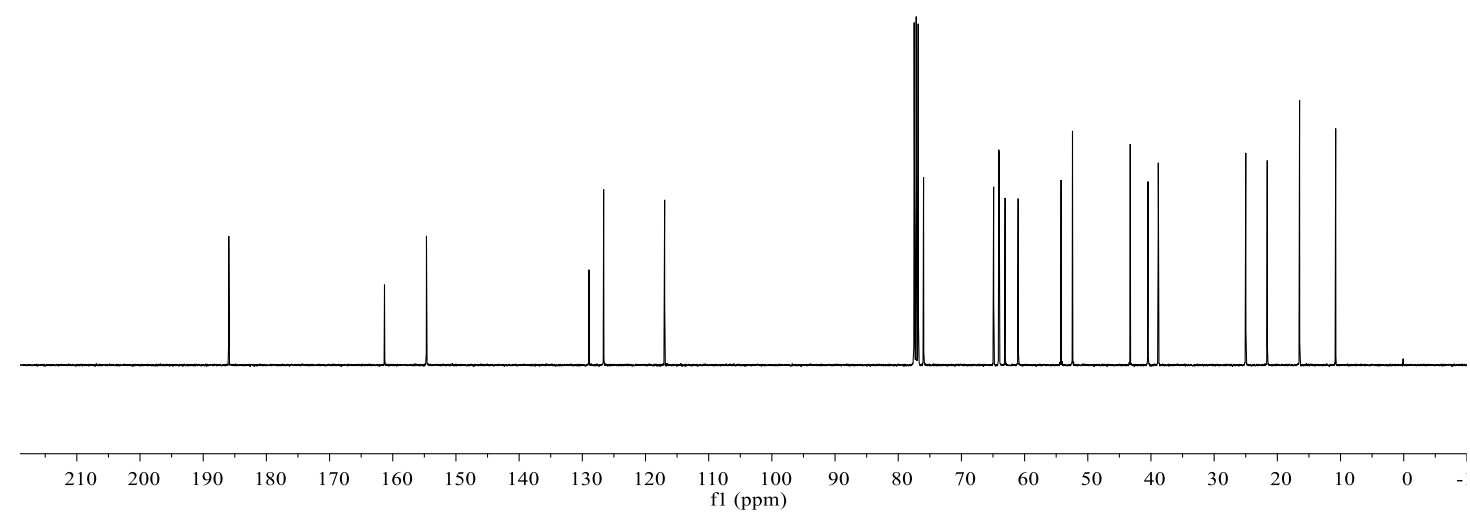




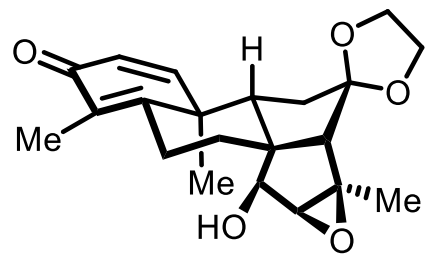

C14-epi-27

${ }^{1} \mathrm{H}$ NMR spectrum $\left(400 \mathrm{MHz}, \mathrm{CDCl}_{3}\right)$

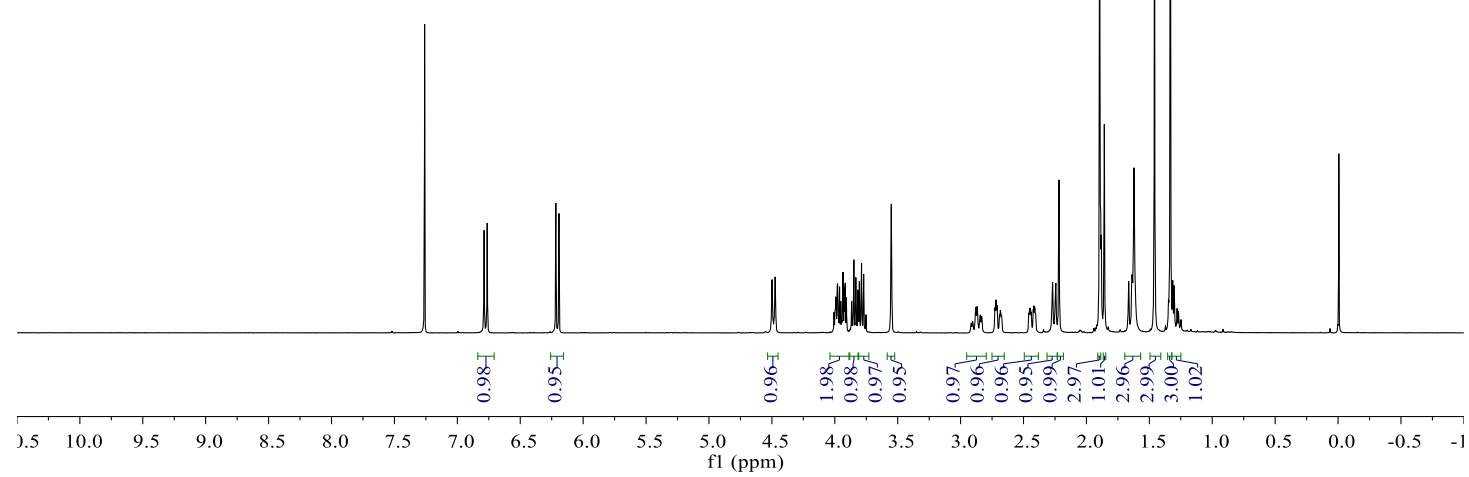

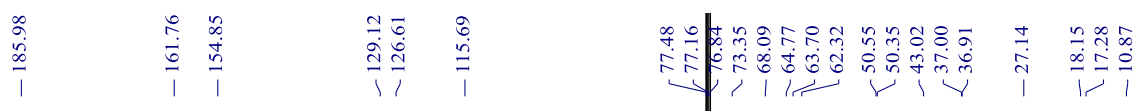

${ }^{13} \mathrm{C}$ NMR spectrum $\left(100 \mathrm{MHz}, \mathrm{CDCl}_{3}\right)$

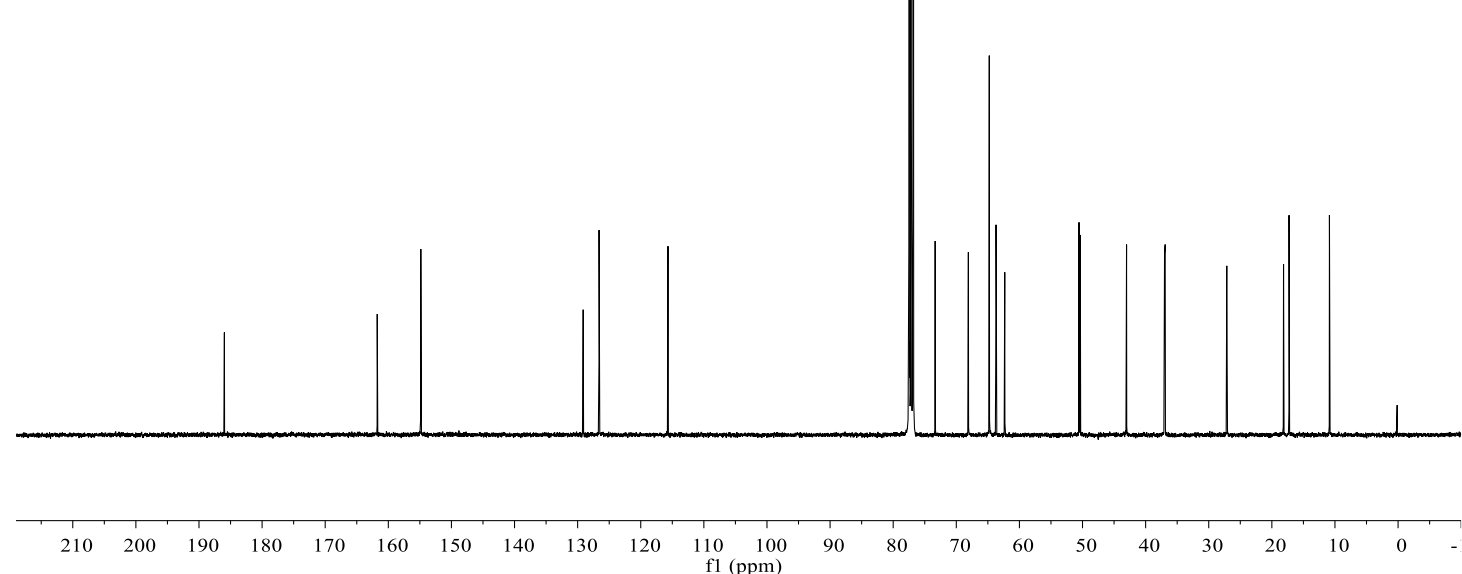




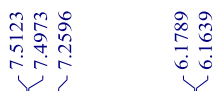

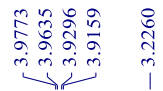

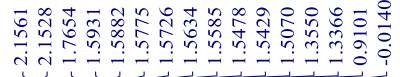

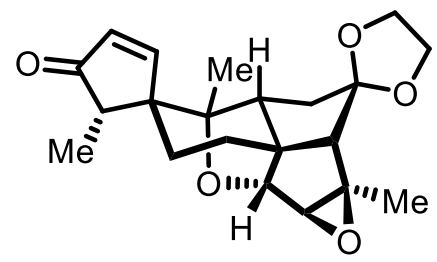

30

${ }^{1} \mathrm{H}$ NMR spectrum $\left(400 \mathrm{MHz}, \mathrm{CDCl}_{3}\right)$

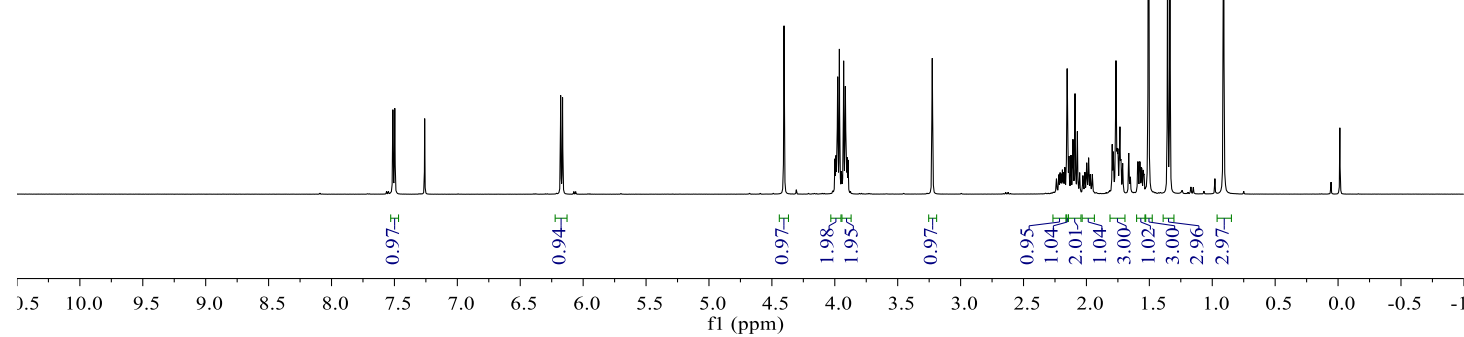

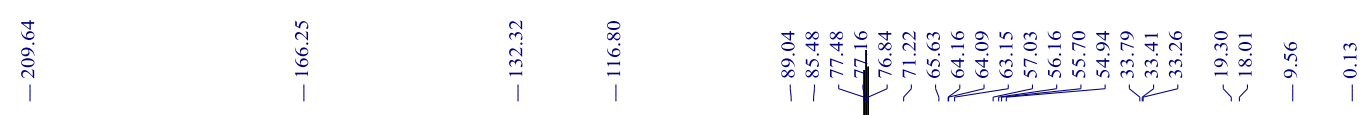

${ }^{13} \mathrm{C}$ NMR spectrum $\left(100 \mathrm{MHz}, \mathrm{CDCl}_{3}\right)$

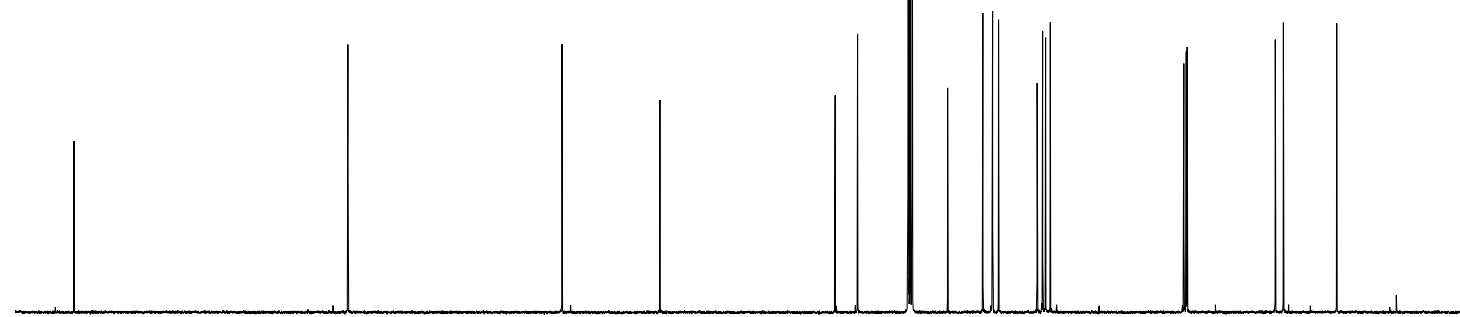

$\begin{array}{lllllllllllllllllllllll}210 & 200 & 190 & 180 & 170 & 160 & 150 & 140 & 130 & 120 & 110 & 100 & 90 & 80 & 70 & 60 & 50 & 40 & 30 & 20 & 10 & 0 & -\end{array}$ 


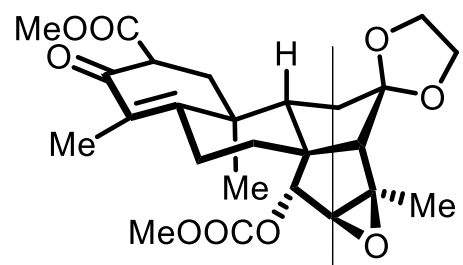

S-7

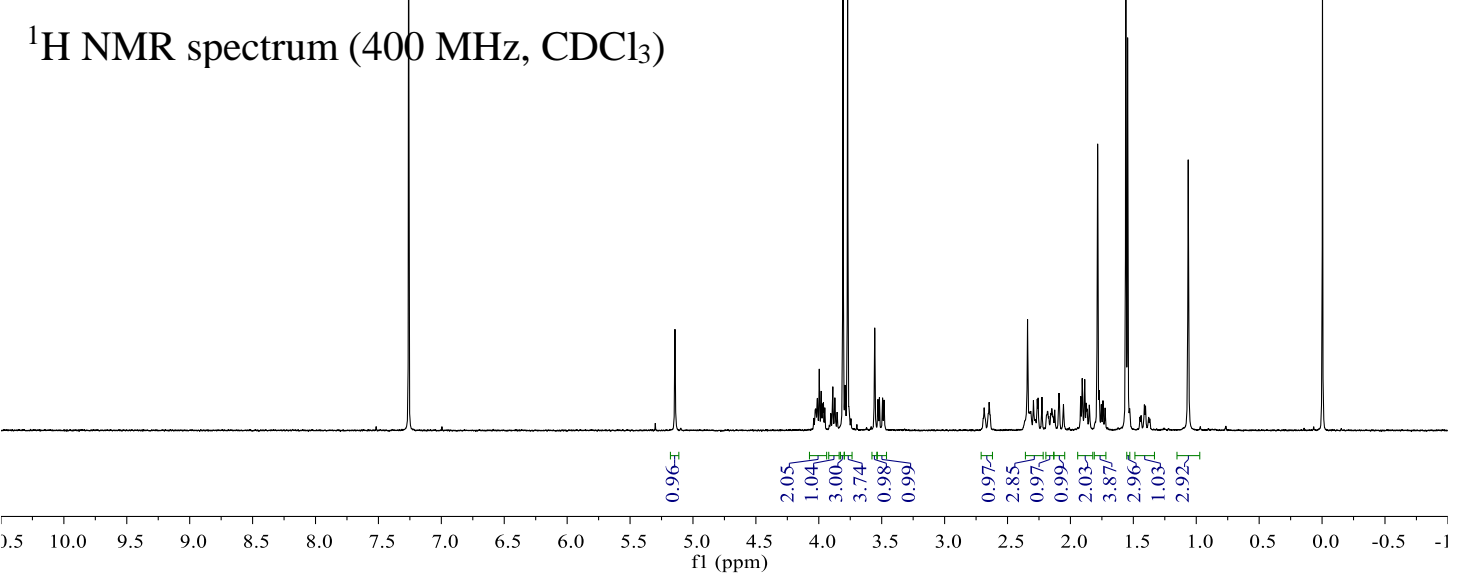

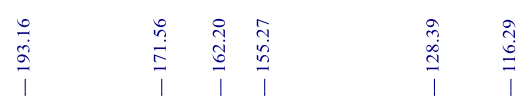

$\underbrace{3}$

${ }^{13} \mathrm{C}$ NMR spectrum $\left(100 \mathrm{MHz}, \mathrm{CDCl}_{3}\right)$

$\begin{array}{rrrrrrrrrrrr}210 & 200 & 190 & 180 & 170 & 160 & 150 & 140 & 130 & 120 & 110 & 100 \\ \text { f1 (ppm) }\end{array}$ 


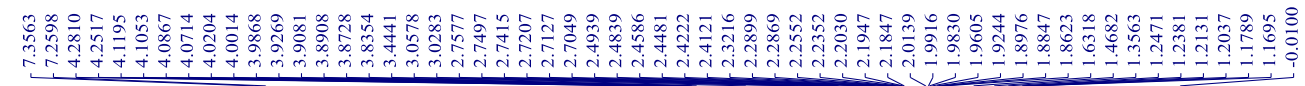

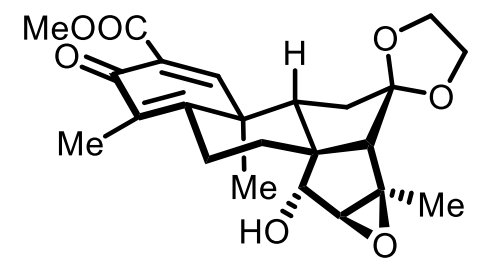

28

${ }^{1} \mathrm{H}$ NMR spectrum $\left(400 \mathrm{MHz}, \mathrm{CDCl}_{3}\right)$

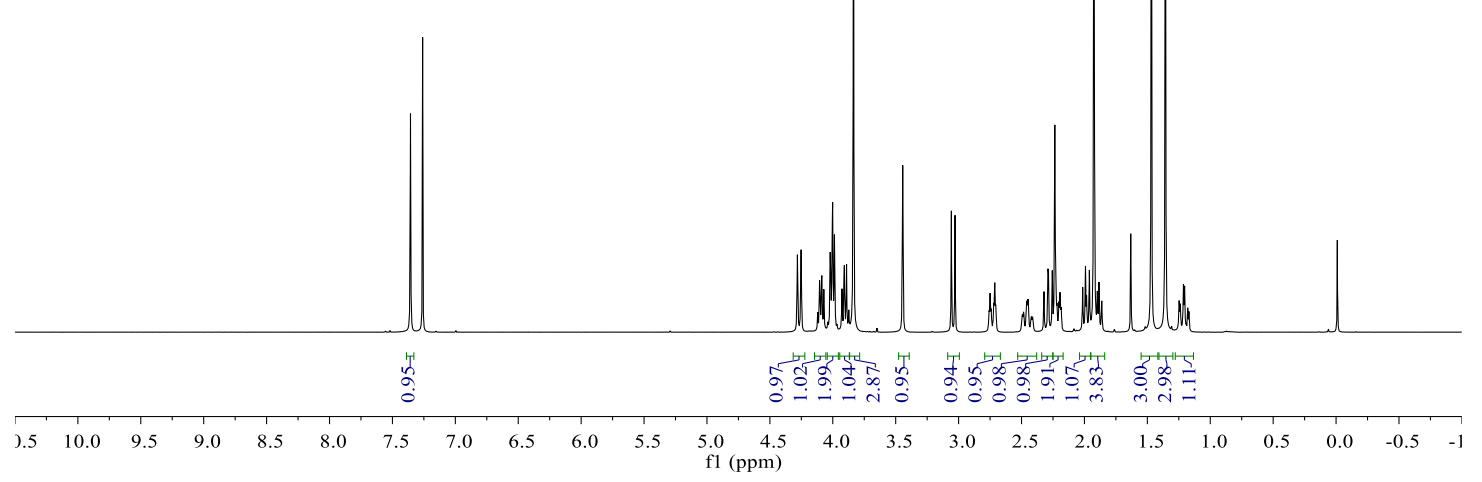

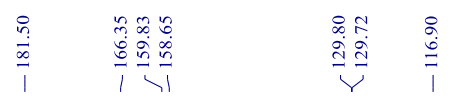

${ }^{13} \mathrm{C}$ NMR spectrum $\left(100 \mathrm{MHz}, \mathrm{CDCl}_{3}\right)$

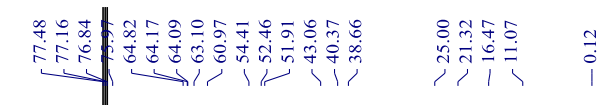

${ }^{3} \mathrm{C} \mathrm{NMR}$ spectrum $\left(100 \mathrm{MH} z, \mathrm{CDCl}_{3}\right)$

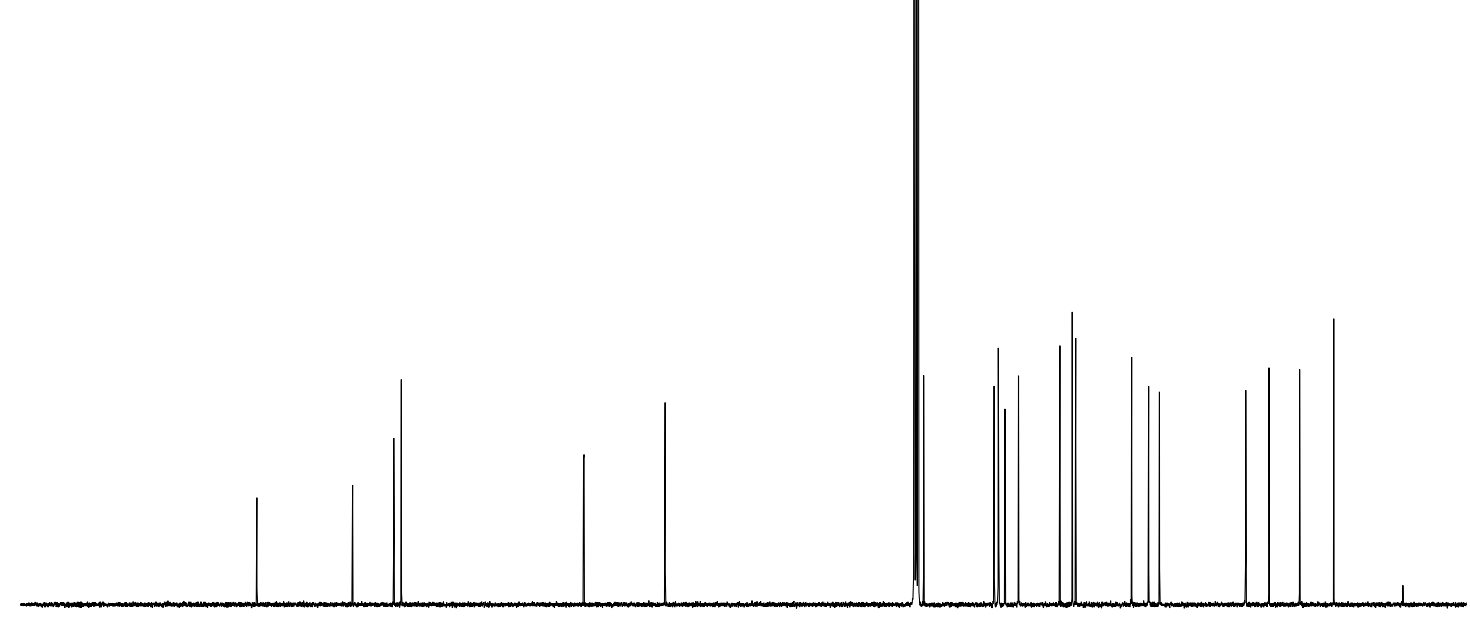

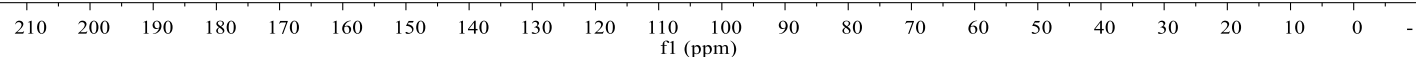



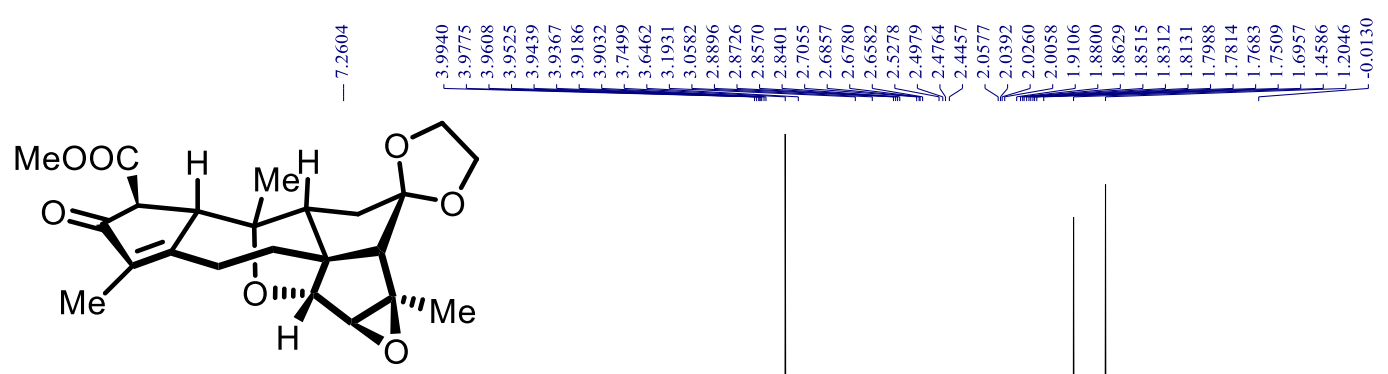

32

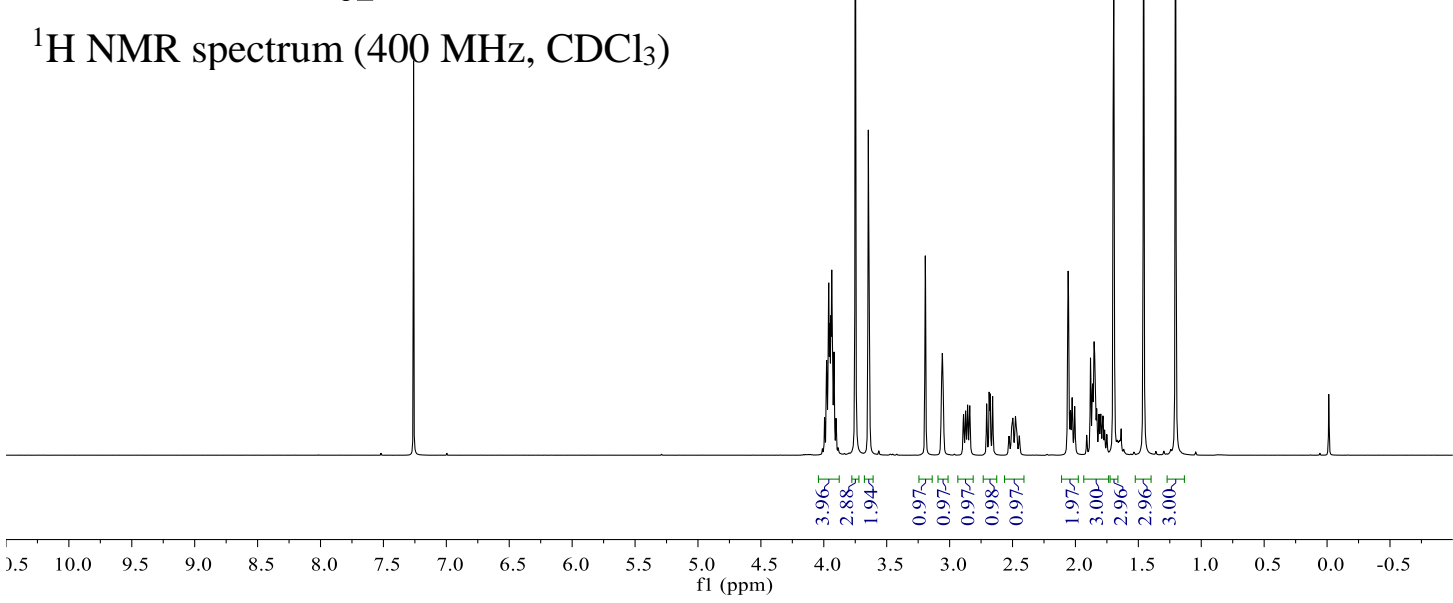

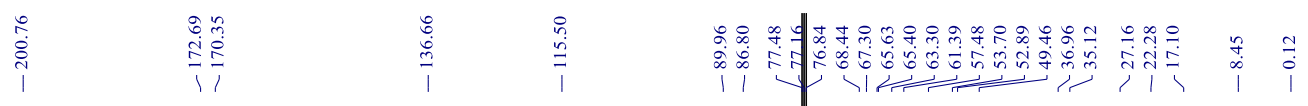

${ }^{13} \mathrm{C}$ NMR spectrum $\left(100 \mathrm{MHz}, \mathrm{CDCl}_{3}\right)$

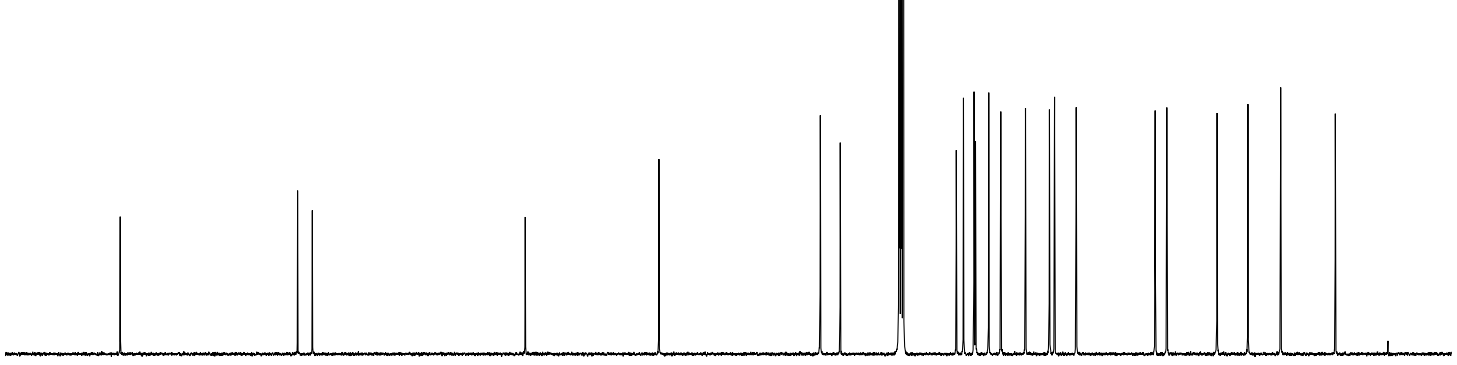

$\begin{array}{lllllllllllllllllllllllllllll}210 & 200 & 190 & 180 & 170 & 160 & 150 & 140 & 130 & 120 & 110 & 100 & 90 & 80 & 70 & 60 & 50 & 40 & 30 & 20 & 10 & 0 & -\end{array}$ 


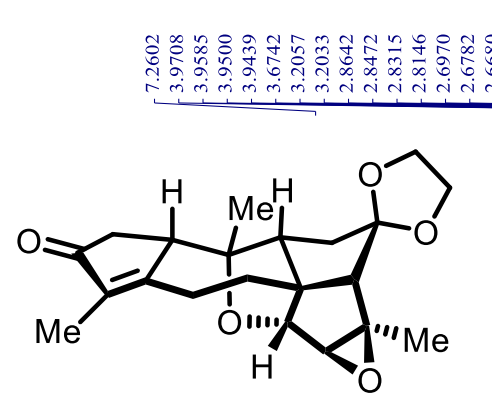

33

${ }^{1} \mathrm{H}$ NMR spectrum $\left(400 \mathrm{MHz}, \mathrm{CDCl}_{3}\right)$

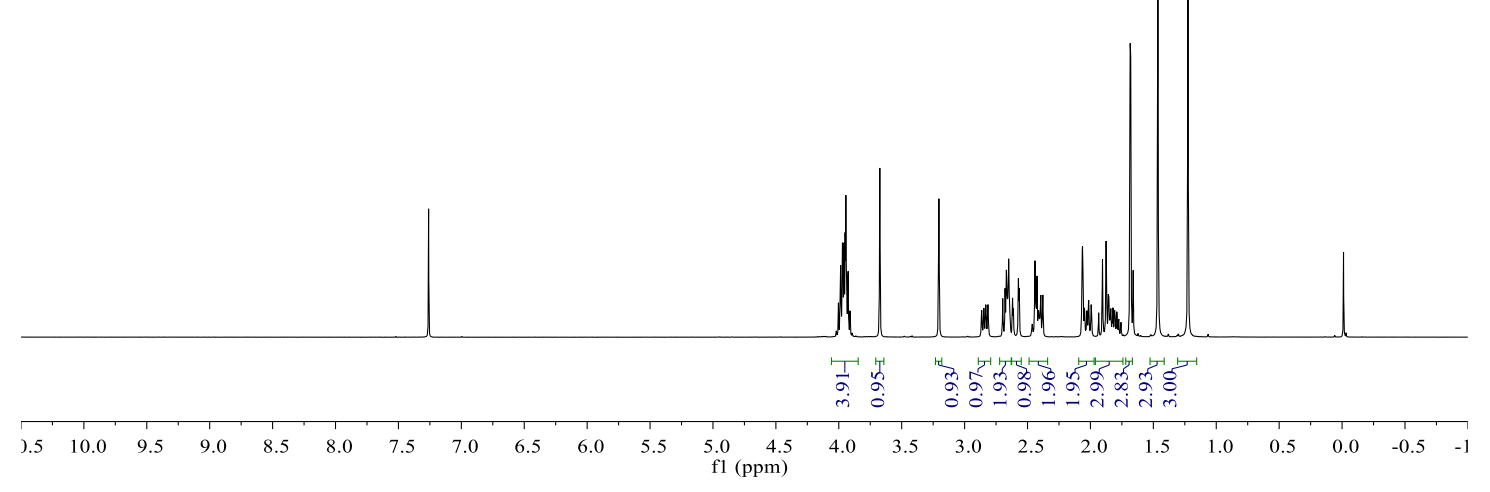

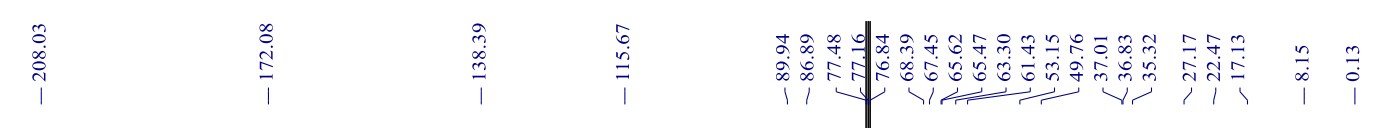

${ }^{13} \mathrm{C}$ NMR spectrum $\left(100 \mathrm{MHz}, \mathrm{CDCl}_{3}\right)$

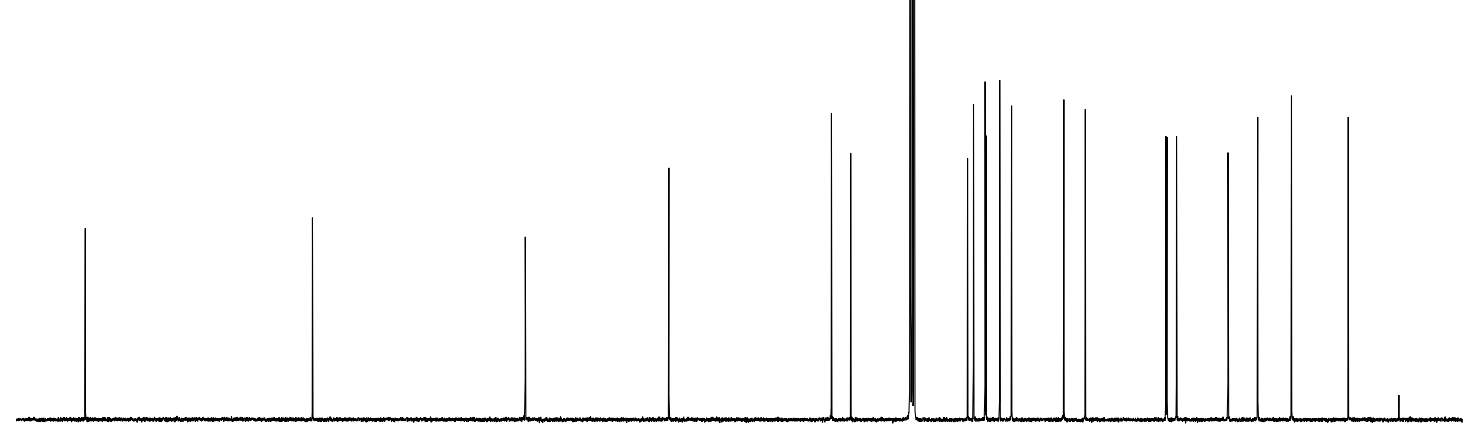

$\begin{array}{lllllllllllllllllllllll}210 & 200 & 190 & 180 & 170 & 160 & 150 & 140 & 130 & 120 & \begin{array}{c}110 \\ \mathrm{f}(\mathrm{ppm})\end{array} & 90 & 80 & 70 & 60 & 50 & 40 & 30 & 20 & 10 & 0 & -\end{array}$ 

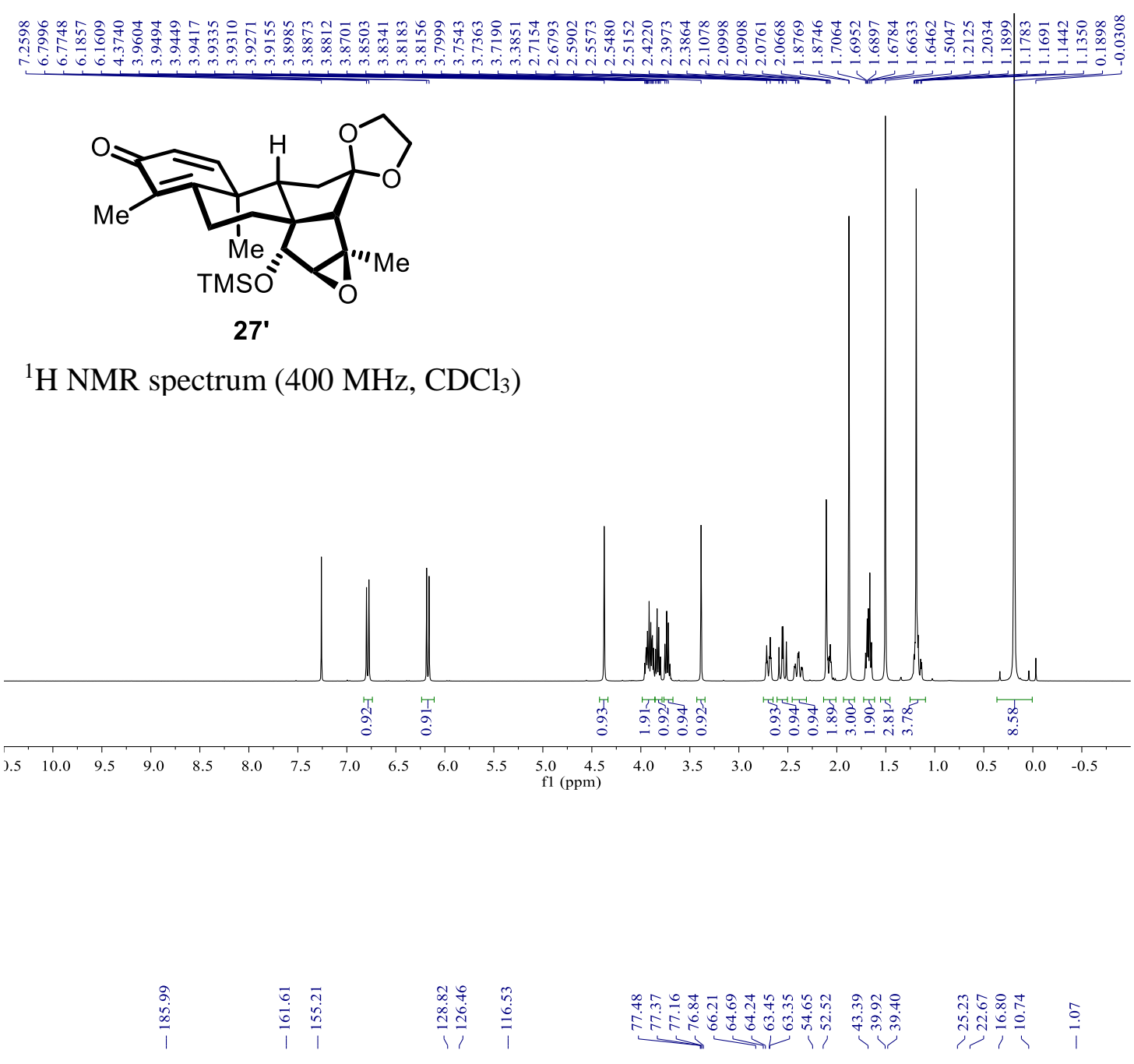

${ }^{13} \mathrm{C}$ NMR spectrum $\left(100 \mathrm{MHz}, \mathrm{CDCl}_{3}\right)$

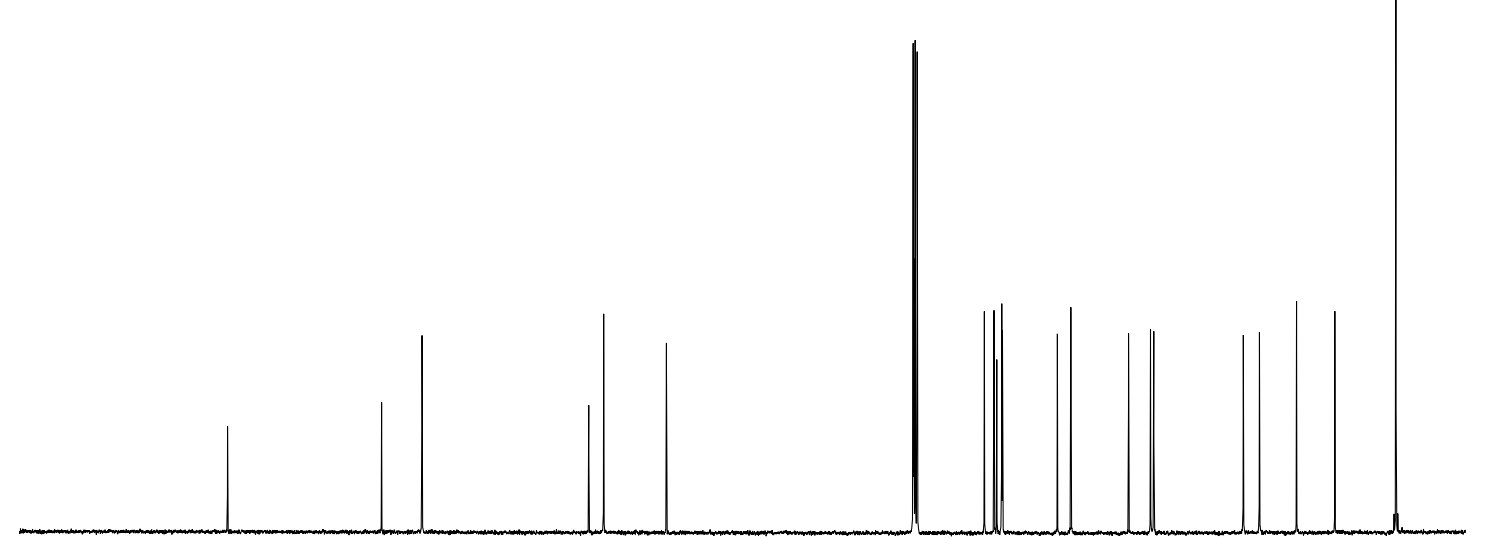

$\begin{array}{lllllllllllllllllllllll}210 & 200 & 190 & 180 & 170 & 160 & 150 & 140 & 130 & 120 & \begin{array}{c}110 \\ \mathrm{f}(\mathrm{ppm})\end{array} & 90 & 80 & 70 & 60 & 50 & 40 & 30 & 20 & 10 & 0 & -\end{array}$ 


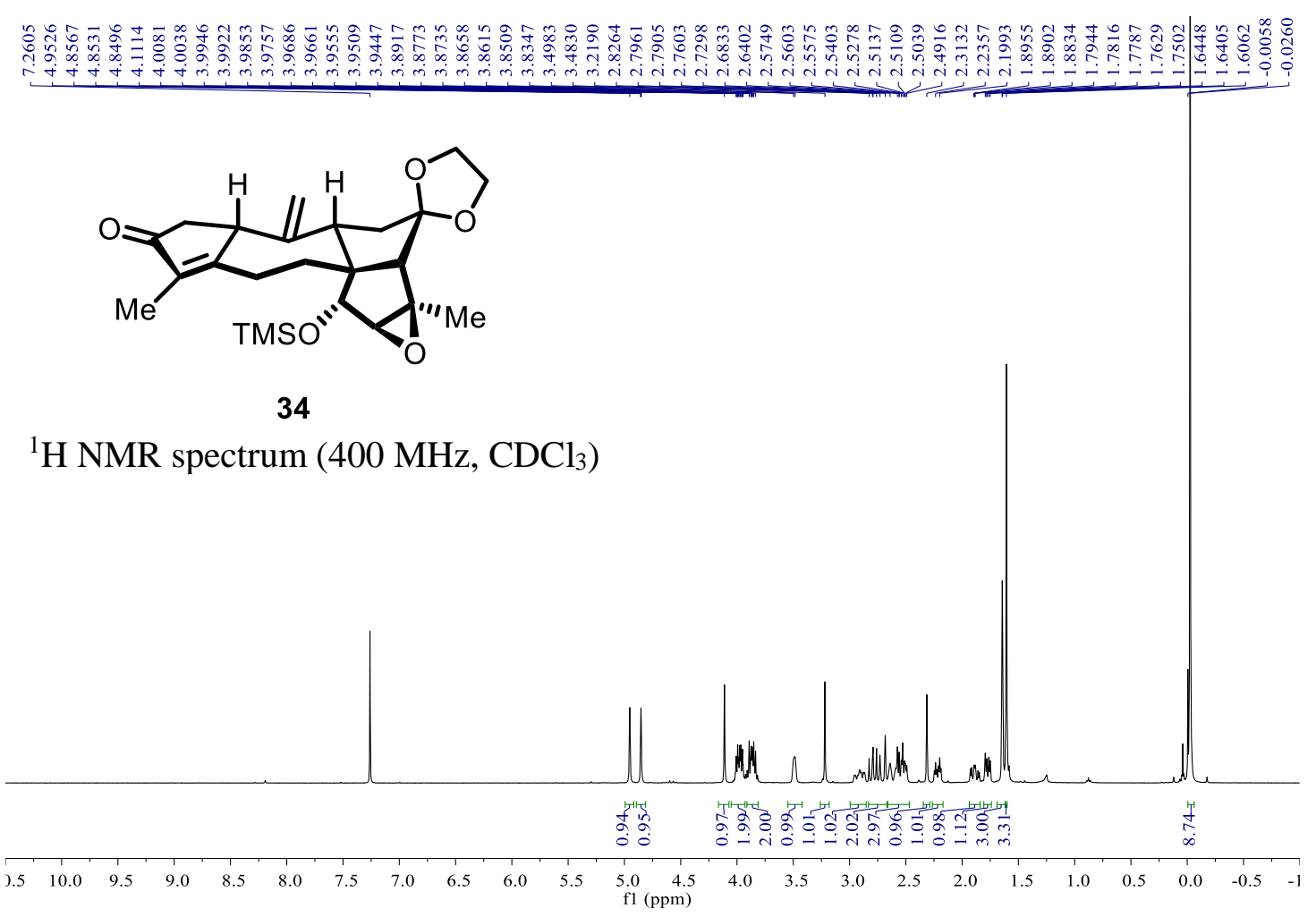

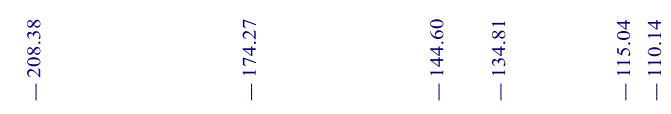

${ }^{13} \mathrm{C}$ NMR spectrum $\left(100 \mathrm{MHz}, \mathrm{CDCl}_{3}\right)$

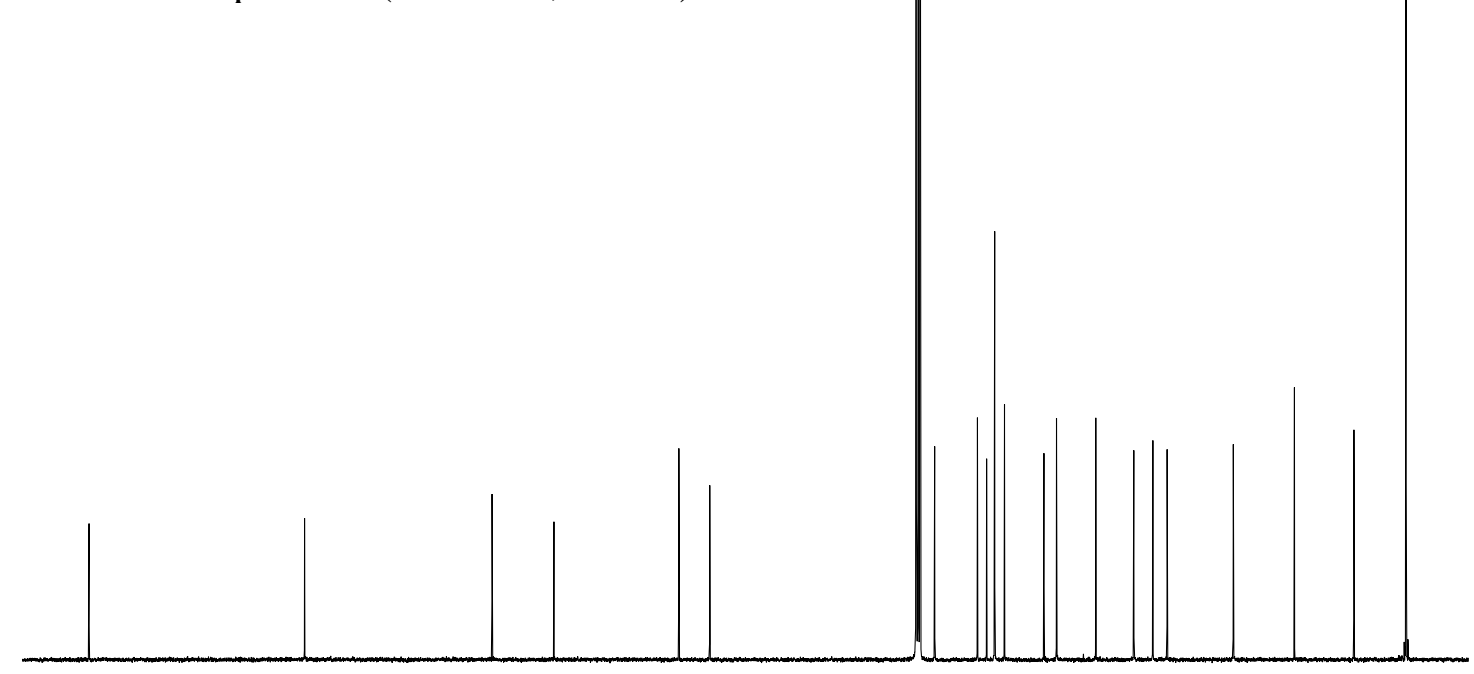

$\begin{array}{lllllllllllllllllllllll}210 & 200 & 190 & 180 & 170 & 160 & 150 & 140 & 130 & 120 & \begin{array}{r}110 \\ \mathrm{f} 1(\mathrm{ppm})\end{array} & 90 & 80 & 70 & 60 & 50 & 40 & 30 & 20 & 10 & 0 & -\end{array}$ 


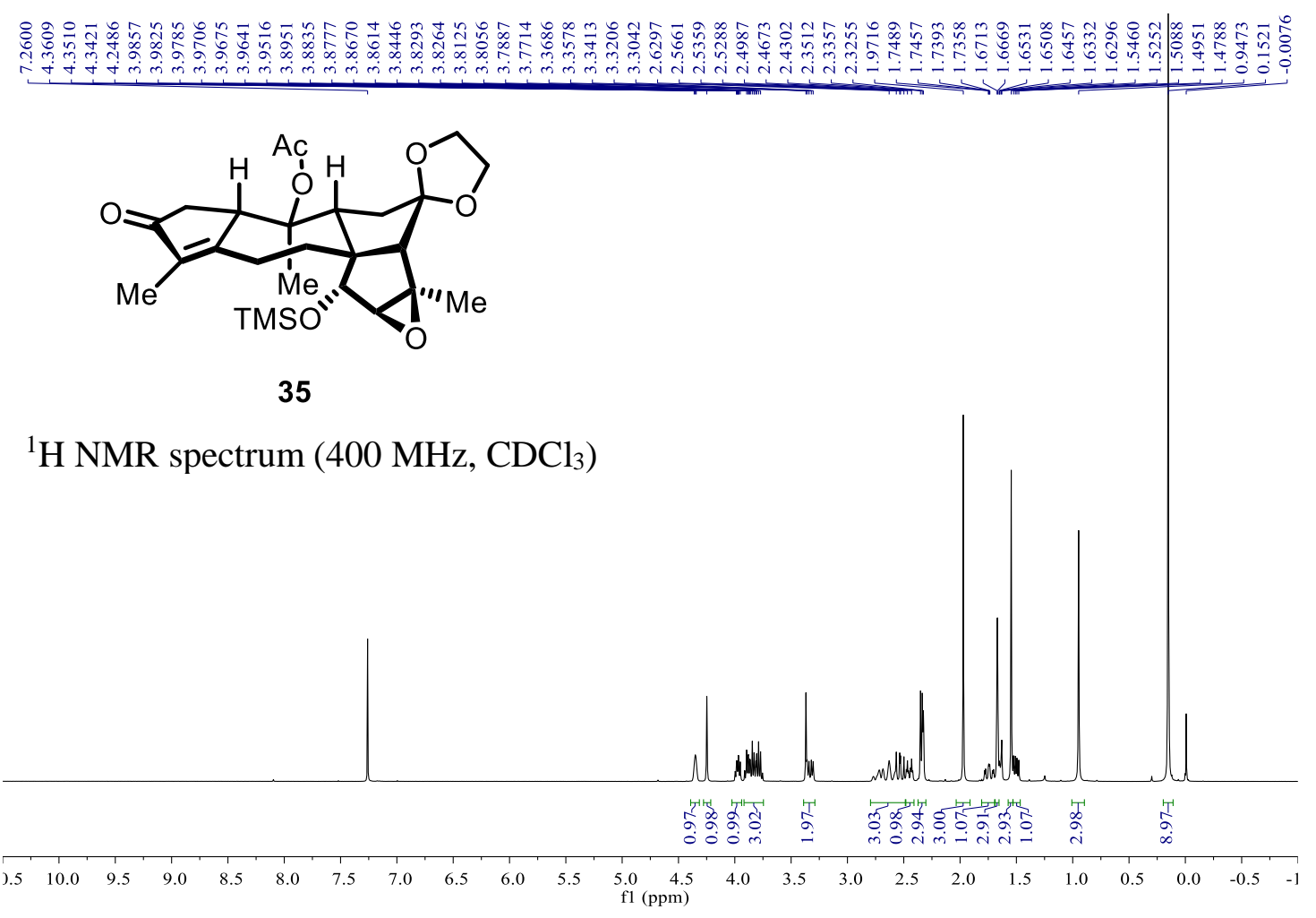

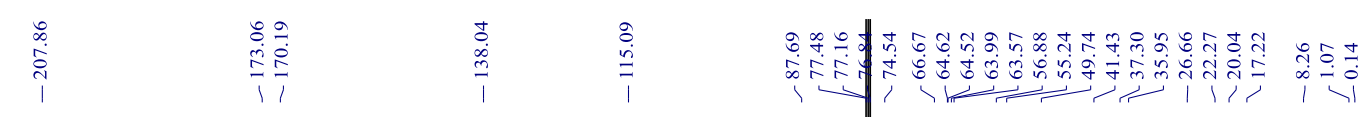

${ }^{13} \mathrm{C}$ NMR spectrum $\left(100 \mathrm{MHz}, \mathrm{CDCl}_{3}\right)$

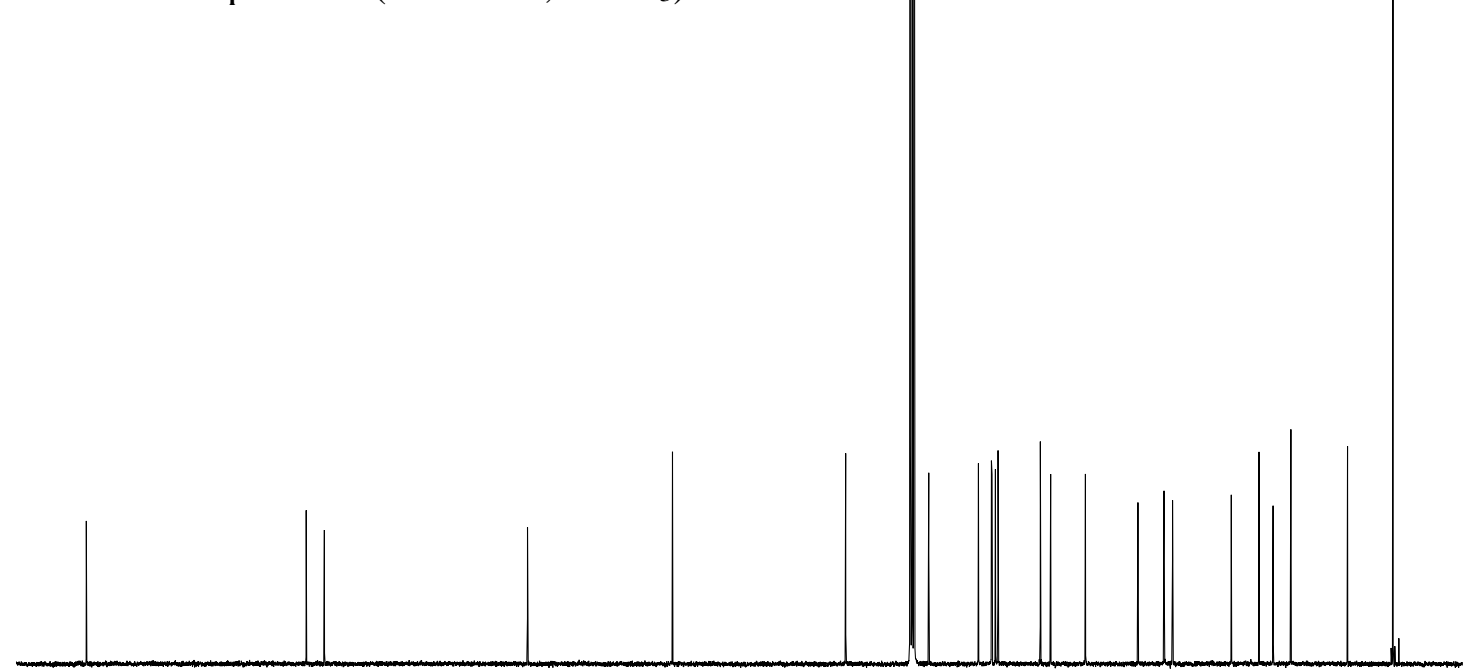

$\begin{array}{lllllllllllllllllllllll}210 & 200 & 190 & 180 & 170 & 160 & 150 & 140 & 130 & 120 & \begin{array}{c}110 \\ \mathrm{f} 1(\mathrm{ppm})\end{array} & 90 & 80 & 70 & 60 & 50 & 40 & 30 & 20 & 10 & 0 & -\end{array}$ 


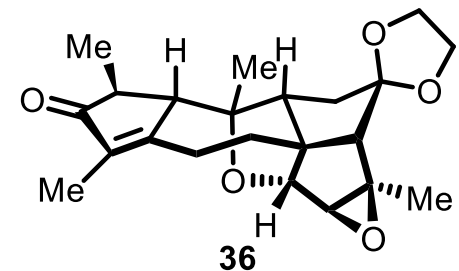

${ }^{1} \mathrm{H} \mathrm{NMR}$ spectrum $\left(400 \mathrm{MHz}, \mathrm{CDCl}_{3}\right)$

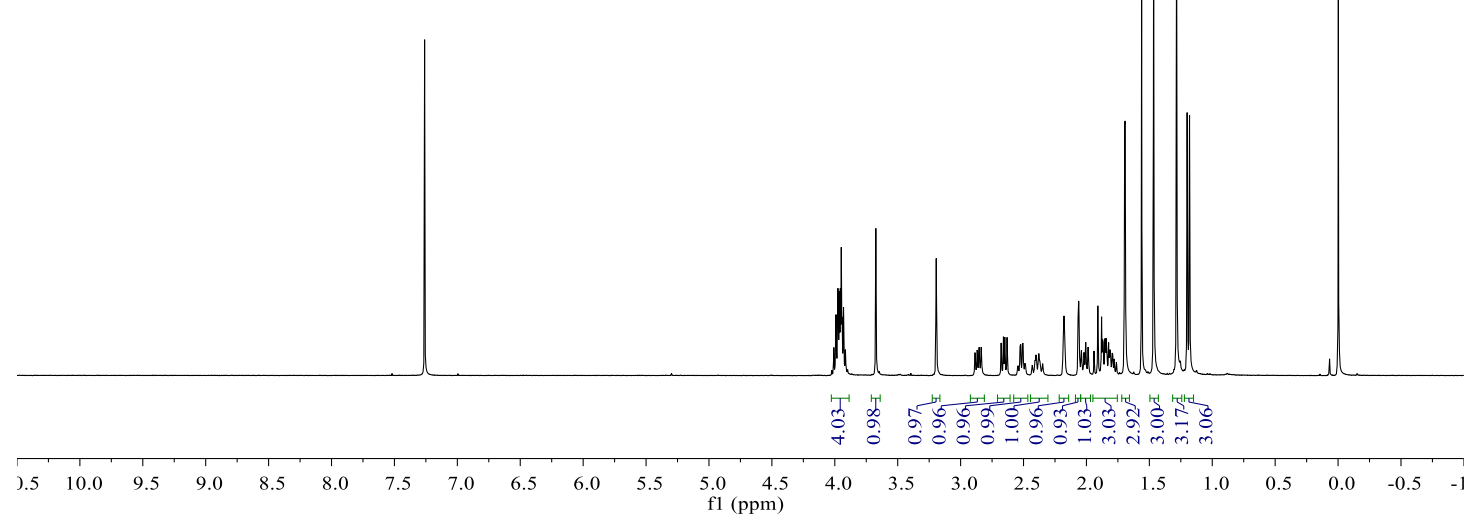

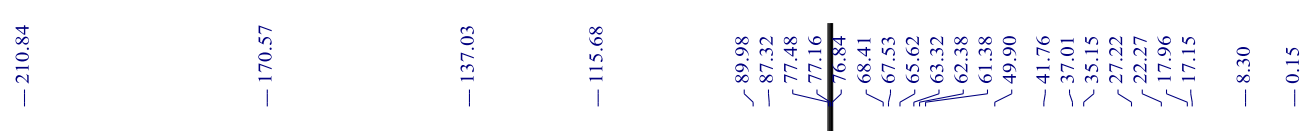

${ }^{13} \mathrm{C}$ NMR spectrum $\left(100 \mathrm{MHz}, \mathrm{CDCl}_{3}\right)$

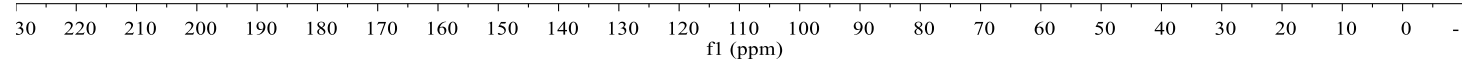




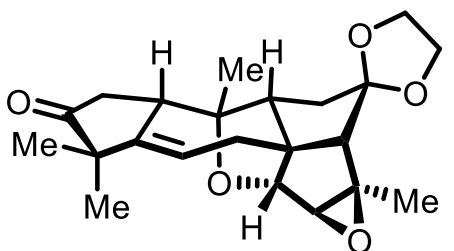

37

${ }^{1} \mathrm{H}$ NMR spectrum $\left(400 \mathrm{MHz}, \mathrm{CDCl}_{3}\right)$

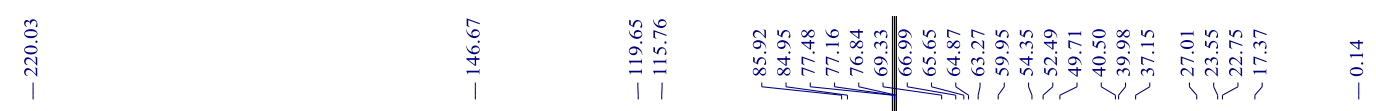

${ }^{13} \mathrm{C}$ NMR spectrum $\left(100 \mathrm{MHz}, \mathrm{CDCl}_{3}\right)$

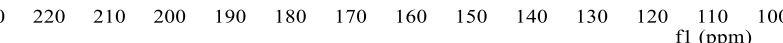




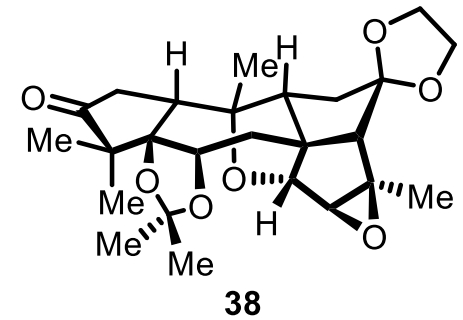

${ }^{1} \mathrm{H}$ NMR spectrum $\left(400 \mathrm{MHz}, \mathrm{CDCl}_{3}\right)$

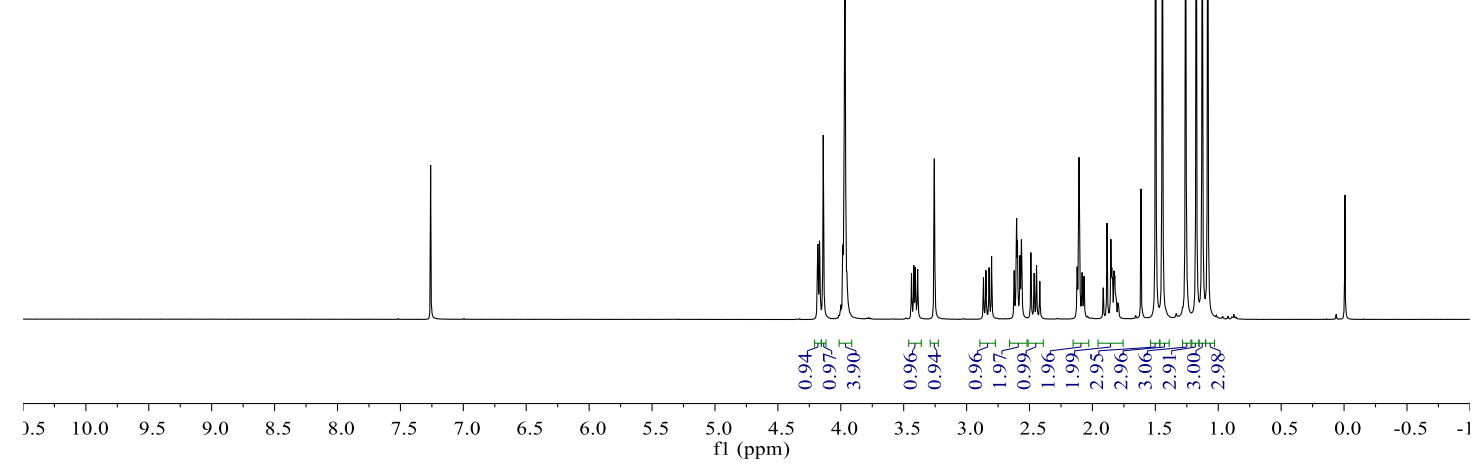

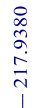

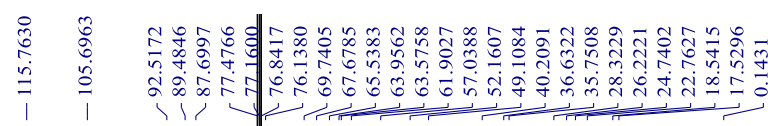

${ }^{13} \mathrm{C}$ NMR spectrum $\left(100 \mathrm{MHz}, \mathrm{CDCl}_{3}\right)$

$\begin{array}{lllllllllllllllllllllllllllllllll}30 & 220 & 210 & 200 & 190 & 180 & 170 & 160 & 150 & 140 & 130 & 120 & 110 & 100 & 90 & 80 & 70 & 60 & 50 & 40 & 30 & 20 & 10 & 0\end{array}$ 


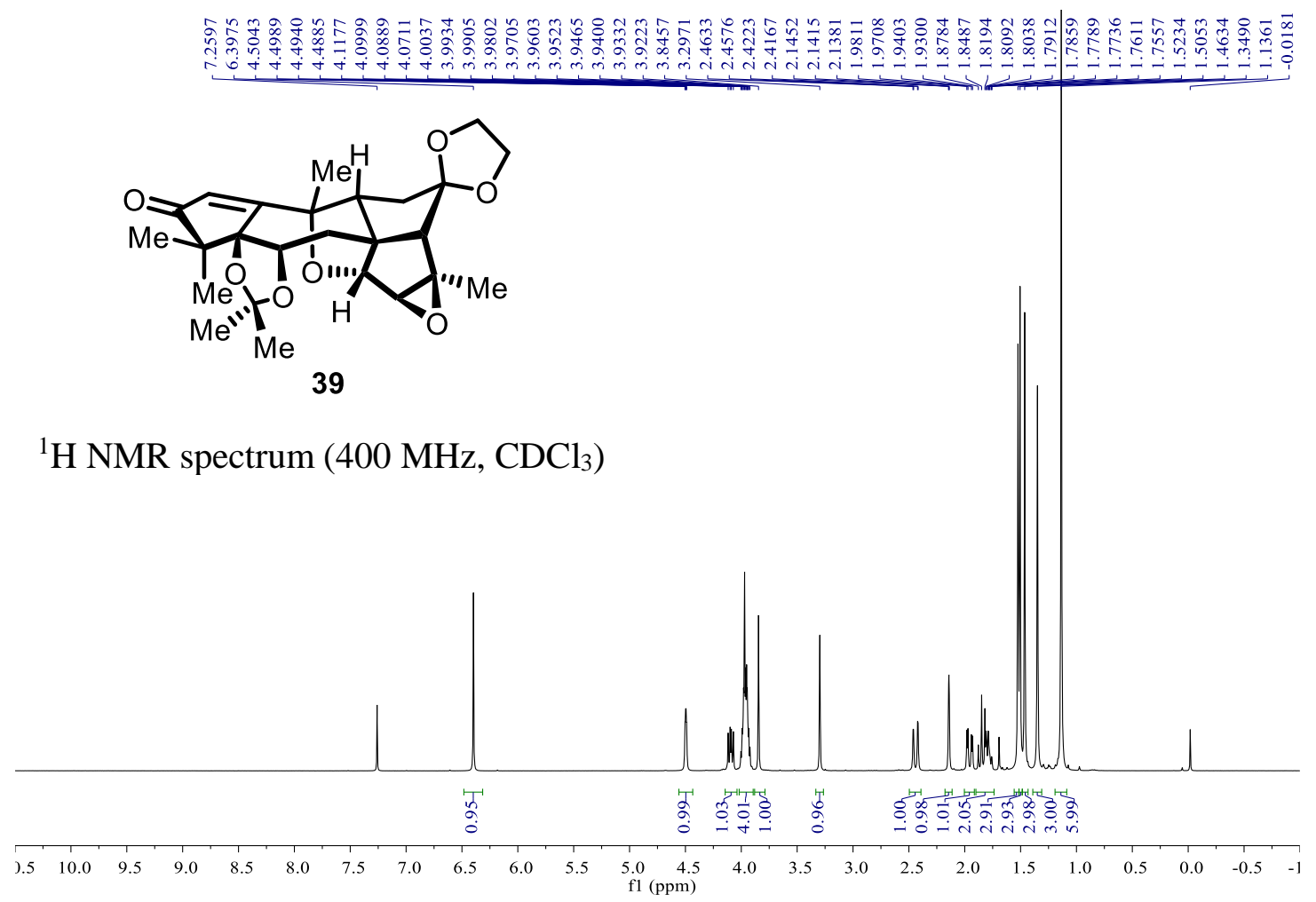

${ }^{1} \mathrm{H}$ NMR spectrum $\left(400 \mathrm{MHz}, \mathrm{CDCl}_{3}\right)$

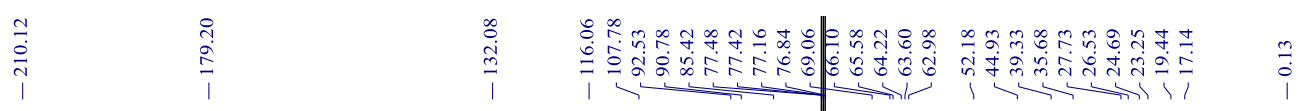

${ }^{13} \mathrm{C}$ NMR spectrum $\left(100 \mathrm{MHz}, \mathrm{CDCl}_{3}\right)$

$\begin{array}{lllllllllllllllllllllllllllllll}10 & 220 & 210 & 200 & 190 & 180 & 170 & 160 & 150 & 140 & 130 & 120 & 110 & 100 & 90 & 80 & 70 & 60 & 50 & 40 & 30 & 20 & 10 & 0 & -\end{array}$ 


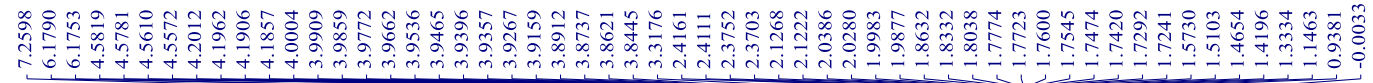

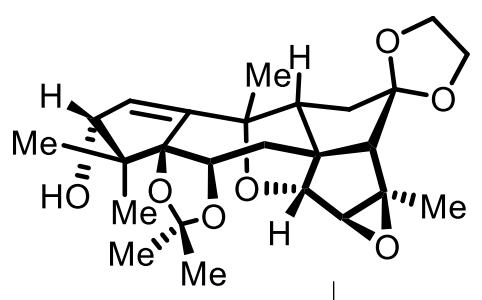

S-9

${ }^{1} \mathrm{H}$ NMR spectrum $\left(400 \mathrm{MHz}, \mathrm{CDCl}_{3}\right)$

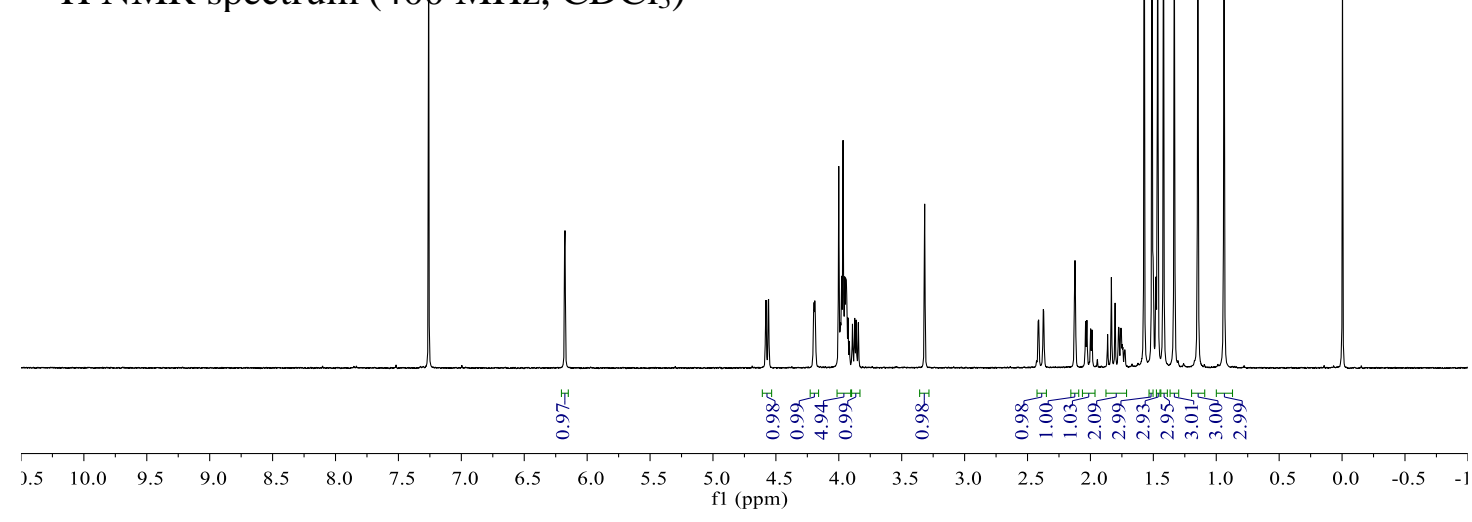

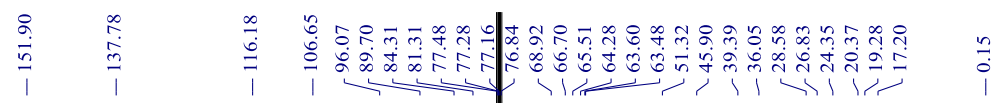

${ }^{13} \mathrm{C}$ NMR spectrum $\left(100 \mathrm{MHz}, \mathrm{CDCl}_{3}\right)$

$\begin{array}{llllllllllll}210 & 200 & 190 & 180 & 170 & 160 & 150 & 140 & 130 & 120 & 110 \begin{array}{l}100 \\ \text { f1 (ppm) }\end{array}\end{array}$ 

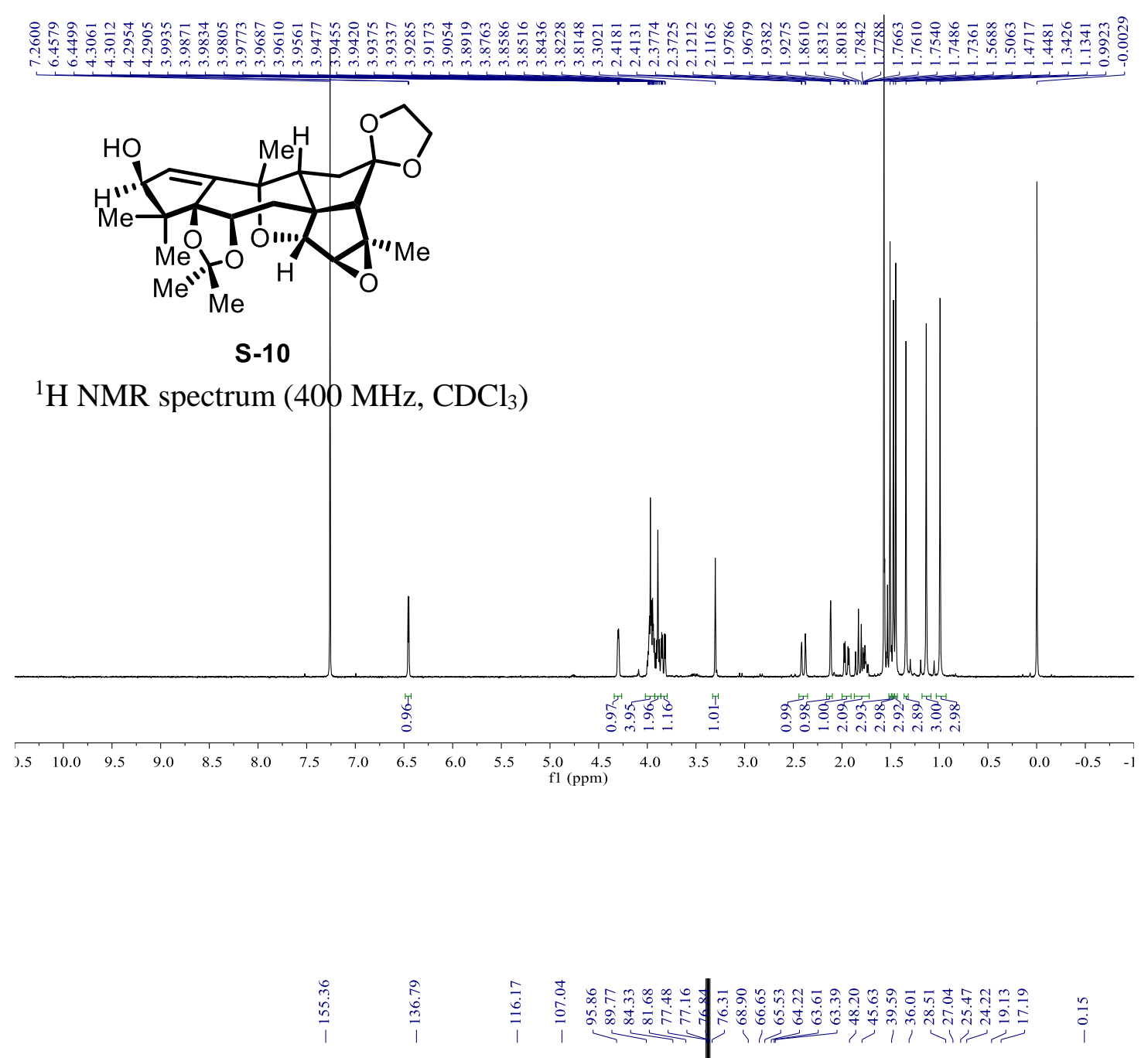

${ }^{13} \mathrm{C}$ NMR spectrum $\left(100 \mathrm{MHz}, \mathrm{CDCl}_{3}\right)$

$\begin{array}{lllllllllllllllllllllll}210 & 200 & 190 & 180 & 170 & 160 & 150 & 140 & 130 & 120 & 110 & 100 & 90 & 80 & 70 & 60 & 50 & 40 & 30 & 20 & 10 & 0 & -\end{array}$ 


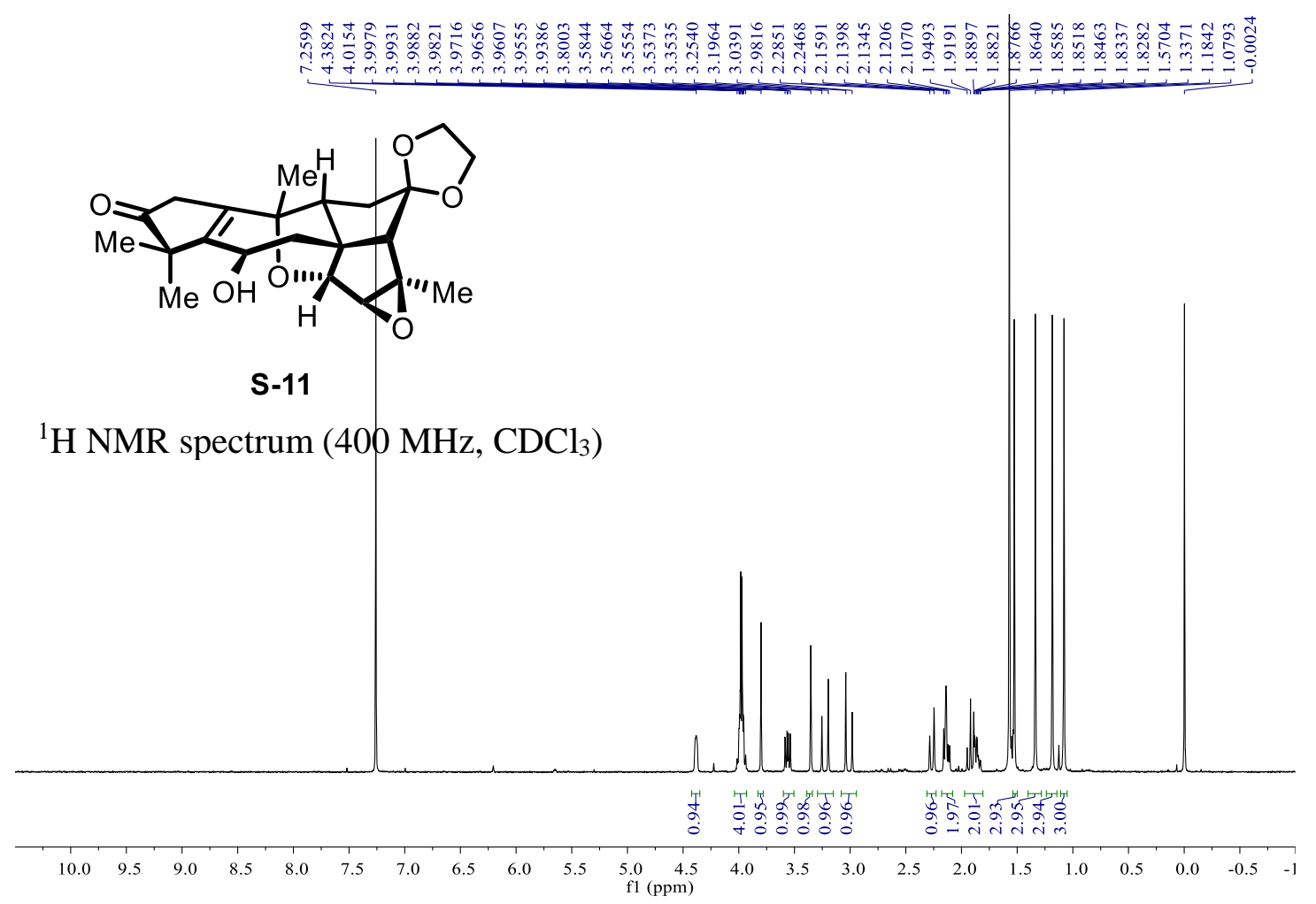

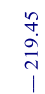

${ }^{13} \mathrm{C}$ NMR spectrum $\left(100 \mathrm{MHz}, \mathrm{CDCl}_{3}\right)$

$\begin{array}{lllllllllllll}1 & 220 & 210 & 200 & 190 & 180 & 170 & 160 & 150 & 140 & 130 & 120 \begin{array}{l}110 \\ \mathrm{f} 1(\mathrm{ppm})\end{array} & 100\end{array}$ 


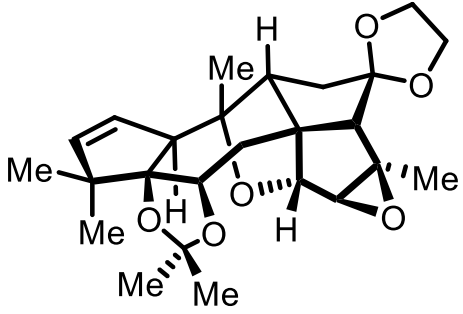

41

${ }^{1} \mathrm{H}$ NMR spectrum (400 MHz, acetone- $\left.d_{6}\right)$

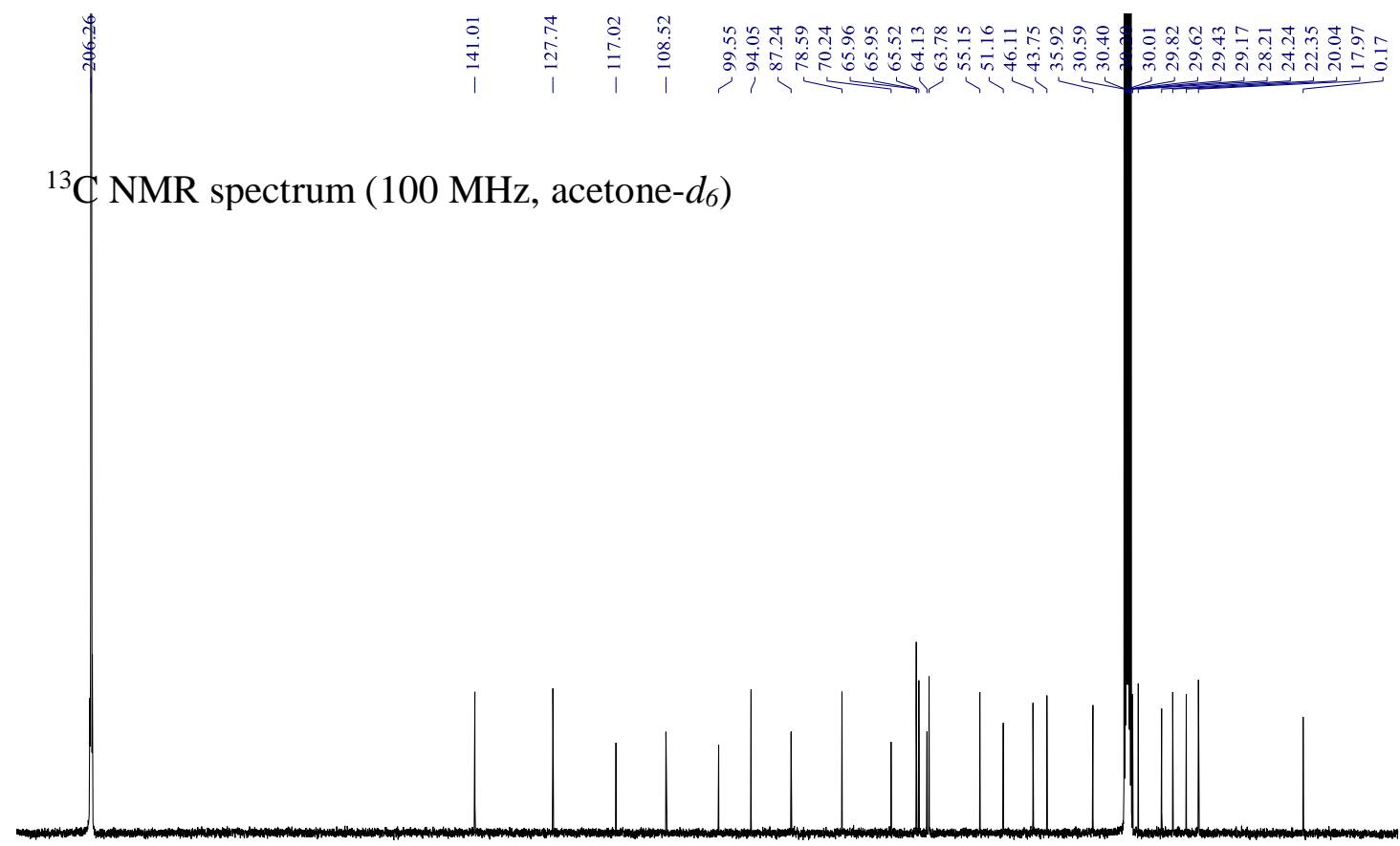

$\begin{array}{lllllllllllllllllllllll}210 & 200 & 190 & 180 & 170 & 160 & 150 & 140 & 130 & 120 & 110 & 100 & 90 & 80 & 70 & 60 & 50 & 40 & 30 & 20 & 10 & 0 & -10\end{array}$ 

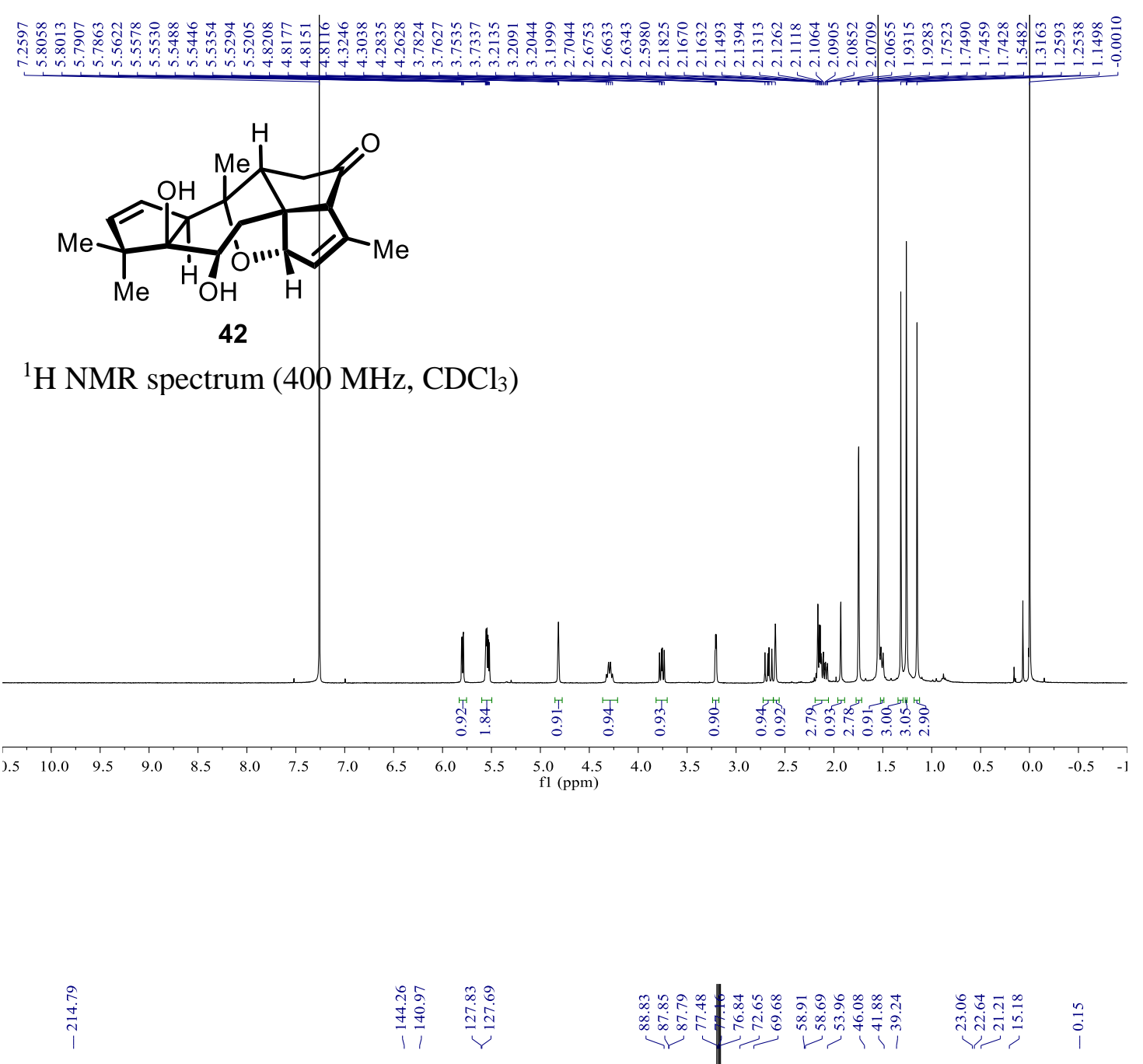

${ }^{13} \mathrm{C}$ NMR spectrum $\left(100 \mathrm{MHz}, \mathrm{CDCl}_{3}\right)$

$\begin{array}{llllllllllllllllllllllllllll}30 & 220 & 210 & 200 & 190 & 180 & 170 & 160 & 150 & 140 & 130 & 120 & \begin{array}{l}110 \\ \mathrm{f} 1(\mathrm{ppm})\end{array} & 100 & 90 & 80 & 70 & 60 & 50 & 40 & 30 & 20 & 10 & 0 & -\end{array}$

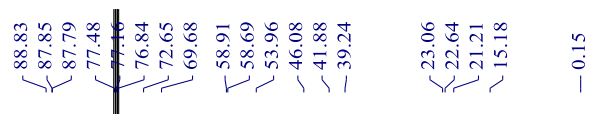

\section{3.}

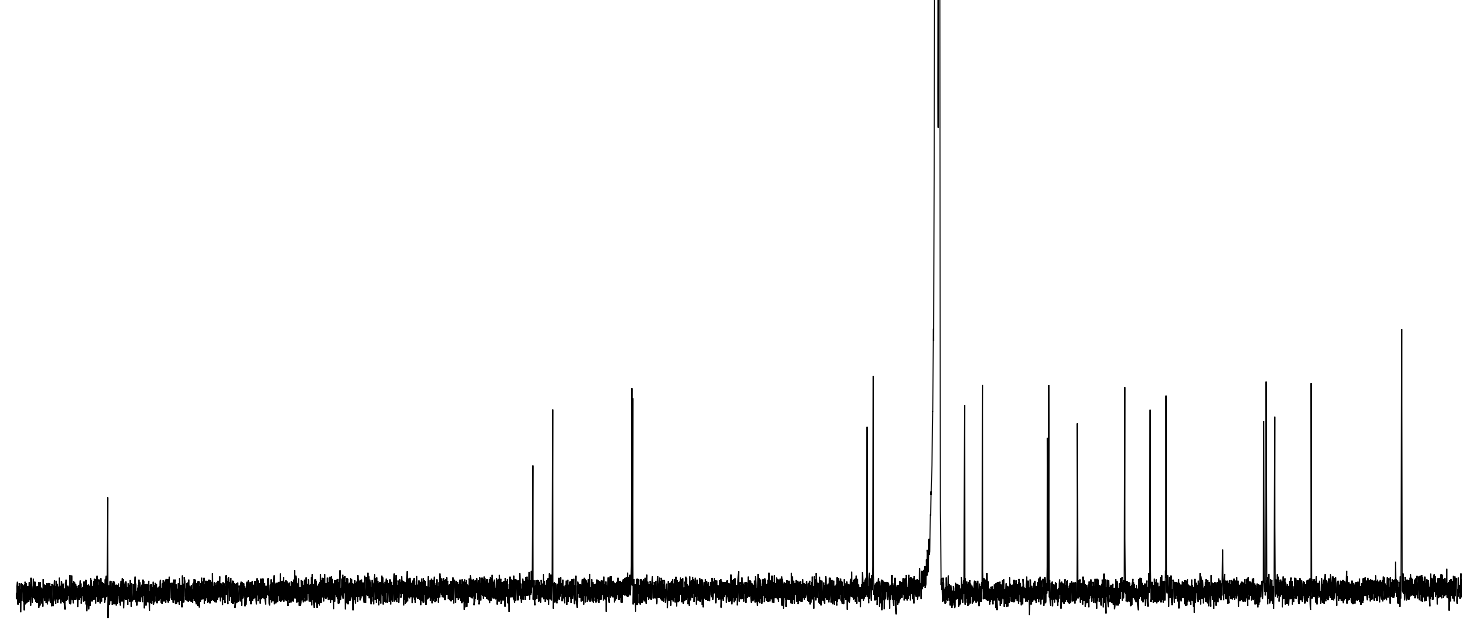




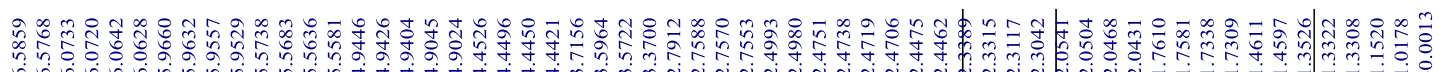

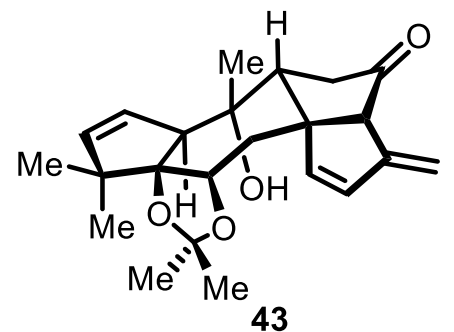

${ }^{1} \mathrm{H}$ NMR spectrum $\left(600 \mathrm{MHz}\right.$, acetone- $\left.d_{6}\right)$
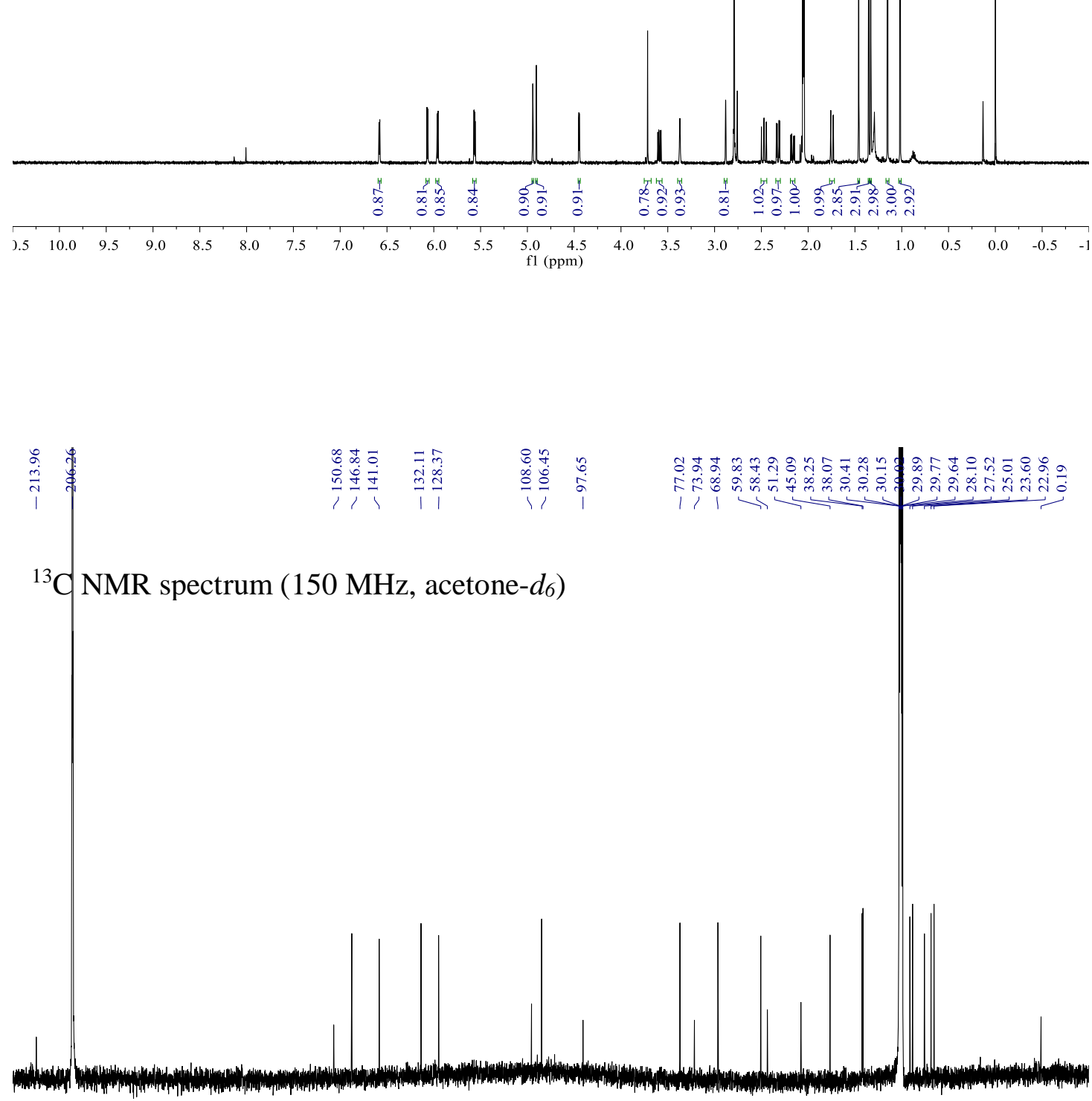

$\begin{array}{lllllllllllllllllllllll}210 & 200 & 190 & 180 & 170 & 160 & 150 & 140 & 130 & 120 & 110 & 100 & 90 & 80 & 70 & 60 & 50 & 40 & 30 & 20 & 10 & 0 & -\end{array}$ 


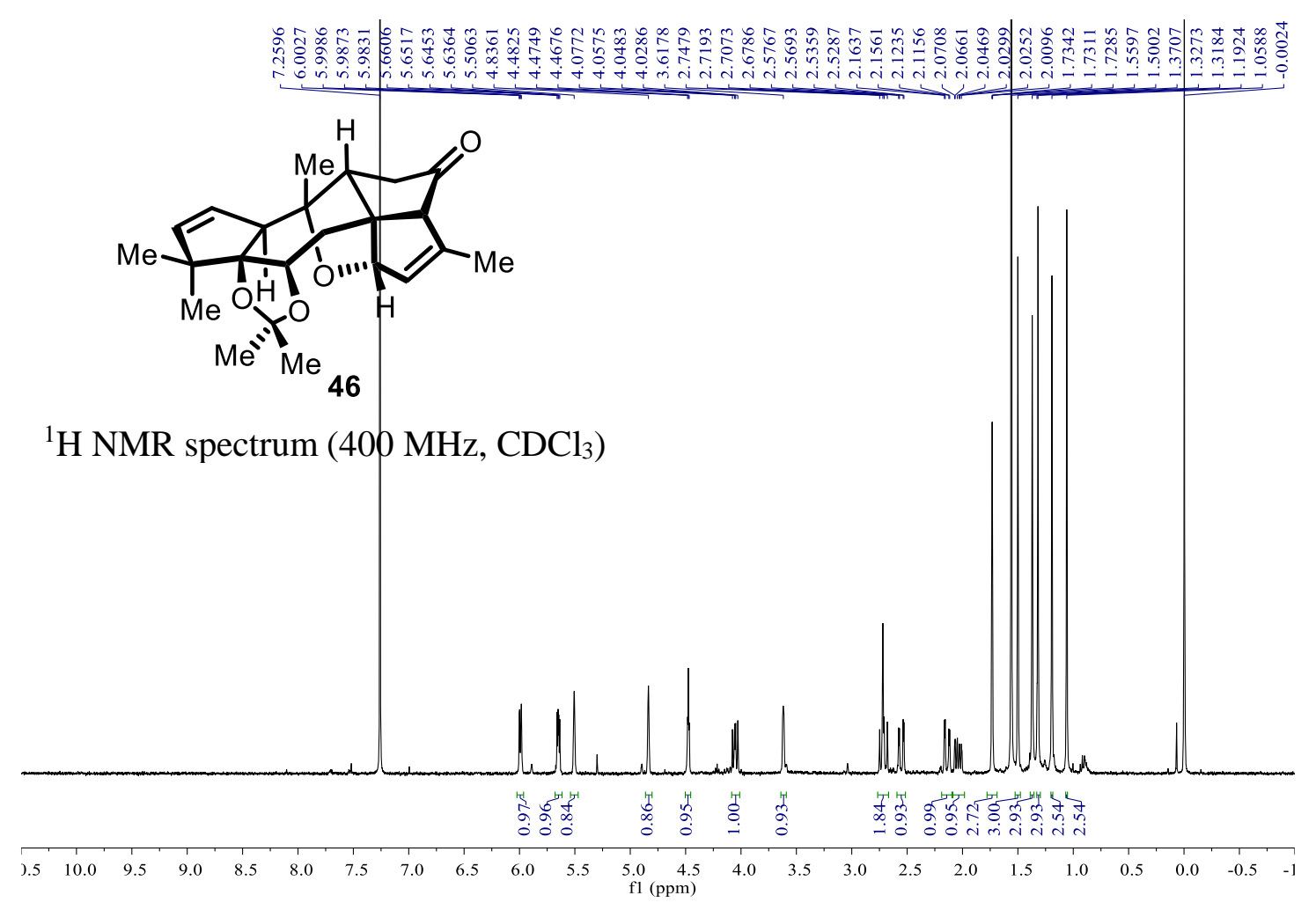

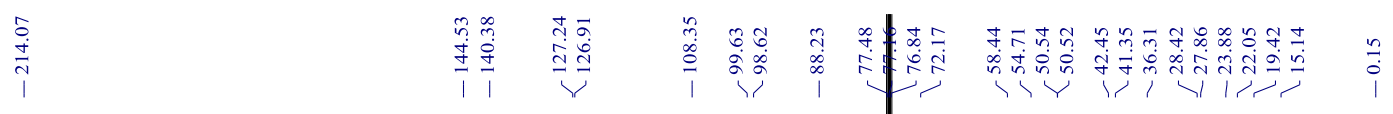

${ }^{13} \mathrm{C}$ NMR spectrum $\left(100 \mathrm{MHz}, \mathrm{CDCl}_{3}\right)$

$\begin{array}{lllllllllllllllllllllll}210 & 200 & 190 & 180 & 170 & 160 & 150 & 140 & 130 & 120 & 110 & 100 & 90 & 80 & 70 & 60 & 50 & 40 & 30 & 20 & 10 & 0 & -\end{array}$ 


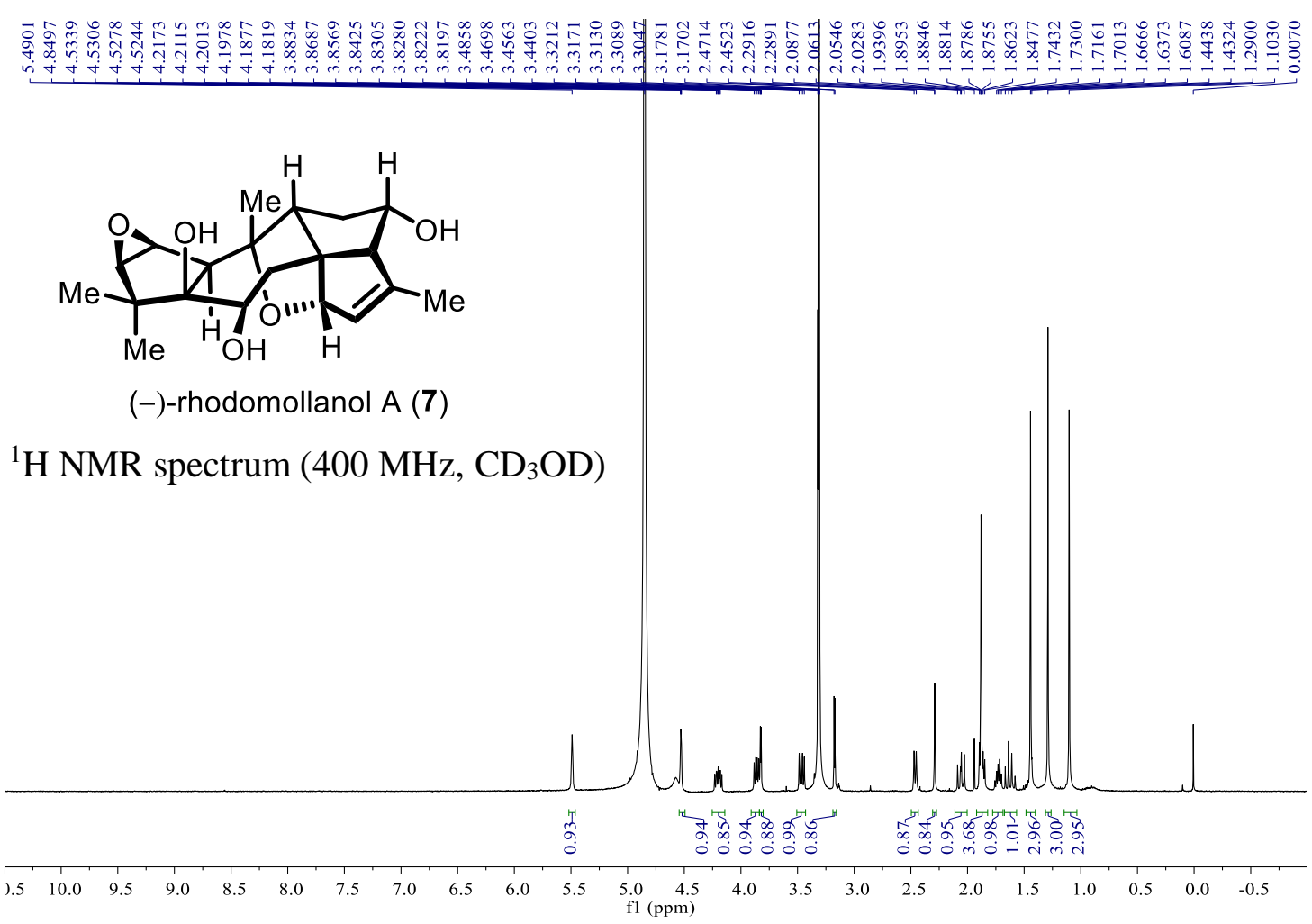

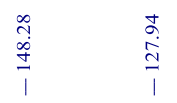

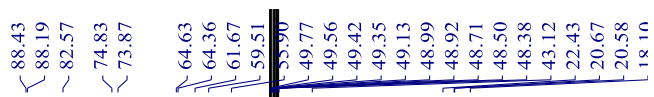

${ }^{13} \mathrm{C}$ NMR spectrum $\left(100 \mathrm{MHz}, \mathrm{CD}_{3} \mathrm{OD}\right)$

$\begin{array}{lllllllllllllllllllllll}210 & 200 & 190 & 180 & 170 & 160 & 150 & 140 & 130 & 120 & 110 & 100 & 90 & 80 & 70 & 60 & 50 & 40 & 30 & 20 & 10 & 0 & -\end{array}$ 

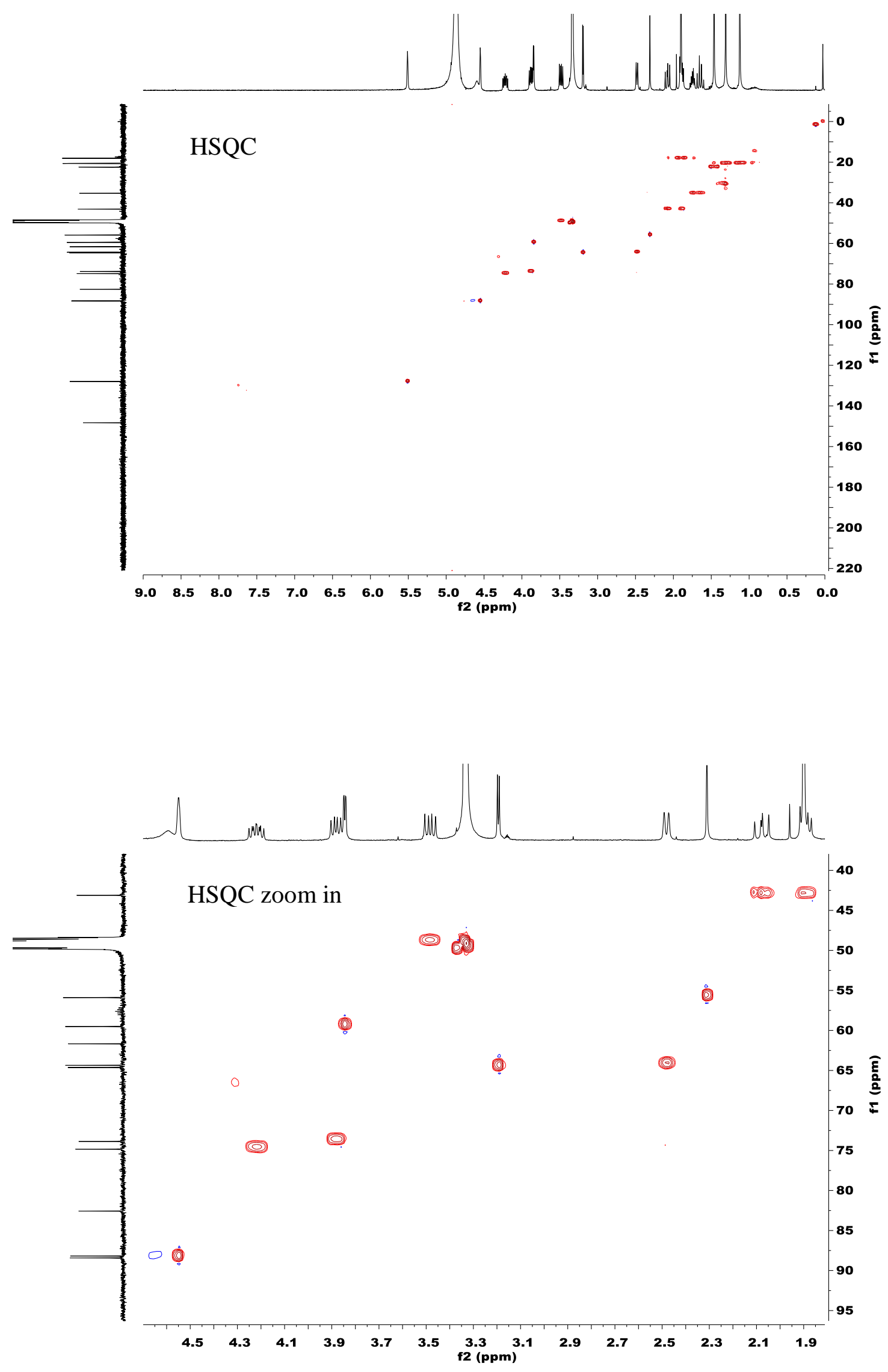
Figure S1. Comparison of ${ }^{1} \mathrm{H}$ NMR Spectra of Synthetic and Natural (-)Rhodomollanol A $\left(\mathrm{CD}_{3} \mathrm{OD}, 25^{\circ} \mathrm{C}\right)$
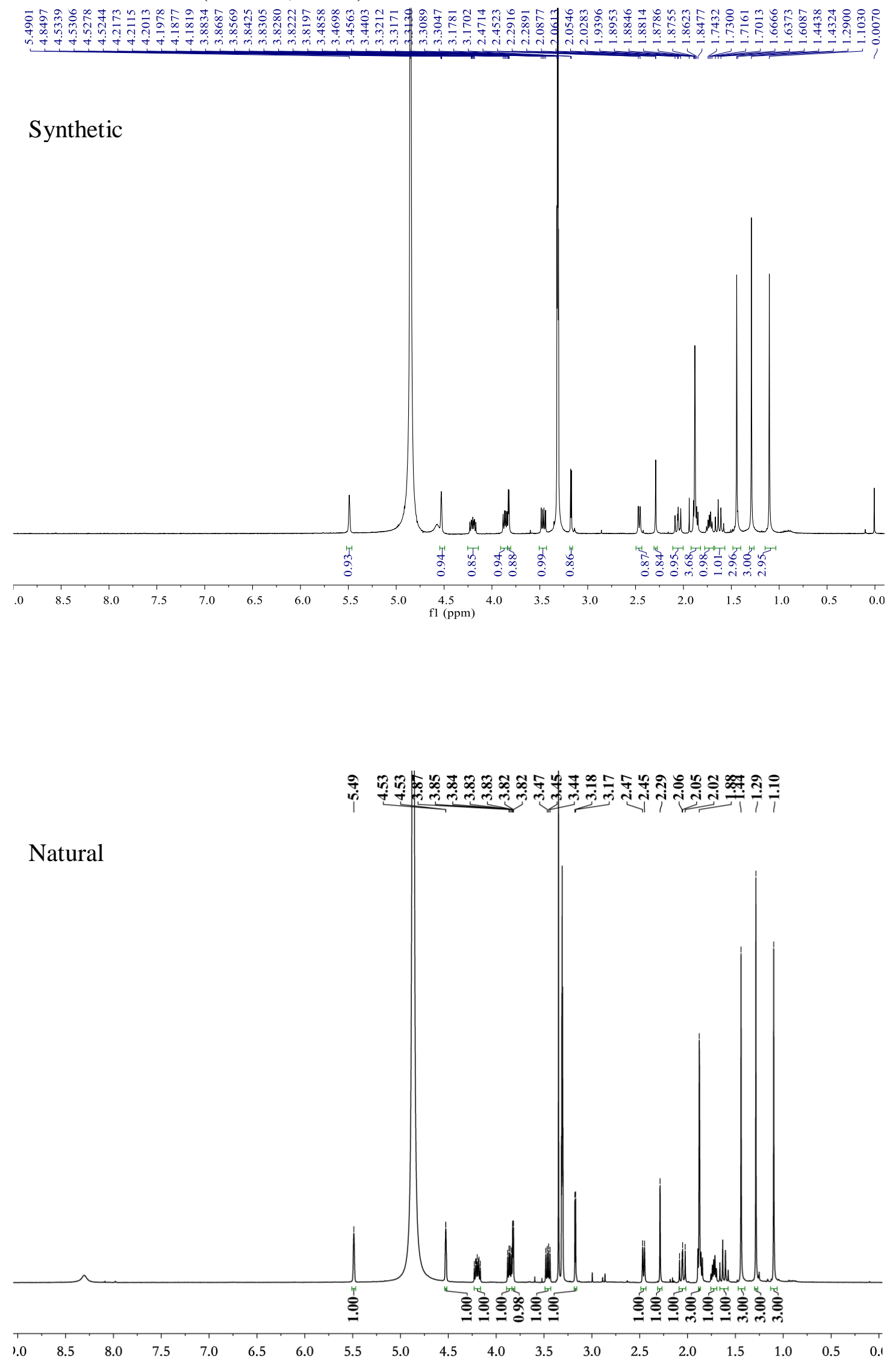
Figure S2. Comparison of ${ }^{13} \mathrm{C}$ NMR Spectra of Synthetic and Natural (-)Rhodomollanol A $\left(\mathrm{CD}_{3} \mathrm{OD}, 25^{\circ} \mathrm{C}\right)$

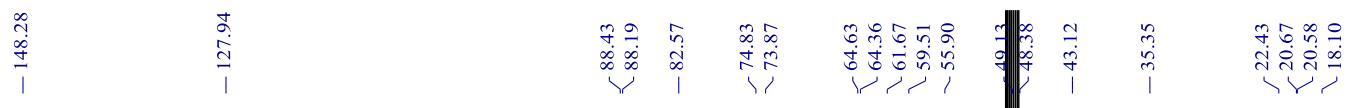

Synthetic

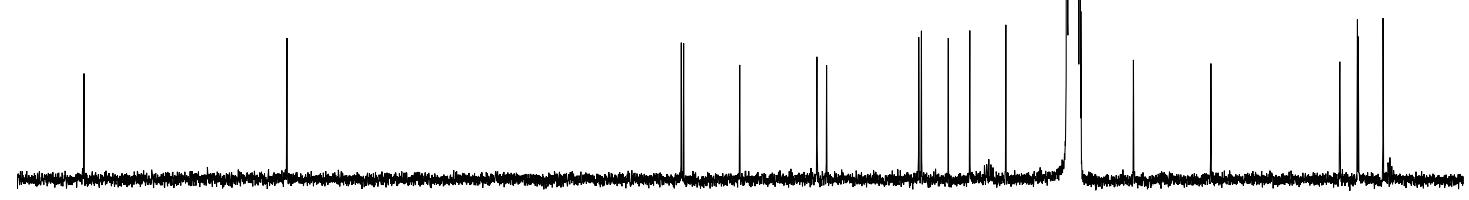

\begin{tabular}{rrrrrrrrrrrrrrrrrrrrrrrrrrrrrrrrrrr}
\hline 55 & 150 & 145 & 140 & 135 & 130 & 125 & 120 & 115 & 110 & 105 & 100 & 95 & 90 & 85 & 80 & 75 & 70 & 65 & 60 & 55 & 50 & 45 & 40 & 35 & 30 & 25 & 20 & 15 & 1
\end{tabular}

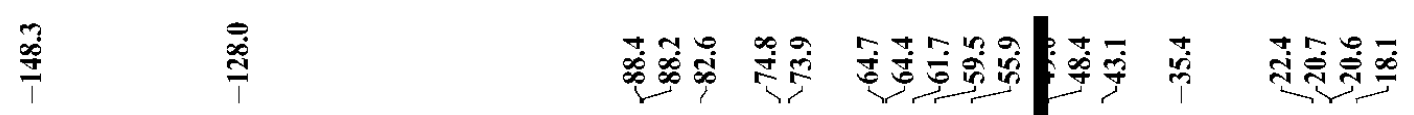

Natural

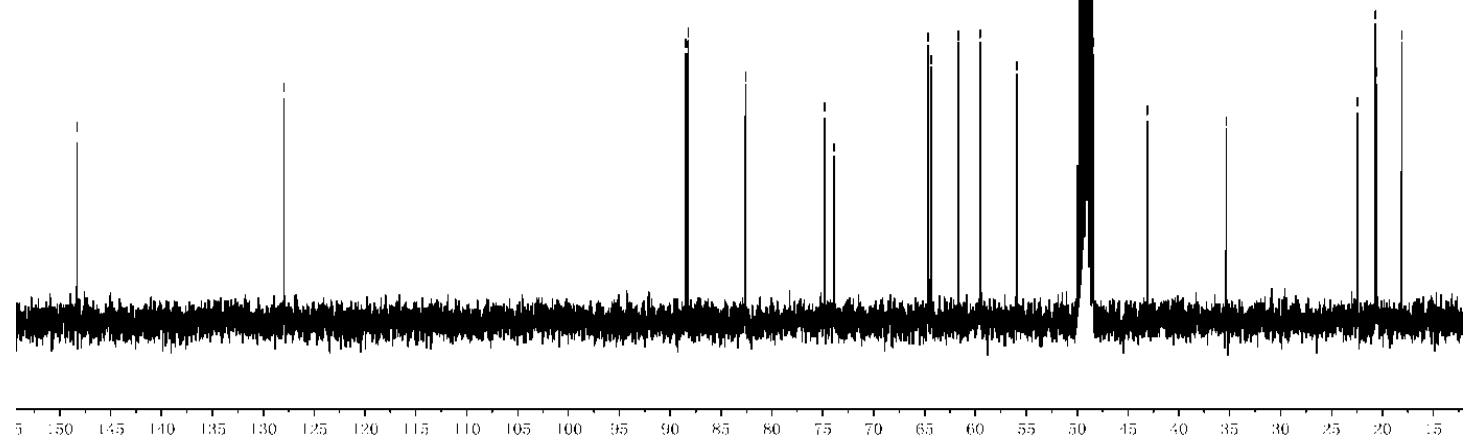




\section{V) HPLC Chromatographs}

Daicel Chiralcel OD-H $(0.46 \mathrm{~cm} \times 25 \mathrm{~cm}), n$-hexane/2-propanol $=97 / 3, \mathrm{v}=1.0$ $\mathrm{mL} \cdot \min -1, \lambda=210 \mathrm{~nm}$.

$\mathrm{mV}$

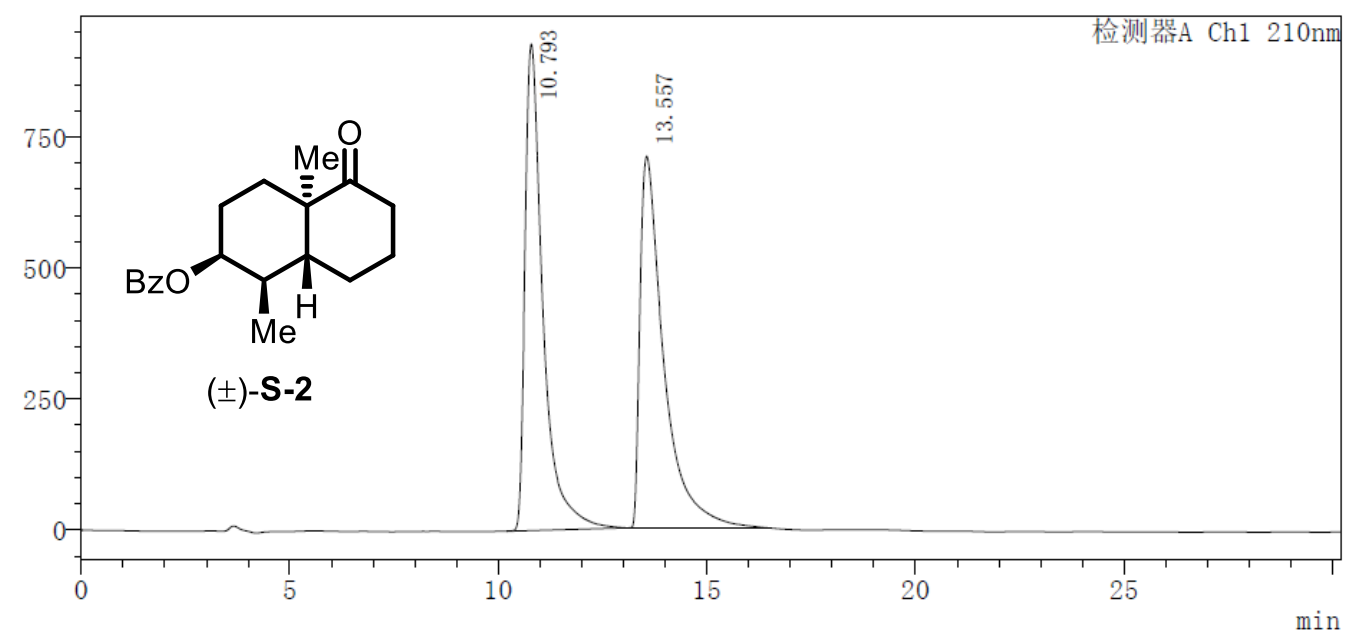

检测器A Ch1 $210 \mathrm{~nm}$
\begin{tabular}{|r|r|r|r|r|r|r|r|}
\hline 峰号 & 保留时间 & 面积 & \multicolumn{1}{c|}{ 高度 } & 浓度 & 浓度单位 & 标记 & 化合物名 \\
\hline 1 & 10.793 & 27848972 & 928332 & 49.793 & & $\mathrm{M}$ & \\
\hline 2 & 13.557 & 28080034 & 708858 & 50.207 & & $\mathrm{M}$ & \\
\hline 总计 & & 55929006 & 1637189 & & & & \\
\hline
\end{tabular}

$\mathrm{mV}$

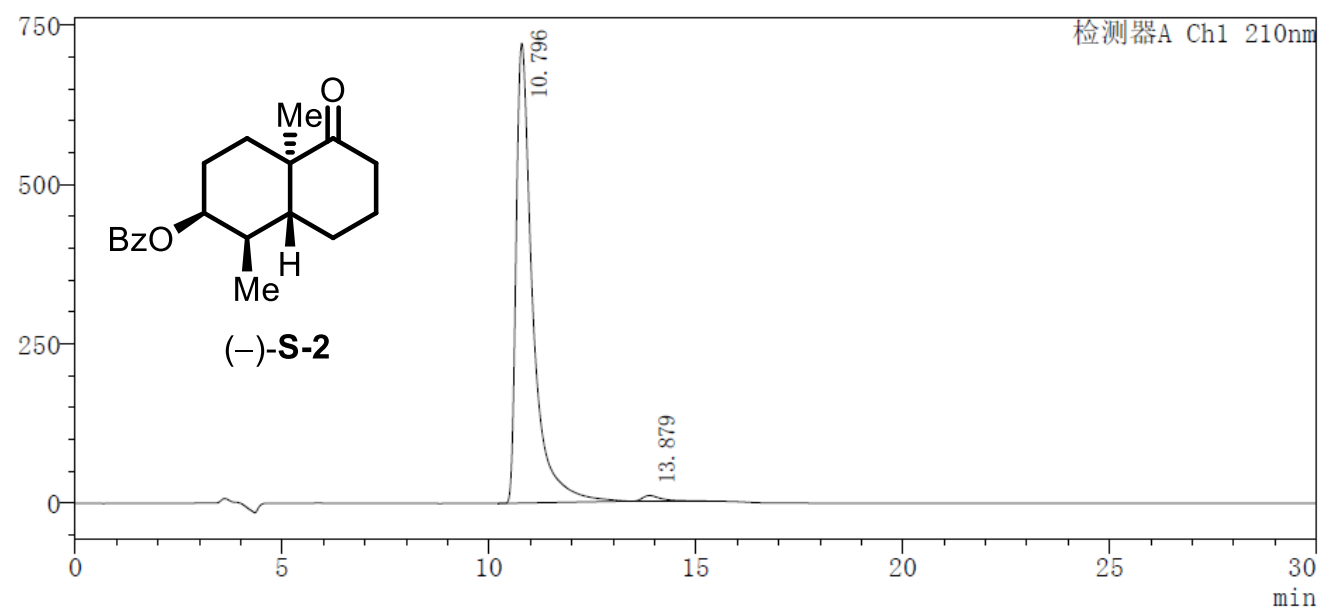

检测器A Ch1 $210 \mathrm{~nm}$

\begin{tabular}{|r|r|r|r|r|r|r|r|}
\hline 峰号 & 保留时间 & \multicolumn{1}{|c|}{ 面积 } & \multicolumn{1}{|c|}{ 高度 } & \multicolumn{1}{|c|}{ 浓度 } & 浓度单位 & 标记 & 化合物名 \\
\hline 1 & 10.796 & 20134236 & 721025 & 98.543 & & $\mathrm{M}$ & \\
\hline 2 & 13.879 & 297625 & 8764 & 1.457 & & $\mathrm{M}$ & \\
\hline 总计 & & 20431862 & 729789 & & & &
\end{tabular}

